\title{
DIGITAL ACTION CAMPAIGNS IN THE GLOBAL PUBLIC SPHERE
}

\author{
BY \\ JUMOKE GIWA
}

\begin{abstract}
A thesis
submitted to the Victoria University of Wellington

in fulfilment of the requirements for the degree of

Doctor of Philosophy in Media Studies
\end{abstract}

Victoria University of Wellington

(C) 2017 
We're here. And then we're not. Got it!

$-\mathrm{jg}$ 


\section{Abstract}

This research project undertakes a critical analysis of the use of new media technologies by community activists engaging in local and global communities. Increasingly, community organizations are using digital media to augment their various activities and conduct campaigns. I will consider this development with regard to WorldPulse.com, a global organization whose aim is to foster and facilitate civic engagement. More specifically, the website attempts to function and serve as a global public sphere and vehicle for the expression and discussion of political, social and cultural issues relevant to women. The analysis conducted in this thesis focuses on the website's digital action campaigns on gender-based violence, girl child education, and women's access to technology between 2012 and 2014, and its 'Voices of Our Future' citizen journalism training program.

This project employs digital ethnographic methods using content and discourse analysis, participant observation, online web survey, semi-structured email interviews and a researcher's journal to examine the potential of worldpulse.com to serve as a global public sphere for women. The research makes use of critical studies theories and data triangulation methodologies in order to identify and evaluate if, and to what extent, the site facilitates public sphere activity and activism. I have developed an inductive typology to assess levels and kinds of civic engagement that is enabled and augmented by the interconnection of online and offline advocacy. This thesis aims to contribute to the body of scholarly literature researching and evaluating the extent to which new media technologies enable and facilitate public sphere engagement. 
Dedication

I dedicate this thesis to my Giwa clan: to every single member. 


\section{Acknowledgements}

I would like to thank my family and friends for their love and support while I was working on this thesis. My very special and heartfelt gratitude goes to the Giwa Gals Oluwatidawape and Titoluwanimi. Thank you SNM for the additional and rare gift of presence.

I am especially grateful for the constructive critique, valuable feedback, wisdom, helpful guidance, formative nudging, and re-assuring support of my thesis supervisor, professor Tony Schirato, who has invested so much time into my graduate studies. You made me work harder, think better, reach further, and write much better. Your unwavering guidance and support were crucial in producing this work. Thank you very much, Tony.

Thank you to my doctoral thesis examiners for their constructive critique and valuable feedback that further enriched this thesis.

Very special thanks to the Social Sciences and Humanities Research Council of Canada for the SSHRCC doctoral fellowship and Victoria University of Wellington for the Victoria doctoral scholarship and Victoria submission scholarship.

My heartfelt gratitude goes to the WorldPulse.com community members who produced the archived journals on the platform, as well as those who invested additional time in this project by completing the online web survey and email interviews. Additional thanks goes 
to World Pulse staff for participating in the email interviews for the purpose of conducting this research.

I also thank my sister and friend, Donna Pearce, for her proofreading assistance. I am also grateful to the members of the School of English, Film, Theatre and Media Studies team, including faculty and staff, for their assistance. Specifically, I am grateful to associate professor Trisha Dunleavy for your guidance and support over the course of my studies.

There's something about this land. Aotearoa. 


\section{Contents}

Abstract iii

Dedication $\quad$ iv

Acknowledgements $\quad$ V

$\begin{array}{ll}\text { Introduction } & 1\end{array}$

Parameters of the Thesis Topic...........................................2

Significance of the Thesis Topic............................................ 3

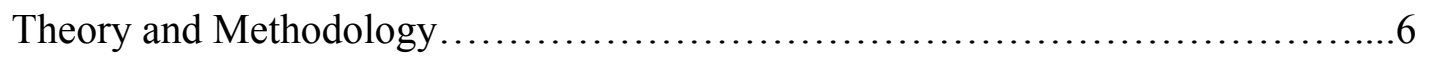

Chapter Plan................................................................9

$\begin{array}{lr}\text { Chapter One: Theoretical Framework } & 12\end{array}$

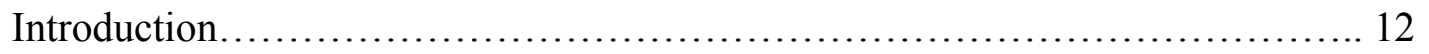

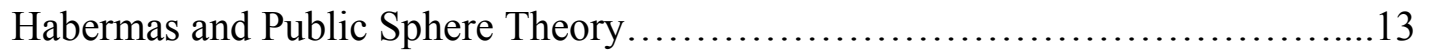

Habermas and Communicative Action Theory............................. 18

The Public Sphere: Class, Discourse and Power.............................22

The Public Sphere and Intellectuals.......................................

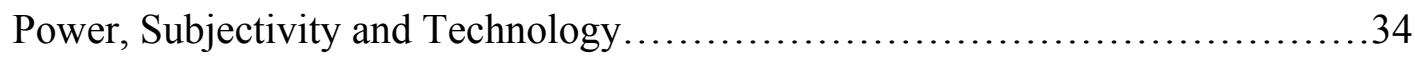

Reflexivity and Knowledge........................................... 38

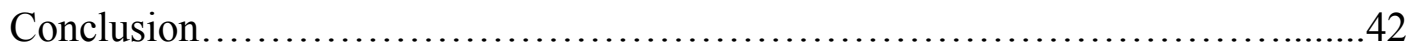

Chapter Two: Literature Review 446 


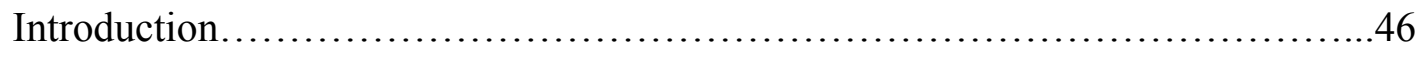

The Public Sphere and Communicative Action....................................46

New Media and the Public Sphere...........................................52

Gender and Public Sphere Activity...........................................61

Social Movements and the Public Sphere...................................67

The Global Public Sphere................................................. 72

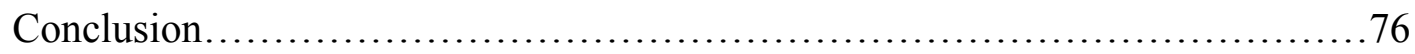

Chapter Three: Methodology

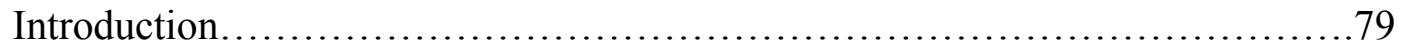

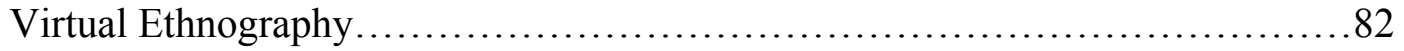

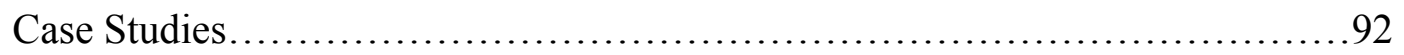

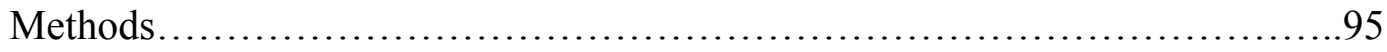

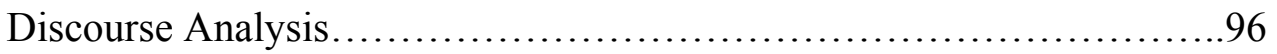

Reflexivity and Critique.....................................101

Content Analysis and the Digital Action Campaigns.....................105

Online Web Survey: the 'Voices of Our Future' Citizen Journalism

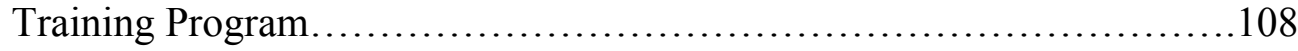

Semi-Structured Interviews: WorldPulse.com Participants and

Management.......................................................... 114

Research Design........................................................ 119

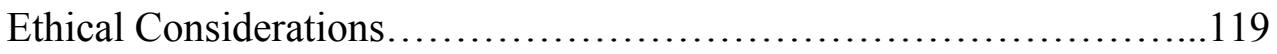

Researcher's Journal............................................... 120 
Journaling on WorldPulse.com..........................122

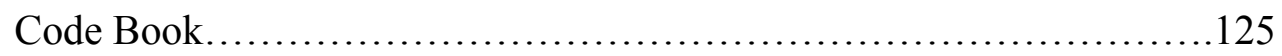

Conclusion........................................................ 126

Chapter Four: The Internet and WorldPulse.com 129

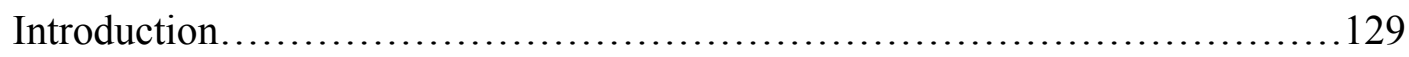

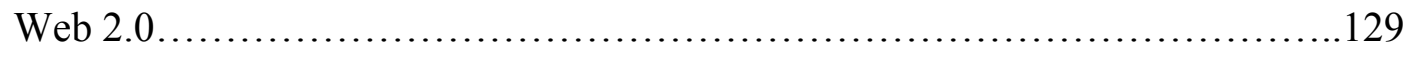

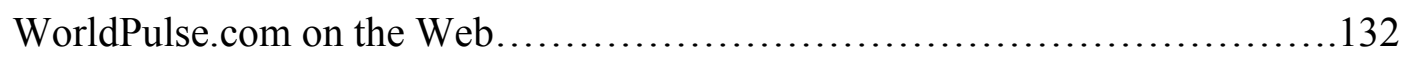

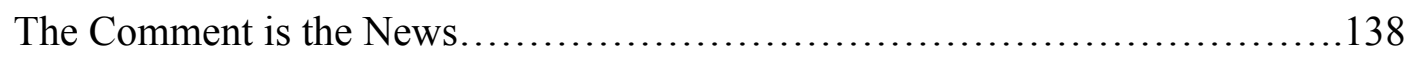

The 'Voices of Our Future' Citizen Journalism Training Program...............140

Conclusion........................................................ 145

Chapter Five: Overview of the Research Data 148

Introduction ....................................................... 148

Content Analysis of the Digital Action Campaigns........................... 149

Ending Gender-Based Violence 2012 (EGBV)......................152

Girls Transform the World 2013 (GTTW)........................ 155

Women Weave the Web 2014 (WWTW)............................. 157

Online Web Survey of the WorldPulse.com Community.................159

'Voices of Our Future' Citizen Journalism Training Program.............159

Computer-Assisted Software: NVivo and SPSS.......................... 168

Online Web Survey Report........................................... 170

Email Interviews with WorldPulse.com Community Members and 
World Pulse Staff.................................................. 178

Technology and Autonomy.....................................180

Skill and Social Support........................................181

Digital Action Campaigns........................................ 184

'Voices of Our Future' Citizen Journalism Training Program.............188

Researcher's Journal................................................. 190

Conclusion.......................................................... 192

Chapter Six: Public Sphere Activity and Engagement on WorldPulse.com 195

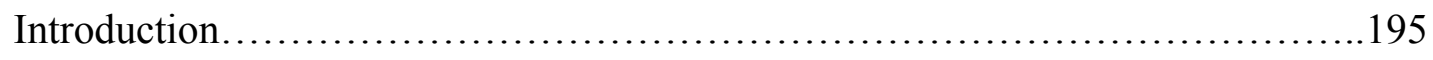

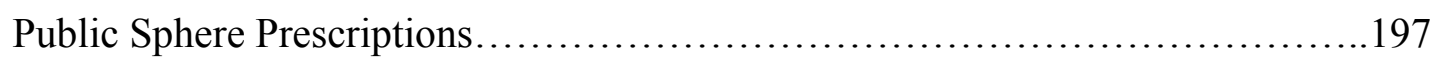

Individual Agency and Independence of Site of Discourse................198

Rationality and Emotionality..................................202

Reflexive Engagement.........................................212

Empathetic Discourse and Engagement............................220

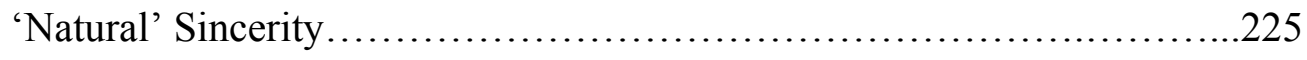

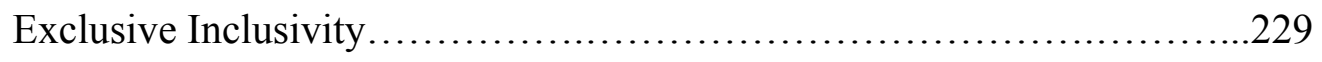

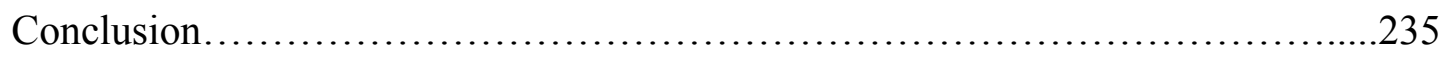

\section{Chapter Seven: Digital Inequality, Public Sphere Activity, and}

$\begin{array}{lr}\text { WorldPulse.com } & 239\end{array}$

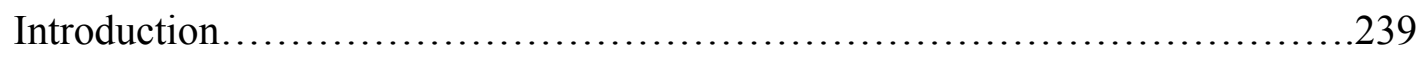

Five Broad Forms of Inequality ..................................... 240 


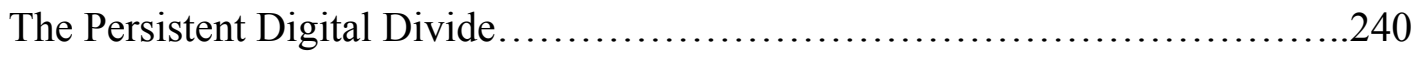

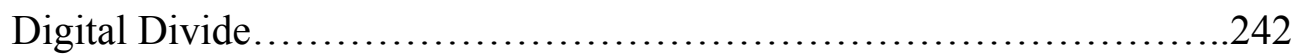

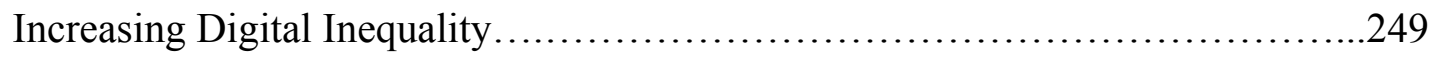

Digital Inequality - Individual and Groups...........................252

Digital Inequality - Institutional..................................254

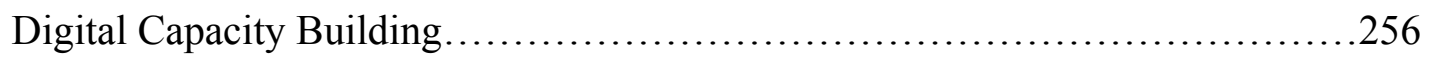

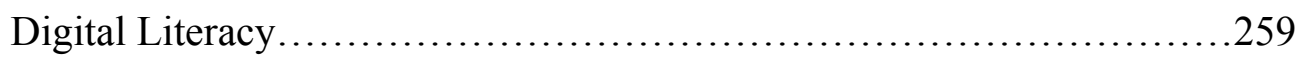

Individual Capacity Building........................................264

Team Based Capacity Building......................................267

Capacity Building - Institutional...................................270

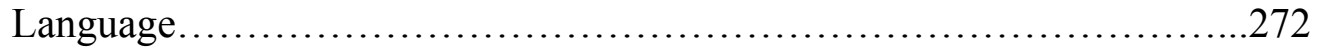

Socio-Cultural Inclusivity.................................................276

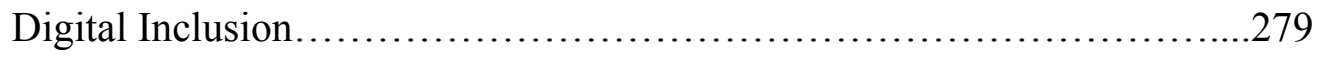

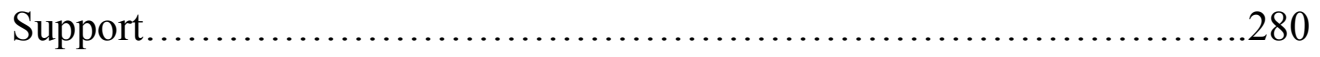

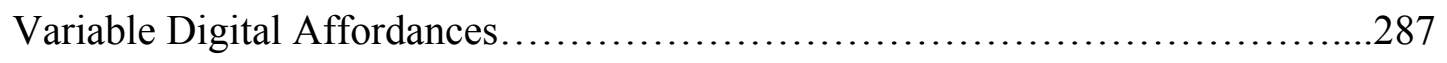

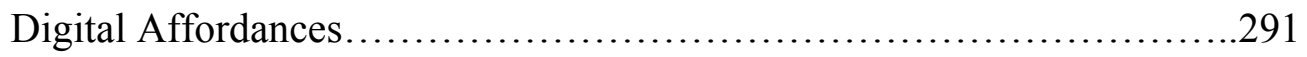

Digital Empowerment - Individual or Group...........................294

Digital Empowerment - Institutional................................296

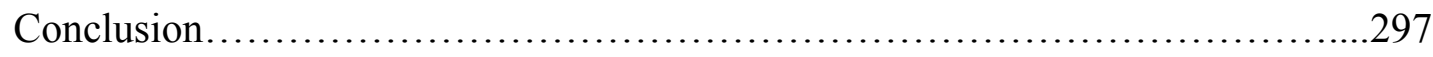

\section{Chapter Eight: Civic Engagement Typology and the Digital Action}

Campaigns on WorldPulse.com $\quad 300$

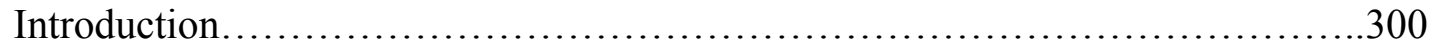


Demographics. 301

Issues: The Comment is the News..............................................304

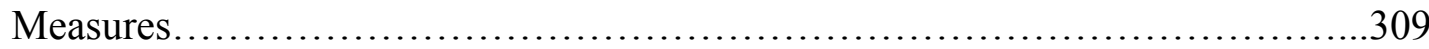

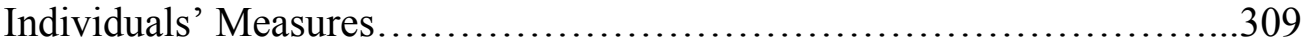

Institutional Measures................................................. 311

Measurable Actions.........................................................

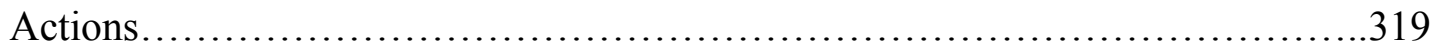

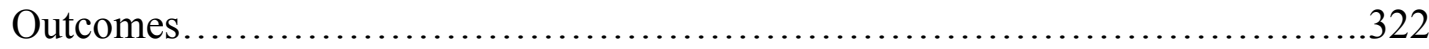

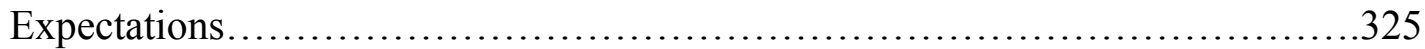

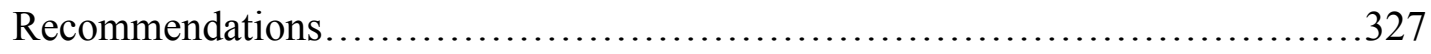

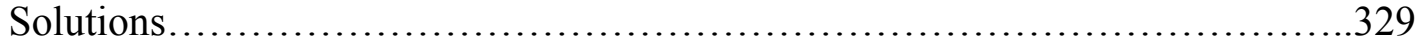

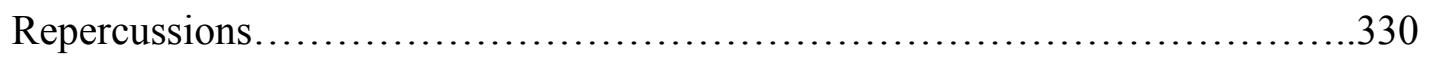

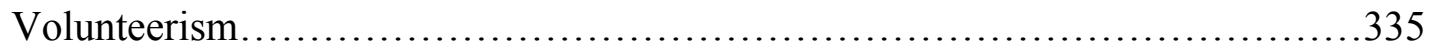

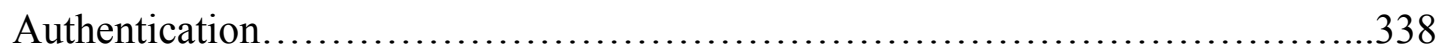

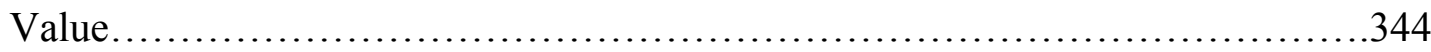

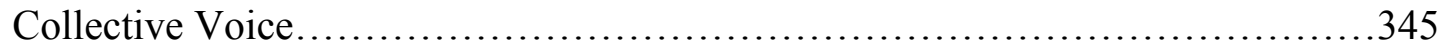

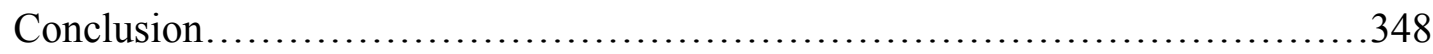

Chapter Nine: An Analysis of the Themes Identified in the Data from

$\begin{array}{ll}\text { WorldPulse.com } & 352\end{array}$

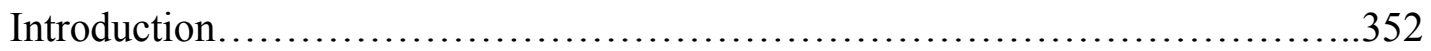

WorldPulse.com as a Micro Public Sphere......................................353

Agency, Rationality and Emotionality, and Exclusive Inclusivity............355 
Reflexive Engagement, Empathetic Discourse, and 'Natural' Sincerity.....358

The Digital Divide and Digital Inequality.................................. 360

The Persistent Digital Divide, Capacity Building, and Digital Affordances.362

Digital Inequality and Socio-Cultural Inclusivity......................363

Embedded e-Activists: The Intersection of Online and Offline Advocacy...........365

WorldPulse.com and Political Action..................................367

Demographics, Authentication, and Volunteerism.......................371

Issues, Actions, Measures, and Outcomes.......................... 372

Expectations, Recommendations, Solutions, and Repercussions..........373

Value and Collective Voice........................................375

WorldPulse.com as a Global Community ................................ 375

WorldPulse.com as a Supportive Space.............................376

WorldPulse.com Giving Women a Global and Collective Voice...........379

WorldPulse.com as an Inclusive Platform...............................381

WorldPulse.com as an Accessible Platform..........................382

WorldPulse.com Influencing Policies in Local and Global Communities..382

WorldPulse.com, Identity and the Digitalized Public Sphere....................383

Participation in the Digital Public Sphere...................................385

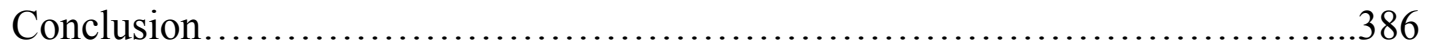

$\begin{array}{ll}\text { Conclusion } & \mathbf{3 9 0}\end{array}$

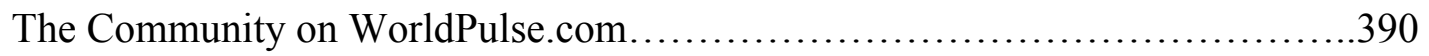

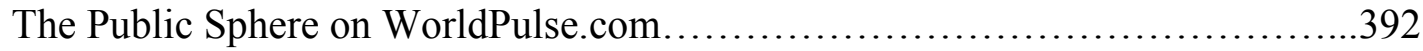




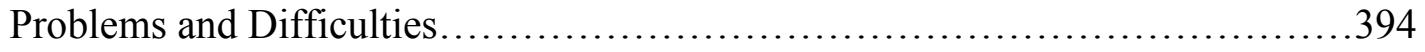

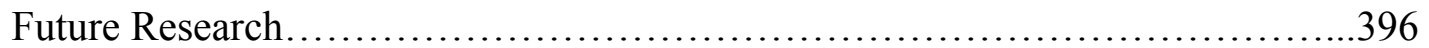

$\begin{array}{ll}\text { Bibliography } & 398\end{array}$

$\begin{array}{ll}\text { Appendix } & 451\end{array}$

Abbreviations....................................................................451

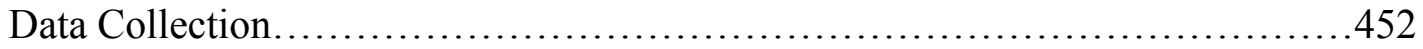

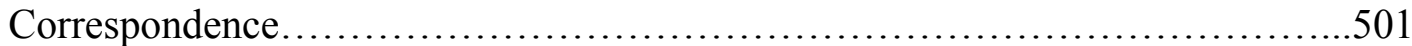

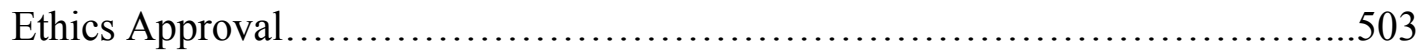

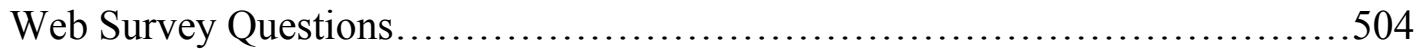

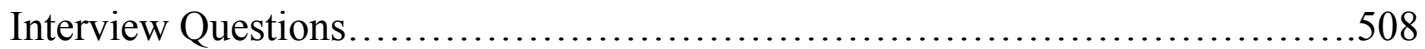

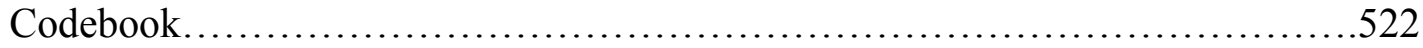


Introduction

The theoretical and scholarly context and departure point of this thesis is the proposition, widely discussed, debated and analyzed in academic literature, that digital technology, and the Internet in particular, has both effected a transformation of the notion of what constitutes the public sphere, and in the process rendered earlier formulations of how the public sphere functions (or theoretically functions), more or less anachronistic and irrelevant, or at the very least in need of serious reformulation. In order to consider, address and test this proposition, this thesis will analyze how, and the extent to which, WorldPulse.com (WPc), an independent, non-profit, non-governmental media organization, functions as a global public sphere site, most particularly for the discussion of political, social and cultural issues relevant to women. I will describe, analyze and evaluate the extent to which WPc facilitates public sphere contributions and exchanges among and across people and groups whose voices are often marginalized in mainstream media reporting.

WorldPulse.com characterizes and promotes itself as a public sphere site and space for marginalized groups, especially women. An assessment of the claims that the site makes about its public sphere functions and status is informed by the following research questions:

- To what extent does WorldPulse.com function as, and constitute, a site or space of public sphere activity? 
- To what extent do activities on WorldPulse.com translate into social, cultural or political actions, such as changes in policy and mode of governance in the members' communities?

- How can we characterize and organize the different contributions of participants on WorldPulse.com?

- To what extent does the move to a virtual or digital public sphere require a reformulation of the notions of identity and identification, which is a critical issue for analyzing and understanding the roles, functions and parameters of the public sphere?

Parameters of the Thesis Topic

The Internet and new media technology have created opportunities for more people to engage in political processes, although many scholars in the field of new media studies warn that one should avoid the pitfalls of using utopian and dystopian characterizations in assessing the potential of the Internet as a communication tool (Blumler and Gurevitch, 2001; Dean, 2003; Moe, 2008). Other scholars contributing to this debate propose that the Internet supplements most users' already existing cache of communication tools, rather than simply replacing them (Dahlgren, 2005; Papacharissi, 2002; Wellman, Quan-Haase, Witte and Hampton, 2001). People participating in political forums on the Internet may also engage offline in the political process, and vice versa. This thesis, and the above research questions out of which it will be constituted, are part of this ongoing academic debate about, 
and research into, the relationship between digital media and contemporary public sphere activity.

This research is also concerned with issues of 'digital divide' and 'digital inequality,' which have arisen with the advent of the Internet as an important communication tool (Albrecht, 2006; Alzouma, 2013; DiMaggio and Hargittai, 2001; Janack, 2006; Raboy and Shtern, 2010). A lack of skills and literacies hinder or prevent those who already have access to the Internet from engaging with other online communities and activists. In extending this kind of research to take in the activities onWorldPulse.com, I will examine the extent to which access to the Internet facilitates or hinders women's participation in online forums, and what steps women are taking to overcome the challenges they might face in accessing those forums.

One of the aims of the project is to examine how WorldPulse.com uses its digital action campaigns to promote and facilitate public sphere activity among a diverse group of women, particularly women from areas that are not strongly represented in global public sphere debates and exchanges. Another aim is to critique the effectiveness of this platform: this second aim is tied in with an analysis of the culture of WPc, and of the extent to which members can negotiate their interactions and relationships on the platform.

Significance of the Thesis Topic 
One of the more general aims of sites such as WorldPulse.com is the globalizing of citizen involvement in issues that have communal consequences. This is in response to the position that programs promoted or sponsored by international agencies, such as the United Nations, use 'hand-picked' country delegates rather than seeking broader citizen involvement. This research looks at how WorldPulse.com attempts to foster and facilitate forms of civic engagement that go beyond conventional political and public sphere sites, spaces and genres; and perhaps equally importantly, beyond those relatively privileged groups that conventionally constitute public sphere activists. In the age of digital technology, relatively minor activities such as the forwarding of information, via retweets or by forwarding an emailed 'call for action', are taking on increasing political significance.

This research constitutes an attempt to analyze how the opportunities for political and public sphere involvement provided by digital technology can be read against the various theoretical definitions of what the public sphere is, and how it operates. Through this research, two significant and closely related research tasks are being undertaken: firstly, the claim that digital technology extends and facilitates public sphere activity is being considered and evaluated at a micro-level (that is, by focusing on WorldPulse.com); secondly, and by way of extension, the results of that analysis can be fed back into a theoretical reappraisal of the notion of the public sphere (and in particular, a reappraisal of the extremely influential version proposed by Habermas). These considerations constitute the main significance of this thesis. 
There are theoretical difficulties in undertaking this task, not least being the difficulty associated with using a concept such as Habermas' notion of the public sphere as a frame with which to gather information that can then be used to critique the original theoretical frame. Moreover, Habermas' public sphere theory presumes that interlocutors will communicate about, and discuss and attempt to negotiate, issues of common interest. We can say that for Habermas, public sphere activity is first and foremost a question of communication. It is quite apparent, however, that Habermas' understanding of the contexts, mechanisms, rules and pragmatics of communication are relatively undeveloped, particularly in terms of what we can call the cultural politics of discourse. Accordingly if we are to provide a critique of Habermasian public sphere theory, we need to consider more than just developments in technology; we also have to take into account the extent to which all forms of communication are bound up with, and informed by, the cultural politics of communication.

Foucault's work, and in particular his notion of discourse, is relevant and useful here. Foucault proposes that discourses are not just a collection of signs, but "practices that systematically form the objects of which they speak" (Foucault, 1972, p. 49). By this, he means that discourses are not a reflection of something that is already there, or a representation of existing meaning and form; rather, they determine how we experience, order and evaluate the world. Moreover, since discourse is largely authorized by and through the workings of power, then every discursive utterance or act carries the trace of, and in a sense reproduces, a worldview or set of meanings commensurate with, informed by 
or derived from power. To be able to name, categorize or label someone or something is to exert power and to produce material effects.

These theoretical difficulties, while not inconsiderable, only emphasize and underline the value and significance of this research and its aims. Habermas' theories continue to exercise an influence over how the public sphere is understood, despite being the subject of critiques from scholars who argue that they are technologically outdated, and that they fail to incorporate the cultural politics that contextualize all communicative action. This research will attempt to consider both these sets of issues; this will constitute the contextual and theoretical criteria within which the relationship between Habermasian theory and contemporary public sphere activities is evaluated.

Theory and Methodology

I use theoretical triangulation in this study by drawing on the works of Habermas, Foucault and other relevant theorists, most particularly those who critique the Habermasian notion of the public sphere. I utilize Habermas' theories as a frame for, and as a means of, identifying and contextualizing evidence as to how the activities on WorldPulse.com can be evaluated. It may be that such activities are not commensurate with the criteria laid down by Habermas; however, it could be argued that sites such as WorldPulse.com can be read as constituting digital forms of the Habermasian public sphere. Another possibility, however, is that Habermasian theory can be used to evaluate the extent to which communicative action on WorldPulse.com at the very least aspires to, or understands itself within, the field 
outlined by that criteria, and that the extension of this digitally-based public sphere activity can be used to problematize and extend beyond the limitations of Habermasian theory.

The methodology can be characterized as a virtual ethnography that utilizes a mixed methods case studies approach. I conducted the case study using theoretical and data triangulation so as to explore multiple dimensions of the research questions. This thesis is effectively a virtual ethnographic study of WorldPulse.com. I conducted a multi-stage data triangulation of information, which was largely obtained through content analysis and a web survey. The discourse and content analysis as well as the web survey will be augmented by follow-up semi-structured email interviews and a researcher's journal. One of the main entries in my researcher's journal is about my first-hand experience as an embedded e-activist on the site. Moreover, my experiences participating in the \#bringbackourgirls campaign were helpful in assessing the various levels at which these groups of citizen journalists are involved in civic engagement.

The discourse and content analyses assess the archived journals on WPc. I administered an online web survey that was particularly useful for helping to understand why members use, and what kind of work they do on, the WPc forum. The third phase of data collection consisted of semi-structured email interviews that enabled me to better assess and contextualize the information that was obtained from the archived journals and online web survey. The interview responses presented and analyzed in chapters five to eight are cited using NR (No Reference). This mixed methods approach helped to produce a detailed account of the activities, discourses and strategies that are used on the WPc website. 
I used multiple theories drawn from critical theory, sociology, gender studies, communication studies and more generally from the cultural studies paradigm, in order to conduct a critical evaluation of the data. In conducting this evaluation using multiple frameworks, I was able to assess, for instance, questions such as whether or not WPc enables or constrains the representation of a diversity of women's voices on its forum. Triangulation is a multi-prong approach to the assessment of a social phenomenon; the data triangulation uses content analysis, self-administered online web survey questionnaire, semi-structured email interviews, and a researcher's journal to collect data from WPc members and management.

In doing research of this kind, there are always issues about the validity of the information that is produced, and the methodologies that facilitate its production. Lockyer (2006) suggests that "many authors have rejected the assumption that convergence of results from different research approaches can be interpreted as a sign of validity" (p. 42). She also notes that "although there may be strong correlations between the results of differing tests, the possibility of error is maintained, as both research approaches could be problematic" (p. 42). I addressed these issues through careful and rigorous use of sequential triangulation; the results from the content analysis informed the focus of the web survey. These results were also a useful guide in developing questions for the semi-structured interviews.

I also used computer-assisted software (NVivo and SPSS) for coding and analyzing data, and for developing the civic engagement typology for embedded e-activists. The categories 
of analyses were derived from the six criteria of the Habermasian public sphere, the five broad forms of inequality by DiMaggio and Hargittai, and the civic engagement typology for embedded e-activists.

\section{Chapter Plan}

The first three chapters are predominantly concerned with outlining the theoretical and methodological frames that inform this thesis. Chapter One will introduce and critique Habermas' theories of the public sphere and communicative action by way of the work of Foucault, Marx, Bourdieu and feminist theory. The chapter will discuss the ways that the public sphere and communicative action have been envisioned and how they have functioned for various communities. Chapter Two will introduce and discuss existing scholarly literature about the public sphere in relation to online spaces; citizen engagement with democratic processes in various countries; the use of social media for engaging in public sphere activity; how social activists are combining old and new media for engaging in communities online and offline; and the growing debate about transnational and global publics facilitated by new media technologies. Chapter Three will focus on the methodology employed in this thesis. The study is a virtual ethnography that employs case study methodology. The case study includes dialectical theory and data triangulation that uses discourse and content analysis of archived journals, online web survey of the WPc community, semi-structured email interviews with WPc community members and staff, and a researcher's journal. The chapter will also discuss my volunteer work as a 'Voices of Our Future' listener and 'Digital Action Campaign' commentator and assessor while I was a 
participant-observer on the website.

The remaining chapters will discuss the WPc website and technology, and present, analyze and evaluate the research data. Chapter Four will provide a brief overview of Web 2.0 with specific regard to how WPc sets itself up and functions and how it produces itself and its work discursively. Chapter Five will provide a detailed report of the three sets of data obtained from the archived journals of the digital action campaigns on WorldPulse.com, online web survey questionnaire and semi-structured email interviews, plus material from the researcher's journal that I will keep throughout this project. The data sets will be used to conduct an extended critical assessment of the abilities and capacities of WorldPulse.com community members in their use of technological access, technical knowledge and language skills. Chapters Six, Seven and Eight will undertake an analysis of the data produced in the research, using the analytical frameworks of Habermas (Chapter Six), DiMaggio and Hargittai (Chapter Seven), and the civic engagement typology that I developed while conducting this research (Chapter Eight). Habermas' public sphere theory has been operationalized by Dahlberg so as to provide a methodological model for assessing public sphere sites and practices. The five broad forms of inequality identified by DiMaggio and Hargittai provide categories of analysis that are useful for assessing the engagements and practices of WPc community members in relation to their use of digital technology for civic engagement.

In Chapter Nine, I will provide an analysis of the discursive themes emerging from all the data. In the first section, I discuss the ability of WPc to serve as a global public sphere site; 
the second section of this chapter discusses the five broad forms of inequality identified by DiMaggio and Hargittai, and situate them within the context of more recent data; section three provides a description and assessment of the work of embedded e-activists; finally, section four evaluates WPc culture and practices in terms of its stated aims and imperatives. I will conclude this dissertation by summarizing the results and limitations of my research, and identifying directions for further study. 
Chapter One: Theoretical Framework

Introduction

The primary body of theory to be used in this research project is derived from Habermas' (1974) work regarding the public sphere and the revisions of this theory that he makes in his later work on communicative action (1981). I also draw on the works of various critical theorists, including Marx (1978), Foucault (1972), and Bourdieu (2000). This chapter will provide a critical and evaluative account of the diverse scholarships from the social sciences and humanities regarding the public sphere. Specifically I examine public sphere critique in relation to gender; the intersection of discourse, truth and power, including the functions of public and organic intellectuals in the digital domain; subjectivity and the influence of old and new media on representations in digital space; and the requirement of reflexivity as a vital part of the process of assessing civic engagement activities online.

In the first two sections of this chapter, I discuss the works of Habermas, Fraser, Pateman and Bourdieu, primarily with regard to the relation between the public sphere and gender politics. The next two sections discuss the theories of Marx, Foucault and Habermas, with specific focus on class relations and the relation between capitalist commercial media, intellectuals and public sphere activities. This is followed by a discussion of power and subjectivity and its relation to cultural production and consumption. Finally, I look at the role of reflexivity and reflective practices in assessing issues of inequality and injustice. 
Habermas and Public Sphere Theory

Habermas defines the public sphere as:

A realm of our social life in which something approaching public opinion can be formed. Access is guaranteed to all citizens. A portion of the public sphere comes into being in every conversation in which private individuals assemble to form a public body. They then behave neither like business or professional people transacting private affairs, nor like members of a constitutional order subject to the legal constraints of a state bureaucracy. Citizens behave as a public body when they confer in an unrestricted fashion - that is, with the guarantee of freedom of assembly and association and the freedom to express and publish their opinions - about matters of general interest (Habermas, 1974, p. 49).

Habermas' conceptualization of the public sphere indicates the emancipatory potential of citizens when they assemble as a public body. He identifies three different ruptures in the development of the public from representative publicity (which was largely about feudal lords representing themselves to the people) to literary publicity, and later on to the emergence of the bourgeois political public. Habermas' acknowledgement of the emancipatory potential of citizens is a departure from Lippman (1925), who conceives of the public as having no political importance or significance, and in need of experts to provide solutions to citizens' problems. The Middle Ages to the $18^{\text {th }}$ century were, for 
Habermas, characterized by representative publicity. The dawn of the $18^{\text {th }}$ century saw the appearance of the bourgeois public sphere.

The Habermasian public sphere is based on the ideal type model, a Weberian concept that prescribes characteristics required to achieve an ideal situation. The Habermasian ideal type of the public sphere requires and is predicated upon an ideal speech situation that places a strong emphasis on rationality. This requirement for rationality can be seen in the rules that he outlined in his theory of discourse ethics:

1) Every subject with the competence to speak and act is allowed to take part in a discourse; 2a) Everyone is allowed to question any assertion whatever; 2b) Everyone is allowed to introduce any assertion whatever into the discourse; 2c) Everyone is allowed to express his attitudes, desires, and needs; 3) No speaker may be prevented, by internal or external coercion, from exercising his rights as laid down in (1) and (2) (Habermas, 1990, p. 86).

These rules reiterate Habermas' commitment to discourse participants' freedom to participate and freedom from coercion, which were first introduced in his theory of the public sphere (1974). An ideal speech situation is fundamental in Habermas' ideal type of the public sphere, which is a metaphorical space of public discussion and deliberation. Deliberation would be ideally based on logical reason and undergirded by consensus.

Habermas' public sphere theory features three key elements: accessibility, discursive 
inclusivity and equality, and autonomy. In the first instance, interlocutors engaging in public sphere deliberations are presumed to have equal access to relevant sites and spaces, metaphorical and otherwise. This presumption does not take into account, however, the reality that participants' unequal positions and power relations can and do influence the mode or outcome of such engagements. Nancy Fraser, and other scholars like Tully (2013) and Dahlberg (2005), have drawn attention to this oversight, arguing that the presumption of equal access to a sphere for deliberation is a utopian ideal. Fraser suggests that issues of inequalities that are related to, or derived from, such contexts and circumstances as birth, family inheritances, social status and income disparities should be thematized, rather than bracketed or eliminated, during public sphere discussions (Fraser, 1992, p. 112).

Fraser also pointed out, through her revision of historical records, that the modern or contemporary explosion in public engagement, exemplified for instance in the movements that developed in the United States in the 1960s, including the American civil rights movement and the women's rights movement, challenged Habermas' delimiting of the public sphere to rational-critical debate. The rise of social movements has made it necessary to rethink the hitherto elitist and bourgeois characterization of the public sphere. It has also become increasingly clear that the rational-critical argument cannot be detached from socio-cultural affects that constitute a citizen's way of life.

The second aspect of Habermas' public sphere, the necessity of discursive inclusivity and equality, has also been subject to a critique from Fraser, who argues that his conceptualization of the public sphere fails to fulfill this condition. She suggests that 
because some marginalized groups, such as women and the working class, have not historically had the means to access the bourgeois public sphere, and were not visible in, or constitutive of, the original demographic on which Habermas based his notion of the public sphere, this makes Habermas' concept limited and flawed. The gender theorist Carole Pateman, like Fraser, is particularly insistent on the issue of the gendered composition of the bourgeois public sphere. In The Sexual Contract, Pateman argues that the social contracts that guide gender relations in civil societies are characterized by patriarchal underpinnings:

Men's freedom and women's subjection are created through the original contract - and the character of civil freedom cannot be understood without the missing half of the story that reveals how men's patriarchal right over women is established through contract. The social contract is a story of freedom; the sexual contract is a story of subjection. The original contract constitutes both freedom and domination. Civil freedom is not universal. Civil freedom is a masculine attribute and depends upon patriarchal right (Pateman, 1988, p. 2).

By way of extension, we can cite Pateman's observation that in addressing the continuing gender imbalance in the public life, social contract theorists have privileged the discourse of freedom at the expense of domination, thereby omitting an important part of the contract that should be reviewed to further achieve inclusivity and equality. 
The notion of autonomy is also a subject of contention for Fraser. While Habermas argues that the sphere should be independent from state and economic influences, Fraser suggests that autonomy is not a necessary or essential feature for the spaces used in facilitating public sphere discussions. People with no affiliation to a formal group or political position, but who may temporarily identify with a position, can be significant contributors to public sphere activity. However, these 'of the moment' communities are never outside the world or the operations of power: they are always inflected by, interpellated through, and largely constituted out of the discourses, narratives and meanings produced in, and provided by, influential socio-cultural institutions such as the commercial media. Public sphere theory presumes that interlocutors will communicate about, and discuss and attempt to negotiate, issues of common interest. The theory presumes that interlocutors would have identified such issues prior to the commencement of communication. Fraser finds this prior identification of issues problematic, arguing instead that it is more expedient for those engaging in communication and negotiation to debate what should constitute the agenda, as opposed to having it pre-determined or presumed.

A key concern for Habermas in his conceptualization of the public sphere is what he terms refeudalization, suggesting that civil society is being co-opted by private interest groups with non-public economic interests. These groups are seen to be representing themselves to the public (in the same way that feudal lords did back in 1800s): hence they are not assembling in the interest of public good but rather are using civil society groups to benefit their own private interests and by extension, strengthen the unchecked power of the state and capitalist economic interests. He warns that politicians, private interest organizations 
and the state (mainly through public relations activities) are now using 'systems' media to represent themselves in spaces that 'lifeworld' media hitherto used for citizens' gathering and for the benefit of the public through civil society.

Habermas has since revised his position on the criterion that requires prior identification of issues prior to the commencement of deliberation. In communicative action theory (1981) Habermas acknowledges and recognizes that speech act that interlocutors engage in are underpinned by ongoing contestation and reconsideration of the issues, and hence consensus need not be achieved prior to commencement of such discussions in the public sphere. By extension, this revision also opens up the space for the consideration of the public sphere in multiple forms and an acknowledgement that there are micro sites within which public sphere deliberations initially occur at local and regional levels before the issues are fed into the larger national and or global space. For Tully:

By starting in this way, Habermas is able to acknowledge and recognize within the general public sphere the multiplicity of alternative, unofficial, professional, and specific public spheres that emerge out of the everyday exchange of views in public spaces. Yet, at the same time, he is able to argue that there is an ideal form of the public sphere immanent in yet distorted forms in the factual multiplicity (2013, p. 175)

Habermas and Communicative Action Theory 
Habermas' theory "presupposes the use of language as a medium for a kind of reaching understanding, in the course of which participants, through relating to a world, reciprocally raise validity claims that can be accepted or contested" (Habermas, 1981, p.99). Habermas posits that the different validity claims that discourse participants present in the process of deliberation can be resolved through communicative rationality. The revisiting of rationality in the theory of communicative action signals Habermas' ongoing and firm commitment to the requirement of rationality (which stands in opposition to emotionality) that he first introduced in public sphere theory. His expectation that citizens will engage in rational-critical discourse in the public sphere is perhaps one of the most significant requirements that continues to attract critique based on which his public sphere ideal is considered utopian.

The concept of communicative action presupposes rationality (based on language) as an essential ingredient for effective public action with which citizens engage. Habermas argues that communication is an essential requirement for any public action to be effective. Through this theory, he introduced the concepts of 'lifeworld' (which he identifies using $\mathrm{I}=$ influence and $\mathrm{L}=$ value commitments) and 'systems' (identified as $\mathrm{A}=$ Adaptation and $\mathrm{G}=\mathrm{Goal}$ ) (Habermas, 1981). 'Lifeworld' media are qualitative and 'lifeworld' processes occur between people while 'systems' media are quantitative and they can be represented by money and votes that are subject to manipulation by powerful political interests. Habermas argues that 'lifeworld' (individuals' values, social interactions, cultures, intercultural relations, family relations) gives legitimacy to 'systems' (the official economy, political parties, political state). In revisiting refeudalization as part of communicative 
action theory, Habermas re-asserts that critical engagement is in jeopardy because 'lifeworld' is limited by 'systems'. In this context, modernity remains an unfinished project because 'systems' media continue to colonize 'lifeworld' media. Habermas argues that citizens' ability to be rational or critical is limited by politicians' and organizations' money and power, which are being used to manufacture consent while presenting pre-selected and limited options under the illusion of choice. For the project of modernity to be completed, 'lifeworld' has to be decolonized from 'systems'; otherwise feudalism continues even when society is presumed to be modernized.

Through communicative action theory, Habermas shows that the normative public sphere he initially envisioned is now considered as a central public sphere that organizes and addresses issues that alternative public spheres cannot deal with. Deliberations in peripheral public spheres feed into this central public sphere. The central public sphere serves the function of problematizing issues and it oversees how these issues are addressed. "The liberal public sphere is designed to channel and reformulate this strange multiplicity of citizen-generated public opinions through the legally prescribed channels and procedures of communicative power so they are not excluded or disregarded, but, rather, are included and have the opportunity to influence voters and governments" (Tully, 2013, p. 177). Habermas' revisions of the normative public sphere positions it to function as a central organizing point for alternative and peripheral multiple sets of public spheres so their deliberations can be featured on a larger platform for more citizens to engage. Such is the positioning of WPc as a civil society public sphere for discussions at micro levels following which such 
discussions can then be fed into larger, global platforms like the United Nations or G8 conferences.

The appeal and relevance of public sphere and communicative action theories to my research resides in Habermas' acknowledgement of the emancipatory power of citizens' gathering and civil society. He conceptualizes that citizens have capacity to effect change, to challenge the status quo and that citizens' collective intelligence can indeed effect change in policies and governance structures. This allowance for citizens' agency stands in contrast to the pessimistic and disempowering conceptualization of the public by Lippman and The Frankfurt School scholars (Adorno and Horkheimer) who conceived of the public as a phantom and consider people generally to be gullible, inactive subjects who are easily manipulated by the media. Habermas' concepts resonate with me as someone who grew up in Nigeria and is aware of the restriction on free speech, especially during the successive military regimes when there were grave consequences for challenging authority or demanding accountability from government officials. I have observed in WPc participants' journal writings and comments similar accounts of people recounting their experiences of restrictions and limitations on freedom of expression and participation in public life. These WPc members' accounts are usually accompanied with an articulation of elation at the opportunity digital media, via online forum interaction, affords them to speak freely and engage in deliberations publicly about issues of common interest and public good.

The issues and problems that we have identified and discussed, to this point, are essentially predicated on, or derived from, Habermas' abstraction of the public sphere: for Habermas 
the public sphere can be understood as a metaphorical arena of public discussion and deliberation. Fraser critiques and expands on Habermas' ideal public sphere by applying the concept to an actual liberal-democratic state, where communication practices are always subject to, and inflected by, a certain pragmatics. Despite the limitations identified by Fraser, however, Habermas' public sphere theory remains a useful mechanism for thinking through and assessing citizens' engagement with the political processes and modes of governance (Dahlgren, 2012; Emden and Midgley, 2013; Lunt and Livingstone, 2013; Tully, 2013; Volkmer, 2014). To some extent, the theory can be read as analogous, and as having a similar discursive status, to the 'Gricean co-operative principle', which posits a set of ideals against which any field and its activities can be measured, judged and critiqued. As Bourdieu explains, while the Gricean principle "is constantly flouted", it functions as "a kind of implicit presupposition of all conversation ... which, though it is constantly transgressed, can be evoked at any time, as a reminder of the tacitly accepted rule or an implicit reference to what a conversation has to be in order to be a real dialogue" (Bourdieu, 2000, p.122).

The Public Sphere: Class, Discourse and Power

Marx's theories about social and class relations, and Foucault's work on discourse, surveillance and power, are useful tools for critiquing the difficulties that public sphere participants face in their attempts to gain access to equal inclusion and status in public sphere discussions and debates. Marx posits that class relations, and the deployment of class-based ideologies, determines how we think about and come to understand the world. 
Marx suggests that the ideas of the ruling classes are privileged because they own the means of production, and by extension this allows them to influence or determine the meanings and narratives that circulate in a social space: so at the most rudimentary level, the class that owns the means of producing materials on the Internet, for example, can inflect or decide what the technology should be used for.

Marx's theories about class relations are useful in understanding how and why capitalist logics and imperatives proliferate and are naturalized on the Internet: basically commercial transactions are clearly privileged over the use of Internet technology for civic purposes (Emden and Midgley, 2013; Marx and Tucker, 1978; Meinrath, Losey, and Lennett, 2013; Volkmer, 2014). By way of example, Meinrath, Losey and Lennett point out that these overriding commercial interests are visible even in the ways that Internet Service Providers (ISPs) design their Internet subscription services. The focus for ISPs is more on encouraging subscribers to participate in consumption and less on production. Other ways that these overriding commercial interests manifest include the ranking systems search engines like Google, Yahoo and Bing use for their search services. Most searches conducted on these platforms would often return results that have paid advertorials being privileged over other web pages. This privileging of commerce over civics alerts us to the need of a reevaluation of Habermas' criteria: it appears that the universal and ideal public sphere cannot be sustained - most especially not in online spaces - within this vastly commercialized space. Moreover, the naturalization of the desire for people to incessantly consume goods and services further limits the potential of the Internet as a viable civic forum. Marx's theories can also be used to interrogate the presumed deliberative feature of 
Internet forums, especially in relation to Habermas' ideals of accessibility and inclusiveness.

In addition, Marx's position that those participating in the exchange market are not doing so on a level playing field, is juxtaposed with the utopian discourse that the Internet is a tool that will erase barriers to access and equality. This position has virtually achieved a doxic status in popular (that is, predominantly, but not exclusively, journalistic) literature on the subject. It is a position that has been subjected to criticism, clarification and reevaluation. By way of example, I would argue that participants on Internet forums are not as equally positioned as those who set up the forums. The participants are subject to possible deletion of their posts, censorship of their ideas if they engage in flaming or trolling activities, and or outright banning of their IP addresses from the website. Moreover, inasmuch as the participants are users of these forums and not the creators or owners, they cannot expect equal representation of their opinions; neither is there a guarantee that their views will translate to social change or political action in the real world. An even bigger challenge is the economic power needed to gain initial access to these forums. While computers and Internet modems are now more affordable, ongoing maintenance costs such as monthly Internet connectivity fees, and literacies and skills - in the form, say, of the language proficiency needed to engage in debates on political forums - means that in some cases access to such forums will not be viewed as an economic or socio-cultural priority, which will limit participation.

Foucault's theories with regard to discursive formations and practices, and the interrelationship between discourses, forms of knowledge and power, are equally relevant and useful here. 
Foucault argues that discourses are not just a collection of signs, but "practices that systematically form the objects of which they speak" (Foucault, 1972, p. 49). What this means is that the events, sites, organizations, rules, infrastructure, technologies and bodies that constitute the context, materiality and the visual and emotional realities of everyday life are experienced and rendered explicable and meaningful by way of the discursive frames applied to them. We can elaborate by considering what exactly is at stake in Foucault's statement on discourse. Frow writes that:

Each element of this sentence matters: discourses are practices in the sense that they carry out an action; they are systematic because they are relatively coherent in the way they work; they are formative of objects in the very act of speaking of them, not in the sense that they create objects out of nothing but in so far as they build a weight of meaning around the categories of the world. Discourses ... are performative structures that shape the world in the very process of putting it into speech (Frow, 2015, pp. 17-18).

Discourses are not simply a reflection of something that is already there, or a re-presentation of existing meaning and form; rather they determine how we experience, order and evaluate the world. For Foucault, we live within and experience a world that is given to us by and through discourses; and subjects are necessarily shaped, constrained and disposed towards thoughts and actions through their immersion in, and their incorporation of, the discourses of cultural fields such as school, the family and, most particularly, the media. 
For Foucault, discourse facilitates the workings of power. To be able to name, categorize or label someone or something is to exert power and to produce material effects. Foucault's concept of discourse is useful for assessing Internet forum activities, because these forums are often employed by their owners to further the agendas of the state or other powerful groups. Perhaps even more to the point, Foucault's notion of (what is involved in) discourse constitutes a very useful, and even necessary, counter-discourse to the current doxa that represents the Internet as somehow free or removed from conventional (that is to say, offline) cultural political deliberations. Some scholars draw attention to how politicaleconomic interests of new media owners, vis-à-vis the ownership structure and need for advertising revenue, tend to override or compromise the role and status of the Internet as a significant public sphere site (Jensen, 2003; Remaley and Dineen, 2007). The pull/push element, upload/download of documents, the email system, social networking and instant chat, video conferencing, and phone messaging: all these function to put Internet technology within the reach of more citizens than traditional media such as radio, television, newspapers or magazines.

However the Internet is still mostly used for commercial activities; hence, various forms of capitalist transactions tend to dominate the discourse about the viability of Internet forums to effect change in the political process. Moreover, and as recent studies demonstrate (see Frow, 2015), forms of offline cultural capital and (by extension) power differentials are absent once people move to, and communicate on, the Internet. To consider one example, the position of privilege that is associated with capital-rich socio-cultural groups, and the kinds of communication and cultural literacies that tend to manifest and naturalize their 
sense of distinction (Bourdieu, 1989), may not be as marked as they are in offline contexts, but they continue to inhabit online communication contexts and practices, and to inflect which groups are constituted and recognized as knowledgeable and 'supposed to know' about public sphere issues. For Emden and Midgley however, "even if the present state of politics might appear dismal, historical precedents give credence to the potential viability of a public sphere" (p. 5). Additionally, Foucault's theories of surveillance challenge the utopian rhetoric of horizontal ownership and universal access. The head office of the Internet police chief organization ('Internet Corporation for Assigned Names and Number', 2012) is based in the United States of America. Threats of censorship often arise in Asian and Middle Eastern countries, especially during political protests. Further, it is relevant to enquire as to how women in some non-Western countries navigate the challenges of Internet access, including distinct restrictions some countries put in place to prevent the exchange and dissemination of information.

With regard to Habermas' theories about common interest, Foucault insists that discursive formations and power differentials influence or shape public sphere actors, activities and developments. Essentially Foucault argues that subjects are always caught up in, and produced through, discourses and discursive formations: he argues that discourses and discursive formations, and the meanings and dispositions they produce, are constituted through, and by, power relations. His position is that it is utopian to presume that determining what constitutes issues of common interest will be arrived at naturally, logically or in a disinterested way. Additionally, such determination of issues and their subsequent deliberations would also be influenced by the extent to which some groups have 
more economic, socio-political or cultural capital than others. Hence, the ideal of equality espoused by Habermas and Fraser has to be viewed as utopian; that is, as an ideal which, like the Gricean Co-operative Principle, can be cited by groups as something which civil society 'should' be based upon.

For Foucault, the public sphere ideal of reflexive deliberation is utopian, because all thought and deliberation is to some extent delimited by power relations. So the concept of truth, for instance, can only be understood as an effect of what he refers to as the powerknowledge nexus (Schirato, Danaher and Webb, 2012). In other words what comes to be accepted and read as truth is always both arbitrary and motivated: it is arbitrary in the sense that it is a particular position or idea that establishes itself as universal, and it is motivated in that it achieves this status via the workings of power. Foucault sees power as a pervasive force that authorizes and produces what comes to be recognized as knowledge: his position is that, as a consequence of the role of power relationship in influencing communication practices and the meanings that arise from them, the notion of determining an autonomous or natural 'truth' in public discourse is untenable. Foucault argues that there is a need to detach "the power of truth from the forms of hegemony, social, economic, and cultural, within which it operates" (Foucault, 2000, p. 133). For Foucault, truth and power are linked together and mutually dependent.

In Foucault's discussion of language and epistemology, he argues that what is considered real or true is the product of discourse. Foucault's position here differs, to some extent, from Hall's position that "reality exists outside language, but it is constantly mediated by, 
and through, language, and what we can know and say has to be produced in and through discourse" (Hall, 1993, p. 95). Foucault's position contrasts with Hall's in that the latter positions subjects as exercising greater agency at the moment of decoding messages, although they may not even know they have this agency. However, at the same time their positions are commensurate, in that Foucault does not 'bracket off' or deny that there is anything outside discourse; rather, he suggests it is only in, and through, discourse that the world is recognized. This is an important distinction. As Schirato et al. write, for Foucault the subject:

Is not simply or necessarily an effect of discourse: Foucault identifies a process or logic whereby what he calls the 'critical attitude' ... arises out of the creation, dissemination and deployment of those (historically specific) ideas, imperatives and dispositions that come to constitute or contribute to a particular grid of intelligibility (Schirato et al., 2012, p. 186).

The key aspects of Habermas' public sphere theory that are problematized by Foucault are that participants are to be reflexive by critically examining their assumptions and situating their cultural values and interests within the larger social context, and that they are to be sincere, hence able to indicate their true intentions in relation to the common issues under deliberation. These expectations by Habermas are performatives: the expectations would vary from one culture to another as they may be actualized in some public sphere deliberations while it will be impractical to expect them in others. There is, however, another way in which the question of reflexivity is made problematical on the Internet, and 
that is tied up with the proliferation of intensely personal communications that can overload discussions and drive them away from any consideration of a wider socio-cultural dimension. This is not so much a case of divorcing the private from the public (which would be untenable), as pointing to the manner in which the personal is often produced as independent from wider communal contexts.

By way of example, consider the way some people use blogs, Twitter, or Facebook: private confessions - about family life, individual lifestyles, sexual desires, educational pursuit, and leisure goals, among many others - proliferate on these platforms. Such private confessions can be contrasted to the critical appraisals of issues of common interest that Habermas envisions as the driving force of public sphere deliberations. This atomization of the subject is arguably derived from capitalist discourses that emphasize and normalize the notion of the individual who is outside, not linked to, socio-cultural forces and discourses. The Internet as discursive apparatus produces people as 'disposed' subjects, a position that shares a certain amount of commonality with Marxist and neo-Marxist theory, most particularly Frankfurt School scholars such as Adorno and Horkheimer (1972). As we shall see, however, Foucault does not subscribe to the Frankfurt School position regarding the relation between cultural production, ideology and cultural consumption; his position is more open and flexible, in that he allows both that certain groups (such as intellectuals) are disposed to think critically about and against discursive regimes and their naturalized meanings; he also makes the point that those discursive regimes necessarily produce their 'other', or groups that cannot be integrated into or which stand outside normative categories of subjectivity. 
The Public Sphere and Intellectuals

In an interview with Foucault (2000), he discusses intellectuals and their role in resisting power. For Foucault, the intellectual is a person who uses knowledge, competence, and relation to the truth in the field of political struggles. He distinguishes between the universal and the specific intellectual. The universal intellectual developed from the jurist notable/law, while the specific intellectual came from the sciences in the $19^{\text {th }}$ century (Foucault, 2000, p. 128). Foucault argues that the "left" intellectual used to be the master of truth and justice. On the rise of "specific" intellectuals, he says it is no longer about universal intellectuals but intellectuals in "specific sectors", and attempting to make changes in places where they have influence and power. For Foucault:

Intellectuals have become used to working not in the modality of the "universal," the "exemplary," the "just-and-true-for-all," but within specific sectors, at the precise points where their own conditions of life or work situate them (housing, the hospital, the asylum, the laboratory, the university, family and sexual relations [and may I add, in online Internet forums?]). This positioning has undoubtedly given them a much more immediate and concrete awareness of the struggles in various communities. And the intellectuals in turn have met here with problems that are specific, "nonuniversal," and often different from those of the proletariat or the masses (Foucault, 2000, p. 126). 
Foucault also points out that it is not just writers who can function as intellectuals:

Since the time when each individual's specific activity began to serve as the basis for politicization, the threshold of writing, as the sacralizing mark of the intellectual, has disappeared. And it has become possible to develop lateral connections across different forms of knowledge and from one focus of politicization to another. Magistrates and psychiatrists, doctors and social workers, laboratory technicians and sociologists have become able to participate - both within their own fields and through mutual exchange and support - in a global process of politicization of intellectuals (Foucault, 2000, p. 127).

Dahlgren (2012) and Gamble (2004) have explored Foucault's notion of the work of intellectuals further to discuss how they function in technological domains. For some of these intellectuals, there is a tendency to gather around already established positions as opposed to being distinct and unique in their perspectives on specific issues. Perhaps due to the forms of digital technology and the ways most platforms for these discussions have already established agenda, which may not be overtly stated, the intellectuals who engage on the platforms tend to reinforce the opinions of the owners of the platform rather than use their presence there to challenge the status quo. This phenomenon is occurring in new media spaces in the same ways that they manifest on old media platforms. Take for instance the ways in which Fox News channel commentators and panel members tend to be strongly conservative and express views that align with the ideas of the show hosts, who sometimes handpick those contributing on their shows. These commentators and panel members are 
presented as experts in their field and viewers presumably assume that they are expressing independent opinions but what is not overtly stated is that these are often paid appearances. In the same way, such 'expert' opinions now proliferate in online spaces, infiltrating the new media forums that were hitherto assumed to give voice to more citizens and opportune emerging and budding intellectuals to steer discussions in unorthodox and critical directions.

Moreover, Foucault suggests that this development of the specific intellectual is tied in with, important to, and a valuable aspect of, the academic field. The influence of the university is strengthened because it now "produces" more professionals who are questioning authority and power. He focused on Western intellectuals, specifically in the political field. He however noted that there is a danger facing the specific intellectual, which is that their activities, discourses, arguments and challenges are both removed from, and have no obvious way of gaining access to, the public sphere sites of the mainstream and popular capitalist media. Foucault notes that there is a plurality of resistances (Foucault, 1990a, p. 96). He dismisses the notion of power as something that is imposed from above; instead, power is always something that is "negotiated" between the people and those governing them:

There is not, on the one side, a discourse of power, and opposite it, another discourse that runs counter to it. Discourses are tactical elements or blocks operating in the field of force relations: there can exist different and even contradictory discourses within the same strategy; they can, on the contrary, 
circulate without changing their form from one strategy to another, opposing strategy (Foucault, 1990a, p. 101).

Furthermore, he argues that power presents ideas that favour its agenda as universal truths that are ultimately beneficial to all (2000, p. 131). Foucault suggests that we need to focus on changing how truth is produced in political and economic relations. There is a need to detach "the power of truth from the forms of hegemony, social, economic, and cultural, within which it operates at the present time" (Foucault, 2000, p. 133). Since truth is already bound up in power, they have become one and the same; therefore, the project for intellectuals is to work on detaching "the power of truth" from the various forms of relations between those in authority and the people they govern.

Power, Subjectivity and Technology

Foucault argues that power does not operate as a force that needs consensus, or in terms of the overt domination of the governed by the governing; rather, it functions by, and through, the ways in which subjects are produced, disciplined and regulated (Foucault, 1990a). In this account, certain forms and orders of discourse, which are legitimized by bodies of knowledge emanating from, or associated with, fields such as psychology, science, sociology and health studies, purportedly identify, describe and provide rationales with regard to the 'truths' of human subjects and society. However, in the process of disseminating and establishing their 'descriptions and findings', they produce and naturalize their versions of the world and everything in it. For Foucault, power - in the form of 
authorized discourses, stories, accounts, explanations, templates and exemplars - circulates through the socio-cultural field, producing different manifestations and consequences in its wake. Clearly certain groups are more likely to be able to associate themselves with, or facilitate the dissemination of, authorized discourses and texts, particularly in the capitalist media. In this way, "power is not something that is acquired, seized, or shared, something that one holds on to or allows to slip away; power is exercised from innumerable points, in the interplay of non-egalitarian and mobile relations" (Foucault, 1990a, p. 94). He contends that subjects are always "inside" power: that is to say, the world we live in and make meanings of is more or less given to us by and through the workings - and discourses and texts - of power (Foucault, 1990a, p. 95). Power and knowledge are intertwined; subjects are constantly negotiating their places in relations of power.

Foucault (1990b and 2000) refers to relations of power as extending beyond the limits of the state; in fact, he argues in another context (Foucault, 2007) that the state itself, and its naturalized place within social science and political discourse and analysis, is an effect of power. What looks like the origins or places of power or value - the state, conventional political systems and forms of government, abstracted notions such as 'freedom' and 'universal rights' - are the product of specific socio-cultural and historical relationships and trajectories of power. For Foucault, "what makes power hold good, what makes it accepted, is simply the fact that it doesn't only weigh on us as a force that says no; it also traverses and produces things; it induces pleasure, forms knowledge, produces discourse" (Foucault, 2000, p. 120). Power is seen as a multi-faceted force whose legitimacy is found in the notion of what is considered true. Power should be considered as "a productive network 
that runs through the whole social body, much more than as a negative instance whose function is repression" (Foucault, 2000, p. 121). It is not so much about what power does or can do to people, but how people experience and live in power in relation to what they consider to be true and necessary for their social conditions. Foucault asks that we consider how governments are able to make subjects of the people they govern.

Foucault's work on the relation between power and subjectivity is in a sense both an extension of, and a reaction against, the theories of the 'Frankfurt School'. The 'Frankfurt School' theorists are usually more specific about, and insistent upon, the ways in which capitalist cultural forms and texts manipulate and direct the responses and attitudes of audiences. They maintain a strong theoretical commitment to the notion that cultural production is to be found at the level of what Marx (1978) refers to as cultural consumption, which is associated with the argument that subjects are complicit in their own mass deception.

Emden and Midgley advise that, "a working public sphere is always limited" (2013, p. 6) by time and other resources that results in its disintegration soon after it is formed and potentially after it has achieved some of its objectives. The ideals of the public sphere as an autonomous metaphorical space for discussion and deliberation benefitted largely from the form and context of old media - newspapers, magazines, pamphlets, radio and television that allowed mostly linear systems of communication with minimal feedback. However, new media - Internet sites, social networks, blogs, and forums - are potentially tools that can be used for undermining the commercialization, concentration, and control of the media, 
which was a major concern for Habermas; he warns, for instance, of a "refeudalization" of the governance process (Habermas, 1974, p. 54). These new media are also useful for influencing the form and content of democratic governments and capitalist controlled cultural sites in most democratic societies. Given these theoretical prescriptions, to what extent is it possible to talk about or presume agency with regard to online activities? Hall (1993) provides a somewhat different approach to both Foucault and (most particularly) the Frankfurt School, arguing that when confronted with media messages, audiences are able to adopt any one of three reading positions - preferred, negotiated and oppositional - at the moment of decoding. Utilizing an approach commensurate with that of Hall, Chen (2011) examines how Twitter use gratifies the users' need to connect with others. Applying uses and gratifications theory, Chen investigated what people did with media, as opposed to what the media do to people. Uses and gratifications theory suggests that individuals have, and do exercise, agency. The theory shows that people have "a need to affiliate or feel a sense of belonging" (Chen, 2011, p. 756). One of the ways to fulfill this need is arguably by "capitalizing on the web's potential for interaction that is absent in more static communication forms such as a print newsletter or newspaper” (Chen, 2011, p. 757). Ruggiero further points out that "the web offers the potential for interactivity" (in Chen, 2011, p. 757).

The issue of the intensification and proliferation of highly personalized communication practices and narratives can be said to further problematize the efficacy of the Internet as public sphere in yet another regard, specifically in terms of the question of information overload. An analysis of this issue will benefit from Innis' and McLuhan's theorizing about 
the concepts of time and space, as well as the deterministic nature of a medium of communication, respectively. The average individual has so many media, among many other things, constantly competing for her attention. This practice has an impact on whose posts are read, how the posts are chosen, and ultimately whose opinions are privileged on Internet discussion platforms. Such a reflection would help in further interrogating class and social relations, and how these relations are shaped by different discursive regimes.

\section{Reflexivity and Knowledge}

One of the accusations leveled at theorists of the public sphere, and at Habermasian theory in particular, is its failure to appreciate or interrogate the notion that whatever does come to constitute public sphere sites, spaces and activities is never arrived at in any neutral way: rather, these outcomes are always already derived from a set of discursive formations, power relations and socio-cultural institutions, practices and dispositions that are largely masculinist in character. Tully (2013) argues that:

Public spheres and their vocabulary are various and variable because they are negotiated practices. Citizens do not engage in public spheres only to form public opinions... they also engage in the critical and reflective activity of calling into question, testing the adequacy, negotiating and modifying the given rules, scripts, roles, and relationships of the public spheres in which they act (p. 171). 
That this oversight can pass without attention is partly a consequence of the extent to which what we might call gender politics has been naturalized, and consequently transferred to, and incorporated within, the (general socio-cultural) habitus as a way of seeing, recognizing, ordering, and making sense and meaning of the world, most certainly with regard to men, but also to no small extent with women. As Bourdieu writes:

The division between the sexes appears to be 'in the order of things', as people sometimes say to refer to what is normal, natural, to the point of being inevitable: it is present both ... in things (in the house, for example, every part of which is 'sexed'), in the whole social world, and - in the embodied state - in the habitus of the agents, functioning as systems of schemes of perception, thought and action ... It is the concordance between the objective structures and the cognitive structures, between ... the course of the world and the expectations about it ... that makes possible ... the 'natural attitude' or 'doxic experience' ... This experience apprehends the social world and its arbitrary divisions, starting with the socially constructed division between the sexes, as natural, self-evident, and as such contains a full recognition of legitimacy (Bourdieu, 2000, pp. 8-9).

This situation largely defines and delimits the public sphere. However as Pateman argues:

Since the late seventeenth century, feminists have been pointing out that almost all political theorists have in fact, explicitly or tacitly, upheld 
patriarchal right ... However, none of this has been sufficient to convince all but the smallest minority of male political theorists or activists that patriarchal right still exists, demands theoretical scrutiny and is as worthy an opponent as aristocratic, class or other forms of power (Pateman, 1988, p. 19).

The masculinist and patriarchal nature of the theories about, and debates regarding, the public sphere is in a sense almost as significant an issue as the ways in which masculinist power delimits access to the public sphere based on the basis of the 'naturalizing' of gender politics. This is where academic or theoretical reflexivity has an important role to play: one of the objects of those processes that produce 'knowledge' is the nature of knowledge itself. As Butler writes:

The question of who and what is considered real and true is apparently a question of knowledge. But it is also, as Michel Foucault makes plain, a question of power. Having or bearing 'truth' and 'reality' is an enormously powerful prerogative within the social world, one way that power dissimulates as ontology. According to Foucault, one of the first tasks of a radical critique is to discern the relation 'between mechanisms of coercion and elements of knowledge' ... Knowledge and power are not finally separable but work together to establish a set of subtle and explicit criteria for thinking about the world (Butler, 2004, p. 27). 
The same characterization can be applied to other forms of cultural politics, such as those involving race and colonialism. hooks argues that white academics should acknowledge the role of their ancestors in perpetuating slavery and work out in their academic writing a critique of the systemic domination and colonialism that was visited on blacks in America, especially during the Jim Crow era (hooks, 1990). She notes that such reflexivity as is required to fulfill this mandate is possible, especially in the area of cultural and critical theory. hooks suggests that the collaborative work between feminist scholars and activists enabled the interrogation of issues of racism and colonialism. These interrogations, she contends, were fiercely fought for by black women and women of colour (hooks, 1990, p. 124). She is careful to note that disciplines such as cultural studies - which was largely conceptualized, and taught by, white men - is gradually being privileged and receiving legitimacy over programs such as African-American and Third World studies, two key areas that have focused extensively on issues of racism and colonialism. hooks makes the point that she is not against cultural studies - in as far as it further advances the course of critical scholars, including feminists - but that the stamp of legitimacy it receives, because of the white men administering it, further re-colonizes the pioneering scholars who laid the preliminary groundwork for women's studies.

Decolonizing Methodologies extensively detail the tools Western cultures use to continue their colonial legacy, both domestically and internationally (Smith, 1999). One of the major ways in which this phase of colonialism is continued is in the dissemination of knowledge. The questions of who possesses what knowledge, which knowledge is privileged and documented, as well as who is considered an authority on any particular issue, are all 
relevant to understanding the ways in which forms of knowledge help perpetuate inequity and injustice. In a similar vein, Becker argues that research and researcher are related, and that there is no such thing as value-free research (Becker, 2004, p. 23). Starting with the notion of bias, he points out that the accusations often arise in situations where hierarchical orders are likely to be disrupted. In examining the issue of bias in what he terms "apolitical situations", Becker notes that stakeholders in such situations are often not fighting to trade places, while in the other case (a political situation), there are actually attempts by subordinate parties to unseat the "superordinates" - those who are in positions of power and authority over them (p. 24). His article is illuminating in its sketch of why sociologists are accused of bias in their research work. Becker's discussion about the hierarchy of credibility, and how this reinforces the imbalance between superordinate and subordinate groups, helps provide an understanding of how sociological knowledge is always tied in with cultural politics.

Conclusion

The first two sections of this chapter gave a brief overview of the three key elements of Habermas' public sphere theory and its main components - accessibility, discursive inclusivity and equality, and autonomy - as well as the central elements of communicative action theory. The sections indicated the problems inherent in the ideals espoused by Habermas. Fraser's critique of Habermas and Pateman's critique of social contract theorists show how the Habermasian criteria are not 'real' things, but performatives that cannot be universally sustained; hence they require a reconsideration for public discourse and 
deliberations, especially in relation to digital forums. Despite its limitations, public sphere theory is still considered useful for assessing communicative practices in public domains: what is required, however, is an incorporation of the consideration of how various factors and contexts, for instance economic relations and access to or ownership of the means of material and cultural production, inform and influence public sphere forms, sites and levels of activity.

Marx reminds us that the forms of class and social relations that occur in the nodes of production show that ideals of accessibility and inclusiveness are not completely attainable: those who have the means of production are able to influence and determine what the technology is used for and by extension, can covertly limit who have access and which voices are privileged in the course of debates about issues of interest in the public sphere. Foucault's concepts of discourse and power relations further build on Marx's class and social relations to show how individuals are constituted, influenced and somewhat limited by their status and skill when engaging in deliberations about issues of common interest. He shows that the limitations or lack of language skill, when combined with little or no knowledge of the subjects being discussed, further disenfranchises and limits what an individual can do once they have access to the Internet and or the public sphere.

Unlike traditional media, new media provides opportunities for extensive interaction among citizens although these interactions are not necessarily used primarily for the purpose of civic engagement. However the availability of the tools and the take up of the opportunities among certain groups and cultures - especially during periods of contested argumentation 
and distribution of resources - show that the potentials of new media technologies can be harnessed for the benefit of citizens and to further improve civic participation. Meanwhile, Butler, hooks and Smith draw our attention to questions about the documenting, disseminating, archiving and most importantly privileging of certain forms of knowledge over others. These questions require a certain level of reflexivity that asks scholars to do a self-critique that will enable them to see how such practices of power that they analyze and study in society are also strongly represented in academic circles and by extension, can further create inequity and injustice.

In summary, Marx's and Foucault's theories are central to understanding how issues of social and class relations, discourse and power inform public sphere activity. We may find that critical-rational discourse is limited, and perhaps constrained, on WorldPulse.com because the site is an account of real-world deliberations as opposed to the ideal espoused by Habermas. However, to a large extent, participants on the site utilize reason and rationality, two key features of the public sphere and communicative action, if only because they are the 'rules of the game' that the site requires of participants. What takes place on WPc is an extension of critical thought in a micro space with intent to effect changes in macro policies. This critical deliberation contrasts with the use of violence in some other context to achieve the same goals. However, while WPc may enable its participants to be reflexive and engage in rational-critical debate, there is little to indicate that the site is aware of power imbalance among participants, and between participants and owners of the site. Hence, while it appears that it is hard for anyone not to commit to the ideals of the public sphere, the proponents of these ideals need not necessarily actualize or reproduce 
them in their communicative practices.

This chapter has introduced and discussed the theoretical material that will be used in this thesis in order to analyze, contextualize and evaluate the WPc website as a space for promoting public sphere activity. In the next chapter I will provide a critical review of existing literature about the public sphere and communicative action, new media and the public sphere, gender and public sphere activity, social movements and the public sphere, and the global public sphere. 
Chapter Two: Literature Review

Introduction

The literature review for this thesis is structured around five main areas, namely: the public sphere and communicative action; new media and the public sphere; gender and public sphere activity; social movements and the public sphere; and the global public sphere. This chapter will provide an account of the scholarly literature, and the critical debates, relevant to this thesis. Communicative action theory revises some of the aspects of Habermasian public sphere theory that have been critiqued as idealist, utopian, exclusionary and homogenizing. Some of the scholars contributing to this debate include Dahlberg (2001, 2005), Dahlgren (2012), Dean (2003), Gamble (2004), Lunt and Livingstone (2013), Markell (1997), and Volkmer (2014).

The Public Sphere and Communicative Action

In examining the theory of the public sphere and the revisions made to the theory through communicative action, I explore existing scholarship that discusses the relevance of public sphere theory to modern day democracy; the role of public intellectuals and organic intellectuals in the digital public sphere; and some of the new models media studies scholars suggest can aid in better working with communicative action theory to assess communication practices in the public sphere. Some of the issues arising as a result of the revisions made to Habermas' public sphere theory revives the debate about the relevance of 
public sphere theory itself to modern day democracy, and the roles of public as well as organic intellectuals in sustaining deliberative discourse in the public sphere. Habermas initially envisioned the public sphere as a metaphorical space in which deliberation is ideally based on logical reason, and undergirded by consensus. The ideal envisioned by Habermas has since been revised in his later works on communicative action: emphasis is placed more on the discursive practices between interlocutors, and less on the space within which the discussions occur. The normative conditions underpinning public sphere activity, for Habermas, are characterized by six criteria, operationalized by Dahlberg (2001) in assessing the Minnesota eDemocracy listserv.

It has been argued that the systematic exclusion of some groups from communal debates and discussion, the significance of power differentials, and the complexity of the notion of what constitutes a consensus, all render Habermas' theory inapplicable to the challenges of modern day democracy (Fraser, 2009). However, Habermas has expanded his initial conception of the public sphere by introducing a notion of formal pragmatics, which "allows the identification and explication of conditions of argumentation presupposed by participants engaged in communicative interaction" (Dahlberg, 2005, p. 112). For Dahlberg, public sphere criteria are useful for assessing "everyday discursive practices"; he contends that Habermas' communicative action theory has addressed the restrictions found in his earlier conception of the public sphere that was considered homogeneous and exclusionary. He further argues that "The public sphere is now defined by a form of communication and not a particular content" (p. 112). 
For Markel, Habermas has revised his notion of consensus and addressed the glaring exclusion of marginalized groups, especially women, from the bourgeois public sphere. This revision allows for a broader examination of "rational-critical discourse" that occurs in "everyday communicative practices" (Markell, 1997, p. 378). Consensus is not necessarily predicated on any mutual agreement obtained prior to the commencement of discursive action, but rather is worked out, and through, in the process of deliberation. "Just as Arendt points toward the unpresentability of "the sovereign people" in a democratic age, Habermas recasts the theory of democratic legitimacy in a model form ... a democratic politics for which open-endedness is not a failure but a necessary and constitutive feature" (Markell, 1997, p. 394). From this perspective, the legitimacy of the topics of the discourse and the discursive patterns and practices of the public sphere are not limited to pre-agreed and predetermined agendas, but also include issues of contestation, some of which interlocutors may not entirely agree on or successfully resolve.

Habermas' revision of the notion of the public sphere now includes a critical edge that enables an analysis of social phenomena beyond the level of historical abstraction. $\mathrm{He}$ recognizes the actual practice of communicating - rather than the space within which communication takes place - as a central element of the public sphere. Lunt and Livingstone state that, "Many adopt the pragmatic view that the concept [of the public sphere] establishes a good starting point, a framework against which to test one's ideas and assumptions" (2013, p. 91). From this perspective, Habermas is no longer committed to the notion of a unitary public sphere, but rather accepts that contested communication occurs in multiple spheres and the nature of the sphere - for instance its link to state or economic 
power - does not necessarily delegitimize the value of the communicative practices. Habermas' concern about the autonomy of public sphere sites have also been revised to show that certain institutions, for example the law, do have a role in facilitating deliberation and civic engagement. He acknowledges the need for "inclusivity, diversity, identity [and] the end of consensus government" (p. 95). Although Habermas still subscribes to the theoretical value of the original idealized conception of the public sphere, there is a recognition that the public sphere in its initial conception is not a social reality. Hence, the ideal public sphere is still the ultimate goal while existing public spheres are considered sufficient to serve the function of enabling and furthering communicative practices for citizens. In these spheres, the politics citizens engage in is fluid, as it is often present in every facet of life while institutions equally have a role in deliberation and public engagement.

Wilson (2013) argues that "conversation and public reasoning, and the tension between individual freedom and the authority of the state are central elements in the public sphere" (p. 20). There is a perceived crisis of democracy in certain parts of the world (Dahlgren, 2012; Gamble, 2004), and this crisis can be traced to a decrease in citizens' involvement in the democratic process; that is, in terms of a decline in voting and public engagement. This decline in political participation comes with grave consequences. Recent examples include Britain's Brexit referendum, whose outcome was a shock to many young British nationals who later confessed to not voting because they assumed their vote would not make a difference in the outcome of the referendum. Many British youth living in London 
expressed publicly, on the day after the vote result showed that Britain would leave the European Union, that they would rather Britain stay in the EU.

Gamble uses Gramsci's notion of the public and organic intellectuals to point to a supposed decline in participation of 'the public' in the democratic process. One of the reasons for this crisis is "the failure of intellectuals to understand the nature of the public domain and their role in sustaining it" (Gamble, 2004, p. 42). Public intellectuals, because of their sociocultural status and capital, are sometimes able to influence majority action. As subjects who interrogate and critique power and are committed to a critical public domain, Gramsci's organic intellectuals should pursue an agenda of reform fervently: "The public domain [is] a sphere of political and ideological contestation, in which attempts to set agendas and define identities and the public interest, assemble coalitions and alliances, and make interventions become all-important" are constantly negotiated (p. 43).

Online space offers opportunities and avenues for public intellectuals, some of who may still be developing their skill of engagement with political and ideological issues (Dahlgren, 2012). These organic intellectuals are not restricted to their areas of expertise, but are also able to influence policy and politics as public intellectuals engaging in the public domain (Dahlgren, 2012; Gamble, 2004). Emden and Midgley (2013) inform us that "Publics are historically conditioned and socially concrete "things;" that is they emerge or are dissolved in specific locations; they are shaped by the technologies and material culture to which they are subject, and they also serve the production of knowledge" (p. 3). For the goal of revitalizing democracy to be achieved, there have to be channels for public intellectuals' 
ideas to find expression, without attempts to stifle them or compromise their stance. However, it appears that rational-critical debate is not priority for most people engaging in discussions in contemporary digital public fora (Dahlgren, 2012; Volkmer, 2014).

As a response to both the supposed decline in democratic participation and the popularity of digital sites (including social media) of public discussion, contemporary scholarship has proposed alternative theories to Habermas' formulation of the public sphere. One of these contributions is actor-network theory, developed by Michel Callon and Bruno Latour (in Dean, 2013); another alternative is public horizons, proposed by Volkmer (2014). Rogers and Marres have expanded on actor-network theory by introducing the concept of "neodemocratic issue networks" as a way of analyzing issues and events on the Internet (in Dean, 2003, p. 97). In this context, Internet technology is not considered neutral or passive, but rather is regarded as an active context that impacts on and influences other technologies; this is relevant, for instance, when analyzing communication practices on social media platforms such as Facebook or Twitter.

Volkmer (2014) identified three critiques of modern deliberative ideal speech situations. The first critique argues that consensus is temporary and shored up by hegemony but, in this context, exclusion is unavoidable. In the pursuit of rationality, individual and subjective passions and affects are sidelined. The focus is on the types of activities and events - "practices" - that are occurring in a site/space of deliberative discourse rather than on the logic and rationality of arguments. Secondly, she contends that 'identity' and 'reflection' are important elements of deliberation. National and transnational exist 
simultaneously, and both interests can be represented in a public sphere, with consideration for specific group interests that feed into broader public interests. Thirdly, she writes that 'discourse ethics should incorporate the 'situating of the self', the individual within deliberative discourse" (Benhabib in Volkmer, 2014, p. 172). Deliberative discourse in the age of new media is not focused on an outcome underpinned by consensus, but is rather about engaged citizens being reflexive and expressing their reflections in a collective transnational sphere. As Volkmer (2014) argues, public communication is now occurring in the 'lifeworld': "It is the consensus orientation based on the perception of common public 'good' which shifts towards consensus orientation in the perception of shared public 'dimensions' in the time of public interdependence” (p. 169). This sphere is linked to citizens' potential to influence political discourse and those in positions of power through the Parliament, city council and local boards.

New Media and the Public Sphere

The accelerated development in new media and digital technologies over the last two decades has meant that people engaging in democratic discussions and practices have more tools at their disposal for putting their views forward. Some of the issues arising about new media and the public sphere include a critique of the affordability of digital media; the opportunities it presents citizens for challenging traditional media's agenda-setting function; new media's role in allowing or constraining access to the public sphere; and the use of mobile technology for political participation, which has gained more prominence with the expanded visibility Internet technology enables. Citizens' increased access to, and 
use of, new media technologies has resulted in the disruption of traditional media's agenda setting functionalities (Frow, 2015; Papacharissi, 2009, 2010; Tilley, 2005; Tully, 2013; Volkmer, 2008; Wilson, 2013). For instance, Wilson argues that, "technology and blogs have the potential to allow the individual to participate in the public sphere and to have an impact on it" (2013, p. 21) while Tully (2013) observes that, "a customized (and often customary) public sphere in one place speaks to the similarly situated public audiences all along global networks of production, consumption, and volunteer activities, and their voices are amplified by electronic networks of e-mail, YouTube, Facebook, and so on" (p. 188).

Part of the challenge of digital and other new media is that they tend to 'rewrite' the rules and conventions of communication exchanges. John Frow, for instance, has pointed out that it is difficult to delineate clear generic boundaries within computer-mediated communication (Frow, 2015). Some of the challenges Frow identifies include: issues of taxonomy and distribution, distinction between blogs, feminized versus masculine bloggers, and blog's ancestry. Blogs can function as links between static pages and discussion forums, thereby forming communities of value although "narrowcast form does not engage with a broader public sphere" (p. 156). Frow takes the position that "the blog is always public speech, even when the notion of a homogeneous public sphere has effectively disappeared" (p. 156). One of the functions that the subgenre of forums, which has its ancestry in blogging genre, enables is the opportunity for forum members to redefine what constitutes news (by challenging gate keeping), and what they should think about (challenging agenda setting theory). The perceived presence people feel when engaging in electronic 
communication is just a "fantasy" (p. 156). Comments from often excluded voices can shift the angle of the story or narrative to highlight other missing elements of discourse, such as those who are directly affected by the issues but were not necessarily consulted before the story was written and published (Tilley, 2005).

To what extent do electronic media facilitate or hinder access to the public sphere; and by extension, does digital democracy influence modes of governance? New media technology, especially the Internet, has arguably created more opportunities for citizens to engage in the political process. There are scholars on both sides of the divide about the Internet's potential to facilitate public spheres. However, communication scholars - including Blumler and Gurevitch (2001) - argue that research about new media should move away from the utopia-dystopia dichotomy, and focus more on its dialectical potential. Digital inequality asks questions about cultural and social capital, a step above the questions of economic capital that is often the focus of studies about digital divide. Bourdieu's discussion of the complexities of social, cultural and political capital is useful for exploring these issues. Skill is a significant form of cultural capital that is linked to education and social status. By way of example, in Niger the skills needed to use the Internet to participate in civic engagement online is tied to the issue of the availability and distribution of cultural and social capital among Nigeriens (Alzouma, 2013). Data from Niger show that Nigeriens are doubly restricted in the digital landscape in terms of both economic and social and cultural factors (Alzouma, 2013, p. 300). Rural-urban digital divide is strongly associated with poverty and illiteracy in Niger; urban city dwellers have greater access to digital technology than those in rural areas. However among those with access in the rural areas, 
digital inequalities - such as knowledge of how to use some of the applications - are widespread.

Skills and competencies allow agents to utilize digital media more effectively. Economic, cultural and social forms of capital are important factors here, and the problem of the inequalities in these forms of capital is accentuated when the issue of political capital is added to the equation (Emden and Midgley, 2013; Wellman et al, 2001; Wilson, 2013). In their study of visitors to the National Geography Society website, Wellman et al. reviewed the utopia-dystopia dichotomy that has become a common feature of new media literature. Unlike most scholars who research the use of the Internet, Wellman et al.'s research examined "people's Internet use in the broader context of their face-to-face and telephone communication" (Wellman et al., 2001, p. 438). They critically analyzed visitors' organizational and political participation at an online level. They propose the 'supplement hypothesis', which argues that Internet use supplements, rather than replaces, other communication tools, most notably face-to-face and telephone communication. This theory can be extended to cover people's participation in political activities. Those participating in political activities online most likely already participate offline: frequency of use of Internet technology was linked to level of involvement in politics online (Wellman et al., 2001). Based on this premise, they suggest that "Internet use increases participatory capital. The more people are on the Internet and the more they are involved in online organizational and political activity, the more they are involved in offline organizational and political activity" (p. 450). What this means is that access to Internet technology can potentially put an already engaged citizen at an advantage to further participate in political deliberations that 
are occurring online, some of which may be far removed from the person's immediate locale or community.

Radovanovic (2013) uses Weberian social stratification theory to explore the challenges of ICT use and uptake in higher education in Serbia; specifically, he looked at the use of social media in educational institutions. Weber's social stratification theory identifies exploration and investigation of knowledge gaps and digital literacy skills - in addition to owning a computer or having access to the Internet - as aspects of the digital divide. These knowledge gaps are consequences of people not knowing that electronic resources are available, or of not having access to those resources, even though they have Internet access. There is a generational gap that is informed by employment status and rank - for instance, professors versus junior faculty versus students. This gap affects opportunities for collaboration using social media, and digital technology more generally, in the curriculum.

Public sphere participation always has a political dimension of some form. In a review of public service broadcasting models for engagement in the public sphere, Born (2013) observed that most Western societies have seen rapid changes in their composition that have resulted in the adoption of multiculturalism and cosmopolitan values mixed with individualism while others still try to hold on to the ideals of a unitary national identity. These changes were a result of migration and they have challenged the unitary, fixed national identities that public service broadcasting was originally modeled to uphold. Born connects this changing face of some Western countries in the context of multi-ethnicity, multi-culture and multi-faith to the changing nature of the media. Moreover, power 
differentials can influence citizens' ability to participate in civic activities through the media. Dahlgren (2014) rejects the idea that interacting online via social media platforms like Facebook and Twitter constitutes civic participation. However, the notion of civic engagement needs to be considered more carefully and openly. The civic engagement typology introduced in chapter eight of this study is one way of reconsidering what can be understood or identified as civic activity and engagement. Civic activity and participation needs to be viewed as one piece of a more robust, complex, dynamic and fluid aspect of social, cultural and political engagement, as is the case with the civic engagement typology, rather than something prescribed by abstracted criteria.

Dahlgren is critical of the function and status of the 'Like' button on Facebook: he sees it as a form of socio-cultural participation where nothing is at stake, and where people's responses are often arbitrary and inconsequential. However he does not engage fully with the possible reasons why some Facebook users may limit themselves to just liking a post, rather than writing about and responding to them in a more committed way. He does not consider, for instance, that time, literacy and technological competence may be factors that limit how people respond to comments or posts.

We made the point that Habermas' notion of the public sphere is metaphorical and idealistic, rather than material and practical. The inclusion of a consideration of the uses of Internet technology goes some way to redressing this problem: it enhances our understanding of the public sphere as a site of significant communicative action (Boeder, 2005; Volkmer, 2015). What is "central in Habermas' thinking is the notion that the quality 
of society depends on our capacity to communicate about, and debate and discuss, issues and topics that are of social, cultural or political relevance. For Habermas, reason is a crucial element of these forms of public sphere communication and discussion" (Boeder, 2005, p. 2). This is contrary to Foucault's position that problematizes the notion of reason; or again, which draws attention to the point that what might be read or become authorized as reason is tied to contexts and forces of power.

There is also a strong and sustained criticism of the supposed public sphere function of the Internet, most particularly in terms of the charge that the Internet is characterized by commodity consumption and materialism, rather than reasoned and critical citizens (Tully, 2013). There is also the criticism that the Internet constitutes a form of surveillance that will limit citizens' involvement in communities, unions or interest groups. Another position taken by critics is that although Internet technology may constitute a leveling ground for people from diverse strands to gather and deliberate, discussion on most sites is often dominated by high status groups who often set the agenda for debates, and thus favour their own interests (Papacharissi, 2010). However theorists and critics such as Boeder (2005), Dahlgren (2012), Emden and Midgley (2013), and Poster (2001) see the Internet as a critical tool for newly emerging organic and public intellectuals, despite the aforementioned issues and limitations.

It has been argued that the Internet can work to disadvantage already disenfranchised groups, and thus effectively reinforce the political and social status quo (Alzouma, 2013; Blumler and Gurevitch, 2001). The argument is that commitment to and engagement with 
the consideration of important public sphere issues and debates runs the risk, on the Internet, of being carried out in an ephemeral and superficial manner; the 'performance' of civic involvement, in some cases, might be dictated by or subject to fashion, trends or 'celebrity' identification. Despite this concern, for most theorists the Internet retains a 'vulnerable potential' for advancing public sphere discussions (Blumler and Gurevitch, 2001; Dahlgren, 2014; Emden and Midgley, 2013; Faris, 2013; Poster, 2001). Blumler and Gurevitch, for instance, have called for the creation of a "civic commons" which would function along the lines of the public sphere posited by Habermas, but be facilitated by digital technology (pp. 9-10). However there is still the issue of the disjunction between the communication protocols and criteria as outlined by Habermas, and the practices that characterize Internet communication. There is little evidence of a culture of civility in online forums: this is often blamed on a lack of moderation of online discussions on some websites, but the question of how reasonable and reasoned moderation is to be provided is a difficult one to address.

The issue of the digital divide is often seen as a constraint on citizens interested in civic engagement. However, the digital divide appeared to be of benefit rather than a hindrance in the case of the Arab Spring in Egypt (Faris, 2013). In that situation, even though most people who had access to the Internet were elites, these elites used their access to engage online, and extended their digital knowledge to those offline who had little or no access. Many of those who were digitally active were affluent college youth. They organized the protests and connected with their counterparts in the streets through face-to-face meetings and interactions. Faris argues that their online engagement had an influence on the offline 
activism that happened in the streets during the protests. One of the characteristics of the Arab Spring and other popular movements was the use of data-enabled mobile phones: these are becoming an increasingly important part of public and communal activities (Faris, 2013; Gordon, 2002; Hampton, Goulet and Albanesius, 2015; Hatuka and Toch, 2016; Shao and Wang, 2016; Shim, You, Lee, and Go, 2015; Volkmer, 2014;). Mobile phones have become important tools of communication and information dissemination: they are strongly supported by Internet technology and have attracted an increasingly active audience (Faris, 2013; Gordon, 2002; Volkmer, 2014). While Gordon cautions against overestimating the importance of mobile phones as a tool of public sphere activity, she accepts the argument that the flexibility inherent in mobile phone technology makes them vital tools in crisis situations. Moreover, there is practicability, which a computer or tablet may not offer, in a mobile phone for connecting to a wider community when an individual is in transit.

For Volkmer, new communication forms - specifically the Internet and mobile devices have enabled new communication models that are reflective and reflexive, rather than 'logical', forms of communication (2014). These new communication models often feature new and enhanced content, including reader comments and commentaries, blogging, and journal entries. Mobile phones are no longer restricted to personal use, but are valuable tools for community organization, information dissemination, and wider socio-cultural and political processes of engagement. While a person is away from a computer terminal, or when a laptop is shut off or tablet powered down, a person can still be connected to a networked community through a mobile phone. The advanced digital functionality built 
into new mobile phones means that they are no longer restricted to personal use; for instance, mobile phones were used 'subversively' in the Philippines in 2001 to oust the President, an historical event Gordon argues can be considered as a Fiskean form of 'guerrilla warfare' (p. 19). After attempts to impeach Philippines' President Estrada for corruption and malpractices were compromised by his supporters, the citizens took action by organizing public meetings using text messages.

Gender and Public Sphere Activity

This section examines literature dealing with the position of women in the public sphere, and the strategies that these women employ in order to engage in democratic and public sphere processes. This often involves creating alternative routes of access to the public sphere: it refers to the utilization of women's gatherings as public sphere sites in and of themselves; and the use of mobile phones, Internet cafes, and private living spaces to facilitate participation in political and communal activities. The issues covered in the scholarship are as follows: gender, the public-private dichotomy and the digital divide; gender, politics and the public sphere; and alternative practices and spaces that have been and are being utilized for public sphere communication and discussion.

Carol Pateman's work is important for understanding how women have been denied public sphere access because the discourses, rules, categories and logics of the civic and public spheres were strongly and more or less exclusively masculinist (Pateman, 1988). The discursive, socio-cultural and legal contexts and practices that bring about and normalize 
this exclusion are not always identified in political feminist writing about patriarchy. In other words, social contract, political and feminist theorists often engage the concept of patriarchy in their work without a distinctive critique of its role in gendering civil society. As Pateman writes: "The continuing domination of adult men over adult women disappears from view when patriarchy is reduced to the language and symbols of paternal (or perhaps parental) power over infants and children" (p. 22). Detaching the private from the public, without paying critical attention to how the public is constituted as a masculine space, normalizes, accentuates and intensifies gender inequality. This situation is rationalized by way of social contract theory, which presents male dominance of women as a neutral and natural event. By way of example, consider Graham's account of the lives of Brazilian women in the 19th century, which challenges the notion of woman as discrete, silent or indecisive (Graham, 2003). In these Brazilian women's cases, there was a clear distinction that was strictly maintained by patriarchal authorities and Christian institutions in 19th century Brazil between private and public spaces. For Graham "the two zones [of public and private] further correspond roughly and never exclusively, to male and female. Public street life was principally and comfortably a male domain ... [while] the interior quarters of the house were properly a woman's domain" (p. 29). This male/female and public/private dichotomy would later become more fluid and complex, in the process blurring the dividing lines distinguishing what constituted public or private life. Moreover, Brazilian laws inadvertently provided the opportunity for women to enter public life by granting them the same property rights as men. These property rights were not always observed in practice, but the three women in Graham's account took advantage of these laws to thematize private issues in public domain. Graham's account is useful for interrogating the problematic 
distinctions of public (street) and private (house) in modern societies. By extension, it is useful to refer to Abraham's (2010) critique of how an a priori definition of what is public versus private makes it difficult for those in a community to question the social construction of those categories.

Social inequalities can lead to a digital divide for women. This divide was present in the cases of Egypt and Tunisia during the Arab Spring. Faris makes the point that "women have played a significant role in the digital dissent that has swept the region [the Middle East] over the past ten years" (2013, p. 213). Egyptian digital activists play a similar role to the embedded e-activists I will discuss in a later section of this thesis. In the early 2000 s, Internet access was expensive in the Middle East, as it was in most other developing Southern regions of the world. Working class people competed for access at cyber cafes and via illegal sharepoints set up by unofficial third party vendors, who benefitted financially from their own individual dial-up access (Faris, 2013). Despite these challenges, women still managed to participate in the Arab Spring and make significant contributions to the protests. New media scholarship contributes to these examples, in order to draw attention to how social inequalities based on gender are being reproduced online. These examples demonstrate that the gender digital divide persists because of legal, political and socio-cultural reasons, rather than because women are less interested in technology than men (Foteinou, 2010).

Gender politics also strongly informs discourses and communication exchanges in virtual communities (Poster, 2001; Turkle, 1995). As we will see in the data that I have collected 
from WorldPulse.com, people are interested in knowing about the identity of the forum members with whom they engage. Why would this information be of interest if it were not to be used as a way of informing or guiding how people respond to other members of the platform? As most community members become more familiar with each other on the WorldPulse.com platform, they come to know personal information about each other, and such information influences discussions and interactions.

Looking at virtual communities that thrive on anonymous membership structures, the anonymity afforded community members in such communities perhaps influenced Poster's position on the constraints - or lack thereof - that gender poses in virtual communities. With a virtual community like WPc, which requires members' identity to be revealed, this situation does not apply. Indicating your 'true' identity is a major requirement on WPc: even if some of the members use pseudonyms, they often provide detailed information connecting their online persona to their offline life. Poster's argument is that virtual communities enable Internet users to construct and re-construct identities while engaging in deliberations in virtual communities and cyberspace as an arena for reconfigured political communication. However this misses the point that normalized gender discourses and politics are imported into and inform the communication practices of virtual communities, and that if these are reproduced and renormalized in online contexts and settings, it matters very little as to who is producing them: they will continue to circulate, and in this way they will help constitute the 'discursive world' in which people live, which will influence how they see and understand gender roles and meanings. 
It is apparent that people take on different personas depending on which platform they are using in virtual space. Sometimes within the same platform, someone can utilize and deploy multiple identities. Someone can be loving, caring and empathic on a discussion platform, while presenting as a fierce and competitive opponent in an online game. Turkle makes the point that for some people "experiences in virtual space compelled them to pay great attention to what they take for granted in the real" (1995, p. 256). She asks us to challenge the idea that there is a 'real' or 'true' self, and contends that there are multiple identities residing in the one person. For Turkle "the many manifestations of multiplicity in our culture, including the adoption of online personas, are contributing to a general reconsideration of traditional, unitary notions of identity" (p. 260). Turkle's position is that virtual identity is not fixed: it is potentially fluid, constantly in flux, and flexible and adaptable to different contexts. Turkle argues further that "[A protean self] is multiple but integrated. You can have a sense of self without being one self ... Home pages on the Web are one recent and dramatic illustration of new notions of identity as multiple yet coherent" (pp. 258-259). By way of example, someone writing in a journal about girl-child education might assume the role of a mother, but also take on the role of an education advocate within the same context. In the same space, that same individual can also be a site moderator or group leader, whose duties require her to be firm and dispassionate, while simultaneously representing herself as an independent concerned citizen.

But why do we have these changing notions of identity? And why should the ways they are manifested and utilized in online space be embraced as they are presented? It is expedient to ask why these multiple identities are seen - perhaps even considered necessary - for 
virtual space. What are the power dynamics, and the discursive and socio-economic regimes, that inform virtual space? Who benefit from people producing and re-producing themselves in their multiple and sometimes complex forms, while communicating and interacting with people in virtual communities? These are questions that we need to consider when looking at the changing notions of identity.

The status of gender politics in the public sphere is not necessarily static or fixed, just as there is an ongoing negotiation of masculinity and femininity in the private domestic practices of men and women. Cameron's (1998) case study of households in Australia indicates men and women constantly negotiate gender roles. She writes: "By representing the feminine and masculine as blurred and muddied, as each 'polluting' and 'infecting' the other, the idea of fixed and opposed gender groups becomes increasingly difficult to sustain" (p. 294). Cameron's study is a useful model for addressing the politics of difference among identity groups in the public sphere. Through information gathered from one-on-one interviews with women living in Melbourne, Cameron analyzed how men and women negotiated gender politics. Cameron identified what she terms "an ontology of discordance", in which people acknowledged that they did not utilize or rely upon a fixed identity (p. 304).

Gring-Pemble's (1998) critique of one-way letter writing practices between Antoinette Brown Blackwell and Lucy Stone - two $19^{\text {th }}$ century American women - examines how they influenced the early feminist movement, and by extension an understanding of the significance of the public-private dichotomy. The exchange of correspondence between the women was a precursor to the establishment of what would later be known as the woman's 
right movement: "Not entirely public nor completely private, Brown's letters to Stone function as a site for transferring her private thoughts into public action" (p. 42). GringPemble's study is particularly relevant to understanding how WorldPulse.com can be evaluated in terms of how it functions for, and is used by, its members. WPc claims to be a global gathering place for women. However in terms of the ability of WPc to function as a public sphere, there are spaces where women first raise issues of common concern - for example, through their journal writing or by commenting on other people's blog posts. These practices are forms of electronic correspondence that can be likened to the ones exchanged between Stone and Brown Blackwell in the $19^{\text {th }}$ century.

Social Movements and the Public Sphere

This section discusses scholarship that is concerned with the politics of the public sphere, and the relation between more conventional and alternative public sphere sites and spaces. Some of these spaces include the community websites, blogs and social network sites assessed by Albrecht (2006); Bakardjieva (2011); Birchall (2016); Bolognani (2010); Edwards (2015); Etling, Kelly, Faris and Palfrey (2010); Gulyas (2015); Harte (2014); Houghton (2012); Prince (2004); Townsend (2015); and McKenna and Edwards (2016). Specifically, the literature covers social movements and social service organizations in the public sphere, and the use of old and new media for political and social activism. Tully (2013) observes that:

Over the last two hundred years, feminists, advocates of the rights of the 
child, workers demanding a say in the workplace, abolitionists, antiimperialists, and so on have employed this sense to make public and democratize relationships within the liberal institutional private sphere, often without any official right to do so and at great cost to themselves (p. 186)

In this context, Tully was discussing how the private versus public work on and over each other in the context of social activist movements and the task of constantly questioning power structures. Arguably, the media were influential in helping to achieve the goals of the pro-democracy movements during the 2007 anti-emergency rallies and protests in Pakistan. However, Bolognani (2010) warns that making a direct cause and effect linkage between the success of the protests and the use of the media is difficult to demonstrate. Bolognani conducted a case study of the pro-democracy and pro-judiciary protests that happened after Pervez Musharraf declared a state of emergency in 2007. He suggests that both old and new media were utilized in the protest, although there was a complex relationship between class divisions, issues of media practices and consumption, and the production of media messages for an international audience. The anti-emergency protests gained traction partly because the local Pakistani media, and social media savvy students, were at the forefront of the campaigns. Bolognani writes that "The original inequalities of Pakistani society were likely to be reproduced in the anti-emergency management of new media” (p. 407). The protests against the Emergency rule were articulated along class, economic and educational lines. No communal unitary voice emerged to rally the locals, because most of the media coverage was designed to draw international attention to the 
issues; hence the protesters, mostly students from the Lahore University of Management Services who used YouTube and CNN's i-Report platforms, employed discourses of democracy and human rights that they hoped would get the international media's attention and sympathy.

Social activists appear to utilize both old and new media circumstantially, while established social organizations purposely engage media technology for promoting their causes (Bakardjieva, 2011; Prince, 2004). In a study of voluntary organizations in Australia, Prince (2004) found that the six organizations in his case study were aware of, and purposefully sought out opportunities to engage with, the media in disseminating information about their organization's work. The organizations used celebrities, entertainment or edutainment to reach certain audience demographics, ones that would otherwise tune out direct messages coming from voluntary organizations seeking financial or human resource support. The person-to-person appeal - which was augmented by the celebrity influence - aided in bridging the gap between the organizations and their target audience. These campaigns are run on social media platforms, and hence still fed into the larger public sphere.

Prince argues that "the lines between news and entertainment have blurred" (p. 149). This is a problem that Habermas foresaw when he first wrote about the public sphere: he pointed out that the media seem to have co-opted the public sphere and turned it into a space for commodity consumption, rather than a vehicle for engaging in rational-critical debates. The ways and means organizations communicate with their audiences have changed 
significantly over the last two decades. These changes were influenced by advancements in computer mediated communication that have inbuilt audience feedback, for example involving email and listserv. For Prince "new information technologies could lead to the strengthening of a certain kind of public sphere" (p. 155). The voluntary organizations referred to the use of these technologies in ways that they would not have been able to access or use TV or radio for similar fundraising or community campaigns.

In another case, there were some noticeable changes in the Bulgarian public sphere after a civic protest in the summer of 2007. There is a correlation between the civic protest - which was widely reported by bloggers - and the decision to debate the cause of the civic protest in the Bulgarian parliament. Bakardjieva makes the point that "[Bulgarian] civil society has been chronically weak both before and after the fall of communism" (2011, p. 64). She shares Silverstone's (2007) position that envisions a combination of old and new media in helping to produce an effective public sphere, one in which plurality and diversity would foster access to decision makers, while also presenting opportunities for policy changes. Silverstone's concept of the mediapolis proposes that combining new and old media is a prerequisite for Internet technology to be fully useful in civic engagement. However, she maintains that the vertical broadcast feature of TV and radio augment the Internet. Her case study shows how the combined use of online and traditional media was instrumental in the restoration of the natural reserve status for Strandja Mountain in Bulgaria. Less formal individual Internet bloggers' posts and forum participants' comments were interlinked with the more formal and structured publications of news in hardcopy newspapers, and broadcast on TV stations. The traditional media also connected their news coverage to their 
own websites, as well as the blogs of individuals, including hosting the bloggers on newscast interviews, as the protests continued, with eventual success, on the streets.

Non-governmental organizations using their networked resources, especially those with a focus on environmental issues, have played a prominent leadership role in monitoring and publishing information about the judicial proceedings in the Bulgarian case. Bakardjieva writes that "as places of visibility in the mediapolis, NGO websites have a specific and significant contribution to make" in civic engagement and in helping to promote accountability in government (2011, p. 76). WPc also makes use of this feature of combining traditional and electronic media in its digital action campaigns. One of its advantages in channeling women's voices is that it has secured opportunities for its members to be included in debates about global issues occurring on mainstream media like $\mathrm{CNN}$ and the Huffington Post.

There is a general acceptance among scholars that the Internet is a viable avenue for deliberating on political issues (Dahlgren, 2005, 2012; Papacharissi, 2002; Poster, 2001). Despite the fact that deliberation is the most difficult aspect of political practices to assess online, there is growing interest in the use of the Internet for deliberating on political issues among citizens. In addition to deliberation about political issues, information provision and voting are two other major political practices that are gradually embracing the use of digital technology. Albrecht's (2006) article is a mixed methods research project that studied a group of volunteers in Hamburg, who were recruited to participate in a political debate on a web-based forum that was organized offline. However his study suggests that "access to the 
Internet is not distributed equally but follows well-known factors of inequality, such as income, education, gender, age, and race" (p. 64). Albrecht's observation is similar to the outcome of Alzouma's (2013) report on the digital divide observed among citizens in Niger.

How might the Internet function as a tool for advancing political deliberations in a democratic society? It is expedient to know who participates and who is represented - that is, which voices are heard - in web-based forums. Etling et al (2010) consider public sphere practices in Arabic-speaking countries by examining blogs and bloggers operating on national lines. Bloggers in some of these Arabic-speaking countries focused mainly on local political issues, an example being how Palestine serves to 'unite' the entire Arab blogging network. Finally, Houghton suggests that the 2009 New Zealand Internet blackout was effective as a counter-publicity and counter-hegemonic force (2012). He argues that the protest was influential with regard to some of the policy changes that would later be introduced by the New Zealand government about Internet technology.

The Global Public Sphere

There are ongoing debates about the potential for a universal global public sphere featuring communal agendas that transcend nation-state interests. Fraser (2007); Poster (2001); Ugarteche (2007); and Volkmer $(2007,2014)$ have made significant contributions to this debate. Some of the issues arising for these scholars include the practice of nations prioritizing their interests over global agendas; the influence of language on globalization agenda; and Volkmer's proposal that discursive spheres in the digital age can be assessed as 
public horizons rather than public spheres. Contrary to Fraser's (2007) position that nationstates are placing less emphasis on national interests and working more on global transnational agenda, Ugarteche suggests that "the Westphalian state is alive and well" (2007, p. 66). Poster (2001) agrees with Ugarteche's sentiments about nation-states still privileging their own interests over any overriding global agenda.

National governments still make policies and rules of law along lines of national and state interest, as opposed to necessarily being dominated by globalist discourses emanating from strong Western democracies such as the United States and Britain. Ugarteche observes that "the concepts of the 'post-national', of 'Empire' versus 'Imperialism', seem to assume there are no national interests and that no armies lie behind these interests" (p. 66). Ugarteche suggests that "if public opinion is meant to affect the laws of the people who are governed, this remains very much national and remains more than ever somewhat ignored" (p. 67) in the macro public sphere. In Canada for instance, until the liberal government of Justin Trudeau took over from Stephen Harper's conservative government in 2015, the country refused to ratify any of the Kyoto agreement on greenhouse gas emissions, global warming and climate change. Other countries have also been very selective in signing on to global agreements through international governing organizations such as the UN, IMF and the ICC.

Language and trans-nationality are interconnected in the discourse of communication practices in the public sphere. Warner argues that public discourse is not the same as conversations. Language functions in discourse as a constitutive apparatus that enables a 
public to take certain shape or form. For Warner "merely paying attention can be enough to make you a member [of a public]” (2002, p. 415). The rational-critical discussion Habermas idealizes as a key requirement for the public sphere presumes that a particular public already exists outside of language or discourse. Warner challenges this Habermas ideal, and argues that a public is constituted by discourse.

Although English may be the lingua franca in the academic field and for business activities, there are other languages - Spanish, German, French, and Chinese - that tend to dominate debates about the global public's reform agenda. Ugarteche writes that "in an age of increasing intolerance and militarization of international affairs ...to speak of the postnational seems as eerie as referring to the death of imperialism. Power is 'national', has a flag, an army and interests" (p. 66). However, other scholars have argued that in spite of globalization and the influence of external states on internal politics and policies, the nation state can still make some autonomous decisions (Poster, 2001).

Over the last two decades, Internet technology and advancements in satellite transmission capacity have fast tracked the possibility of the notion of the global village as envisioned by Canadian communication theorist Marshall McLuhan, and what Volkmer (2007) identifies as the global public space. Volkmer focuses on news broadcast stations in arguing that less dominant non-Western media organizations, such as MBC and ZEE-TV, have managed to engage in political discourses in the global public space as much as their Western counterparts such as $\mathrm{CNN}$ and $\mathrm{BBC}$. This development is crucial to identifying and acknowledging the multiplicity that characterizes the space, rather than theorizing this 
relationship as dichotomous positioning that pitches 'Western' against 'Moslem' media (Volkmer, 2007, p. 56). She suggests that:

In addition, a variety of small 'grassroots' stations have emerged. They tend to be supported by organizations, such as NGOs and even individual entrepreneurs and, although serving 'only' transnational micro audiences, they provide authentic and powerful news 'flows' within this globalized infrastructure (p. 64).

Volkmer proposes the notion of public horizons rather than public sphere, because of the concept of the 'civic self' (2014, p. 181). For Volkmer "spheres of connected discursiveness of 'public horizons' are increasingly relevant as communicative forms and communicative engagement and are no longer necessarily related to national cultures" ( $p$. 182). Volkmer's (2014) study participants indicated that international issues carry as much weight as national issues. The transnational study has participants from different regions of the world. Issues and events take on global consequences and people engage with them at transnational levels because network technologies allow solidarity beyond borders. Recent examples include the three terrorist attacks in France in 2015-16, the Belgium airport attacks in 2016, the attempted coup in Turkey in 2016, and the lone-wolf terrorist attack in Munich, Germany in 2016. Facebook users around the world engaged with these issues by activating the flags of the countries as their Facebook profile pictures. This practice can be likened to solidarities countries show when their nation is in distress by flying flags at halfmast, or other countries displaying the flags of those nations on public buildings as a display of support when allies are in distress. 
Conclusion

In conclusion, we can say that the relevant literature is equivocal with regard to the relationship between new media technology, globalist agendas and discourses, and the role and status of the public sphere. However it is clear that access to the Internet is not universal, and that the issues of social and digital inequality and the digital divide continue to disadvantage marginalized groups. Scholars caution that mere citizen engagement online cannot automatically fix whatever is broken in most countries' political systems; for instance, online deliberation is a challenging process that requires moderation and predetermined rules of engagement. These features have to be negotiated in a manner that will limit the possibility of exclusionary practices, although the potential for limiting access to discursive spaces cannot be completely ruled out if the notion of civility, as advanced by Habermas, continues to be an essential feature of political deliberation.

There is a great deal of recent scholarship which takes the position that public sphere theory in its revised form as communicative action is still relevant in media studies, and is useful for assessing the communicative and deliberative practices occurring in virtual public spheres. However at the same time it is clear that there is a significant body of scholarship calling for a revision of how the public sphere, and public sphere activity, is defined and understood; and that this body of scholarship considers Habermas' theories about the public sphere to be limited and problematical. Traditional media's hitherto unique power to set agenda and influence topics of debate in the public sphere has been diluted by the 
proliferation of social media platforms like Facebook and Twitter.

The gender and public sphere scholarship acknowledge the intersection of gender identity, politics, new media and access to the public sphere. The social media and public sphere scholarship suggests that there is a distinct difference in the ways that established social service organizations use new media in contrast to emergent social movements. National interests are still privileged over global agendas, and hence language has an influence in determining what issues are prioritized in the global public sphere. There is an obvious gap in the literature about the intersection of gender, offline-online activism, and civic engagement in the public sphere. I anticipate that my research can help to fill this gap through its critical assessment of the work being done by the members of worldpulse.com in the digital action campaigns and in the citizen journalism 'Voices of Our Future' training program.

In order to undertake this research, I have chosen to make use of a variety of complementary research methodologies, and the following chapter will describe and justify these choices. The methodological mix is informed by a desire to focus on the dialectical potential of new media technologies and social science theories and methods, rather than the persistent dichotomous positioning of both the theories and practices. As part of this account, the chapter will also discuss my volunteer work as a 'Voices of Our Future' listener and 'Digital Action Campaign' commentator and assessor, while I was a participant-observer on the website. The volunteer work gave me a glimpse into and understanding of the culture that characterizes, and the work carried out by, 
WorldPulse.com. 
Chapter Three: Methodology

Introduction

This chapter will explain and justify the methodology used in the upcoming discursive analysis of worldpulse.com and its activities. The methodology used in this thesis is best characterized as a mix of discourse and content analysis and virtual ethnography based on extended case studies, and interviews with participants. The discursive analysis will predominantly target the website and the texts and markers of what I have termed its culture: this includes everything from more formal and articulated rules and requirements, to 'unwritten' but influential protocols of and for communication.

The virtual ethnography methodology makes use of extended case study in order to provide a useful and useable account and set of data of the website culture 'in action'; in other words, the idea is to identify and produce material that demonstrates, in both qualitative and quantitative forms, the 'logics of practice' that underpin communication on the site. This is important because the object of this study is to determine to what extent, and in what ways, communicative action on the website can be understood as a form of public sphere activity. Identifying and producing material that allows us to understand the logics of practice that determine communication protocols have two main functions with regard to the issue of whether the site constitutes a legitimate public sphere space. Firstly, it allows us to compare this culture to the various sets of Habermasian criteria of the public sphere that are still influential, although subject to constant and widespread revision, in scholarly literature. 
Secondly, if these criteria are not close to or commensurate with the culture on the website, but we can still identify sets of discursive practices on the website that can be characterized as critiques or discussions of socio-cultural and political issues, then we need to reconsider the extent to which Habermasian public sphere criteria are still reliable indicators of, or criteria for determining or evaluating, public sphere spaces and activities. Flyvbjerg (2004) makes the point that "case studies often contain a substantial element of narrative. Good narratives typically approach the complexities and contradictions of real life" (pp. 429-30).

My aim in the methodology was to explore multiple dimensions of the research questions by not just identifying and evaluating communication practices, but also by asking participants to reflect upon and talk about their practices. The idea was to triangulate the areas of discourse analysis, ethnographic and contextual analysis, and interview data. The purpose of this kind of triangulation "is to enable researchers to plan and examine critically the logic, composition, and protocols of research methods; to evaluate the performance of individual techniques; and to estimate the likelihood of particular research designs to contribute to knowledge" (Krippendorff, 2004, p. xxi). In this context, I conducted a multistage data triangulation of information, which was largely obtained through discourse and content analyses, and a web survey. The discourse and content analyses and the web survey were augmented by follow-up semi-structured email interviews and a researcher's journal that I kept during the project.

In employing this multimodal epistemology, I used Habermas' theory of the public sphere as the departure point for evaluating and analyzing the data. In situating Habermas' theory 
within the context of the digital public sphere, I then employed DiMaggio and Hargittai's digital inequality model to assess how the normative requirements of public sphere theory are problematized when assessed in a digital domain. Foucault's concepts of discourse and language were also used in thinking through some of the complexities I observed in the data. In the course of coding the data obtained from WPc, some emergent codes were added to the a priori codes. I observed that there were some information in the data that were not found in Habermas' and DiMaggio and Hargittai's theories. In the second stage of data analysis, I then assessed these emergent codes as a group on their own; in analyzing the data, I identified a pattern that resulted in the development of the civic engagement typology. The civic engagement typology was derived from an analysis of the themes and issues that were identified in the data. The data and participants for this research were drawn from the forum WorldPulse.com. The analysis focused on the following areas: discussions about political and global issues; the extent to which the voices of the members were represented in the debates about issues of global concern; how members access the online discussion forum; and the value they place on their contributions to debates on the website in relation to discussions of economic and socio-political issues in wider global communities.

In the first phase of the data collection, I conducted a content analysis of the journals and comment postings on WPc. I have already referred to the way in which discourse analysis was employed here: basically the point of discourse analysis is to determine the extent to which, in Foucault's terms, language produces the realities that it purports to be describing (Foucault, 1995). Along with the discourse analysis, I also made use of content analysis. 
Content analysis "entails a systematic reading of a body of texts, images, and symbolic matter" (Krippendorff, 2004, p. 3). The content analysis was followed by an online web survey of the members of the worldpulse.com community. Web surveys "are attractive because they have several advantages. They allow for simple, fast, and cheap access to large groups of potential respondents" (Bethlehem and Biffignandi, 2011, p. 2). The web survey was particularly useful for helping to understand why members use the WPc forum, and what kind of work they do on the forum. The third phase of my data collection involved semi-structured email interviews of WPc community members and the website's management team; this enabled me to better assess and contextualize the information that was obtained. This multimodal approach employed in the research help to produce a detailed account of the discourses on the WPc website. The data collection, assessment and coding strategy are further outlined below.

Virtual Ethnography

Ethnography is a form of research that requires researchers to be immersed in the cultures and communities they are studying. The researcher is positioned as a participant-observer, who is involved in the phenomenon being studied while also observing and assessing the practices of the subject(s) of research. Hine advises that:

In its basic form ethnography consists of a researcher spending an extended period of time immersed in a field setting, taking account of the relationships, activities and understandings of those in the setting and participating in those 
processes $(2000$, p. 4).

The advent of the Internet has meant that ethnographic study has now been extended to the digital domain, such that a researcher can become immersed in cyberspace (including gaming communities, dating websites, political forums, social media sites) while assessing and analyzing the practices of the people using these cyber platforms. Hine (2000) writes that:

There is a place for a study of the everyday practices around the Internet, as a means to question the assumptions inherent in the prediction of radically different futures. Ethnography is an ideal methodological starting point for such a study. It can be used to explore the complex links between the claims which are made for the new technologies in different arenas: the home, the workplace, the mass media and the academic journal and monograph. An ethnography of the Internet can look in detail at the ways in which the technology is experienced in use (Hine, 2000, p. 4).

There are three central elements to virtual ethnography: space and time; identity and authenticity; and ethics (Steinmetz, 2012). Space requires that a researcher defines the boundaries of the field site: is it the entire website or sections of the website? My research project focused on the Digital Action Campaigns, 'Voices of Our Future' citizen journalism training program and back-end management resources sections of WPc. For Steinmetz, "In virtual ethnography, physical location does not dictate the object of study, but connectivity or interaction does" (p. 28). WPc can and should be defined as the extent of its intertextual 
practices; in other words, the website and the identities associated with it can be understood as a set of sites, what Bakhtin (1986) in Speech Genres \& Other Essays calls statements that have been engendered by an initial space; in this case electronic institution or identity and textual intervention.

However, there are activities outside the site that I consider relevant to my research as well. What the site owners and participants do post-campaigns is essential to understanding the effectiveness of the digital action campaigns; this includes the offline action they take in their various communities to redress issues they have identified and addressed, and postdigital action campaign activities, such as making presentations to third party organizations (for instance the UN and G8). I have delineated the parameters of the site in the context of electronic textual identities and practices by focusing first on the online activities, and then extending this to the events that happen offline. I use discourse extensively as a marker of ethnographic boundaries - "interactions of interest" - (Steinmetz, 2012, p. 28), using text to show boundaries, but also using it to interrogate boundaries and how these boundaries are shifting and being redefined with the increase in virtual activism.

Specifically, this study of WPc is a "virtual ethnography of message boards" (Steinmetz, 2012, p. 28). The three stages of this ethnographic study are: primary ethnographic experience, through my participation as a community member and community listener; secondary discourse and content analysis, which involved collating and reviewing archived journal writings already produced by participants on the site; and an online web survey of participants of the digital action campaigns and 'Voices of Our Future' citizen journalism 
training program. One of the more significant and problematical methodological issues confronting this kind of ethnographic work is the relationship between online and offline spaces. Steinmetz argues that the location of the website user does matter, because they are not communicating or presenting themselves on the Internet with regard to their offline contexts, history, identity or cultural affiliations. For Steinmetz, users are situated within, and inflected by, an offline context that can and will influence their online behaviour. He notes that "the different locations where each person was situated changed what topics they considered to be important and their perspectives on the matter" (Steinmetz, 2012, p. 29). Steinmetz suggests that "offline interviews can be used to supplement this online area" (p. 30 ), and points out that online and offline spaces are overlapping fields of activity with sometimes different but not necessarily non-continuous rationales, identities, logics and discourses.

Electronic sites (online) are derived from, and are subsets of, the physical (offline) sociocultural field; by way of example, they are informed by, and employ and recognize, similar forms of discourse and cultural capital. However, the online world constitutes a refracted version of the offline world, to some extent. One of the challenges in this project is to show how, and in what ways, these refractions occur. Ultimately the online world is still inflected by the field of power and the habitus (Bourdieu, 2000), but the defining characteristic of the online site is to differentiate itself from the offline, at least discursively; in other words, online identity is often predicated on the negative "I am not that" ('that' being one's online identity, and its socio-cultural restrictions), while (more or less necessarily) reproducing 'that' (cultural literacies, dispositions, discourses, the habitus) in many respects. Online 
identity is strongly inflected by this disavowal of the offline world, at least in the ways in which it sets out (largely utopian) claims for the online world and its communities.

However, every online identity is derived from, carries traces of, and discursively performs, recognizable offline identities and their associated discursive performances. It is interesting to look at how these wider utopian discourses inform individual contributions and forms of self-presentation. Every online identity will perform and present in a manner that is discursively recognizable from the offline world; it is how this takes place that is of particular interest to this project.

Steinmetz claims that "identity is flimsy on the Internet because it is easier to play with than in real life" (p. 31). Zizek also argues that there is less at stake in online identity (1997). Both of these assertions are difficult to argue with: while the question of what is at stake in online identity is a complex and complicated matter, what is undeniable is that one of the features of online social and communicative action is the difficulty of ascertaining the extent to which the performance of identity online corresponds to offline realities. At a basic level, this is because there is no obvious 'objective correlative' - a body, for instance that comes with an utterance or action. There are, of course, markers of identity in what Judith Butler would call online 'performatives' (Butler in Gender Trouble, 1990): in the offline world each subject embodies various sets of discursive performances (everything from spoken language to bodily hexis) that are commensurate with and 'give up' specific forms of identity (based, for instance, on gender, class, age, and breadth and type of cultural literacy). This is the basis of how we can analyze and make sense of the relation between online and offline identities and performances. 
For Turkle, gender identity occurs at the site of discourse in virtual communities (1995). Steinmetz also notes that "identity is generally not an issue" on the Internet, although he recognizes this to be a challenge for ethnographers (p. 31). Steinmetz advises that one should "focus on how identity is negotiated within this new environment by studying: 1) the digitally projected identities of participants, and 2) how the identities of participants are negotiated and judged as being authentic in and of themselves" (p. 31). However, what is at stake here is not just authenticity, but also which performances are recognized and count as authentic, and who decides what is authentic and how it is decided.

I addressed the challenge of identity verification by connecting online and offline personas through supplementary survey and interviews that are organized based on convenience sampling and selection. Arguably this connection of online and offline personas may produce more research problems; however, I used this connection sparingly and carefully. I then move into a more detailed discussion that is focused on discursive performatives and literacies, paying close attention to online communication cues such as emoticons, metamessages, acronyms, users' names, avatar, signature, and profiles. Specifically, I reviewed the profiles of the participants to select for phases two and three - online web survey and semi-structured email interviews. In this context, my focus is on identifying and analyzing the performance of identity.

In doing this kind of research, I dealt with a set of related ethical and methodological issues concerning anonymity, informed consent and age (Garcia et al, 2009; Murthy, 2011; 
Robinson and Schulz, 2010; Steinmetz, 2012). Steinmetz quotes Walford's contention that there is "no need to provide anonymity" (Steinmetz, 2012, p. 36). He further cites Sveningsson to the effect that "in gaining individual consent of each user in a bulletin board, we can consider their posts to be the same as open public letters/presentations which are not subject to consent" (Steinmetz, 2012, p. 34). I represented participants in the sense that I referenced their journal and comment postings in this research using their online identity as provided on WPc, both when I quote them directly and when I paraphrase their postings. Steinmetz makes the point that "Ideally, every thread the researcher observes and participates in should be preserved" (p. 36). I took screen captures of the journal and comments postings included in my data selection, and some of these are included as examples in the data report and analysis.

Institutional ethnography has developed techniques and theories based on and related to virtual ethnography. These techniques and theories are particularly relevant to my project, in terms of the online web survey of participants of WPc and the semi-structured email interviews of the community members and management team. DeVault and McCoy (2001) examined institutional ethnography, following the tradition of Smith's work, in order to analyze connections between everyday experiences and organizational control and power. Their article provides a literature review of the institutional ethnography field and also shows how to conduct institutional ethnography. They look at the works of institutional ethnography researchers who have been working in the field following Smith's model for the last 30 years: 
The researcher's purpose in an IE [institutional ethnography] investigation is not to generalize about the group of people interviewed, but to find and describe social processes that have generalizing effects. Thus interviewees located somewhat differently are understood to be subject, in various ways, to discursive and organizational processes that shape their activities (DeVault and McCoy, 2001, p. 193).

The authors outline the goals institutional ethnographers can pursue in their work, most particularly conducting research that explores the intersections and inter-connectedness in distinct institutional agencies, such as healthcare or education as institutions, to show how these relate to other operations carried out within them.

The use of interviews in institutional ethnography helps in analyzing the relations of power between people working in the institution under investigation; using healthcare as an example of an institution, the research could interview doctors, nurses, patients, and lab technicians to explore how they relate in their day-to-day interaction within the healthcare system of a certain city or community. The authors advise that the goals of institutional ethnographers often take critical perspectives. Institutional ethnography has also gained a strong following in feminist sociology and with feminist scholars.

DeVault and McCoy's article is a valuable guide for scholars who are interested in conducting institutional ethnographic studies. The authors outlined the interviewing processes used in institutional ethnography projects. They note that in institutional 
ethnography, interviewing is more about "talking with people" than it is about talking to them (p. 195): “The interviewer's goal is to elicit talk that will not only illuminate a particular circumstance but also point toward next steps in an ongoing, cumulative inquiry into translocal processes" (p. 193). Theirs is a continuum bordered on one end by planned interviews and on the other by "informal" interviews (p. 196). They note that institutional ethnography is more practical than it is theoretical in its application to phenomena. DeVault and $\mathrm{McCoy}$ also explore the processes institutional ethnographers use in analyzing and presenting their data; they conclude that institutional ethnography is useful for understanding everyday life and its most effective method is the interview in which a researcher talks with people.

This project is partly auto-ethnographic, given that I became familiar with some of the community members of WPc and the cultural practices of those posting journals and comments on the site. The strength of ethnography as a research methodology for my project is that it helped in obtaining information from participants of WPc at the site where they produce and reproduce themselves, and simultaneously assisted me in recording information about the environment, conditions, and cultural contexts in which these productions and reproductions take place. Conducting the study as a relatively 'distanced' and inactive member of WPc minimized any problems or issues that could have occurred due to my participant-observer status. As part of the process of conducting this virtual ethnography, I was a participant-observer on WPc. I volunteered to act as a listener in the 'Voices of Our Future 2013' (VOF) citizen journalism training program, and also in the 'Women Weave the Web 2014' (WWTW) digital action campaign. The journal entries and 
comments of WPc online community members during the digital action campaigns (DACs) were assessed using content analysis. I followed this up with an online web survey questionnaire that was open to all WPc community members but also targeted participants in the 'Voices of Our Future' citizen journalism training program. The survey produced data that show how participants evaluated the training and the extent it is commensurate with the claims made by WPc on its website. The procedural aspect of the data collection and analysis involved the following: obtaining ethics approval to conduct the survey and interviews; designing the survey and interview questions; piloting then administering the web survey; taking field notes while immersing myself in WPc's virtual environment; transcribing the field notes; conducting the interviews via email; identifying the categories of analysis, based on a priori codes and emergent themes from the data sets; coding the survey and interview data; analyzing the data; and writing the report.

Virtual ethnography is useful for assessing online communities with minimal potential of researcher's presence influencing the data or the outcome of the research, although this influence cannot be eliminated entirely in any social science research. There are risks associated with this type of research. These include the researcher being too close or too immersed in the community to be able to fully critique it; the researcher may miss insights into some of the experiences and ideas that should ideally be analyzed further; verification of online identity may be problematic; and the researcher's lurking status may affect the participation rate in data collection. However the benefits of virtual ethnography far outweigh its risks. Conducting virtual ethnography will opportune a researcher to obtain information from participants in their own environment; one can include diverse 
participants from different parts of the world; bias is minimized in that the researcher is not interacting with the participants face-to-face; and data is already archived on the website and can be accessed as needed.

Case Studies

Lockyer (2006) outlines the requirements for conducting a mixed methods case study, particularly with regard to the debates around the usefulness of the method, and the issues, processes, challenges, and suggestions for preparing the final report. She examines data triangulation, methodological triangulation, and theoretical triangulation, and argues for the mixing of quantitative and qualitative methods as a means of enhancing data analysis. Lockyer observes that mixed methods research is informed by the principle of triangulation, which she defines as "the use of more than one research approach to investigate a particular question" (p. 42). She notes that one of the first instances of such work, which was conducted in 1959, was the multi-trait-multi-method matrix used by Campbell and Fiske.

I consider mixed methods valuable and useful for approaching research questions from multiple angles. For instance, survey is quantitative and provides data that could then be explored using statistical models and this data may reveal serendipitous information that would not otherwise be available through interviewing. Following up initial data from a survey with interviews produce qualitative data that allows a researcher to contextualize the initial information obtained from the survey. Most especially, the email interviews I conducted in this research project allowed me to include people who are located in different 
parts of the world. These are people I could not have reached otherwise. Hence, this study's model of mixed methods case study is a reflection of the many advantages of digital media that my research was assessing in WPc's work. In approaching the project using mixed methods, I was able to combine the information that was already produced and available in WPc journal archives (discourse and content analysis) with quantitative information about the WPc members (using web survey) and contextual information about WPc members' experiences and reflection (email interviews).

Lockyer suggests that "many authors have rejected the assumption that convergence of results from different research approaches can be interpreted as a sign of validity" (2006, p. 42). She also notes that "although there may be strong correlations between the results of differing tests, the possibility of error is maintained, as both research approaches could be problematic" (p. 42). What she is referring to here are concerns about verifiability and validity: I intend to address these issues through careful and rigorous use of sequential triangulation; the results from the content analysis will inform the focus of the web survey, as well as guide the semi-structured interviews to be conducted with WPc community members and website management team. This process should produce a convergence of results, and also add scope and breadth to the study. The strength of using mixed methods is that it will produce data from multiple sources: this data will, at the very least, indicate the extent of any positive correlation between the claims made by WPc and the actual experiences of WPc members. However, the use of more than one method in examining the material does not necessarily translate to the emergence of valid results. 
As part of her argument for using mixed methods, Lockyer suggests that a study or analysis of any text or data can benefit from mixed methodological approaches, mixed methods of analysis, or a combination of both of these. The third option is more relevant to the work I did in this project. Specifically, my thesis used two research strategies, identified by Denzin as data triangulation and theoretical triangulation (Lockyer, 2006). I used data and theoretical triangulation simultaneously in the data analysis to assess both political activism (via the Digital Action Campaigns' call to action) and interpersonal computer-mediated communication (via the 'Voices of Our Future' training program). Habermas, Foucault, DiMaggio and Hargittai, and the grounded theory that developed from the civic engagement typology, were used progressively and inter-relatedly to assess the multiple sets of data that I obtained from the archived journals, web survey and email interviews. The content analysis of the archived journals produced the kind of data that allowed a systematic overview of the digital action campaigns of WPc, and assisted in identifying types of postings made by the participants and the frequency of their postings (Bryman, Teevan and Bell, 2009) while the web survey and email interviews allowed for an extensive contextualization of the data from the archived journals.

Similar to Lockyer, Albrecht (2006) used data triangulation and theoretical triangulation in his case study to explore a research question about who participated and which voices were represented in a political web-based forum. My case study of WPc took a similar methodological approach to Albrecht's study. His research questions examine which factors "explain participation and representation in the online deliberation" of a group of users who had voluntarily signed up to participate in a real-world political debate using a 
web-based forum (p. 69). He warns against the generalization of results, since there are conflicting reports on the effectiveness of the Internet as a tool for fostering deliberations online.

Albrecht points out that the forums he studied had clear rules and regulations and were heavily monitored by professional moderators, whose presence was very evident in the data collected: the moderators contributed 12.4 percent of the postings. This raises the question of whether the results from his study would be replicated if they were applied to the study of a virtual forum such as WPc. By way of example, Albrecht notes that "flaming," a recurring and offensive element that sometimes deters participation in online forums, was absent from the forum he studied. This is evidence that the real-world offline nature of the forum created an environment where participants might have felt compelled to be respectful and polite, two factors that may have also hindered participants' expression and behaviour.

Methods

The methods of analysis used in this project are discourse analysis (which is woven throughout all the data sets and extends to a reflexive critique of the theories and data); content analysis (primarily of the archival journal writings and comments from the 20122014 digital action campaigns on WPc); online web survey (with a specific focus on the "Voices of Our Future" citizen journalism training participants and select digital action campaign participants); and email interviews (of World Pulse management staff, digital action campaign and "Voices of Our Future" training participants). 
Discourse Analysis

This project aims to demonstrate, through the case study of worldpulse.com, that there is no singular, homogeneous public sphere: the public sphere has to be considered in its plurality. The case study does this by offering a body of description, analysis and evaluation of the culture and practices on the website that can then be used to demonstrate that the site's culture and practices reflect some public sphere typologies. However WPc also in various ways reflect some distinctions that can be described, analyzed, evaluated, and the ramifications of these distinctions can be developed into an argument-as-conclusion that indicates that some of the criteria are necessary ways for determining public sphere sites and activities while some are not very thorough or reliable criteria for this purpose, but merely generally indicative. Therefore those criteria need to be developed or modified if they are to be of practical use, particularly in terms of the issues raised by Foucault and the issues consequent of electronic technology, which together in some ways complicate the criteria put forward by Habermas to the extent of making them rather inadequate and problematic for determining what constitutes public sphere sites and activities.

Taken together, the ideals proposed by Habermas can be construed as indicators rather than criteria. Furthermore, the critique of the criteria and the typologies that is derived from the analysis of the site and its practices and culture (through discourse analysis and content analysis) lead to the conclusion that it is unrealistic to put forward or rely upon one set of criteria to determine public sphere activity, but that there are many different forms of public 
sphere sites (and sets of activities), based on many factors (for instance, what the aims of the site are, generic and cultural features specific to each site, demographic specificities, levels and kinds of cultural literacy, and levels of technological access). Discourse analysis can aid in conducting a much more sophisticated account, evaluation and argument about the relation between the culture and practices identified and analyzed on the site, and how we can make sense of public sphere activity.

Discourse examines how language is employed, who can use certain language, and under what conditions. Foucault's discourse suggests that the terms used in naming an event, a group of people, or even a phenomenon influence what kinds of meaning or interpretation is generated (Foucault, 1990). Schirato, Danaher and Webb (2012) suggest that,

The practices, systems, spatial arrangements and forms of address that characterize an epistemic shift are made possible by, and produced through, a particular discursive regime that not only brings into being and naturalises a different world and world-view, but also different forms of subjectivity, and ways of seeing and feeling (p. 33).

In this context, discourse can be used to analyze and critique the representations of and by WPc community members on the forum while simultaneously assessing the public sphere criteria and the ways in which these criteria are complicated by digital forms and its inherent inequality. Discourse analysis allows us to consider why and how certain experiences are legitimized while others are discounted in public sphere deliberations; for 
instance why are certain issues thematized and how do those issues become a communal concern for those engaging on the platform? Possession of power reflects in discourse in that those who can name or label someone or something are often able to exert authority over that which they name or label. One can interrogate the control mechanisms embedded in Internet discussion forums using Foucault's concept of discourse because Internet forums are often employed by their owners to further ideological agendas. In Foucault's discussion of language and ways of knowing, he argues that what is considered real (true) is so bound up in power that it is difficult to access it outside of the language that structures discourse.

In using discourse, it is essential to analyze the relationship between power and knowledge, and the ways these two interact in the production of human experiences. People are part of the production and use of power in their daily lives and experiences. Although one can be coerced into submitting to powerful regimes, the person also benefits from these power relations by virtue of their participation in the products and outputs of those interactions. For instance, Foucault argues that, "power produces knowledge... there is no power relation without the correlative constitution of a field of knowledge, not any knowledge that does not presuppose and constitute at the same time power relations" (1995, p. 27).

Discourse is the link that allows power and knowledge to work together in that through the use of language between people, power relationships are established and enabled while knowledge is then produced and circulated from the experiences that occur in the course of those interactions. 
Foucault would argue that the discursive formations that constitute the public sphere are themselves bound by the conditions of the discourse used in framing the legitimacy (or lack thereof) of the issues on which they deliberate. Essentially, since Foucault argues that humans are always engaged in discourse and there are social limitations around certain discourses, it is utopian to presume that determining what issues to discuss will be free of some form of hegemonic struggle. Additionally, such determination of issues and their subsequent deliberation would also be exposed to the politics of language and speech because of inevitable hierarchical positioning, since some members of the group have more socio-political capital than other members. The ideals of equality and inclusivity would be hard to fulfill in toto although surface-level assessment may suggest that all parties have equal opportunities to contribute to the discussion.

For example, Foucault saw confession not as the regulation of a hidden self but as a powerful act of self-regulation through discourse and by extension, making of oneself accountable to social power. In this context, the journaling practices on WPc could also be construed as a form of confession in the Foucauldian sense. Foucault argues that through such discourses enabled by confessions, institutions of power were able to gain access to an individual's body without using physical force or verbal coercion. Reflecting on a practice such as journaling on WPc would help us further interrogate class and social relations, and how these relations are shaped by the discourses into which people are born and with which they choose (or not) to engage. There is multiplicity of discourses - it is not just a discourse about sex or repression but also about institutional power and control. Foucault points out that the discourse of sex was not about silencing but finding diverse ways, through 
institutionalized power, of talking about sex by injecting it into every discourse. In some way, sex became the (open) secret at that time.

The use of Foucault's discourse analysis in this project is focused specifically on his fourth cautionary prescription, which is tactical polyvalence of discourses. This prescription allows an examination of the multiplicity of discursive elements; discourses' double bind; and the ways in which silence and secrecy shelter power, for example homosexuality was subverting the language of power by using the same language to free itself from discourse. Individuals need to develop the capacity to think and do things differently in order for change to occur. Foucault argues that individuals are born into a material world, which is constructed by discourse, language, and power. He grounds his theory - and its application as an analytical tool - in social relations, not just discourse alone.

When ideas are devoid of their historical context, hegemony takes over, since the people are generally unaware of the contextual basis of the discourses with which they engage. Such expulsion of history is what enables the conventionalization of most otherwise ideological discourses as "common sense" (to borrow Gramsci's term). Codes function as means of signification in certain discourses because these codes are generally imbued with power and ideology. Foucault draws attention to such institutionalized power by questioning the political reasons and implications behind techniques of power and knowledge. He notes that there are limitations and parameters set around discourses at specific points in time. For instance, $17^{\text {th }}$ and $18^{\text {th }}$ century politics of language and speech promoted repression of desire by paradoxically endorsing and encouraging confession of 
desires under the illusion of the repression of sexuality. While the language used in discussing sex was refined and couched in scientific discourses, confession and penance increased. This ironic mix of repression and confession served those in authority at the time because it was a technique of power and knowledge that they used to access otherwise private lives of individuals in the society. A form of institutional power and control was then instituted for the benefit of the state. The tool of confession/repression made individuals accountable to authorities. This tool works in neo-liberal democracies by individualizing otherwise communal issues. In this context, the state shifts the responsibility for services it should provide to individuals and constructs some individuals as inadequate or irresponsible when in reality, these services have broader social and political contexts.

\section{Reflexivity and Critique}

Social analysis should begin with various questions that get a researcher thinking about ideas that can develop into the study of a social issue (Swift, Davies, Clarke and Czerny, 2004). Social analysis is born out of a person's inquisitiveness about things going on in the society and why certain events happen, for instance who controls the flow of trade or money. Social practices are informed and disposed by the ideas that characterize a society; these ideas are constructs that glue things and people together (Harvey, 2004). Although these constructs make the things seem like a whole piece that exists naturally, Harvey points out that these things are irreducible, complex, and problematic. These things can be examined in relation to other things or can be taken apart to look at their internal workings. 
Dialectical analysis equips a researcher to engage in examining such phenomenon. Harvey refers to the symbiotic relationship between a researcher and the researched, and suggests that their relationship should be that of "two active subjects" who work on and over each other, one influencing the other and vice versa (p. 130).

At a more specific socio-political level, Jones (2007) notes that issues about combating and eradicating global poverty often utilize moralistic discourse. From a review of the 1990 World Bank Report on poverty, Jones highlights the report's identification of key "determinants of poverty [as] access to income-earning opportunities and the capacity to respond" (p. 23). He also cites its insistence that knowledge of the structures of social relations is essential in understanding social phenomena, such as poverty, homelessness, and joblessness. Jones suggests that a researcher needs to combine theoretical knowledge and historical analysis in order to provide an adequate account of historical processes ( $\mathrm{p}$. 148). He argues that a realist approach to the study of poverty is more beneficial than the orthodox approach because it exposes the notion that the issue is historical rather than natural. The orthodox and empiricist analyses excuses social structures and capitalism; further, they are often ahistorical. Critical analysis, on the other hand, emphasizes the historicity of poverty, and exposes the societal and institutional factors that aid in sustaining poverty.

Foucault's notion of the relationship between reflexivity and critique constitutes an important part of my methodological approach. There is a tendency, particular in work emanating from Habermas and his followers, to represent Foucault's approach, and more 
generally that of so called 'postmodernism', as being antithetical to the tradition of critique derived from Kant and the Enlightenment. Foucault specifically refutes this position. In The Politics of Truth, he identifies the Enlightenment with the creation of a historically specific "reflexive, critical sensibility" (Schirato, Danaher and Webb, 2012, p. 177), and argues that the spread of the reason of state and the apparatuses of discipline and governmentality both give rise not just to an institutionalizing of the discourse of rationality as a mechanism to justify the encroachment of the state into everyday life, but also to:

What we could call the critical attitude. Facing them head on and as compensation, or rather, as both partner and adversary to the arts of governing, as ... a way of limiting these arts of governing ... transforming them, of finding a way to escape from them or, in any case, a way to displace them, with a basic distrust (Foucault, 2007, pp. 44-5).

Foucault's use of critique-as-reflexivity is extended and contextualized with regard to the relation between critical analysis and the academic field. This ties in with the work of Bourdieu, who makes the point that the academy (and in particular the field of science) produces the critical disposition as a necessary part of the habitus of the field, and it rewards the manifestation of critique with cultural capital (1989). So if we look at one example of this process, famously outlined in Kuhn's (1970) The Structure of Scientific Revolutions, we could show that Galileo's experiments both destabilized, and eventually replaced, a particular scientific paradigm (not to mention its association with, and support of, Catholic religious doctrine). However, while his thinking 'against the field' and its 
limitations may have initially brought him a degree of opprobrium, in the end this was transformed into (positive) cultural capital. The field recuperates these paradigmatic fields, according to Kuhn, by way of a discursive 'sleight-of-hand'. As Kuhn writes:

In the sciences ... if perpetual switches accompany paradigm changes, we may not expect scientists to attest to these changes directly. Looking at the moon, the convert to Copernicanism does not say, 'I used to see a planet, but now I see a satellite'. That locution would imply a sense in which the Ptolemaic system had once been correct. Instead, a convert to the new astronomy says, 'I once took the moon to be ... a planet, but I was mistaken'. That sort of statement does recur in the aftermath of scientific revolutions. If it ordinarily disguises a shift of scientific vision or some other mental transformation with the same effect, we may not expect direct testimony about that shift. Rather ... the scientist with a new paradigm sees differently from the way he had seen before (Kuhn, 1970, p. 114).

Bourdieu situates this critical disposition within the scientific and academic habitus: since thinking 'against the field' (or more precisely, against the doxa of a field), and in the process extending (or even transforming) the field, is a prerequisite for the accumulation of cultural capital, subjects are disposed to think 'reflexively'. So for Bourdieu, reflexivity is tied up with the question of cultural fields, because subjects (and any dispositions they have with regard to reflexivity) are produced by and through specific cultural fields. It is those fields that tend to encourage and reward a reflexive habitus through the institutionalizing of a 
reflexive disposition in mechanisms of training, dialogue, and critical evaluation. The level at which I was a participant-observer on WPc reflects thick participation because I am a registered member of the website; this involvement resulted in thick description and thick interpretations. While analyzing the data produced, I reflected on my position as an 'insider'. This is different from the situation of Bradley and Shroeder (2009), who conducted a covert ethnographic study of poker players. These reflections thread through the data analysis in the coming chapters.

Content Analysis and the Digital Action Campaigns

Krippendorff writes that "content analysis is a research technique for making replicable and valid inferences from texts (or other meaningful matter) to the contexts of their use" (2004, p. 18). I conducted a content analysis of postings on WPc in order to read, analyze, categorize and evaluate both the communication practices and the wider culture of the website. I used qualitative content analysis, which Bryman, Teevan and Bell advise that, "the processes through which the themes are extracted in a qualitative content analysis are often left implicit, although they are usually illustrated with quotations from the text being analyzed" (2009, p. 300). By way of example, Altheid refers to ethnographic content analysis (ECA) that seeks to revise the themes or categories being used in response to generated content:

ECA follows a recursive and reflexive movement between concept development-sampling-data, collection-data, coding-data, and analysis- 
interpretation. The aim is to be systematic and analytic but not rigid.

Categories and variables initially guide the study, but others are allowed and expected to emerge during the study, including an orientation to constant discovery and constant comparison of relevant situations, settings, styles, images, meanings, and nuances (in Bryman, Teevan and Bell, 2009, p. 300).

This method expands the scope of research to include outside forces at play that may alter, modify or inflect the research. Another type of qualitative content analysis employs hermenuetics; this treats interpretation as an epistemology, emphasizing the meaningful nature of people's participation in social and cultural life. The central assumption is that the analyst must seek meanings from the perspective of the author and "this entails considering the social and historical context within which the text was produced" (Bryman, Teevan and Bell, 2009, pp. 301-302).

The sample frame for my project is drawn from member journal and comment postings on WPc. The WPc online member directory indicates there are over 60,000 users from 190 countries registered on the website, although data supplied by World Pulse staff puts the registered membership number at 22,800 as of late 2015 . There is no indication of how many of these registered members are active participants on the site. In the first phase, I studied the journal and comment postings by participants in the Digital Action Campaigns from 2012-2014 and the 'Voices of Our Future' sections of WPc. These postings are usually in the forms of journal writing, blogging, and comments on threads that are started 
by participants on the website, as part of the larger 'Digital Action Campaigns' and 'Voices of Our Future' sessions initiated by the management of WPc. Random sampling technique was employed here to give the journal entries and comments of each member an equal opportunity to be included in the sample size for analysis. Using random sampling for selecting the posts assisted me in collecting data that yielded the most relevant and useful information for assessing the engagement of the participants with the issues they identified and discussed, both individually and collectively as a community.

The sample size is based on all participants of the 'Digital Action Campaigns' and 'Voices of Our Future' citizen journalism training program of WPc. I used random sampling to determine which member's postings to include in the sample size. The level of activity of each participant can be determined by clicking on their handle, which shows the frequency and volume of contributions made by the participants through their blog and the journal entries they submit on the threads within the 'Digital Action Campaigns'. I used the identifying tags created by WPc, which pull all posts related to specific campaigns and programs, to collate the postings that are made in connection with specific campaigns and programs, for example "VOF 2013" for the 'Voices of Our Future program' and "Youth", "Education and Training" for some of the 'Digital Action Campaigns'. The relevance of the contents of the postings made by a participant can be used in gauging the participants' openly accessible active engagement on the forum.

My sampling strategy was based on obtaining a representative view of DAC and VOF participants and their involvement in the forum based on their postings. I narrowed the 
timeframe to 2012-2014 to determine which data to analyze. I used purposive sampling to select the posts, by reviewing all the postings that fall into the 2012-2014 timeframe identified for the 'Digital Action Campaigns' and then choosing the three campaigns that had the most journal entry and comment submissions. I coded the data by reviewing the journal and comments posted by the participants with regard to the campaigns on WPc within the identified timeframe. The analytical categories serve as an additional guide in determining which posts to select and include in the final analysis. I focused my search on information that provided insights into the expectations of participants about the forum, level of participation, the limited demographic information supplied by the participants, which campaigns generated the most and or least comments, as well as the relevance of the comments to the initial content of the campaign. I identified the frequency of postings in relation to the number of postings selected for the final analysis.

Online Web Survey: the 'Voices of Our Future' Citizen Journalism Training Program

The reason for conducting a survey is primarily to obtain data that will give insight into the characteristics of the people participating on WPc; the views of the participants on the relevance of their engagement; how they access, and what challenges they face in accessing, the website; and how they evaluate their contribution in terms of strengthening the communal discourse presented by WPc. Dillman, Smyth and Christian (2008) write that "for more than 75 years, sample surveys have remained a remarkably useful and efficient tool for learning about people's opinions and behaviours" (p. 1). Information gathered through surveys can offer depth to a research question. Through the application of a survey 
questionnaire, one can gather data for quantitative analysis and also a qualitative textual analysis that is informed by insights such as why the forum participants make or do not make certain postings, who the forum participants are, how the forum participants perceive their roles on the WPc website, and the relevance of their forum participation to the political activities in their communities (Kirby, Greaves and Reid, 2006, pp. 127-133). I used a computer-assisted questionnaire accessible through Qualtrics software in this phase of the project. The questionnaire included open-ended questions, which provided additional information that lead to the collection of collateral material. This additional information was not originally designed into the research, but nonetheless the information provided supporting and valuable insights into the questions posed in my project. Additionally, I worked out the details of administering the questionnaire in the data gathering stage and also reviewed the return rate of the questionnaire regularly, in order to ensure that there was a relative level of representativeness of the population of WPc forum participants in the responses.

In using the 'Voices of Our Future' citizen journalism training program for this phase of the research project, I assessed the potential of citizen journalism as a tool of accessing the public sphere; specifically, I looked at the question of the extent to which citizen journalism fosters participatory democracy or facilitates access to the public sphere. My focus was on marginalized groups, particularly women, who may not have the technological access, technical knowledge or language skill, or may still feel shut out of the predominantly patriarchal media field. I considered in what ways the media weblogs function as effective participation tools for minority groups to access the public sphere; whether the training of 
these women by WPc aids in fostering a more participatory democracy; and why citizen journalism appeals to these women.

To prepare for this phase of the project, I contacted the management of WPc and obtained their approval to contact the participants of the website and ask them to complete a computer-administered online web survey. The survey was made available from February 25 to May 25, 2015. The survey collected data from the general members of WPc who have participated in the Digital Action Campaigns and participants of the 'Voice of Our Future' citizen journalism training program. When contacting WPc members via the WPc member inbox system to ask them to participate in the survey, I used purposive sampling to select the participants who have VOF experience from the larger WPc community. These participants were selected based on their level of participation in the DACs as reflected in their journal writings, the frequency and richness of their journal writings and comments, and in terms of their participation in the VOF training program.

The population surveyed was the full membership of WPc represented by a sample frame of those participating in the online web survey. Dillman et al. provide a relevant analogy: they write that "To estimate within 5 percentage points the preferences of 100 million U.S. voters, one needs only to survey 400 randomly selected voters" (Dillman, Smyth, and Christian, 2008, p. 1). The survey instrument was designed so that I was able to gather some broad demographic data, involving questions about age bracket, gender, income bracket, educational level and location. I could infer the status of the members from some of this information. The identity of the respondents was still protected, since some of the 
participants use pseudonyms, and they also did not have to provide their real names on the web survey. I was able to gather some qualitative data through the online web survey; most of the questions in the survey design had room for respondents to give additional comments as follow-up to the alphanumeric answers.

I used the Qualtrics software for designing and administering the online web survey. The survey was available through the Qualtrics platform used by Victoria University of Wellington. In addition to posting the survey link in sections of the WPc website for the general membership to use, I also invited selected WPc members who have participated in the VOF training to complete this survey by sending them private messages on the WPc platform. The categories of analysis used in the web survey were drawn from Habermas' public sphere and DiMaggio and Hargittai's five broad forms of digital inequality; I assessed the data gathered from the online web survey through an analysis of what Dahlberg (2001) terms the "six criteria of the public sphere" and what DiMaggio and Hargittai (2001) term the "five broad forms of inequality".

The six criteria of the public sphere are:

Autonomy from state and economic power. Discourse must be based on the concerns of citizens as a public rather than driven by the media of money and administrative power that facilitate the operations of the market and state.

Exchange and critique of criticizable moral-practical validity 
claims. Deliberation involves engaging in reciprocal critique of normative positions that are provided with reasons and thus are criticizable - are open to critique rather than dogmatically asserted. Reflexivity. Participants must critically examine their cultural values, assumptions, and interests, as well as the larger social context.

Ideal role taking. Participants must attempt to understand the argument from the other's perspective. This requires a commitment to an ongoing dialogue with difference in which interlocutors respectfully listen to each other.

Sincerity. Each participant must make a sincere effort to make known all information - including their true intentions, interests, needs, and desires - as relevant to the particular problem under consideration.

Discursive inclusion and equality. Every participant affected by the validity claims under consideration is equally entitled to introduce and question any assertion whatsoever. Inclusion can be limited by inequalities from outside of discourse - by formal or informal restrictions to access. It can also be limited by inequalities within discourse, where some dominate discourse and others struggle to get their voices heard (Dahlberg, 2001, p. 2).

The five broad forms of inequality are: 
Inequality in technical apparatus. How does inequality in the adequacy of hardware, software, and connections, limit the ways in which different kinds of users can employ the Internet? Inequality in autonomy of use. How much control do people exercise over their web use? Among persons with Internet access, are there significant intergroup differences in the extent to which that access is at home, as opposed to work, school, libraries, or other community access centres?

Inequality in skill. Looking at "Internet competence", the capacity to respond pragmatically and intuitively to challenges and opportunities in a manner that exploits the Internet's potential. Inequality in the availability of social support. Exploring the distribution and impact of at least three kinds of support: formal technical assistance from persons employed to provide it, technical assistance from friends and family members, and emotional reinforcements from friends and family. Variation in use. How do income, education, and other factors influence the purposes for which one uses the Internet? (DiMaggio and Hargittai, 2001, p. 8).

These categories of analysis from Dahlberg, and DiMaggio and Hargittai, were complemented by emergent codes that informed the development of the civic engagement 
typology.

Semi-Structured Interviews: WorldPulse.com Participants and Management

In opting to do follow-up semi-structured email interviews, I focused on three groups of WPc community members: 1) survey respondents who have indicated their willingness to participate in the interviews; 2) DAC participants who provided their contact information as part of their journal or comment postings during the three campaigns; and 3) World Pulse management staff. The interview data gave access to additional information that was useful for the extended qualitative analysis. When contacting WPc members via email to ask them to participate in the interviews, I employed purposive sampling to enable me focus on specific participants who potentially can give richer and more contextual information. These are WPc participants who have VOF training experience and who have also participated in most of the digital action campaigns. These participants were selected based on their level of participation in the DACs as reflected in their journal writings, the frequency and richness of their journal writings and comments, as well as their participation in the VOF training program. Some of these participants published their contact information publicly as signatures in their journal writings and comments, while other participants provided their email addresses as part of responses to the survey questions. I used this publicly available contact information and the contact information from the survey responses to invite them to participate in the interviews.

Kirby, Greaves and Reid (2006) write that: 
Interviews are a special form of interaction between people, the purpose of which is to elicit information by asking questions. Generally, though not always, the researchers are the ones with the questions and the participants are the ones with the experience and information of interest (p. 133).

McCarthy, Holland and Gillies (2003) examine the ways that interviews are analyzed by considering the "theoretical, epistemological, and methodological issues" in relation to multiple meanings and multiple realities (p. 2). Their project is focused on describing how they made use of the multiple perspectives that emerged from the 93 interviews they conducted. They used postmodernism and feminism theories in exploring individualized points of comparison, and also examined objectivists' and interpretationists' ways of analyzing multiple perspectives of the same phenomenon. Their study had five analytical categories and they were reflexive in identifying the judgments they made in data analysis.

I used oral history interview technique for gathering data from the management team of WPc. The oral history interview is a form of historical research that uses depth interviewing. In-depth interview is "the method of using the qualitative interview as the primary means by which the researcher gathers data for his or her study" (Babbie and Benaquisto, 2002, p. 334). Interviewing is best done by a researcher who pays attention to detail, is skilled in listening, acknowledges her position, and is open to encountering and making effective use of collateral material, as reflected in the case study: 
Depth interviewing involves asking open-ended questions, listening to and recording the answers, and then following up with additional relevant questions. On the surface this appears to require no more than knowing how to talk and listen. Beneath the surface, however, interviewing becomes an art and science requiring skill, sensitivity, concentration, interpersonal understanding, insight, mental acuity, and discipline (Babbie and Benaquisto, 2002, pp. 331-332).

The focus of the interview is historical. This type of interview technique has recently become more prominent in social science research. This oral history interview technique often provides a different and contrary response to information found in 'official' documents, such as is the case with the postings on WPc. The advantages of this technique are its flexibility, interactive nature, and effectiveness in pursuing issues in detail. A common disadvantage is its weak reliability, which is linked to the often-unreliable memory of participants; based on this weakness, the results cannot be generalized.

The oral history interview of WPc community members was conducted using email technology, which by extension means that a combination of oral history and email interview techniques were employed to collect this third set of data. The email interviews were conducted over 14 weeks from August to November 2015. Burns (2010), Meho (2006), and Murthy (2008) both conducted email interviews and attested to their usefulness for gathering initial and supplementary data. Meho provided detailed guidelines for social 
researchers conducting email interviews in qualitative research. In his discussion, he expands on the "cost and efficiency" as well as the "democratization and internationalization" benefits of email interviewing (pp. 1285-1288). Some of Meho's guidelines include how to invite interview participants, construction of the email interview questions and the initial invitation to interview participants, researcher reflexivity and selfdisclosure, ethical considerations, participant consent, assessing the quality of data and appropriateness of research methodology, and time management during the interviews.

Burns conducted email interviews as a follow-up after survey respondents provided their email addresses as part of the survey responses. He used one-to-one emails, similar to the ones used in this project. The practice of combining data gathering tools allowed him to test the value of triangulation of methods. Among other observations, he encountered language ambiguities with one of his participants. This language ambiguity is similar to my experience with one of my interview participants who has limitations using English language. Such ambiguity could have been addressed, perhaps by employing a translator, if the interviews were conducted face-to-face. Some of the advantages Burns identifies include the accessibility of the data produced, since data is already transcribed as it arrives in email responses. A challenge is the sometimes drawn-out length of the interviews due to the back and forth between participants and researcher. However, not needing to transcribe the interview responses closes the gap in time lost. Another disadvantage is that some potential participants may have declined to partake in the interviews because of limited writing skills. A key advantage specific to this project is that I was able to access interview participants representing multiple countries and regions of the world that would otherwise 
be too expensive to reach using face-to-face interviews. Despite these limitations, Burns argues that email exchanges constitute a valid form of data gathering.

Murthy situates the importance of email interviews within the larger context of digital ethnography that is enabled by advancements in technology. From his study, he considers blogs as having the potential to function as public spheres, but also notes that the "Internet is a space of power relations" (p. 840). His list of advantages of online questionnaires mirrors some of those identified by Burns (2010). A disadvantage identified by Murthy is that "the digital divide persists" despite the democratic potentials of some of the research techniques available in digital ethnography (p. 848). In spite of this disadvantage, Murthy argues that the combination of e-questionnaire and email interviews provided rich data for his research. I found the observation by Murthy to be true for this project as well. Combining online web survey with email interviews provided useful and relevant data.

Kirby, Greaves and Reid (2006) advise further that researchers should be both interviewer and respondent when conducting interviews. They argue that collaborative researchers should strive to work with and inform the community they are researching, and be mindful of democratizing the process. They emphasize the need for reflexivity on the part of the researcher: they argue that researchers need to account for themselves in the process of data collection and analysis. Bryman, Teevan and Bell (2009) have critiqued survey research that involves interviews. They argue that these types of research are asymmetrical; that is, the interviewer extracts information from interviewees and gives nothing back. The authors note that interviews, particularly standardized ones, create hierarchical relationships 
between interviewers and respondents. I address the issues these scholars identified by following the advice of Kirby et al. about the need to be reflexive regarding these activities and also opting to conduct semi-structured interviews that allowed opportunities for extensive follow-up with the interview participants.

\section{Research Design}

The research design was predicated on the 'affordability' and usefulness of dialectical analysis. I was particularly interested in the dialectical potential of mixing theories and methods based on my epistemological position that subscribes to hermeneutics, which treats interpretation as epistemology. Knowledge is contextual and delimited by power relations and the kinds of truths circulating in a person's socio-cultural space. This way of knowing readily lends itself to an exploratory outlook that allowed me to combine multiple lenses from different theorists while simultaneously utilizing multiple methods and categories of analysis. The usefulness and practicality of this approach to research is borne out in the development of the embedded e-activists' typology, which emerged organically in the course of assessing the multiple data sets using two pre-existing analytical categories that did not fully capture the information I observed in the data sets.

\section{Ethical Considerations}

In 2013, I emailed the WorldPulse.com management team asking if I could collaborate with them in conducting my research, and I received a reply asking me how they could assist 
with my project (see Appendix 15). In doing this, I anticipated that I could form a mutually beneficial relationship with the organization and conduct this research project with their approval, especially since I was conducting a virtual ethnographic study. This was also a way for me to make use of the collaborative opportunities Web 2.0 affords Internet forum members and social science researchers. I also expected that if need be, World Pulse management would share back-end data with me and assist me with additional resources, including setting up interviews, traveling with them on offline campaign meetings, and going on site visits to get first-hand in-person account about some outcomes of their digital action campaigns.

I then applied for ethics clearance and got approval from Victoria University of Wellington to conduct the research. My communication with WPc management formed part of the supporting documents I submitted with the ethics application. The ethical consideration for this research focused specifically on the data collection, storage, retrieval and use processes and practices. I shared the ethics clearance (see Appendix 16), which was obtained from Victoria University of Wellington, with the management of WPc for administering the online web survey and conducting the email interviews.

\section{Researcher's Journal}

A researcher's journal in critical cultural studies functions as a tool for documenting the reflections of a researcher and tracing the researcher's subjectivity and potential influence while conducting research. Ortlipp (2008) advises that "rather than attempting to control 
researcher values through method or by bracketing assumptions, the aim [of keeping a researcher's journal] is to consciously acknowledge those values" (p. 695). I started keeping a journal documenting my experiences as a participant-observer on WPc as a way to record my involvement in the community, and also to reflect on some of my observations as I engaged and interacted with WPc members and management. Ortlipp refers to keeping and using two reflective journals while conducting a doctoral research study about an early childhood practicum. She notes that "keeping and using reflective journals enabled me to make my experiences, opinions, thoughts, and feelings visible and an acknowledged part of the research design, data generation, analysis, and interpretation process" (Ortlipp, 2008, p. 703).

Janesick's (1999) article is written as a journal "to show how a journal may be used as a qualitative research technique" (p. 505). She explores journal writing during the research process that could be done by a researcher, and also journal writing that could be a collaborative effort involving researchers and research participants. Both types of journals produce data that can either be analyzed as part of the research instrument, or used in reflecting on the research process and the position of the researcher during the course of the research project. Janesick characterizes researchers' and participants' journals "as a powerful heuristic tool and research technique" (1999, p. 506). Janesick also notes that in the "use of a journal as an interactive tool of communication between the researcher and participants," journal writing gives a researcher the advantage of conducting interdisciplinary triangulation of data (p. 506). This potential for data triangulation is relevant to this research project as I reflect on my position while examining data produced 
during the three different phases of data collection in this research. As Watt writes:

Learning to reflect on your behaviour and thoughts, as well as on the phenomenon under study, creates a means for continuously becoming a better researcher. Becoming a better researcher captures the dynamic nature of the process. Conducting research, like teaching and other complex acts, can be improved; it cannot be mastered (Watt, 2007, p. $82)$.

Watt kept a journal for two studies about qualitative methodology; she used the journal to assess reflexivity in the first study, and "as a stimulus to reflect back on the original pilot study" when conducting the second study (Watt, 2007, p. 83). This is different from Janesick's practice, both as a researcher and also in terms of her collaborative work with research participants. I have noticed that my system of keeping a researcher's journal is similar to Ortlipp, Janesick and Watt, although I did not encounter their literature until after I started keeping my journal. While some of the data that I produced while keeping a journal thread through most of the chapters in this research, some specific reflections will be introduced and assessed in the data report and data analysis chapters.

Journaling on WorldPulse.com

The WPc website claims that it is "bringing women a global voice. We are using digital media to connect women worldwide into a powerful force for change" (WorldPulse, 2012). 
The members express their opinions by writing in journals and blogs, which are visible in the openly accessible areas of the website. WPc discursively produces itself as a social media site for women who wish to contribute to and help generate a collective voice, especially during its periodic digital action campaigns that focus on specific issues in diverse regions of the world. Some of the data I assessed were drawn from periodic campaigns conducted by WPc. By way of example, on the 27th of March 2013, WPc launched a six-month long campaign called 'Girls Transform the World Digital Action Campaign', with a specific focus on Central and South East Asia. WPc partnered with Asian University for Women in this campaign. Proceedings from the discussion and campaign on the WPc website were presented at the G20 meeting in Russia. Other recent examples include 'Take Action on International Women's Day' and 'Dispatches from the Global Struggle to End Violence Against Women'. A report on another previous campaign is accessible via 'Your Calls for Congo Peace Reached The White House'. Some of the issues identified by WPc community members during these campaigns would later form the subjects of their writings as VOF correspondents.

The deliberations on the website that occur during specific campaigns are presented at relevant international gatherings such as U.N. meetings or G8 and G20 proceedings. These presentations are sometimes led by the founder of the forum, Jensine Larsen, accompanied by WPc staff, selected members of the WPc forum and other prominent supporters of WPc. On a smaller scale, there are several measurable outcomes at individual and community levels; for instance, members report the progress they are making in their advocacy work in their various countries in the journals they post on the website. After presentations and 
lobbying, results of proceedings of the international meetings are assessed to see what policy changes have been effected in relation to some of the issues WorldPulse.com members addressed, highlighted or discussed during the campaigns.

WPc also trains some of its members to be citizen journalists through its 'Voices of Our Future' (VOF) initiative. In order to facilitate my research, I signed up to be a VOF listener in the 2013 competition. Listeners assess submissions by applicants who want to be selected for the VOF training. I attended two listener training calls via Skype on the $27^{\text {th }}$ and $29^{\text {th }}$ of March. Thirty successful applicants were selected to receive training, including individual matches with seasoned journalists who would mentor the trainees on how to be effective citizen journalists. About 200 applications were received in the first week, which was a low turnout compared to the $500-600$ applications that were processed in previous years. I received the first set of entries and assessed them between $9^{\text {th }}$ and $14^{\text {th }}$ of April. I assessed 23 applications over four weeks. During the process of assessment, I had questions around how 'fair' the volunteer listeners' assessments were, considering some of the listeners may not be knowledgeable about how to assess the VOF assignments, despite the best efforts of WPc to train them prior to the assignments being submitted for review. I also had concerns about what it would cost the listeners to access and assess these applications, especially the volunteer listeners. There are challenges of slow Internet connection and the finance needed to connect to the Internet, especially for those who may have to use computers in a cafe to do the assessment. It was long and tedious work; I spent an average of 28 minutes on each assignment - grading it, giving feedback both to the selection committee and directly to the applicants, and reviewing the materials attached to the 
applications for each week. Another concern I had was how one would be able to ascertain or verify that the applicants actually wrote the entries they submitted and that these entries were not written by third parties. However, this concern is not unique to this training program: it is a concern for both traditional and online classrooms. The foregoing observations are some of the issues that emerged from the data I collected on the website.

\section{Code Book}

For assessing the data produced, I developed a code book (see Appendix 19) to explain the terms that are used to code the data, and the values assigned to certain postings and why these values are assigned. The code book features a priori codes derived from Habermas, and DiMaggio and Hargittai as well as emergent codes derived from my observation of additional information while coding the data. The code book is a very crucial element: it was useful for ensuring that I was consistent in assigning value to the data and that there is a commonly agreed understanding of what certain things mean. I conducted a pilot test of the coding scheme to ensure there is congruity in the coding process before using the code book.

For the categories of analysis, a priori and emergent codes based on both inductive and deductive reasoning were applied to the data. The a priori categories of analysis are drawn from some of the discourses identified in the course of my involvement as participantobserver on the WPc website, as well as Habermas' six criteria of the public sphere and the five broad forms of inequality identified by DiMaggio and Hargittai. These discourses are 
combined with emergent codes that are identified from the online web survey data, followup semi-structured email interviews and researcher's journal. The emergent codes were then used to develop the civic engagement typology, which I will discuss in later chapters. I assessed the extent of the exchange on the forum by measuring them against the discourses of global voice, inclusivity, accessibility, representativeness, and changes in local and national policies provided by the WPc management on the website.

Conclusion

Virtual ethnography offers an opportunity for researchers to position themselves as participant-observers in the online communities that they study. This research was autoethnographic because I am also a member of the WPc community. Due to the nature of most websites having multiple sections and several layers of pages and subpages, it is important to specify the sections of the website that form the field of research to ensure that the enquiry is focused and in depth. For the WPc website, the focus was on the DACs and VoF sections. This virtual ethnographic research is a case study that used mixed methods in obtaining and analyzing data. Conducting a case study affords a researcher an opportunity to focus on a specific phenomenon within a micro context - usually practical and applied to an event, activity, incident or observation of one or a handful of organizations. Using multiple methods to assess a case involves triangulation of data in order to provide a more robust understanding of the phenomenon one is studying.

The three methods used for obtaining data from the WPc platform are: 1) discourse and 
content analysis for the archived journal writings and comments already produced on the website; 2) online web survey that was made available to the WPc membership through the Qualtrics software; and 3) email interviews of WPc members and management as a followup to gather additional contextual information for a more robust assessment of the data from the content analysis and online web survey. My researcher's journal was used as a memory extension tool throughout the research process to document events and activities about the research, including my involvement in the global \#bringbackourgirls campaign. Journaling on WPc mirrors some of the journaling practices that allow a reflexive approach to the discussion of issues identified on the platform. The citizen journalism training offered by WPc equips the website's members to be even more reflexive and professional in their journaling practices.

The code book used for assessing data prior to analysis contained a priori codes from Habermas' concept of the public sphere and DiMaggio and Hargittai's digital inequality model. In the course of coding data, emergent codes were added that later developed into a theme allowing me to propose the civic engagement model for embedded e-activists. This model is based on inductive reasoning. Ethics approval was obtained from Victoria University of Wellington after due consultation with WPc management, who granted their approval for me to communicate with their members for the purpose of this research.

The expectation of reflexivity and critique undergird the entire methodological process of this research from conception to completion. The opportunity to be self-reflexive and selfcritical was beneficial in the study of such a social phenomenon like WPc that has a strong 
feature of reflexivity and arguably does not attract sufficient critique. Political, social and cultural capital are constantly being produced and reproduced on the WPc platform with an expectation that these capital can be called on for individual or communal benefit when and if necessary. It is expedient to critically assess the reflexive practices - or lack thereof occurring on this platform.

The question of methodology and the effective selection and use of specific methods to research a social phenomenon is critical in any social science research project. In my thesis, I conducted a mixed methods case study that employs data triangulation. The discourse and content analysis of archived journals, online web survey of WPc community members and semi-structured email interviews of WPc members and management were augmented by information from my researcher's journal. This enabled me to address the research questions using multiple research techniques. The goal was to produce a study that is reflexive, collaborative, and ultimately of benefit to the virtual community I studied, as well as the extended and dispersed groups of women in both local and global communities who use new media technologies to engage in and address issues of common interest.

In this chapter I have laid out the methodological justification and strategy for this research, which is based primarily on virtual ethnography that employs a mixed methods case study. The next chapter will provide a brief review of Web 2.0 and the positioning of WorldPulse.com in that web. I also examine the affordabilities of Web 2.0, especially in the context of its interactive nature, which allows Internet users to challenge the gate-keeping and agenda-setting functions of traditional media. 
Chapter Four: The Internet and WorldPulse.com

Introduction

Over the last two decades, the Internet has become an essential part of public sphere activity. Many scholars have pointed out that the Internet has the potential to broaden public sphere involvement in comparison with older and less interactive forms of media, while other scholars caution against an utopian characterization of the Internet's potentials (Blumler and Gurevitch, 2001; Dean, 2003; Moe, 2008). Despite the intensive presence of commercial interest on the Internet, it is increasingly serving as a mechanism for civic engagement (Dahlgren, 2005; Wellman, Quan-Haase, Witte and Hampton, 2001; Volkmer, 2014). It has been argued (Papacharissi, 2002) that Internet technology allows for the formation of a global village, as proposed by McLuhan (1989). While these 'imagined' global communities are sometimes difficult to sustain, they periodically provide opportunities for otherwise marginalized voices to engage in debates about local and global issues.

Web 2.0

The Internet has become such a staple of everyday communication that, as far as the United Nations is concerned, "disconnecting people from the Internet is a violation of human rights" (Chipchase, 2011; Kravets, 2011). Chipchase observes that both individuals and communities are disadvantaged when they lack Internet access: 
In the United States, 92 percent of Americans rely on multiple sources for news, combining traditional print, TV, and radio and online sources, according to the Pew Internet and American Life Project, and among those who get news online, 75 percent receive their news via email or posts on social networking sites. 52 percent share news links with others via those sites. Statistics such as these suggest that those people who do not have access to the Internet are missing out; not only are we deprived of their voices, but also of their ability to learn about and interact with their communities and the world (Chipchase, 2011).

In terms of how the global interconnectedness of people is enabled by communication technologies, Quan-Haase and Martin (2013) suggest, by way of example, that Web 2.0 can foster "the emergence of a globally interlinked public sphere that takes advantage of the features of mobile media" (p. 3). Three particularly relevant characteristics of Web 2.0 audiences, as noted by Napoli are that they are: 1) autonomous - people having control over their own media consumption; 2) fragmented - no two audience members having the same experience; and 3) interactive - it allows and encourages people to respond to, and comment on, the media sources that they choose to view (2010, p. 6). In addition, QuanHaase and Martin write that:

One of the most important features of Web 2.0 is the ability of its users to participate in the media that surround them. This participation, whether it is adding photos to a person's Facebook page, or commenting on their Wall, [or in 
the case of the digital action campaigns of WPc, commenting on a person's journal entry] creates an interactive audience for those wishing to entertain, teach, or, for our purposes, encourage travel to new places (2013, p. 10).

\section{SEE THE PRINT VERSION FOR ACCESS TO THIS CONTENT}

A map illustrating the functionalities of Web 2.0 (O'Reilly, 2005)

This Web 2.0 meme map gives a visual representation of the collaborative opportunities available through the use of the Internet for communication. With regard to WorldPulse.com, the website functions as a web platform and site for publishing individual blogs: this allows members to simultaneously participate in social action through the digital action campaigns and also publish their writings in spaces that individuals co-create. The 
technology of WPc allows it to utilize the collective intelligence of its community members to produce content based on their personal experiences and interests. Beyond its use for participation in call to action campaigns, its members also use the WPc platform as a place for exchanging services that are useful for the communities and individuals to advance their work offline. These affordabilities of Web 2.0 on WPc help the latter to function as a site of public sphere activity.

WorldPulse.com on the Web

It is important to avoid essentializing the issues and relationships that are represented on WPc as a Web 2.0 platform. Web 2.0 is a technology with historical background. The technology on which Web 2.0 is built grew out of developments and advancements from Industrial Revolution machinery. Some of the elements and features of the 'old' technology are still evident in today's new technology, although they have been modernized to enable their presentation and resulting adoption as 'new' technology. Quan-Haase and Martin write that "in Web 2.0 environments, audiences have more control over what content to produce, how to present it, and where to distribute it" (p. 9). Web 2.0 is the architecture that facilitates many-to-many communication; it is a virtual space combining materiality and immateriality. It is a metaphorical space - like the public sphere - created by and through people's communication practices. Ideologies and discourses about new media emphasize how the Internet enables closeness, connection, interaction and the exchange of almost limitless amounts of information in real time. 
However, there are also concerns about the superficiality and ephemerality of these exchanges, and the extent to which they constitute what Lefort calls "invisible ideology" (1986). In an account clearly influenced by, and derived from, Debord's notion of The Society of the Spectacle (2006), Lefort writes that television, radio and other media technology produce:

A hallucination of nearness which abolishes a sense of distance, strangeness, imperceptibility, the signs of the outside, of adversity, of otherness ... no other phenomenon better illuminates the imaginary dimension of communication ... (the media) provides the assurance of the social bond, well removed from any test of its reality; it provides a background, an accompaniment ... just like the music which is rightly called by the same name ... and this background is the foundation ... for the intolerable fact of social division (p. 228).

There are many examples of the ways in which class and social positions still influence our relationships, whether online or offline; e-capital is entrenched in capitalism (DyerWitheford, 2002), and many civil society organizations often rely on sponsorship and partnership from corporate organizations. Such sponsorship and donor-donee relationships problematize the democratizing potential of Web 2.0, while also challenging the discourse of independence and autonomy advanced by civil society organizations such as WPc. Warner (2002) asserts that a public is independent of the state, and that this independence is an essential part of the formation and function of a public: "The way the public functions in the public sphere (as the people) is only possible because it is really a public of discourse. It 
is self-creating and self-organized, and herein lies its power, as well as its elusive strangeness" (p. 414). Publics are constituted through and by different forms and regimes of discourse and acts of interpellation. In identifying with a 'hailing' or act of address, a subject becomes, to some extent, a part of the public the address is targeting.

WPc is a public that also occupies a subaltern position (Warner prefers the term 'counterpublic'). The ways the community of women making up the membership carry out the practices of reflection in their journal writing and then the promotion of the women's writings - by WPc management - to mainstream media have the hallmark of subaltern, counter and or alternative publics. Such is WPc's awareness of its identity that one of its ways of encouraging its members to write in the journals is through the promise of the possibility of their writings being published in more visible mainstream media like CNN, Time.com and Huffington Post. Warner argues that "The cultural horizon against which it [the counter-public] marks itself off is not just a general or wider public but a dominant one" (p. 424). WPc often positions itself as the space for women to engage and address issues that are sometimes also being discussed on more visible, dominant media platforms. What marks WPc as subaltern is also predicated on the reason for the existence of the platform. In this context, the 'issues' criterion - which I will discuss in more detail in later chapters takes on an even more central position as a rallying point, since most WPc participants' performances on the platform cannot be differentiated from the practice of journal writing, and commenting on the issues discussed in those journals. This practice shapes and reshapes the WPc platform as a space or sphere for the members to constitute a particular public. 
In other contexts, it has been proposed that Internet access should be considered a social phenomenon because it enables an individual to engage in daily activities and interactions, some of which are now mostly facilitated by technological devices like mobile phones, tablets and computers (Liff and Shepherd, 2003). One needs to consider multiple factors when considering the digital divide, which remains an important factor and issue. The divide is not just about access to a technical apparatus, but also about the time and the skills needed to use the Internet. The authors observe that "both potential home access and work access ... were significantly less available to women than men" (p. 4). Liff and Shepherd argue that soft skills should be considered as equally important as accessibility: they argue that technical literacy is an important, and often overlooked, aspect of the digital divide.

We made the point that there is a popular conception that the Internet will increase civic engagement and participatory culture among people: however access to and ease of use of the Internet will not necessarily result in increased or more active participation in the public sphere. The Internet is only a technology, and technology can be used in a variety of ways; and it need not be used 'progressively'. By way of example, the current increase in the technologically facilitated connectedness of people and communities is accompanied, rather counter intuitively, by "a long-term decline since the 1960s in American civic involvement" (Wellman et al., 2001, p. 436-7). The most obvious way this decline manifests itself is in voter apathy, which is often measured at key moments such as during a federal election, when efforts are being made to 'get the vote out'. Despite these kinds of attempts to encourage voting among citizens, "the Centre for the Study of the American Electorate put 
2012 voter turnout at $57.5 \%$ of all eligible voters in America" (Liptak, 2012). Web 2.0 could change this trend by opening up channels for citizens to get involved in public sphere debates and political activities; and Wellman et al point out that "the Internet thus enhances the tendency for many ties to be nonlocal, connected by cars, planes, phones, and now computer networks" (2001, p. 438).

There are a number of online media forums set up by mainstream media organizations such as the BBC, CNN and the New York Times: these sites more or less supplement the preexisting established media formats of the media organizations, predominantly in the form of newspaper, radio, and magazines. Alternative forums such as WPc, which are gradually becoming mainstream in their membership and composition, are mainly set up by civil service organizations or other unofficial groups to facilitate discussions and deliberations. Often these forums claim to have no affiliation to any political party, and present themselves as nonpartisan. Questions could be asked about the extent to which both mainstream and online forums are critical, deliberative and constructive; in other words, how do they contribute to the democratic process, and inform policy debates? It might also be questioned whether such activity 'translates' into political action, and to what extent website management can address the challenge of ensuring inclusivity and equality for forum members. One of the functions of a forum as a subgenre of Internet websites is that it enables members to redefine what constitutes news. The 'Voices of Our Future' citizen journalism training, for instance, helps prepare its correspondents to fulfill this kind of function on both the WPc platform and mainstream media sites. Through this function, forum members can challenge, or at least provide an alternative to, conventional news 
gatekeeping and agenda-setting practices.

It is crucial to be able to assess the role of digital media in modern day social movements, and more generally as forums for public sphere activity. On the one hand, the Internet clearly constitutes an important series of sites and spaces from which to debate sociopolitical issues, and challenge the hegemonic control exercised over the conventional media by business organizations and what Bourdieu calls 'the field of power' (Bourdieu, 1996). On the other hand, the Internet and the field of digital media are themselves largely controlled and inflected by this field of power. Attempts to theorize and use digital media for the benefit of 'the people' continue to run into the realities of corporate and government management of all significant parts of the media; at the same time, these attempts are germane to the workings of any social democracy.

Arguably, the emergence of anti-capitalist movements such as 'The Anonymous', 'The Occupy Movement' and 'Wikileaks' could be read as a form of a democratic public sphere for contemporary society. The uprisings in the Middle East also provide evidence as to how digital media is utilized for popular political ends. However these movements, although horizontal and occasionally successful, are sometimes transient. The challenges faced by horizontal media organizations in gaining and retaining audiences online, in contrast with the control and breadth of audience attraction and retention exercised in and by the conventional vertical media, underline the difficulty of turning the sites of new and digital media into a functional and widespread alternative public sphere. 
The Comment is the News

With developments in digital technology, opportunities and channels for public communication and expression have widened, and people are taking advantage of these developments to articulate their views on social and political issues. Platforms that enable this expression are diverse, including Stuff.co.nz's comments feature; Radio New Zealand's the wireless.co.nz; CNN.com's comments feature; CNN's iReports; CBC.ca's comments feature; NigeriaVillageSquare.com; and similar sites in other parts of the world. Some of these platforms are available not just via their main websites, but also through mobile Apps on iPhone and Android-powered phones.

These facilities are available not just within vertical news organizations, but also with horizontal community organizations such as UN's WorldWeWant.org, WorldPulse.com, and other independently operated blogs. Twitter, for instance, can be considered as both a vertical and a horizontal organization. Arguably, it is an electronic forum that processes a massive amount of data daily; however, it also enables its users to respond to and discuss news stories. Through these channels, we get more information and insight into people's attitudes, and the issues that they consider to be important. However, moderation is still a significant issue and problem with these sites. Comments and contributions are often incoherent, incomplete, and unconsidered. While e-access does provide an opportunity for voices that have been excluded or marginalized, it requires a considerable amount of time and patience to read through the hundreds and sometimes thousands of comments on these 
sites. In some cases, significance is not to be found just in the headline or the story published on the platform, but in the comments written by the readers.

Recent examples of articles in which these practices manifest include the opinion piece titled 'The Curse of \#casualsexism' (Mau, 2015), and the news report titled 'Peter Golding's Pocket Pen Video Camera Keeps MPs Talking' (Van Dusen, 2015). Another way that this culture manifests is in reader-generated content that people upload online. Through social media sites such as Facebook, Twitter and YouTube, this content can be re-broadcast, go viral and reach global audiences. Through the use of digital technology and social media sites, people can redefine news culture and by extension influence or even constitute public sphere discussions. By way of example, a formal news article about healthcare in a well regarded media source - a newspaper or a multi-media organization such as the $\mathrm{BBC}-$ could be presumed to be reasonably well researched and to have utilized relevant sources (the Minister of Health, a non-profit health sector NGO, a relevant trained and qualified professional). After reading this story, when we review the comments accompanying the story - although not all stories get extensive comments - we may find some useful opinions, sources or issues that have been omitted; in other words, the commentary appended to the story can potentially extend, or point to limitations inherent in, the formal account provided. In research I conducted on the participatory culture of participants on the $\mathrm{CBC}$ News Politics forum in 2012, I identified readers' comments that were not directly related to the headline or contents of the article they were commenting on as "off topic" (Isekeije, 2012). These "off topic" comments have value in and of themselves, either in expanding on the issues addressed in the article or generating a stand-alone headline and article. 
The 'Voices of Our Future' Citizen Journalism Training Program

The process whereby readers and commentators discuss and attempt to critique, reorient, redefine or extend news stories is the focal point of the 'Voices of Our Future' citizen journalism training program facilitated by WorldPulse.com, which trains women to become citizen journalists. The website WorldPulse.com presents itself as a site for women to gather, discuss issues of common interest and concern; advocate for change; take action on the issues they identify as important; and incite, promulgate and effect policy changes on those issues. The website states that it is "bringing women a global voice. We are using digital media to connect women worldwide into a powerful force for change" (World Pulse, 2012). Members on the website express their opinions by writing in journals, blogs and comments, which are visible in open and accessible areas of the website. WPc considers itself a social media site for women who wish to form a collective voice, especially during its periodic digital action campaigns that focus on specific issues in diverse regions of the world. Its VOF correspondents then go beyond the digital action campaign journal entries and comments by producing numerous stories during their citizen journalism training program. Some of these stories are published as standalone in WPc online magazine, while others are published on platforms like the Global Press Journal and Huffington Post.

However, it is important to recognize that women are a heterogeneous group, not homogeneous in their dispositions, desires or needs. Being an active participant on an online forum does not necessarily translate to having agency; power differential is still 
present, although it is subtle and barely visible because of the horizontal positioning that the WPc management, who are also interacting on the platform, present to the participants. The general and generic discourse on WPc is mostly communal, employing and adopting a collegial and inclusive discourse that suggests the community of members jointly own all the resources of the organization. For instance, the founder often deflects any attempts by members to ascribe ownership to her. This deflection is often deployed in some of the posts and comments she makes on the website. How do some of the women on WPc develop the capacity to challenge power differentials and forms of symbolic violence? To answer this question, one needs to relate it back to historical contexts and political struggles. There is extensive information available on the WorldPulse.com website for both first time and repeat visitors, without requiring people to register to become a member of the community. The 'About Us' link provides an overview of what the organization is all about: "World Pulse is a nonprofit social media enterprise headquartered in Portland, OR, USA" (World Pulse, 2012). WorldPulse.com presents as a global community set up for women to use digital media in order to discuss issues and act on them to bring change to their communities.

One of the specific ways WPc fulfills its function is to encourage its members to use individually assigned sections of the website as a blogging platform and write extensively in their journals about issues of concern they observe in their communities. Dahlgren observes that there is an "increased deployment of journalistic modes for advocacy purposes" (2012, p. 101). WPc is an example of such platforms where journalistic modes are deployed for budding public intellectuals. Some would argue that this practice is more 
oriented towards advocacy journalism rather than citizen journalism. The journal writings are used for engaging in debates and deliberations about socio-economic and political issues. These journal writings are often converted and developed into full stories that are then promoted to and often published on mainstream media platforms like CNN and Huffington Post. Dahlgren asserts that "minimally, one can say that the web is redefining the premises and character of civic engagements for those who chose to engage" (p. 102). Those choosing to engage in this context would no doubt include budding public intellectuals engaging online on platforms like worldpulse.com. 
SEE THE PRINT VERSION FOR ACCESS TO THIS CONTENT

The new look homepage of WorldPulse.com; released February 2015. 


\begin{tabular}{|c|c|}
\hline SEE THE PRINT VERSION FOR & SEE THE PRINT VERSION FOR \\
ACCESS TO THIS CONTENT & ACCESS TO THIS CONTENT \\
\hline SEE THE PRINT VERSION FOR & SEE THE PRINT VERSION FOR \\
ACCESS TO THIS CONTENT & ACCESS TO THIS CONTENT \\
& The pre-February 2015 homepage; these \\
four versions show four images with diverse \\
information as the slider moves on the \\
homepage.
\end{tabular}




\section{SEE THE PRINT VERSION FOR ACCESS TO THIS CONTENT}

A map showing submissions from different regions of the world during the Ending Violence Against Women Digital Action Campaign in 2012.

\section{Conclusion}

It can be argued that the Web 2.0 facility helps provide more opportunities for collaboration among civic agents and civic engagement, although the complex issues of access and technical literacy problematizes these claims. WorldPulse.com's position in the increasingly complex web of digital information networks is central to its ability to fulfill its mandate of service as a global platform for women. Social and cultural capital, as well as a basic knowledge of political issues, is essential and forms part of the basics needed for 
effective civic activities that can produce desired results for the communities of people engaging on the platform. Based on my observation of the changing nature of media form and content over the last decade, I propose that 'the comment is the news'. By this, I mean that the opportunities citizens have - through the commenting features enabled on websites or blogs - to either change or subvert pre-planned agenda in public discourse inherently position participants as co-creators of news.

Moreover the issues commentators identify, when and if properly examined, usually indicate the missing voices that are oft marginalized in the reporting of news on mainstream platforms. Specifically with WPc, issues-driven activism linked to participants' comments expanding on issues initially placed on the agenda for discussion means that other participants have the opportunity to assess multiple and diverse dimensions of the issues and events that may have been overlooked. WPc's VOF citizen journalism training is a practical example of how female social activists are being equipped in the work of deploying journalistic modes for civic activities. During this training, the women get mentoring that develops their capacity to write not just one-directional journal reflections, but also to incorporate extensive, multiple views of diverse groups of people who are affected by the issues they identify. This training helps to ensure that some of the articles these women produce can be published on mainstream media platforms with a wider public reach.

With the rapid advancements in digital technology and the identification of the Internet as a staple by the United Nations, almost all major day-to-day activities, including educational 
pursuits, religious practices, banking, personal and household shopping, social relationships, as well as political involvement and participation, are now done online. The Internet offers opportunities for communication and collaborative work in many aspects of life, including areas such as communication, education, business, and political action. Platforms for civic engagement, such as WorldPulse.com, are also increasing their presence, and are helping to facilitate civic engagement.

The next chapter will provide a detailed report of the data obtained from archived journals of the digital action campaigns on WorldPulse.com, online web survey questionnaire and semi-structured email interviews, along with excerpts from the researcher's journal. The data sets provide an opportunity to conduct an extended critical assessment of the extent to which and how WorldPulse.com community members use the site. Data from the web survey and email interviews supplement the information that the WorldPulse.com community members produced during the digital action campaigns. 


\section{Chapter Five: Overview of the Research Data}

Introduction

Four sets of data are analyzed in this thesis. The first set comes from the digital action campaigns that were run on WorldPulse.com. The second set comes from an online web survey of the WPc community, which was announced on the website and was available from February 25 to May 25 2015. The third set comes from email interviews conducted with the World Pulse staff, digital action campaign participants, 'Voices of Our Future' citizen journalism training participants, and WPc general community members. The survey respondents were also asked to supply their email and Skype addresses if they wanted to be contacted for additional information to supplement the information they provided in the web survey. The fourth set is derived from a personal journal that I kept throughout the project.

This chapter provides a detailed report of the four phases of data collection, during which I used pre-existing journal entries and comments from the WorldPulse.com digital action campaigns, online web survey questionnaire, semi-structured email interviews and researcher's journal. All sets of data were collected over a three-year period from 2013 to 2015. Specifically the first set of data from the digital action campaigns was collected from 2013 to 2015; the second set of data from the survey questionnaire was collected from February to May 2015; the third set of data from the email interviews was collected from 
September to November 2015; and the fourth set of data from the researcher's journal was produced from 2013 to 2015 .

Content Analysis of the Digital Action Campaigns

The data reported in this section is from three digital action campaigns selected from WorldPulse.com. As of 9 May 2015, there were 49 items listed under the 'Take Action' link on the WPc website. I chose to assess the three items that had the highest number of journal entries. The three items, which I will continue to refer to collectively as digital action campaigns (or DACs) are: 'Ending Gender-Based Violence 2012' (EGBV) (this campaign was initially named 'Ending Violence Against Women' before the website was re-launched in February 2015); 'Girls Transform the World 2013' (GTTW); and 'Women Weave the Web 2014' (WWTW). Since the website was rebuilt and re-launched in February 2015, submissions made to the 'Voices of Our Future' (VOF) training program have also been listed under the 'Take Action' link. Since I initially set out to assess the 'Voices of Our Future' training program as a citizen journalism initiative, I am specifically excluding the VOF submissions from this phase of data selection. I will provide a close reading of the VOF campaign in phases 2 and 3 of data selection using the online web survey and email interviews. Following is a breakdown of the data selection for the Digital Action Campaigns 2012-2014. 


\begin{tabular}{|l|l|l|l|}
\hline \multicolumn{4}{|c|}{ Data selection for the Digital Action Campaigns 2012-2014 } \\
\hline \multicolumn{1}{|c|}{ Campaign titles } & $\begin{array}{c}\text { Total \# of } \\
\text { journal entries }\end{array}$ & $\begin{array}{c}\text { \# of selected } \\
\text { journal entries }\end{array}$ & $\begin{array}{l}\text { \# of comments } \\
\text { under selected } \\
\text { journal entries }\end{array}$ \\
\hline $\begin{array}{l}\text { Ending Gender-Based } \\
\text { Violence 2012 }\end{array}$ & 618 & 61 & 391 \\
\hline $\begin{array}{l}\text { Girls Transform The World } \\
2013\end{array}$ & 371 & 37 & 355 \\
\hline $\begin{array}{l}\text { Women Weave the Web } \\
2014\end{array}$ & 582 & 58 & 528 \\
\hline Total & 1571 & 156 & 1274 \\
\hline
\end{tabular}

Table 1: Data Selection for the Digital Action Campaigns

I selected $10 \%$ of the journal entries submitted for these three digital action campaigns to represent all the 49 items listed under the 'Take Action' link on WPc. Each post that a member initiated on the website and gave a title to is identified as a journal entry. Other members and the initiator of the journal entry can follow up this initial post with comments. The comments that are made below a person's journal entry do not appear in the journal of the people making the comments; instead, they are tagged along with the initial journal entry. I decided to count the number of entries selected based on initial journal entries, rather than both journal entries and comments. There are 10 journal entries on each page of the submissions as one clicks through the numbered links at the bottom of the pages. I selected every $10^{\text {th }}$ page and took all the 10 journal entries on the page, except for the last 
page, from which I selected the numbers that are less than 10 (i.e. 8 journal entries for EGBV, 1 journal entry for GTTW and 2 journal entries for WWTW).

Some of the journal entries were written in French, Spanish and Arabic. I used the Google Translate app on the website to convert these entries to English prior to selecting and including them in the data. Volunteer community members of WPc translated some of the non-English entries, and these members are credited for their work. For such journal entries, a notice was posted to indicate that a WPc community member translated the entry. An example of this practice is the journal entry under WWTW that reads: "English translation by community member McKinley" (this notice appears under the journal entry by muhorakeye on 13 June 2014).

Some of these journal entries consist of pre-existing literary works such as news stories, book excerpts, poems, pictures, and short stories: not all of them were written by the WPc community members. I have coded and analyzed these entries, and examined the issues raised in them as part of the submissions and responses to the campaigns under which they appear. In some cases, these secondary and pre-existing literary works appear to help the WPc members articulate what they otherwise could not write themselves. Some of the community members have given credit to the original authors, while others have not. An example is the poem on Kate Fletcher by Kathy English: the WPc community member Hekima Place posted the poem in her journal for the GTTW campaign; she writes "Kate Fletcher inspires me. Who inspires you?" (GTTW 3). 
Ending Gender-Based Violence 2012 (EGBV)

For the 'Ending Gender-Based Violence' digital action campaign of 2012, there were 618 individual journal entries. I selected a total of 61 journal entries to code and analyze. There were 391 posts commenting on these 61 journal entries. These comments were included in the data selection for coding and analysis. I analyzed all the comments under each of the 61 journal entries. This digital action campaign is still referenced as 'Ending Violence Against Women 2012' in the introduction to the journal entries, but listed as 'Ending Gender-Based Violence' in the list of 'Take Action' campaigns. I will continue to refer to this campaign as 'Ending Gender-Based Violence 2012' (or EGBV). The summary in the introduction to this campaign indicates that a total of 153 WPc community members contributed to the 618 journal entries.

SEE THE PRINT VERSION FOR ACCESS TO

THIS CONTENT 
The lead image for the 'Ending Gender-Based Violence

2012’ digital action campaign.

Most of the journal entries in this campaign (the first 10 entries) had less than 10 comments each. It appears to be early days in community and forum interaction on the WPc platform: possibly this was because it was before the WP management started pairing journal entries and commentators. This pairing is referenced as part of the researcher's journal discussion of my experience as a part of the WPc volunteer community. Briefly, one of the tasks I completed as a WPc volunteer was to be matched with journal entries that I then had to comment and provide feedback and ratings on, through a Google Doc form. This was backend voluntary work that was coordinated by the WP management team. In other contexts, I am aware that some online platforms employ and contract people to make comments on postings on their platform in order to boost the level of customer and community engagement on their websites. Mostly profit-inclined platforms will pay for this service. For WPc to pay for this service would clearly raise ethical issues; however, there are also questions to ask when volunteers are recruited and paired to make comments on specific journal entries in the digital action campaigns. I will address this issue in the data analysis section.

I noticed that there are some differences between the translation given by the community volunteers and the translation supplied by the Google Translate software. For the posts that have translation from community members, I decided to code these translations instead of 
the Google Translate version. For those with no translation from community members, I decided to code the Google Translate version.

SEE THE PRINT VERSION FOR ACCESS TO THIS CONTENT

A screen shot of a journal entry: it indicates that the journal is publicly available; gives the writer's profile name and the number of comments accompanying the particular journal entry; has a flag showing which country the writer declared as his or her location; provides a photograph of the writer (some community members do not have their real pictures on their profiles while others use avatars); and gives options for accessing the entry in the languages offered by the Google Translate app. 


\section{SEE THE PRINT VERSION FOR ACCESS TO THIS CONTENT}

This is an indication and acknowledgment that a community member has translated the journal entry into English. These translators are volunteer community members on WorldPulse.com.

Girls Transform The World 2013 (GTTW)

For the 'Girls Transform the World' digital action campaign of 2013, there were 371 individual journal entries. The comments under each journal entry are additional to this number. I selected a total of 37 journal entries to code and analyze. There were 355 posts commenting on these 37 journal entries. All these comments were included in the data selection for coding and analysis. I analyzed all the comments under each of the 37 journal entries. The summary for the GTTW campaign supplied by WPc indicates that there were "more than 350 submissions from more than 60 countries" (GTTW 1). 


\section{SEE THE PRINT VERSION FOR ACCESS TO THIS CONTENT}

The lead image for the 'Girls Transform The World 2013' digital action campaign.

Comments under most of the journal entries in the 'Girls Transform the World 2013'

campaign were slightly longer in comparison to the 'Ending Gender-Based Violence 2012'

campaign. This observation is based specifically on the data selected for this project.

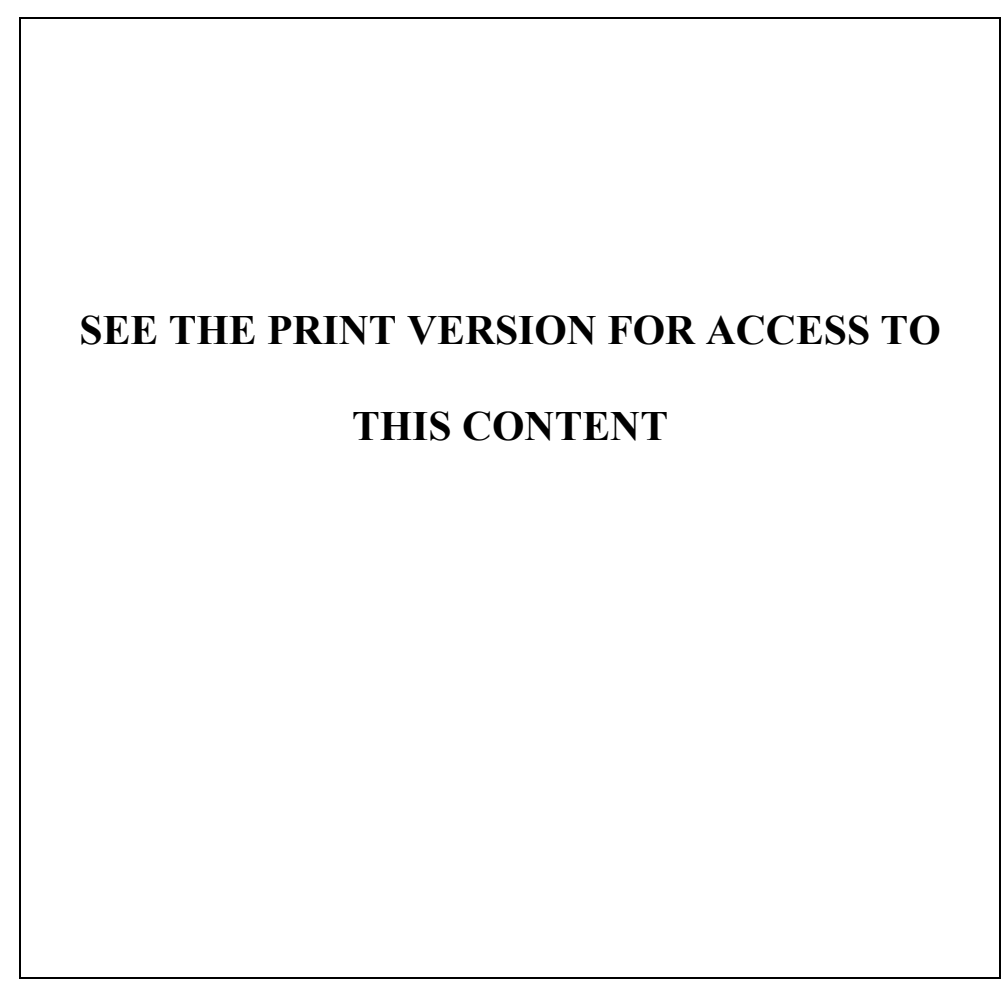


Nicole Musimwa's profile picture in the 'Girls

Transform the World 2013' digital action campaign.

Women Weave The Web 2014 (WWTW)

The 'Women Weave the Web' digital action campaign of 2014 had 582 individual journal entries. The comments under each journal entry were additional to this number. I selected a total of 58 journal entries to code and analyze. There are 525 posts commenting on these 58 journal entries. All these comments were included in the data selection for coding and analysis.

SEE THE PRINT VERSION FOR ACCESS TO THIS CONTENT

The lead image for the 'Women Weave the Web 2014' digital action campaign. 
The campaign summary indicated that there were a total of "590 submissions from nearly 70 countries around the globe" (WWTW 1). This campaign had more comments under most of the journal entries, although some journal entries still recorded less than 10 comments each. I participated in this campaign as a volunteer commentator, and was assigned specific journals to comment on. One of the tasks assigned to volunteer commentators was to rate the comments based on the criteria the WP management supplied to the volunteers. Some of the data selected in this campaign have multiple journal entries by the same author; all these entries were included in the selected data.

\section{SEE THE PRINT VERSION FOR ACCESS TO THIS CONTENT}

Map of safe spaces identified by community members during the 'Women Weave the Web 
2014' digital action campaign.

Online Web Survey of the WorldPulse.com Community

The online web survey was made available to the WPc community on 25 February 2015, when I also posted a notice inviting community members to participate in the survey. The notice was posted in the 'Leadership' and 'Economic Empowerment' groups on February 25 2015. I also posted the notice in my journal as a WPc community member. Between February 25 and March 8, I sent private messages using the mailing system available to members of the community to WPc members, encouraging them to participate in the survey. As a WPc community member, I am allowed to send out a maximum of five messages to 50 recipients per day. I specifically included all the 'Voices of Our Future' training participants, their Vision Mentors, Editorial Mentors, Editorial Midwives, and Mentors in the private messages. I wanted to include the input of the entire 'Voices of Our Future' group representing all the years that the program has been running.

‘Voices Of Our Future' Citizen Journalism Training Program

The 'Voices of Our Future' (VOF) citizen journalism training program was first offered in 2009 and has been running since then. For the 2012 and 2013 versions of the program, there are interactive maps available on the website. One can click on each of the pins on the map to read the profile of the correspondent from the country represented on the map. The 
first two screen shots below show the maps for 2012 and 2013 correspondents. The next three screen shots show the profiles of the correspondents from Afghanistan, Mexico and Nigeria. The 'Voices of Our Future' is:

An online training program investing in women's ability to use new technology and media to share their stories and innovative solutions with the world. World Pulse and program partners the Press Institute, the OpEd Project, and the Empowerment Institute provide training in web 2.0, citizen journalism and mentoring to empower a new generation of grassroots women leaders to raise their voices, promote their visions for change and become leaders for their communities (World Pulse, 2012).

The information available on the WPc platform shows that 124 correspondents participated in the four sessions of the training program in 2009, 2011, 2012 and 2013. 


\section{SEE THE PRINT VERSION FOR ACCESS TO THIS CONTENT}

A screen shot of the interactive map showing the location of the correspondents in the 2012 'Voices of Our Future' citizen journalism training program.

SEE THE PRINT VERSION FOR ACCESS TO THIS CONTENT

A screen shot of the interactive map showing the location of the correspondents in the 2013 'Voices of Our Future' citizen journalism training program. 


\section{SEE THE PRINT VERSION FOR ACCESS TO THIS CONTENT}

A screen shot of Rabia.Salihi's profile. Rabia is from Afghanistan. She is one of the 2013 'Voices of Our Future' correspondents. 


\section{SEE THE PRINT VERSION FOR ACCESS TO THIS CONTENT}

A screen shot of Klaudia Mexico's profile. Klaudia is from Mexico. She is one of the 2013 'Voices of Our Future' correspondents.

\section{SEE THE PRINT VERSION FOR ACCESS TO THIS CONTENT}

A screen shot of Vweta's profile. Vweta is from Nigeria. She is one of the 2013 'Voices of Our Future' correspondents. 
On 28 February 2015 WPc community member Nwamini commented on the survey invitation that I posted as a journal entry 'resource exchange': the comment was written in French. Using the Google Translate app, I converted the comment into English to access the information. Nwamini wanted to respond to the survey in French. I thought about making the survey available in French and Spanish, the other two commonly used languages on the website. The Qualtrics software I used for the online web survey has a multiple language facility; however, I decided to restrict the survey to English because of the issues around converting the responses from other languages back to English. The challenge of the language barrier is an issue that I will return to in later chapters.

I issued survey invitations to all the 31 members in my friends list on WPc. These members have diverse identifiers in their profiles, including 'community welcomer', 'community listener', 'vocal contributor,' and 'voices of our future [with year indicator; for example 2009] correspondents'. The WPc private message tool allows only five messages per day. I spread the task of issuing the survey invitations over two months; the last set of invitations was issued on 8 May 2015. Over the course of these two months, I sent friend requests to the VOF correspondents, their mentors and editorial midwives, and their vision mentors. Some of the vision mentors are volunteers from World Pulse's partner organization, the 'Empowerment Institute'. Some of the VOF editorial midwives are correspondents who had gone through the VOF training in previous years. A small number of the mentors and editorial midwives were not registered as members on the WPc website, and hence they were not accessible through WPc; accordingly I could not invite them to participate in the 
online web survey. I limited the survey invitations to WPc community members who could be reached through their WPc profiles.

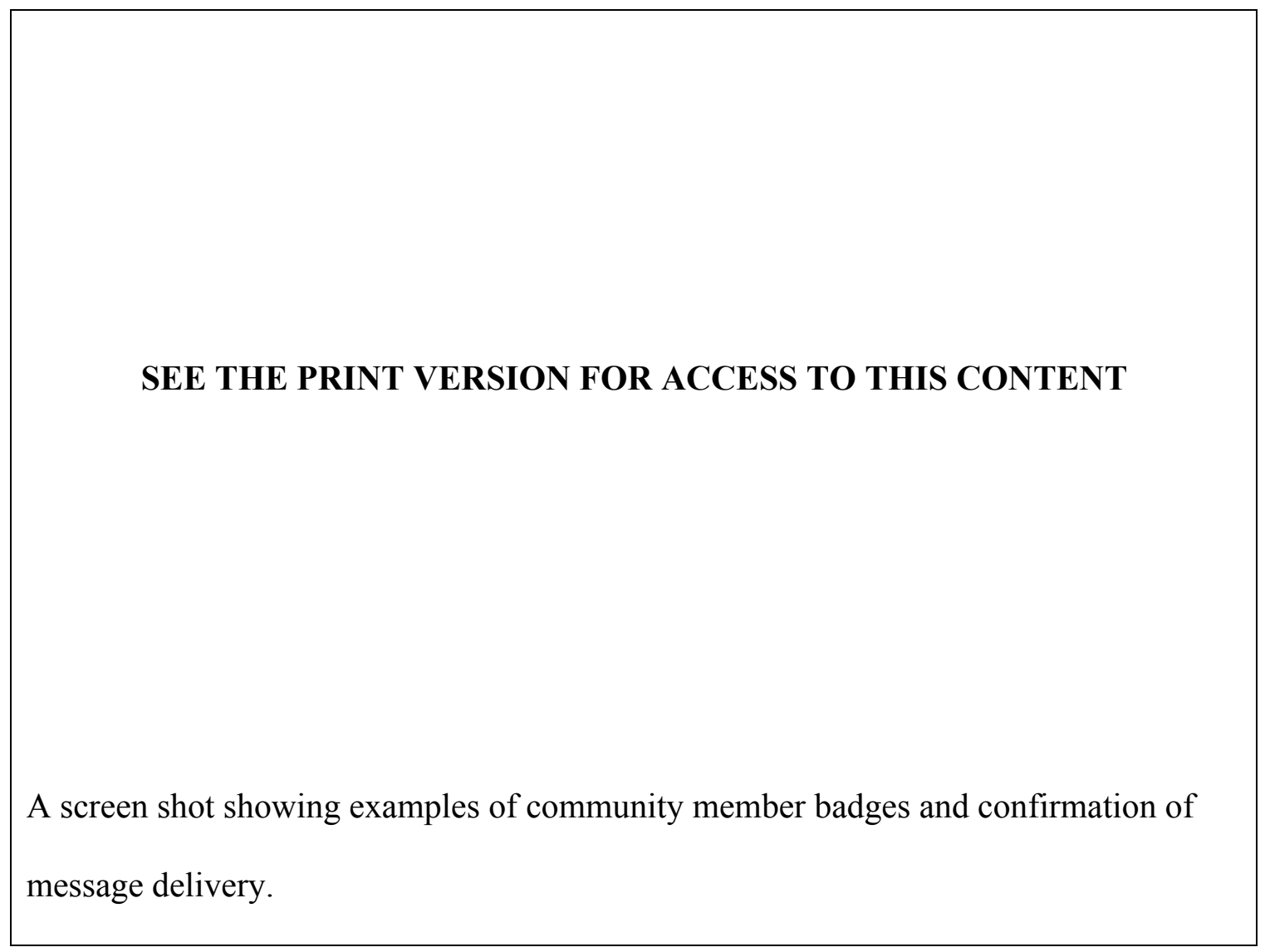




\section{SEE THE PRINT VERSION FOR ACCESS TO THIS CONTENT}

A screen shot showing examples of community member badges and confirmation of message delivery.

I issued more survey invitations through the private message tool on WPc, and found that there was a corresponding increase in the number of people taking the online web survey. Some of the restrictions placed on the WPc private messaging tool included the limit of 128 characters in the "To" field for each message. Even though one can send invitations to 10 people per message, depending on the number of characters in their profile names, it is not always possible to get 10 people into each message. There were a few occasions when I could only include eight or nine people in a single message. I included non-VOF community members in the survey invitations, as the 'autofill' automatically showed their names while I was trying to find specific people in the VOF groups. 
WPc had 22,800 members from over 190 countries in its community as of late 2015 (WP Staff 1). This information was useful for determining the percentage of the WPc community members who started, as compared to those who completed, the survey; the Qualtrics report showed that 62 respondents started the survey and 46 respondents completed it. A manual review of the survey responses indicated that one additional respondent left only a few questions unanswered: these responses were included in the final selection of the survey data. A total of fifteen responses that had been submitted during the pilot phase of the survey were deleted, because they either did not answer any or all of the questions. Forty-seven responses were included in the final selection of responses. The survey data analysis is based on these 47 responses, which represents $0.21 \%$ of WorldPulse.com's registered members.

The review of the survey results indicates that 23 of the survey respondents have applied for the VOF training program. There are 124 correspondents altogether across the 2009 to 2013 training sessions. Out of the 23 VOF applicants, 22 indicated that they had undertaken the 'Voices of Our Future' citizen journalism training. This number 22 represents $18 \%$ of the total number of VOF correspondents across the years that the training was offered on WPc. 


\section{SEE THE PRINT VERSION FOR ACCESS TO THIS CONTENT}

A screen shot of the message that I sent to invite participation in the online web survey.

Computer-Assisted Software: NVivo and SPSS

Computer-assisted data analysis software was utilized in this dissertation. Banner and Albarran's (2009) research offers some useful ideas regarding computer-assisted qualitative data analysis. The authors discuss the use of such software, including NVivo, in their study and argue that "the primary function of analysis software is the effective archiving, management and storage of the vast quantities of raw data generated through the qualitative research process" (p. 25). They identified two types of computer-assisted qualitative data analysis software (CAQDAS): the first type is for coding and analysis while the second 
type is for building theory. Both types of CAQDAS found expression in my research project. In applying Habermas' categories of assessing communicative action, and DiMaggio and Hargittai's categories of assessing broad forms of inequality, I used NVivo and SPSS for coding and analysis. In developing the embedded e-activists' civic engagement typology, I used NVivo and SPSS for coding and analyzing the data.

I imported the DAC data by copying each journal entry and the accompanying comments manually from the WPc website, pasting them into a Word document, and then importing the Word file for each of the three campaigns into NVivo for coding. I created a total of 16 Word files for the EGBV, GTTW and WWTW campaigns, and used these files for coding and analyzing the DAC data. NVivo was also used for coding and analyzing the data from the online web survey and email interviews. SPSS was used specifically for testing and interpreting the online survey data. Both NVivo and SPSS were used for developing the themes for the civic engagement typology. 


\section{SEE THE PRINT VERSION FOR ACCESS TO THIS CONTENT}

Picture accompanying Rebecca R's journal entry for the 'Girls Transform the World 2013' digital action campaign.

Online Web Survey Report

The online web survey report includes responses from a total of 47 participants. The demographics of these participants are as follows: gender - five male, 41 female and one undeclared; age -17 respondents in the 18-30 year age bracket, 11 respondents in the 31-40 age bracket, while 19 respondents were 41 and above; marital status - 24 respondents were single, 17 were married, four were in common law relationships while two were divorced; location - 20 respondents were based in Africa, seven in Asia, one in Australia, four in Europe, 13 in North America, one in Oceania and one undeclared; level of education - 19 respondents had Bachelor's degree, 24 postgraduate degrees, one had secondary level education, two had trades qualifications and one was undeclared; occupation/profession - 
41 respondents were in paid employment, one was retired, one unemployed, three were volunteers, with one undeclared; income -35 respondents declared their income indicating a mean income of US $\$ 26,804.57$. Below is a breakdown of the demographic information supplied by the survey respondents. See Appendix 2 for the detailed report generated from the online web survey data.

\section{Question 1: What is your gender?}

\begin{tabular}{|ll|r|r|r|r|}
\hline & & Frequency & Percent & $\begin{array}{c}\text { Valid } \\
\text { Percent }\end{array}$ & $\begin{array}{c}\text { Cumulative } \\
\text { Percent }\end{array}$ \\
\hline Valid & Male & 5 & 10.6 & 10.9 & 10.9 \\
& Female & 41 & 87.2 & 89.1 & 100.0 \\
& Total & 46 & 97.9 & 100.0 & \\
Missing & System & 1 & 2.1 & & \\
Total & & 47 & 100.0 & & \\
\hline
\end{tabular}

Table 2: Survey participants' gender

Table 2 shows that $10.6 \%$ of the sample was male, $87.2 \%$ was female while $2.1 \%$ did not state their gender. Having five males among the 47 respondents of the survey is significant considering that WPc appears to be primarily targeted at women. The valid percentage in this table only accounts for those who responded to the question; it does not account for the one individual who did not declare gender. 


\begin{tabular}{|c|c|c|c|c|c|}
\hline & & Frequency & Percent & $\begin{array}{c}\text { Valid } \\
\text { Percent }\end{array}$ & $\begin{array}{c}\text { Cumulative } \\
\text { Percent }\end{array}$ \\
\hline \multirow[t]{4}{*}{ Valid } & $18-30$ & 17 & 36.2 & 36.2 & 36.2 \\
\hline & $31-40$ & 11 & 23.4 & 23.4 & 59.6 \\
\hline & $41+$ & 19 & 40.4 & 40.4 & 100.0 \\
\hline & Total & 47 & 100.0 & 100.0 & \\
\hline
\end{tabular}

Table 3: Survey participants' age

Table 3 shows that $36.2 \%$ of the sample was aged between 18 and 30, 23.4\% between 31 and 40, while $40.4 \%$ was 41 years of age or older. This indicates that the majority of participants on WPc are in the age bracket of $18-30$ and $41+$, the latter slightly more than the former age group. 
Question 3: What is your marital status

\begin{tabular}{|c|c|c|c|c|c|}
\hline & & Frequency & Percent & $\begin{array}{l}\text { Valid } \\
\text { Percent }\end{array}$ & $\begin{array}{c}\text { Cumulative } \\
\text { Percent }\end{array}$ \\
\hline \multirow[t]{5}{*}{ Valid } & Single & 24 & 51.1 & 51.1 & 51.1 \\
\hline & Married & 17 & 36.2 & 36.2 & 87.2 \\
\hline & $\begin{array}{l}\text { Common } \\
\text { law }\end{array}$ & 4 & 8.5 & 8.5 & 95.7 \\
\hline & Divorced & 2 & 4.3 & 4.3 & 100.0 \\
\hline & Total & 47 & 100.0 & 100.0 & \\
\hline
\end{tabular}

Table 4: Survey participants' marital status

Table 4 shows that $51.1 \%$ of the sample was single, $36.2 \%$ married, $8.5 \%$ was in a common law relationship, while $4.3 \%$ was divorced. More than half of the participants are single. 
Question 4: Which region of the world do you live in?

\begin{tabular}{|c|c|c|c|c|c|c|}
\hline & & Frequency & Percent & $\begin{array}{c}\text { Valid } \\
\text { Percent }\end{array}$ & $\begin{array}{c}\text { Cumulative } \\
\text { Percent }\end{array}$ & Countries \\
\hline \multirow[t]{8}{*}{ Valid } & Africa & 20 & 42.6 & 42.6 & 42.6 & $\begin{array}{l}\text { Cameroon (1) } \\
\text { DR Congo (3) } \\
\text { Kenya (1) } \\
\text { Liberia (1) } \\
\text { Nigeria (10) } \\
\text { South Africa (1) } \\
\text { Tunisia (1) } \\
\text { Uganda (2) }\end{array}$ \\
\hline & Asia & 7 & 14.9 & 14.9 & 57.4 & $\begin{array}{l}\text { Kashmir (1) } \\
\text { Lebanon (1) } \\
\text { Myanmar (1) } \\
\text { Philippines (3) } \\
\text { Saudi Arabia (1) }\end{array}$ \\
\hline & Australia & 1 & 2.1 & 2.1 & 59.6 & Australia (1) \\
\hline & Europe & 4 & 8.5 & 8.5 & 68.1 & $\begin{array}{l}\text { Austria (1) } \\
\text { Netherlands (1) } \\
\text { Romania (1) } \\
\text { United Kingdom } \\
(1)\end{array}$ \\
\hline & $\begin{array}{l}\text { North } \\
\text { America }\end{array}$ & 13 & 27.7 & 27.7 & 95.7 & \begin{tabular}{|l} 
Canada (1) \\
United States of \\
America (12) \\
\end{tabular} \\
\hline & Oceania & 1 & 2.1 & 2.1 & 97.9 & New Zealand (1) \\
\hline & Undeclared & 1 & 2.1 & 2.1 & 100.0 & Undeclared (1) \\
\hline & Total & 47 & 100.0 & 100.0 & & \\
\hline
\end{tabular}

Table 5: Survey participants' location

Table 5 shows that $42.6 \%$ of the participants were from Africa, $14.9 \%$ Asia, 2.1\% Australia, 8.5\% Europe, 27.7\% North America, and 2.1\% Oceania; and 2.1\% did not state their 
location. Almost half of the respondents (20 out of 47) were from Africa with 10 out of that number located in Nigeria. More than a quarter of the respondents (13 out of 47) were based in North America, with 12 out of that number located in the United States of America. The third largest group (7 out of the 47) was from Asia, with the Philippines accounting for three out of those seven respondents.

Question 5: What is your highest level of education?

\begin{tabular}{|c|c|c|c|c|c|}
\hline & & Frequency & Percent & $\begin{array}{c}\text { Valid } \\
\text { Percent }\end{array}$ & $\begin{array}{c}\text { Cumulative } \\
\text { Percent }\end{array}$ \\
\hline \multirow[t]{6}{*}{ Valid } & Bachelors & 19 & 40.4 & 40.4 & 40.4 \\
\hline & Postgrad & 24 & 51.1 & 51.1 & 91.5 \\
\hline & Secondary & 1 & 2.1 & 2.1 & 93.6 \\
\hline & Trades & 2 & 4.3 & 4.3 & 97.9 \\
\hline & Undeclared & 1 & 2.1 & 2.1 & 100.0 \\
\hline & Total & 47 & 100.0 & 100.0 & \\
\hline
\end{tabular}

Table 6: Survey participants' level of education

Table 6 shows that $91.5 \%$ of the participants had a postsecondary level of education, 4.3\% had trades certification, $2.1 \%$ had a secondary level of education, while $2.1 \%$ was undeclared. A significant percentage (51.5\% or 24 out of the 47 respondents) had a postgraduate level of education. 
Question 6: What is your occupation/profession?

\begin{tabular}{|l|r|r|r|r|}
\hline & Frequency & Percent & $\begin{array}{c}\text { Valid } \\
\text { Percent }\end{array}$ & $\begin{array}{c}\text { Cumulative } \\
\text { Percent }\end{array}$ \\
\hline Valid Paid & 41 & 87.2 & 87.2 & 87.2 \\
Retired & 1 & 2.1 & 2.1 & 89.4 \\
Undeclared & 1 & 2.1 & 2.1 & 91.5 \\
Unemployed & 1 & 2.1 & 2.1 & 93.6 \\
Volunteer & 3 & 6.4 & 6.4 & 100.0 \\
Total & 47 & 100.0 & 100.0 & \\
\hline
\end{tabular}

Table 7: Survey participants' occupation/profession

Table 7 shows that $87.2 \%$ of the participants were in paid employment, while $6.4 \%$ were in volunteer roles. The fact that $87.2 \%$ (accounting for 41 out of the 47 respondents) were in paid employment is significant, especially considering the amount of time they contribute to the campaigns on WPc.

Question 7: How much do you earn per year?

\begin{tabular}{|l|r|r|r|r|r|}
\hline & \multicolumn{1}{|c|}{$\mathrm{N}$} & Minimum & Maximum & Mean & Std. Deviation \\
\hline $\begin{array}{l}\text { How much do you } \\
\text { earn per year? }\end{array}$ & 35 & 0 & 185000 & 26804.57 & 33984.923 \\
Valid N (listwise) & 35 & & & & \\
\hline
\end{tabular}

Table 8a: Survey participants' income

Table 8 shows that for those individuals in paid employment, the mean income was USD $\$ 26,804.57(\mathrm{~s}=33,984.92)$. This is quite a large standard deviation that is possibly attributable to the fact that people would not give their exact income, but instead rounded it out. 


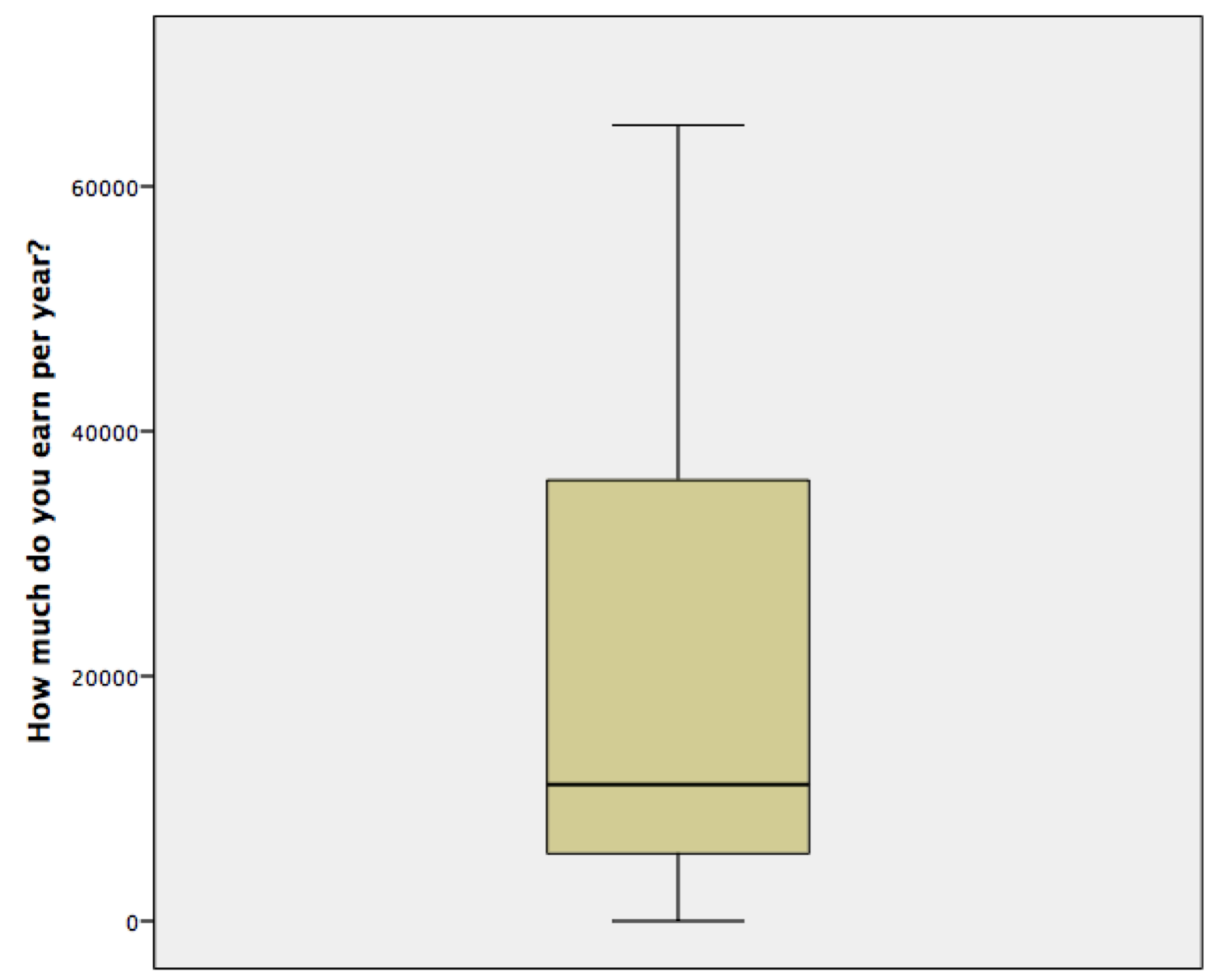

Report

How much do you earn per year?

\begin{tabular}{|c|r|c|l|}
\hline Mean & N & $\begin{array}{c}\text { Std. } \\
\text { Deviation }\end{array}$ & Median \\
\hline 22151.76 & 34 & 20231.466 & 11150.00 \\
\hline
\end{tabular}

Table 8b: Survey participants' income, after excluding those earning more than US\$100,000 per year.

After excluding those earning more than US $\$ 100,000$ the bottom $50 \%$ earn less than US\$20,000/annum. The median is US\$11,150 among the whole group. One participant indicated an annual income of US\$185,000. 
Email Interviews with WorldPulse.com Community Members and World Pulse Staff

These interviews were conducted by email. There were four groups of people involved. The first and second groups included respondents to the online web survey. The first were survey respondents who had indicated they participated in the digital action campaigns and would like to participate in follow up interviews. The second were survey respondents who had indicated they participated in the 'Voices of Our Future' training and would like to participate in follow up interviews. In some cases, there were overlaps between these two groups of interview participants. All the survey respondents in groups one and two provided their email addresses for me to contact them to conduct the interviews. For these two groups of participants, I sent an introductory email that included a reminder that they had indicated interest in the follow up interviews when they took the online web survey.

The third group included WPc community members who either wrote journal entries or commented on journal entries in the three digital action campaigns that I assessed in the first phase of data selection. These WPc community members had included their email addresses in the signatures on their journal entries or comments. For this third group of interview participants, I sent an introductory email informing them that I obtained their contact details through the digital action campaigns on WorldPulse.com. The fourth group was World Pulse staff members representing the organization. I sent a reminder email to the staff members who I had communicated with about the research project and who had indicated to me that I could conduct interviews with them via email. 
I invited a total of 41 people to participate in the interviews: 22 people were from groups one and two, 15 people from group three and four people from group four. A total of 19 people from groups one to three accepted my invitation and agreed to participate in the interviews, while two people from group four participated. At the close of the interviews there were 10 interview participants in total who responded to the questions. One participant withdrew due to ill health, while some others did not state their reasons for not completing the interviews. The responses of all the 10 participants who completed the interviews have been included in the final data selection and analysis. My goal at the beginning of the interviews was to have about $8-10$ interview participants out of the total of 47 online web survey participants.

The interviews were conducted over 14 weeks from August to November 2015. For the first three groups, the interview questions were divided into four sections covering technology and autonomy, skill and social support, digital action campaigns and 'Voices of Our Future' training. For the fourth group, the interview questions were divided into four sections covering staff profile, WorldPulse.com profile, digital action campaigns and 'Voices of Our Future' training. Over the 14-week period, I sent questions covering each section to the interview participants, and gave them a one-week response deadline with an additional note that they could send their response in sooner, if they were so inclined. Most of the participants responded within the given deadline; I sent reminder emails to the others asking them to send in their responses. In some cases, I sent in additional follow up questions based on the information received in the responses to the initial set of questions. 
Overall, the 10 interview participants identified as one male and nine females, and they were located in Colombia (1), the Democratic Republic of Congo (1), India (1), Lebanon (1), Nigeria (2), and the United States of America (4). Names of all interview participants have been changed to conceal their identities. The locations of the interview participants were not being matched to the assigned pseudonyms or ages to further protect them because of the number of the participants. The age range of the eight participants was $24-67$. Two of the interview participants were staff members of WorldPulse.com, and they were identified in the project as WP staff 1 and WP staff 2; while the other eight participants are WorldPulse.com online community members. The eight community members were identified as Nkem, Emeka, Chika, Uloma, Amara, Ifeoma, Funanya and Ogechi.

Technology and Autonomy

Responses to interview questions about technology and autonomy indicated that each of the eight participants have multiple devices in the forms of mobile phones, tablets, laptops and desktops, except for one participant who has only one device - a laptop. All participants indicated that these devices belong to them. While most participants have autonomy of use of the devices, one indicated that she shared her desktop - which is one out of the four devices she can use to access the Internet. Six participants reported a positive experience that was articulated mostly around the autonomy of use and ownership of multiple devices, which gives them room for flexibility with Internet access. One participant noted that she has become more reliant on her mobile phone in 2015 and this reliance has impacted 
negatively on her participation on WPc and other websites. Another participant noted that her netbook - one of two devices she owns - "sometimes it gets hang and typing a lot of stuff at a stretch is little bothersome" (Ifeoma). All eight WPc community members have access to WPc while at home. In addition to home access, two of the interview participants access WPc at work, three at cyber cafés and one relies on her mobile for access when she is travelling. Six participants indicated that the site of access when using WPc either did not affect or had a positive effect on their interactions on the website, while one participant's site of access (cyber café) had a negative effect. One participant did not respond to this question.

\section{SEE THE PRINT VERSION FOR ACCESS TO THIS CONTENT}

Gladys Shumbambiri's picture to illustrate her journal entry titled 'Digital Tools and Women in Zimbabwe' for the 'Women Weave the Web 2014' digital action campaign.

Skill and Social Support 
With regard to issues of skill and social support, all eight interview participants reported diverse levels of satisfaction they obtained from their ability to access the Internet generally and WorldPulse.com specifically. Those who had participated in the digital action campaigns and 'Voices of Our Future' training indicated they were satisfied overall with their level of skill and the social support they receive while interacting on WPc. Chika responded as follows:

In my local society it is very difficult to do a change by yourself as a person. When interacting with other women-worldwide-who share similar ideas, I feel that my viewpoints are sound and can be applied to improve the status of women. In the VOF program you feel that someone is appreciating your voice, and if it's not sharpened yet, someone is encouraging you to raise up your voice. This idea that from more than 500 women worldwide I have been selected is by itself amazing. Someone believes in me, or at least believes I have the potential (NR).

All the participants engaged in various voluntary activities on WPc, including completing tasks involved in occupying formal positions such as Community Listener, Community Champion (Economic Empowerment group), Community Advisory Board member, Community Champion (Gender-Based Violence group), and World Pulse representative at public speaking engagements. All participants indicated they were comfortable using the primary language of communication, which is English, while one participant indicated 
some difficulty with one of the other languages of communication: "Most conversations are in English and I am okay with that. Some in French, I can read and understand, but wouldn't reply in French" (Emeka). Seven of the participants indicated they have not experienced any difficulty accessing WPc; one indicated she had experienced difficulty and got a swift response to support her when she needed the support. Two of the participants indicated they know who to ask if they experience a difficulty, and specifically mentioned their knowledge of an existing "welcome booklet" (Emeka) and "online toolkit" (Amara) for troubleshooting on the platform. Three participants articulate how the assistance and support available on WPc encourage them to continue using the platform:

Amara: The assistance made me to accept WP as my own community. A refuge in time of sorrow. A home where I'm always welcome and a family where I can share my burden (NR).

Ifeoma: It really does help me to personally stay connected with the WP, when there are people who always care for you and wants to provide every kind of help to make easy our usage or access. If I would be interested to participate in a program and there would be no assistance or help that would automatically discourage me and I might leave things half-way (NR).

Ogechi: Without World Pulse's support we would continue only beating the air with all our intentions. No one would know about us 
and no one would have an opportunity to join in our fight; which solidarity has greatly strengthened not only our resolve, but our impact... Because of the availability of the busy staff and World Pulse, we know and experience our connection and our value as part of the force that is changing the world women live in, right where they live (NR).

\section{SEE THE PRINT VERSION FOR ACCESS TO THIS CONTENT}

Carolepng reports during the 'Women Weave the Web 2014' digital action campaign that she is Empowered Online to Bring Impact Offline.

\section{Digital Action Campaigns}

Two of the interview participants did not participate in the digital action campaigns on WPc. Six of those who had participated in the campaigns describe their experiences using various 
affirmative words including "fruitful, original, positive, encouraging" (Chika); "educational, enlightening, informative" (Uloma); "great” (Amara); “exciting journey, incredible opportunity, connecting, relevant" (Ifeoma); "interesting" (Funanya); and "exhilarating" (Ogechi). All six WPc community members have participated in at least one DAC, while one has participated in four campaigns, and another in six campaigns. One participant is aware of the presence of a moderator who coordinates each DAC. Some of the interview participants also indicated they have received information about the outcome of the DACs, either through general member email notifications, postings on the platform, or via specific mailing directed to an individual member.

The interview participants identified advantages and disadvantages with regard to online activism, including the Digital Action Campaigns of WPc. In terms of advantages, Chika referred to "exposure, sharing different perspectives, hearing voices from different backgrounds, and spreading the message online" (NR). For Uloma it was "knowing that others have similar challenges and being able to share solutions to those challenges, making connections with others who can help point you to resources or create solutions, creating a common language around global issues that affect people locally" (NR). Amara suggested that "these voices reach where our legs could not reach. Policy change happens in some countries. The campaigns reach more audiences and policy makers faster" (NR). Ifeoma wrote that "the most important advantage was the idea of global connection, where in the virtual medium, we can connect with the global leaders and see how they are making a difference" (NR). For Ogechi "the advantages are that you really find out what you yourself think on the specific issue, and get the perspectives of your colleagues as well as others 
around the world. As well we feel we are participating in a global outcry on the specific Campaign topic to bring more attention to the issue. Another benefit is the visibility our participation gives us with our sisters around the world, and that sometimes comes back to our authorities as the [cyber café] and/or specific members are mentioned" (NR). WP staff 1 provided a more extensive and detailed summary:

There are a lot of advantages to running online action campaigns. First of all, they are accessible to any person, anywhere in the world as long as they have internet access. Secondly, action campaigns give people a variety of ways to be involved with a topic that is important to them and also connects them to a community of others who have this same passion and interest. Someone can engage with something as simple and accessible as signing a petition (which can have incredible impact), adding a "safe space" location to a global map, or they can invest in writing a thoughtful and provocative piece. Third, our action campaigns give us the opportunity to partner with others for a bigger vision and cause, increasing all of our effectiveness. For example, [community member's name and group] from the Democratic Republic of the Congo's petition was the tipping point in appointing Senator Feingold as Special Envoy to the DRC (NR). 


\section{SEE THE PRINT VERSION FOR ACCESS TO THIS CONTENT}

The image Anakarenart used to illustrate her journal entry titled 'The World Needs Latin America and Mexico Women in Tech' during the 'Women Weave the Web 2014' digital action campaign.

With regard to disadvantages, Chika suggested that "at many times this may not be the whole picture in the specific region or country, at some points we may be encouraged online-virtually, but when it comes to the ground it is much harder to implement projects by yourself" (NR). Uloma made the point that "it is easy to simply offer platitudes and praise without actually taking action, can feel a little detached from people's real problems by only reading about them" (NR). For Ifeoma "the disadvantage is that still in our part of the world, technology is not that accessible and strong which makes it still a difficult step to spread the word on various issues and empower more and more women or connect with the deserving lot!" (NR). Funanya wrote that "being digital I think they can not touch my real 
life" (NR). For Ogechi "a disadvantage can be personal security depending on the sensitive nature of the topic and the openness with which the participant approaches the topic" (NR).

'Voices of our Future' Citizen Journalism Training Program

Two of the interview participants did not participate in the 'Voices of Our Future' training on WPc. Out of the six who had participated, five were correspondents while one was an editorial midwife/mentor. All six who had participated in the training referred to their experiences affirmatively, describing it as: "of a special kind" (Chika); "very wellorganized, easy to follow instructions, very easy to understand what to do" (Uloma); a "great experience ... triggered me to become a better empowered women" (Amara); "really worthy, widen my network of women, create a sort of global resource pool" (Ifeoma); "incredible experience" (Funanya); "exhilarating, it was really great" (Ogechi). One of the six respondents participated in the VOF in 2009, another two participated in 2011, two participated in 2012 while one participated in 2013. All five VOF correspondents indicated they had written articles for publication since they took the citizen journalism training. Four stated they have had their articles published on multiple platforms, while one was not aware of any of her articles being published but recollected that "I have been quoted, interviewed and written about many, many times" (Ogechi). Four participants reported that they had not been paid for any of the articles they wrote for publication on WorldPulse.com; while one reported that she was paid for one article that appeared in the website's monthly magazine. 
Interview participants identified advantages and disadvantages of the citizen journalism training. For Chika the advantages included "sharing experiences with people worldwide and cooperating to solve major problems" (NR). Uloma wrote about "hearing voices we often don't hear from perspectives we are often unfamiliar with" (NR). Ifeoma pointed out that "one can write as many articles as one wants to and on any theme without any restriction" (NR). For Ogechi "the advantages are that you get to speak about what is important to you; what you care about. Also you are typically not viewed as a threat to anyone" (NR). WP staff 1 identified three advantages: "(1) Accessible to any woman, anywhere in the world, as long as she has internet access. (2) We can have 30 women from 30 different countries participate in a training together, learning from one another, sharing, and helping support one another's transformation. (3) It is a very cost effective way to deliver training to a global audience of powerful leaders" (NR).

With regard to disadvantages, Chika suggested that "Maybe the biggest one is the inability of implementing some solutions on a personal level. Some-many-of the problems need collective effort" (NR). For Uloma "writers have very limited points of view, which can sometimes mean they are disregarded by the media on the whole ... is that writers do not get paid for their work as opposed to other journalists, so their work is sometimes seen as 'less than"' (NR). Ifeoma wrote that "the disadvantage is that people do not take you that seriously and do not trust you that much as in professional journey of journalism" (NR). Ogechi pointed out that some people "don't have any financial support for equipment or internet costs, let alone living costs" (NR). WP staff 1 again identified three points: "(1) It takes a lot more effort to create a sense of deep trust and connection with a group online 
versus in person. (2) Internet reliability is always a challenge to negotiate. (3) Personality and cultural misunderstandings can happen more easily when a training is primarily writing based than when people are in person together and can read body language, etc" (NR).

When discussing their media literacy prior to participating in the 'Voices of Our Future' training, three of the correspondents indicated they had some background in journalism, one said that she had a "good" media literacy (Funanya), one said that she had "very poor" media literacy (Ogechi); while the editorial midwife indicated she had "exceptionally high" media literacy (Uloma).

Researcher's Journal

I have been a registered community member on WorldPulse.com since 2009, and have taken an academic research interest in the platform since I first started postgraduate studies. I however did not get a chance to conduct research on the WPc community until 2013 when I started this research project. From 2013 I was not just a community member on the platform, but also became a participant-observer conducting virtual ethnographic research. Between 2013 and 2014, I volunteered as a Community Listener in the 'Voices of Our Future' citizen journalism training program and the 'Women Weave the Web 2014' digital action campaign. I used a researcher's journal primarily for documenting my research. I was fortunate to have a serendipitous encounter with the global \#bringbackourgirls campaign while conducting my research. I actively participated in this campaign, which included organizing and leading rallies in New Zealand to draw attention to the plight of 
the young women for which the campaign was initiated. I plan to draw on some of the information in my researcher's journal to develop another project that could be used for testing the civic engagement typology I developed in this research.

Specific to the WPc digital action campaigns, an excerpt from my researcher's journal on 26 June 2015 reads:

I would like to honour the women who wrote the Digital Action Campaign journal entries and whose personal account of issues helped to illuminate the challenges they face in their various communities, and in particular the journal entries which record violence against women. In all three digital action campaigns, even though they each focused on different agenda items, the issue of violence against women came up in most of the journal entries, especially the ones submitted by women from the Southern Hemisphere. Arguably there is no mechanism in place to verify the accuracy of some of these narratives, but I nonetheless felt the weight of the experiences the women talked about and wondered many times about what kinds of support they got to assist them in dealing with these issues.

I should mention here that there are some journal entries that I omitted quoting in the analysis chapter because the raw information was just too graphic. Some of these journal entries referred, in a detailed manner, to sexual assaults and various forms of the violation of children. 


\section{Conclusion}

For the content analysis phase of this project, there were 1571 journal entries produced between 2012 and 2014 during the three digital action campaigns: 'Ending Gender-Based Violence 2012', 'Girls Transform the World 2013', and 'Women Weave the Web 2014'. A total of 156 of these journal entries and 1274 comments accompanying the journal entries were selected for coding and analysis in this project. The online web survey allowed access to obtain contextual data, most especially from WPc members who had participated in the organization's citizen journalism training initiative named 'Voices of our Future'.

Computer-assisted software, SPSS was used specifically to analyze the quantitative data produced through the online web survey. A second computer-assisted software, NVivo, was used for analyzing the qualitative data from the survey as well as the content analysis and email interviews. NVivo was particularly useful for developing the inductive civic engagement typology for embedded e-activists.

The email interviews with WPc members revealed that most of the participants had technological access and enjoy a relative amount of autonomy of use of their electronic devices. Interview respondents' levels of skill and experiences of social support varied across all the eight interview participants. The diversity of technological competence and experiences were further distinguished by participants' involvement in the DACs and VOF. The two WPc staff who participated in the interviews provided an official account of the organization and additional contextual information about the digital action campaigns and the VOF training program that I would not have been able to access without the 
organization's input. Keeping a researcher's journal throughout this project was useful for documenting my experiences across the multiple stages of the process of planning the research project as well as completing the work. Most especially I found the journal to be an effective tool during my participation in the \#bringbackourgirls campaign, which employed both online and offline tactics - a reflection of the work that embedded e-activists do in local and global communities.

The pre-existing archived journals available on the WorldPulse.com website from the digital action campaigns, conducted over three years, provided an extensive amount of data that allowed for a broad assessment of the practices on the website. Some of the journal writings also captured the offline work that most of the writers did beyond their online activities. The online web survey questionnaire was designed to collect data that was not readily available in the journal writings, most especially demographic information about the journal writers that they would otherwise not have included in their journals. The unstructured email interview questions further complement the data produced from the journals and survey by using a one-to-one system of communication for an exchange between the interviewer and interviewee. The data indicates the diversity of the WPc community, and suggests that even though the site is primarily targeted at women, men participate as well. There is also an element of self-critique that emerged in the data that indicates the WPc community members were aware of the imbalance between site owners and users. This self-critique mostly occur among the WPc community members. 
The next three chapters will undertake a critical analysis of the data introduced in this chapter, using a combination of discourse and content analysis and ethnographic descriptions of the culture and practices of the website, contextualized by and tested against the analytical frameworks of Habermas, DiMaggio and Hargittai, and the civic engagement typology that I developed while conducting this research. Habermas' public sphere theory has been extended by Dahlberg so as to offer a methodological model for assessing public sphere sites and practices. The five broad forms of inequality identified by DiMaggio and Hargittai provide categories of analysis that are accompanied by individual hypotheses. The civic engagement typology that I use to analyze the data in Chapter eight developed organically as I worked through the sets of data reported in this chapter. The typology is proposed as a model for assessing civic engagement practices among an advanced crop of citizen journalists who I identify as embedded e-activists. 
Chapter Six: Public Sphere Activity and Engagement on WorldPulse.com

Introduction

In the next three chapters, I will conduct a critical analysis of the combined data collected from the journal entries and comments in the digital action campaigns, the online web survey, semi-structured email interviews and researcher's journal. By extension, the chapters will emphasize the ways in which the culture of WPc produces particular forms of 'between us'. This culture of the specific kind of 'between us' will be described, analyzed, evaluated and compared to the various Habermasian prescriptions about what constitutes a public sphere site. I will provide an account of what the site is trying to do, which groups are involved, and what kinds of 'rules of engagement' are encouraged and naturalized. This will lead to an argument regarding the (re)evaluation of the extent to which Habermas' prescriptions are useful, and the extent to which we can identify a single and relatively homogeneous public sphere culture or regime, in the Habermasian sense, or whether this site and its activities indicates that the public sphere needs to be thought of as a plurality of spaces, each one with its own rules of engagement and imperatives.

In this chapter, I use the data collected through the digital action campaigns, online web survey and email interviews to assess Habermas' public sphere prescriptions, in the context of the changes occurring in discursive activities and events as a result of technological advancements. In Chapter Seven, I critically analyze the data collected through the DACs, online web survey and email interviews using the five broad forms of digital inequality 
identified by DiMaggio and Hargittai. In Chapter Eight, I introduce a civic engagement typology, which will assess the data primarily collected from the digital action campaigns. Where relevant, I also include data from the online web survey and email interviews. Information from all four phases of data collection - the archived journal entries and comments from the DACs, online web survey, semi-structured email interviews and researcher's journal - are critically analyzed throughout the three chapters.

The DACs covered in these three chapters ran between 2012 and 2014. These campaigns were: 'Ending Gender-Based Violence 2012' (EGBV); 'Girls Transform the World 2013' (GTTW); and 'Women Weave the Web 2014' (WWTW). The EGBV campaign was part of the annual commemoration of the campaign for eliminating violence against women, an international day the United Nations has set aside for drawing attention to this issue. The GTTW was linked to the 'Day of the Girl-Child' commemoration, another United Nations' international day. The WWTW campaign alerted multiple stakeholders to the opportunities and challenges the Internet holds for women. The online web survey and email interview questions asked respondents to reflect on some of the work they did when participating in the DACs and the 'Voices of Our Future' citizen journalism training program. Respondents were also asked to reflect on their practices generally on WorldPulse.com, including the reasons why they participate on the website, what value they placed on their participation, where they accessed the website from when they participated in the campaigns and training, and what policy changes they have observed in their local communities that are linked to the campaigns on WorldPulse.com. 
Public Sphere Prescriptions

In previous chapters, it was argued that Habermas' criteria of the public sphere are conceptualized as ideals that civil society should be working towards. It was also established that the revisions Habermas made to public sphere theory in communicative action theory recognizes that discursive activities and events are assessed within the sociocultural and political contexts in which they occur, rather than being evaluated as abstractions. Within this context, there is an acknowledgement that interlocutors' 'interests' are diverse and, given that the public sphere clearly extends across globally networked spaces, subject to the specificities and influences of time and place Habermas accepts and recognizes that the reality of public sphere activity, particularly as it is played out within the context of new media technology, does not match up with any set of idealized abstractions. However Ragnedda and Muschert (2013) suggest that Habermas' criteria are still a useful starting point for assessing communication in the public sphere; and while Volkmer (2014) argues that Habermas' 'ideal speech' situation is not a sufficient basis for assessing public sphere activity across multiplied sites, spaces and sets of interest, she suggests that Habermas' criteria can still be used, in combination with other analytical models, to good effect.

It is within this context that I used the deliberations, engagement and interactions of forum members on WPc to assess the usefulness of the prescribed ideals of the public sphere, while recognizing that the theory's limitations would require an assessment of the dimension of digital divide and digital inequality. Volkmer (2014), in her assessment of 
conflicting arguments about the viability of a global public sphere, alerts us to "a shift from 'rationality' to a softer sphere of 'experience"' (p. 178). In this context, Volkmer alerts us to the role of emotionality in public discourse. This observation shows why it is important to examine the intersection of deliberative discourse and digital inequality. If experience is useful in communicative discourse and practices, and part of digital inequality show a gap in skills needed for engaging online, combining Habermas' public sphere theory and DiMaggio and Hargittai's digital inequality model constitutes a practical way of analyzing and evaluating the kinds of communicative practices on WPc. The following section of this chapter provides a critical account of the public sphere concepts of autonomy, exchange and critique, reflexivity, ideal role taking, sincerity, and discursive inclusion and equality, while assessing their applicability to the discursive practices occurring on worldpulse.com.

Individual Agency and Independence of Site of Discourse

Habermas' normative expectation of the public sphere requires that it should be independent from state and economic power. The expectation relates to the autonomy of participants and public sphere sites. For Habermas, "Discourse must be based on the concerns of citizens as a public rather than driven by the media of money and administrative power that facilitate the operations of the market and state" (Dahlberg, 2001, p. 2). This autonomy functions on two levels: one concerns the level of autonomy of individual participants, while the other refers to the autonomy of the platform that is being used for coordinating public sphere deliberations. The WPc platform is an independent, non-profit and non-governmental organization that is funded through donations and grants 
from individuals, corporate organizations and contestable government and international donor grants; the site publishes its list of donors on its platform. Despite the independence claimed by WPc, there are relationships and connections to donors that the platform needs to maintain. In Habermas' terms the website is not entirely autonomous.

As an organization that relies heavily on donations, there is the possibility of implicit (and perhaps even explicit) influences directly or indirectly from the sources of the funding. It is not unreasonable to suggest that WPc may occasionally have to tailor its agenda to fit the demands of certain donor organizations. The platform's independence from the government - in this case the government of the United States of America, since WorldPulse.com is registered in that country - is not in question since it has a registered charity status, and is expected to operate independent of government influence. However, expectations do not always meet reality: there have been cases of non-governmental organizations becoming politicized for the benefit of groups of people who want to use them to 'ideologize' members of the organizations. A case in point is 'Planned Parenthood', which has often had its funding become a source of contestation as a result of partisan politics in the United States. 


\section{SEE THE PRINT VERSION FOR ACCESS TO THIS CONTENT}

Image accompanying Olutosin's journal entry titled 'Weaving My Words and World on the Web' for the 'Women Weave the Web 2014' digital action campaign.

In examining the autonomy of forum members, articulation of individual autonomy varied from one interview participant to the other. The interview data indicated that each participant was representing his or her own interest and were not on the website as a representative of any business or government institution. However, a uniformity of autonomy expressed by eight of the 10 interview participants focused primarily on ownership of multiple devices for accessing WPc and other Internet websites. The autonomy that is expressed around ownership of technological devices and digital access will be taken up in chapter seven.

By extension, Habermas' acknowledgement in communicative action theory that autonomy is not an absolute requirement (although still preferred over some form of entanglement 
with the political state and economic influence) opens up a space for WPc to fulfill its public sphere roles without its reliance on sponsorship and government funding being considered an impediment. In the same vein, Habermas' warning that 'systems' media have colonized 'lifeworld' media problematizes WPc's position as a site with qualitative value and influence that can stand in the space of civil society and challenge the hegemonic power and money of political state. If some of the platform's funding comes indirectly from powerful sources (who also have roles in government, for example the website's tie to The Clinton Foundation) then the language and issues WPc employs will be modified to ensure that these sources of funding are not compromised.

In the contemporary context of the breadth of digital media usage, and more specifically of the ways in which independent media sites that are set up for civic engagement rely on multiple sources for financial support, the notion of autonomy as an indispensible criterion for the legitimacy of public sphere space and activity is highly problematical. Many such sites need to be linked into broader economic frameworks and networks, and this means that they may not be completely independent or autonomous. In this context, socio-cultural institutions, including the commercial media, can function as public sphere sites. Foucault draws our attention to the fact that individuals and institutions are never outside the operations of power, but are tied into and influenced by networks of power relations. The commercial link that a website such as WPc has for the purpose of sustaining its operations, does not delegitimize the work being done in that space. Almost all the media sites that are accessible through Internet technology rely on some kind of financial sponsorship model 
that can potentially compromise their ability to claim absolute separation from political and economic influences and forces.

Rationality and Emotionality

The second of Habermas' criteria focuses on the issue of rational-critical critique, which "involves engaging in reciprocal critique of normative positions that are provided with reasons and thus are criticizable - are open to critique rather than dogmatically asserted" (Dahlberg, 2001, p. 2). A key marker of genuine civic engagement, for Habermas, is the possibility of a critique of moral-practical validity claims. Tully (2013) argues that, "when subjects engage in the difficult yet commonplace practice of turning and reflecting on an aspect of the relationships of power, knowledge, and subjectification that govern their conduct, they bring that aspect into the immediate public sphere they create" (p. 185). This practice was not particularly evident during the WPc digital action campaigns. Although community members often posted reflections in their journals, while also making references or linking their stance on issues to extended discussions outside of the WPc space, the extent to which these activities could be characterized as critique, in the Habermasian sense, was both limited and rare. Rather, there was an extensive form of engagement that indicated the participants' vulnerability as emotional, reflexive expressions overtook any form of rational approach to the issues being discussed. Participants did provide feedback in the form of literary works, news stories and excerpts from biographies. In some cases, however, community members further developed their journal entries by linking their literary works to key policies and debates on the issues 
being discussed. In the GTTW campaign, Sutanuka Banerjee responded to another member, Cali gal Michelle's comment regarding her post about stereotypes in India's school curricula: she contributed a post identifying the issue of gender stereotypes in young children's books in India, and argued that this stereotyping is a systemic issue that is reinforced by what the curriculum expects teachers to deliver in classrooms (GTTW 5). Sutanuka then extended this argument to take into consideration how such early exposure to stereotypical gender roles reinforced the suppression of women's voices in the wider public sphere.

What is foregrounded here is the performance of a kind of socio-cultural literacy. This does not constitute, nor is it commensurate with, the notions of sincerity and authenticity, two other public sphere criteria that are somewhat related and will be discussed later on in this chapter. Rather, this performance elaborates upon and develops the issues at hand, and shows how Cali gal Michelle both identifies with the proposition that this is a serious community issue, and demonstrates how the issue can be taken elsewhere, and considered and understood from wider perspectives. To further connect her previous post to these issues as they occur in the wider public sphere, Sutanuka then contributed the following post in the comments section, listing in detail the internationally endorsed position on this issue:

The invisibilization of women in the public space is a serious issue.

I followed the report on eliminating gender stereotypes in the EU based on Beijing Declaration and Platform for Action adopted by 
the Fourth World Conference on Women on 15 September 1995. It enlisted that: whereas children are confronted with gender stereotypes at a very young age through role models promoted by television series and programmes, discussions, games, video games and advertisements, study materials and educational programmes, attitudes in schools, the family and society, which influence their perception of how men and women should behave and which have implications for the rest of their lives and their future aspirations, education and training.

P. whereas sexist stereotypes are conveyed both by teachers (willingly or not) and by the educational support material teachers are given; Q. whereas access to formal primary, secondary and third-level education and the content of the curriculum as taught to girls and boys is a major influencing factor on gender differences and, correspondingly, on choices and access to rights (GTTW 5).

Sutanuka further expands the discussion, making links to issues of inequality, stereotyping and gender wage gap disparity in the workforce:

R. whereas the notion of equality can be instilled in children at a very young age and an upbringing where equality is acknowledged can teach them to combat gender stereotypes; 
S. whereas the stereotypes which still exist with regard to the educational and professional options available to women help to preserve inequalities; whereas education and training continue to transmit gender stereotypes, as women and men often follow traditional education and training paths, and this has serious repercussions on the labour market, limiting career diversification and often placing women in occupations that are less valued and remunerated;

T. whereas in the education process boys and girls are still not encouraged to take an equal interest in all subjects, in particular as regards scientific and technical subjects;

U. whereas, although many European countries do include a gender dimension in their career guidance, this is normally aimed at girls to encourage them to choose careers in technology or science, and there are no initiatives to encourage boys to consider careers in education, health or the humanities;

Labour market

$\mathrm{V}$. whereas the impact of gender stereotypes on education and training has strong implications for the labour market, where women still face both horizontal and vertical segregation W. whereas gender stereotypes on the labour market still limit women's access to certain sectors, such as engineering, firefighting, manufacturing, construction, carpentry, mechanics, the 
technical and scientific sectors and new technologies, but also limit men's access to childcare sectors (midwives, nursery nurses, etc.) (GTTW 5).

A discussion between three WPc members featured a more explicit example of exchange that functions as a form of critique (GTTW 33). Sophie posted the initial journal entry to which Diane responded; and then Sophie followed up with additional comment, which Jill then expanded on. Implicit in Jill's comment is the point that WPc community members sometimes are diplomatic and purposely employ inclusive and sensitive language, as they have to straddle the thin line separating the supportive discourse of WPc and the practical expectations guiding critical engagement in a public space:

Comment by Diane Ezeji: I really enjoyed your article. You gave many examples that show how one turn in the path of life can make such important differences. I don't think it is a matter of "luck" though. If that was so, it would be random and we wouldn't see that certain characteristics that a person holds either promotes them or holds them back. For example, if you are a minority person living in a country that holds prejudice against this group of people, do you think you are given the same opportunities as the main, powerful group in that place? If you grow up in a culture that doesn't see the value of educating women, would being born a woman give you an unfair advantage? I also liked how you wrote 
that what someone at first thought was bad, turned out to be something good. Someone forced to go to school when they'd rather be with the other boys herding cattle. I think life can bring us difficulties that end up teaching us valuable things (GTTW 33).

Comment by Sophie: My struggle or definition of 'luck' is that like in the example you have given if born in a place where you are marginalised then you are 'not lucky' haaa meaning you might suffer discrimination that are not really within your control hence if another one is born in place where other factors held constant they have the opportunities then they are lucky to be born there..hmmm or is it fate?? :) (GTTW 33).

Comment by Jill Dulitsky: Thank you for your beautiful essay. It was so thought provoking! My head is swirling with information. I think you hit on a topic that we are sometimes afraid to address and I agree with you. Most times being born into priviledge is just luck. I was recently in Haiti for a week and I came home with the same feelings that you have. Boy, am I lucky! My family told me from the day I was born that I would go to university. It was never a question. That is lucky. I have also read the book the Outliers and loved it. Ever so often, though, there is someone that comes into my life that has overcome 
the "luck equation". I went to university with a girl who was raised by a single mom and her abusive boyfriend in the slums of St. Louis, MO. She moved out on her own at 15 years old because she witnessed her mom being beaten almost to her death. She worked, paid rent and went to highschool. She graduated in the top of her class and got a full scholarship to unversity. She broke that chain. But, one of the reasons that she was able to break it, is that the United States, as a whole, promotes education for women. I can't imagine living in a place that culturally didn't believe in it. Thank you for sharing. Keep the thoughts coming...together we can shift the world's perspective to make more girls "lucky". I look forward to reading your blog! (GTTW 33).

Linda M. Ando's comment on Mambo's journal entry in the EGBV campaign continues and develops this exchange:

Yes, it is time to end the violence against the women and children in the Eastern DRC. The healing needed for the women \& children to recover and rebuild their lives does require a comprehensive approach, beginning with the most essential needs. Mental, physical and economic healing are needed. Are you a part of a community group or organization to apply for micro-credit loans? I agree to empower the women with financial means to 
survive and create community based programs which directly services the women \& children is essential (EGBV 33).

The expectation that public sphere interlocutors should engage in 'serious' discussions undergirded by logical and rational reasoning is both a strongly delimiting prescription, and highly impractical in terms of the levels of commitment that people bring, on an everyday basis, to socio-cultural issues and agendas. This logic also extends to the format of agendas, where the expectation is that participants should have identified issues for discussion prior to commencing those discussions. In many socio-cultural exchanges and discussions, it is not possible to delimit, control or predict where an argument or exchange will go; moreover, it seems more democratic to have the schedule of issues decided within a discussion, rather than being identified and pre-determined by a few people. The idea that only certain issues should be considered of public interest fails to take into account that some issues, such as children's rights and domestic violence, are of importance to marginalized groups but get minimal attention because they are often considered to be private rather than public issues.

The requirement of rational-critical engagement continues to be a major cornerstone in Habermas' work and body of theory. His firm commitment to this prescription is reflected in the extensive outline he provided for it in his theory of communicative action (1981) and discourse ethics (1990). This requirement of rationality stands in opposition to emotionality, which is sometimes considered to be less valuable than rational logical reasoning in argumentative discourse. Rationality assumes that participants have a fixed identity and speak from a unilateral position that is firmly supported by logical reasoning and can be 
supported through the attachment of proof of validity claims. However, a fixed and unified identity is not tenable when we consider that individuals have diverse socio-cultural influences that affect their appearance in public. By extension these influences show up in the diverse expressions and manifestations of emotionality, which reflects their identity as always in flux and subject to the contexts and sites of discourse.

The opportunity to challenge the status quo in public sphere sites is very limited, and complicated by the economic circumstances of many of those engaging in public sphere activity. In the case of WPc, since most participants would want to have the opportunity to extend their forum membership to further benefit their individual goals or for further improving their local communities, it would be difficult for them to challenge the positions and power differentials on the platform. By extension, their ability to challenge the status quo on the issues of deliberation is subject to the official position of the WPc platform, which is linked to the organization's socio-economic and political commitments. This holds an advantage for the platform since it is set up primarily to draw attention to issues that do not often find expression in mainstream, and often male dominated, public sphere sites.

The rational-critical debate is less evident in theoretical discussions of public sphere activity, and the scholarship tends to focus, instead, on the concepts of reflection and reflexivity (Dahlgren, 2012; Poster, 2001; Volkmer, 2014;). Markell (1997) also notes that consensus is no longer required for the discussion of issues of common interest in a public sphere. Habermas acknowledges that the contestation and reconsideration of issues is an ongoing process, and that consensus need not be achieved prior to the commencement of 
deliberations. He no longer considers the lack of consensus as a weakness, but rather positions it as a necessary feature for public sphere deliberation.

\section{SEE THE PRINT VERSION FOR ACCESS TO THIS CONTENT}

Image accompanying Sister Zeph's journal entry titled 'I Open Girls' Minds' during the 'Women Weave the Web 2014' digital action campaign.

WP staff usually introduces digital action campaign topics, and promote the topics to the community members by asking them to make submissions indicating their personal experiences with the topic on the agenda. These topics are then left open for community members to make their contributions. There is no particular format regarding moderation: this means that there is no 'guidance' of the discussion in a way that might encourage exchange and critique, as opposed to journal entries and comments that often appear to stand alone, rather than build on previous points or aspects of the discussion. This informal and unstructured system is beneficial for ensuring individuals have a certain level of 
autonomy in their contributions. Perhaps such an unstructured system also impacts negatively on the extent to which arguments can be rational-critical or contributions can be 'developed' and responded to during the campaigns.

\section{Reflexive Engagement}

Another public sphere prescription requires that, "Participants must critically examine their cultural values, assumptions, and interests, as well as the larger social context" (Dahlberg, 2001, p. 2). Habermas remains committed to this expectation, although as we have seen in many interactions in virtual space the ways in which reflexivity is manifested, when it is identifiably present, can be quite diverse, depending on the socio-cultural, economic, and political contexts. Foucault argues that people's world views are produced through, and informed and delimited by, power relations; and reflexive thinking and processes cannot be divorced from the naturalized discourses of 'truth' being circulated in a socio-cultural space. These 'discourses of truth' define and delimit the kinds of reflexive processes available to subjects.

The proliferation of intensely personal communication on the Internet further problematizes the ideal of reflexivity; in their attempts to be reflexive, most participants end up sharing personal information that are not necessarily related to the issues being discussed and may not have any influence on the outcome of the discussion. There is very little evidence of reflexivity in the existing data gathered from the DACs on WPc. What would have otherwise functioned as reflexivity is often expressed as words of gratitude in the 
comments accompanying journal entries. However, some journal entries include information indicating that the authors are reflexive, especially in stating their interests in the topics being discussed, as well as some elements of their cultural values; however, they very rarely examine the assumptions on which positions, arguments and claims are made. Reflexivity tends to happen after-the-fact for most of the WPc community members. Interview respondents indicated a high level of reflexivity when asked about their engagements in discussions on the site. Although such information has value in and of themselves, they are often not consistently relayed back to the communal contexts from which they were extracted. By extension, people present themselves and are sometimes represented as 'subjects' of and for consumption, rather than being seen as an actively engaged civic person.

Emotions, and more generally affective states, responses and relations, are a critical, albeit rarely researched, aspect of political communication. Expressions of emotion, for instance, are frequently and often strongly manifested in the journal writings of WPc participants. The acknowledgement of emotionality, which is often seen and evaluated as the opposite of the rational, logical and disinterested thinking that is celebrated in Habermasian theory, can work to create space for WPc participants' reflexive practices. In a sense affective practices and relations are often the first step or stage in the development of interpersonal relations, and by extension of a relation of trust, between and across participants who are seemingly disconnected from one another by socio-cultural, political and economic differences. The disposition to take a reflexive attitude towards one's naturalized cultural values and world views is neither straightforward nor easily attained: the establishment of relations of mutual 
respect and trust between participants is a necessary step in this process, precisely because learning about and coming to understand the other is an important factor in denaturalizing one's own often 'universalized' world view.

Moreover considering that the majority of WPc members are women, the gender and identity dimension also plays a role in this process: it has been argued, for instance, that in a cross cultural sense women are generally less likely to disqualify or disparage displays of affect as an aspect of legitimate public discourse (see Carol Gilligan's discussion of gender in relation to the ethics of care, in Rachels and Rachels, 2011). On the WPc site, participants' contributions to discussions are shaped by both their individual and collective social experiences, and it is likely that "emotions, feelings and affect" will underpin these experiences (Dahlgren, 2014, p. 265). People's everyday lived experiences influence and shape their identity as communicative agents in political engagement online and offline: this is clearly the case with WPc, but equally it is not a notion that is integrated into Habermasian public sphere or communicative action theories.

Volkmer (2014) points out that "in the advanced stages of networked 'spatial' publics, civic identity is constantly being discursively re-negotiated and re-conceptualized, not so much with regard to a national or even a 'global' public, but rather across sets of 'publics of belonging"” (p. 189). In this context, the practice of engaging with and discussing diverse sets of issues binds the WPc community members to the practice of reflexive discourse, in the sense that the meanings, opinions, arguments, ideas and values that are expressed and articulated in these discussions are always going to be informed by a variety of cultural 
frames and positions. Moreover the strong cultural imperative on the WPc site to respect and engage with 'otherness', or at least with what most Western subjects would regard as forms of otherness, means that a level of reflexivity is already built into the culture and 'terms of engagement' that constitute WPc's version of the 'Gricean Cooperative Principle' that Bourdieu refers to (Bourdieu 2000, p. 122). In this very specialized sense reflexivity can be understood as having a place within public sphere activity on WPc. When certain assumptions are repeated, with no corresponding argumentation, analysis, evidence or reference to wider contexts, then their status as contributions to public sphere activity is clearly limited, certainly in a Habermasian sense, but also in terms of the 'rules of the game' operating on WPc.

The difficulty with Habermas' prescription about reflexivity is that it only rarely applies to or informs public sphere activity; this is particularly the case with the digital public sphere, where short, sharp and often 'shouted' comments tend to predominate, and where it is difficult to determine the level of cultural literacy of any comment, let alone its degree of reflexivity. Moreover, with the digital public sphere, the issue of the quality of any contribution (measured, for instance, with regard to its reflexivity, cultural literacy, and comprehension of the complexities of an issue) is often a secondary issue; what matters much more, in most cases, is the volume of contributions, the extent to which some of them constitute spectacular, dramatic or highly emotive discursive performances, or even whether or not the people providing them have significant levels of cultural capital, or are at least 'recognizable' to the public. 
Specifically on WPc, volume of contribution seems to be a major preoccupation with the site owners - arguably as it is also of major interest to other digital media sites whose worth and influence are measured by the number of clicks and comments they are able to attract to their postings. This need for clicks can be linked to my experience as a volunteer commentator on WPc. Volunteer commentators are recruited to make comments with no specific regard for the rational-critical or even the reflexive value of the comments made. Rather the emphasis is on how many comments are made overall on each post, which is indirectly influenced by the backend matching of commentators to journal entries. This stands in contrast to individual's self-directed posting and commenting on journal writings that they may consider of specific interest and deserving of a well-developed, relevant, engaging, and reflexive feedback.

Despite the problematical aspect of this practice of matching commentators with journal entries, excerpts from two of the three DACs quoted below show that some of the community members do bring a level of reflexivity to their submissions. Marvi82 provided a comment on japalouise's journal entry: "We are too far from your reality to give solutions but I think you should positively look at what you can offer and what you can give" (EGBV 51). EmmaKWin commented on chargerrose7's journal entry:

Thank you for sharing your story and experience. It is always inspiring to hear success stories, and I think yours is definitely one! You do a very good job of explaining your situation and solution in a way that can be expanded to other people (from the 
one to the many) which is critical to good journalism. Keep up the great work, Emma (GTTW 23).

Aminah responded to Nakinti's journal entry:

Very true Nakinti. It's not poverty that holds us back. It is ignorance. If we realize the importance of education we will strive harder to get it. If our parents realize the importance of it, they will starve but will send their children to school. If policy makers realize the importance of education, they will put on hold other issues but will prioritize education for all. We need to ensure education for all becomes top agendas of political leaders. That's the way for development. Thank you for being an inspiration to your sisters and also to your village people. Wishing you all the best (GTTW 25).

The interview participants' discussion of their practices on WPc further indicated varying degrees of reflexivity on individual levels that mirrors some of the practices observed in the journal entries and comments produced in the DACs. Reflecting on differences of opinion with other participants when engaging on WPc, Nkem wrote:

In life if anybody's opinion is different than mine I do not get angry or defensive. I believe their different opinion helps me understand the breadth of different cultures, 
traditions and human dignity in people. I would answer, "I find that interesting. Thank you for explaining your view to me." If I feel I have a different way to give them to look at things I do it with explanation in what I believe is a respectable manner (NR).

What we can take from these examples is that a culture that mandates 'respect for the other' simultaneously puts in place a reflexive mechanism that disqualifies, or at the very least discourages, participants from foreclosing positions simply because they are not part of the discursive doxa of the culture itself. To some extent the opposite is true: the position of the other must be respected and treated with respect, at least formally. There are two questions that arise from this reflexive mechanism. Firstly, is this mechanism capable of dealing or engaging with discourses that display no respect or tolerance for the other? Secondly, how can we decide whether this mechanism is merely performative in a formal rather than a practical sense? It is difficult to answer either question with any degree of confidence. If we take the exchange above as an example of this reflexive mechanism in action, what we seem to be dealing with is a reflex rather than a reflective response; in other words participants are more or less required to respond reflexively. We can only say that the 'of the moment' responses and discourses tend to be supportive, empathic and reflexive. However we can say, following Bourdieu's observation about the status of the gift (Bourdieu, 1999), that until those responses and exchanges are 'placed in time', their status is difficult to determine. 
WP staff's discussion of reflexivity indicated a reliance on two instruments for site management "1) community members' self-moderation that is guided by the site's prestated and publicly accessible terms of service, and 2) site management's proactive moderation and/or reactive moderation that is based on community members' report of inappropriate posts" (NR). The first instrument for site management seems to be the default used in most online forums, while the second instrument is becoming more commonplace as issues of online bullying, trolling, and flaming have become more of a public concern in online spaces. I could glean from the interview data that indeed WPc takes the issue of moderation seriously and expects its general community members to be a part of creating the safe space that it wants to be for deliberating on the issues, some of which are very sensitive for the people who have been affected directly. In her reflection, one of the interview respondents pointed out that:

In my experience on the World Pulse site, the differences among community members that most often require staff moderation have to do with either religion or sexual orientation. We address and resolve these differences by reminding participants of our core values: Voice, Respect, Generosity, Innovation, Connection, Courage, Solutions, and Accountability. We remind them that it is our goal at World Pulse for all comments and discussions in our online community to contribute to creating a welcoming environment for all. That we work to provide a safe and supportive space for members of all world regions, religions, and sexual 
orientations. And we ask them to keep the goal of using unifying language in mind in all of their contributions to the World Pulse community (WP staff 2).

\section{Empathetic Discourse and Engagement}

Ideal role taking is also required for well-developed public sphere deliberation. As Dahlberg writes: "Participants must attempt to understand the argument from the other's perspective. This requires a commitment to an ongoing dialogue with difference in which interlocutors respectfully listen to each other" (Dahlberg, 2001, p. 2). This characteristic is referred to as a form of 'ideal role taking'. It should be noted here that the criteria of reflexivity and ideal role taking are somewhat hard to separate from each other, as they sometimes intersect both in face-to-face forums and virtual space. Ideal role taking requires a respect for the contributions of others. There is certainly much respectful listening on WPc: it is more or less a requirement for anyone participating in communicative action. Perhaps similar to the observation made about exchange and critique, the nature of the site, as a forum set up primarily to give women an outlet for expressing their views on issues of common and public concern, sets up certain forms of address and notions of 'between us' that inflect contributions and exchanges.

This form of 'between us' can be gleaned from the journal writings and comments on WPc, but also from the exchanges occurring in other sections of the site, especially the resource exchange and VOF training sections. The 'between us' further extends to the offline 
interactions among and between WPc community members, as well as the website's management and its extended relationships with third party groups. In many of these diverse spaces, there is an extensive account and articulation of ideas, needs, knowledge, and other kinds of communal resources that represent WPc members as in-groups who are in the know, have certain exclusive privileges - like speaking rights at third party forums, representing the platform in rural communities and at public engagements, recommending new members for training and other community resources - and are fairly knowledgeable about the issues they discuss and the challenges facing their communities.

However, as Foucault would argue, a person cannot act in a manner that is free of external influences. Of course the dominant, popular ideology of and about the Internet is that it is a free space where subjects can exercise agency without recourse to the constraints of the offline world. Internet technology allows those engaging online to represent themselves in ways that are not limited by material or physical requirements of proof or authentication (for instance, a person who is female can quite easily take on and perform a male identity online), although the cultural influences and literacies derived from the offline world will continue to influence and inform online performances of identity in the context that participants can 'write' themselves into - and 'write back' through the commenting and journaling facilities. The narratives circulating on WPc provides a level of agency to the participants; as opposed to just being a consumer of the information, a certain level of agency that is undergirded by identity is attainable. 
Construction of identity occurs at the site of discourse (Poster, 2001; Turkle, 1995); people can take on whichever aspect of their identity they feel will serve their purpose at the time they are engaging in deliberations online. For instance, most people consider information about gender and ethnicity to be an essential component of online forum membership. Such is the case with WPc: community members generally want to know the 'true' identity of other members. Obtaining such information can be a challenge on some platforms, especially those that already allow anonymous posting, while other platforms will require authentication regarding 'true' and 'real' identity. A person's identity, validated or borrowed, can influence their ability to perform the criterion of 'ideal role taking'. This was evident in Nicole Staudinger's comment on Lubala Rosalie's journal entry about violence against old men in the Democratic Republic of Congo:

I admire your empathy and care in bringing attention to the plight of the elderly. It is especially sad that children would abandon their parents after being cared for their entire lives. I agree that more should be done to assist the elderly, and to have them be made to feel valued (EGBV 11).

Gbemi Abeow commented on Adele Wasolu's journal entry about a fight in Kamulia and Chilaji, the Eastern region of the Democratic Republic of Congo, in which six deaths were reported and several people injured. Gbemi demonstrates a level of ideal role taking when she draws on her own personal experience of fear and concern about her personal safety during a conflict in Jos, Nigeria: 
Dear Adele, I understand how it feels when your safety and that of your family is being threatened. I was at Jos, a state in my country in Nigeria when fightings and riots broke out and I had fears that it would get to where I was. I was trapped were I was, I couldn't even finish what took me to Jos in the first place. However, I was very lucky to find a transport company that was going on its last trip back to Lagos from Jos, two days into the riots in which people were killed and many injured. Indeed Adele, I understand; and I can imagine that the situation must be a hard one to bear. Writing this makes me see that you are strong. And I pray with you and for you and for your family and all your loved ones that you will remain safe and saved. I also hope and pray that the fighting in Congo will stop and that peace be restored to your country. You will always be in my prayers (EGBV 31).

Maya Norton's comment on chargerrose 7's journal entry in the GTTW digital action campaign, in which the latter discusses what can be done to break down education barriers in America, is also relevant here:

ChargerRose, your first journal entry hurt my heart. Have you found people, especially women, to connect to since your first 
writing? Depression is never ever the individual's fault. You are

brave to write about it and ask for support and help. It sounds

like you are Mormon since you mentioned missions, right?

(GTTW 23).

Also relevant is Gracest's comment on Garima's journal entry about the potential of African countries to use technology to combat poverty and achieve sustainable economic growth:

You bring up great points about technology and its potential to empower African citizens. I especially appreciate your point about the nation losing many of its scientists and higher-ed graduates to other countries. The key is to make Africa a welcome place for these intellectuals to establish themselves at home. I hope that m-learning offers that opportunity to create a breeding ground for innovation from the ground up (WWTW 12).

These exchanges demonstrate, at the very least, that the performance of ideal role taking is implicitly prescribed, without ever being formally constituted as a rule of exchange. Ideal role taking is evident and consistently appears in almost all the journal entries, and particularly in the accompanying comments. Most of the WPc community members tend to both implicitly and explicitly draw on their own personal experiences and connections to 
the main issue on the agenda, as well as the additional issues that emerge in the course of the discussions. Interview respondents point to this practice of ideal role taking as well, when recounting their experiences about participating in the digital action campaigns on WPc. There is little evidence of difference of opinion. However, to some extent this forecloses the possibility of a rigorous and extended dialogue that would enrich the deliberation and allow for more robust discussions. The interview participants expressed these expectations as an extension of the discussion of reflexivity. Nkem said "yes, I absolutely will change my opinion based on something a person told me on WP and if I can have time to verify this is credible" (NR). Emeka said "maybe Yes or No, It depends on my final conviction/decision about the discussion and how it can be proved with other criteria" (NR). Ifeoma said "off course, if the person has a valid point backed with critical statement, why not!" (NR).

Some interview participants indicated that they would change their opinion on a specific issue they are discussing on WPc if another participant has a superior and verifiable argument. The superiority of opinion and verifiability of the facts being presented was important to the interview respondents, although there is no articulation of how this verifiability is to be achieved. Presumably, individuals would verify the information afterthe-fact or they rely on WPc management for verification of information shared on the website.

'Natural' Sincerity 
Criterion five requires that "Each participant must make a sincere effort to make known all information - including their true intentions, interests, needs, and desires - as relevant to the particular problem under consideration" (Dahlberg, 2001, p. 2). Sincerity is one of the factors that Habermas identifies as being both essential to public sphere discussion, and a key marker of civic engagement. However, while the sincerity or authenticity of participants in public sphere exchanges may seem a relatively complex proposition in terms of conventional and historical public sphere activity, it becomes even more problematical once digital media is taken into account. This is not just because in pre-digital contexts and situations it was much easier to authenticate the status and identity of participants; after all, even if a person was to publicly declare for a position 'in person', that need not attest to or prove their sincerity. Sincerity and authenticity have always been (necessarily) determined or evaluated by way of a set of performatives. In other words, to some extent the question of the sincerity or authenticity of a person's contribution was predicated on the person meeting certain performative requirements, such as using simple or straightforward language, being reflexive about their own position, or making use of certain authorized discourses (performing community identification, empathy or a commitment to core values). Of course, the criteria for determining sincerity change according to time and place; in some circumstances, one overriding discursive performance might be required (nationalism, patriotism or opposition to terrorism), while on other occasions the criteria might be more formal (plain speaking, displaying sympathy or emotion).

Hence we can argue that the expectation of sincerity is a performative in the Butlerian sense of the word, and not necessarily a 'real' need for a platform to function as a public 
sphere. Expectations as to what counts as or constitutes a legitimate performance of sincerity will vary from one culture to another. What a community of people come to see as 'real' or 'true' is based on the discourses in circulation in their offline spaces, and further influenced by the discourses in circulation on WPc. Volkmer argue that 'identity' and 'reflection' are key elements of deliberation in virtual public spheres; participants can represent national interests and still indicate a stake in global and transnational issues as well (2014). In the age of new media, participants use many tools to signal sincerity and also to engage at more personal levels. These forms of sincerity may not necessarily be the same kinds, as Habermas would expect. Moreover economic, demographic and cultural transformations occurring worldwide affect how much sincerity participants can display on a forum, especially when it is a global space (Ugarteche, 2007; Volkmer, 2014). Most people still want to represent their own national interests while simultaneously acknowledging that some of the issues they are discussing have global consequences; for instance international issues have as much significance as national issues for the participants in Volkmer's (2014) study that included people from different regions of the world.

With the digital public sphere, two sets of problems arise that make the issue of sincerity problematical and difficult to evaluate. Firstly, and unlike the case with the pre-digital public sphere, there is often no obvious or continuous connection between the (digital) identity of the participant and the position taken or discourse utilized. Secondly, whereas in the pre-digital public sphere there was usually a more or less accepted set of criteria for evaluating the sincerity of a participant, this is not so much the case with the digital public 
sphere. However, the criterion of sincerity is a common feature in WPc discussions: almost every journal entry and comment contains a "declaration of interest". However, "true intentions" are hard to judge. Beverly Rose's comment on tressa kentner's journal entry in the EGBV campaign is a case in point:

Thank you for sharing this story. I have been a sexual/domestic violence advocate for over 10 years on this tiny island 'paradise' in Hawaii. I can relate to the shortcomings of the judicial system all too well, unfortunately. I agree with you regarding education as being key, education and economic empowerment (EGBV 2).

The sub-criterion of sincerity that is in play here, most obviously, is empathy: the contribution of the responding participant establishes a connection by way of a declaration of thanks and identification. However, what is also interesting about this passage is the way in which it foregrounds its performance of sincerity and authenticity; it starts by thanking the poster for their contribution, then cites their own activities in the area as a marker of their connection with the post and poster, empathizes with regard to their further points, and generally agrees with the tenor and scope of the post. What is significant here is not so much that the respondent agrees with the sentiments or arguments of the original, as much as the commitment to the importance of the issues and causes under discussion, and the discursive performance of community and connectivity (shared experiences, knowledge, activities). The three criteria of sincerity, reflexivity and ideal role taking in Habermas' theory of rational communication are interconnected. Interestingly, the manifestation of 
these three prescriptions on WPc is strongly influenced by (and overwhelmingly manifested through) various degrees of emotionality expressed by WPc participants. Emotionality is an essential and unavoidable feature of communication practices that Habermas, and many more theorists of public and political communication, omit from a conceptualization of the public sphere and communicative action. Elements of each criterion can be seen in the other in the ways they manifest in deliberations on WPc. This observation is similar to the one made in another study of a news forum in which the analysis focused on political campaigns and discussions during a federal election in Canada (Isekeije, 2012).

Exclusive Inclusivity

Discursive inclusion and equality constitute the sixth criterion of the public sphere. Dahlberg writes that:

Every participant affected by the validity claims under consideration is equally entitled to introduce and question any assertion whatsoever. Inclusion can be limited by inequalities from outside of discourse - by formal or informal restrictions to access. It can also be limited by inequalities within discourse, where some dominate discourse and others struggle to get their voices heard (Dahlberg, 2001, p. 2).

The WPc community presents itself as a diverse yet inclusive environment that encourages the expression of views from its cross-section of members. This presentation of absolute inclusivity - a culture to which there is no trace of dissent or opposition - suggests a 
utopian virtual space that is uncommon in online forums. Dissent is conspicuously absent on WPc; there is an almost utopian culture that encourages, and almost demands, that participants support each other. The website encourages individual journal writers and commentators to make contributions, with minimal monopoly of discussion from any specific group. One of the interview responses indicates how this environment is created and maintained through moderation by community members, community volunteers, and staff. Two World Pulse staff members are specifically tasked with assessing new content published by community members on the platform. WP staff 2 wrote that, "There are instances, though, when we will have to unpublish content and notify the member that that type of content is not permissible on our site. When this happens it is usually hate speech" (NR).

Unequal positions of participants and power relations between participants influence engagement and experiences in the public sphere. Marx draws our attention to how class and social relations influence our experiences and understanding of the world. Factors such as economic and cultural capital, language skills and technological access and knowledge limit the possibilities of inclusion in the deliberation and further makes equality difficult. Moreover, discourses determine how we experience, order and evaluate the world; the experiences of WPc participants both on the website and offline is necessarily inflected by the ongoing discourses in their everyday lived realities and what they are exposed to in their work, school or even more broadly, the media. Reader comments and blogging skills are advantages of new media that are gradually creating space for inclusion in the public sphere (Volkmer, 2014) although national interests are still prioritized over global or 
transnational agenda even in those comments and blogs (Ugarteche, 2007). This is mitigated, to some extent, on the WPc site where these factors are at least theoretically taken into consideration.

Moreover not everyone has an opportunity to introduce a topic on WPc, although the format of writing journal entries and extended comments allows community members to include new ideas and expand on the initial topic the WP management introduced. However, in placing a focus on specific countries and regions during certain campaigns - the EGBV focused on the Democratic Republic of Congo while the GTTW focused on South-East Asia - WP management invariably 'directed' where most of the contributions came from during the two campaigns. Despite this, comments in the journal entries still came from a diverse group of people in different parts of the world. Some of the ways that discursive inclusion and equality manifests on WPc include the opening statement by WP management on each page of the Ending Gender-Based Violence 2012 campaign:

World Pulse channeled your voices to major media outlets, advocacy groups, and policy makers to ensure that your perspectives were heard in the halls of power (EGBV 1).

Hannah Cynthia commented on Tessie's journal entry that attempts to disrupt the discourse of women as victims of violence, by suggesting that sometimes women are implicated in the violence that they experience. In her post, Tessie suggests that certain personal traits displayed by women predispose them to violence from the opposite gender, and perhaps 
these traits also make some women accomplices in the violence that other women experience:

As women we should aim to bring about change that promotes equality and respect for everyone (EGBV 1).

\section{SEE THE PRINT VERSION FOR ACCESS TO THIS CONTENT}

Loyce illustrates her journal entry titled 'Women Confront VAW using ICTs: Experiences From Remote and Fishing Community in Eastern Uganda' during the 'Women Weave the Web 2014' campaign.

Estelle's comments on Abby Kakiyes' journal entry about the leadership challenge in Nigeria in the face of the Boko Haram crisis, and how the failure in leadership is disrupting education for young children and youth in the country, is relevant here: 
I know its been tough in Nigeria since the rise of B,HARAM.

But $\mathrm{i}$ want $\mathrm{o}$ appreciate $\mathrm{u}$ for the project $\mathrm{u}$ are carrying out in ur community, great job. we are all activist for women and girls around the world. thanks for sharing the realities in ur ommunity (GTTW 1).

Torilynnfox comments on Gloriabit's journal entry recounting the appalling living conditions of girls in mining communities in the Democratic Republic of Congo. Some of the issues that Gloriabit identified are forced early marriage, prostitution, teen pregnancy, rape, and husbands pimping their young brides as sex slaves to miners. Both genders are implicated in this practice, but men more so than women. There is also the point of the privileging of male children over females:

I know it must be emotionally exhausting at times to recall all the troubles women in your community face. But speaking out like that can only bring positive change. Your courage and strength will be so influential and inspirational to other girls in your community and all over the world (GTTW 22).

Susa comments on Mauri's journal entry about the challenges of navigating online web spaces in the quest for digital literacy and having a good working knowledge of technology. Mauri talks about social, cultural and economic challenges of software acquisition that she 
and her peers had to overcome, as well as the broader hardware technology issues that were mostly out of their control:

I was also especially interested in your comment about getting beyond digital literacy as a communicative tool (as essential as that is) and to entering the realm of computer programming. Perhaps this is an aspect of digital literacy that can get more attention in the future and can attract more women to the opportunities it affords (WWTW 31).

Zoneziwoh comments on Olutosin's journal entry about the latter's transition from a solitary experience of writing with no audience to the joy of finding kindred spirits on WPc. Olutosin recounts that her husband introduced her to WPc, and since she joined the community, she has seen her ideas take better shape and she has been able to access many opportunities she otherwise thought were impossible because of her location and station in life:

What else can I say. I am drown in emotions by your words, as I see myself in you: with no 'god' father 'god' mother but here at WP, we are one another's 'god' mothers/aunties. Here we have redefine the concept of god parents (WWTW 55). 
A key voluntary role and the work done in the position of Community Welcomers helps to foster the perception of WPc as a space that is inclusive. This reflects a dimension of discursive inclusion and by extension opens up opportunities for equality vis-à-vis encouraging all members to contribute to the discussions. However the format of inclusion that is employed by WPc - which directs and delimits the types of contributions, journal writings and commenting that community members engage in - presents the platform as an exclusive space that privileges certain forms of address and discourse (that reflects liberal, left-leaning, socialist disposition to the issues being discussed). This format of inclusion indirectly positions WPc as an exclusive space that has no place for women with conservative, right-leaning views.

\section{Conclusion}

The deeply rational reflection demanded by Habermas did not characterize the digital action campaigns that were ran on WPc. Instead contributions were often supportive, thoughtful, understanding and sometimes emotional. Discourses of supportiveness, empathy and emotionality are considered legitimate within the culture of WPc, which is committed to the objective of representing women and amplifying their voices, and facilitating discussions about the issues that these women care about. The performance of 'ideal role taking' is commonplace on WPc, as most participants' identity is verified: hence their expression of empathy can be related back to the multiple ways they have represented themselves on the platform. Community members often draw on their own personal life stories to identify with the issues that other community members bring up for discussion. 
Sincerity is a problematical concept in this context, and arguably something that is impossible to identify in public sphere deliberations, especially in digital public sphere where people have opportunity to take on and perform multiple identities across many platforms, and sometimes even within one platform. Inequality and lack of inclusivity are still commonly experienced in public sphere sites, even with advancements in digital technology. Class and social relations, as well as the ways in which language and discourse problematizes opportunity for inclusion, continue to limit access for people with limited socio-cultural, economic and political capital. Digital media's interactive format is however assisting in reducing these inequality and exclusionary practices.

Habermas' public sphere is similar to Bourdieu's 'Gricean co-operative principle' that allows us to assess activities and events based on the ideals for those activities and events; for example, a conversation about healthcare should be considered a dialogue if it has addressed and articulated healthcare issues that are related to the discourse of health. Both public sphere and Gricean co-operative principle are ideals that society should be working towards. As seen in Lunt and Livingstone's (2013) argument, there is a clear indication that suggests WPc can be assessed as one of the possible public spheres in which contested communication occurs. WPc claims to be representing women and bringing women a global voice in the public domain; it represents itself as a global space for women to engage, interact and discuss issues of commonality to women regardless of where they live. This to me suggests that WPc purports to be a digital public sphere. 
It is against this background that I chose to assess the work the website is doing using Habermas' yardstick for determining if a space indeed functions as a public sphere. Using Foucault to address the shortcomings found in Habermas' theories, most of which have been pointed out by other scholars, was helpful in ensuring that there was not a blanket, dogmatic yardstick used in assessing WPc as a public sphere site. The work of the site and the experiences of its members constitute a collection of discourses, activities and practices that are being done both offline and online to advance women's claims to be included within what we might term the universalization of universal rights. In that sense the website clearly functions as a micro public sphere.

Dahlberg identifies six criteria as being necessary and continuous with regard to the Habermasian notion of the public sphere: these could be described, in a general way, as 'autonomy', 'exchange and critique of criticizable moral-practical validity claims', 'reflexivity', 'ideal role taking', 'sincerity' and 'discursive inclusion and equality'. Matching these prescriptions against the communication practices and contributions of members of WPc, and more generally of the WPc site considered as both an online community and an institutional culture, provided an understanding of the extent to which WPc was able to meet its own aims and expectations; however, and perhaps more importantly, it also tested and provided insight into the value, applicability and practicality of both Dahlberg's six criteria, and by extension the Habermasian notion of the public sphere.

In the following chapter I will extend this process, specifically in terms of focusing on issues of digital divide and digital inequality. I will do this by way of an analysis and 
evaluation of the data collected through the DACs, online web survey and email interviews, in order to focus the analysis on the five broad forms of digital inequality identified by DiMaggio and Hargittai. 
Chapter Seven: Digital Inequality, Public Sphere Activity, and WorldPulse.com

Introduction

In this chapter, I use the data collected through the DACs, online web survey and email interviews to focus the analysis on the five broad forms of digital inequality identified by DiMaggio and Hargittai. This analysis will assess the interactions among participants on the WPc platform; some of the various groups the participants work with; issues of inequality, either as identified by the participants or as observed in their interactions; and some institutional aspects of digital inequality. Indicators of digital inequality include inadequate skills and knowledge about the issues being discussed. Institutional inequality could also be due to interactions among the strategic choices of corporate firms, consumers' responses, and government policies. Indicators of institutional inequality include references to inadequacies in Internet technology, a lack of digital access, and the use of ICT by WPc community members' organizations and WP management.

This assessment of DiMaggio and Hargittai's digital inequality model - which they have operationalized as five broad forms of inequality - simultaneously analyzes the practices and culture of WPc as a digital online community as well as the socio-cultural, political and economic practices of WPc management and members in their various offline communities. The assessment is organized as: 1) the persistent digital divide - including a specific focus on issues of digital divide online and offline; 2) increasing digital inequality - including digital inequality among individuals and groups, institutional digital inequality; 3) digital 
capacity building - digital literacy, individual capacity building, team based capacity building, institutional capacity building, language; 4) socio-cultural inclusivity - digital inclusion, support; and 5) variable digital affordances - including a specific focus on digital affordances, digital empowerment for individuals or groups, and institutional digital empowerment.

Five Broad Forms of Inequality

DiMaggio and Hargittai's claims about the five broad forms of inequality are analyzed, evaluated and critiqued through an assessment of archived journal entries I collected on the WPc site, as well as through the online web survey and email interviews. It should be pointed out that DiMaggio and Hargittai's questions about the influences of corporations and government institutions on issues such as the digital divide and digital inequality are outside the scope of this research. Addressing these institutional factors would require gathering data from the institutions and corporations. However, in instances where WPc community members make references to what institutions are doing to alleviate or aggravate digital inequality, I include such information in the assessment.

The Persistent Digital Divide

The first form of inequality examines technical apparatus. DiMaggio and Hargittai ask, "How does inequality in the adequacy of hardware, software, and connections limit the ways in which different kinds of users can employ the Internet?” (2001, p. 9). They infer 
that, "inferior technical apparatus reduces the benefits users can gain from the Internet directly or indirectly" (p. 9). Access to technological facilities in most spheres of activity is still likely to be predicated or dependent upon levels of financial or cultural capital. By way of example in the case of faculty at a university in Serbia, the kind of digital device a faculty member has access to depend on their status as a junior or senior faculty member. The digital devices have different kinds of applications and a faculty member's ability to use the device determines whether the technology can be used for facilitating learning in the classroom (Radovanovic, 2013). In this context, it is virtually impossible to avoid the distinction of unequal access to and use of technology. It is apparent that inequality is built into the form and dispatch of the technology of the Internet.

Volkmer (2014) draws our attention to the fact that "different generational media and communicative cultures manifest in public engagement" (p. 182). The scholarship on the interconnection and intersection of new media technologies and social inequalities suggest that the digital divide is an aspect of social inequality. Ragnedda and Muschert (2013) argue that "differences in digital proficiencies create new inequalities" (p. 2). However we also know that data-enabled mobile phones afford activists the opportunity to engage in civic activities while they are in transit, and eliminate the limitation of having to be connected by wires when using a computer; portability is a major advantageous feature of mobile phones when contrasted to trying to do the same work on a tablet or laptop. As Gordon has shown, this was seen to be the case in the use of mobile phones in the Philippines for political purposes (Gordon, 2001). To assess DiMaggio and Hargittai's 
claim about digital inequality, I examined the data from the digital action campaigns, the email interviews, and online web survey using the code 'digital divide'.

\section{Digital Divide}

This code was used to capture WPc community members' discussion of challenges with regard to access to (and in some cases, the use) of new technologies during the DACs. This discussion is quite frequent across all three campaigns, but especially in the WWTW campaign, which focused on the use of digital technology for communication and community development.

Kit's comment on Y's journal entry about her experiences in the Appalachians is relevant here:

Your journal post illuminates the mixed blessings of the internet as well as the differences in our expectations regarding connectivity, which in part, arise from where and how we live. I am sensing that there is a considerable amount of adjusting and adapting taking place in Coker Creek. Some are becoming used to the marvelous portal that has thrown open the world, while others, such as yourself, are dealing with issues of less immediate connectivity (WWTW 52). 


\section{SEE THE PRINT VERSION FOR ACCESS TO THIS CONTENT}

Image illustrating Y's journal entry about Internet access in the Appalachians for the 'Women Weave the Web 2014' digital action campaign.

'Women on the rising's' journal entry in the 'Women Weave the Web 2014' campaign talks about the gender digital divide that is traceable to the discourse of women's place in the domestic or private sphere. Her submission is focused on some of what she experienced in Owerri, Nigeria. Rasheedat Sanni uses her experience of accessing the Internet at a cybercafé to discuss issues of privacy, digital access, safety and the intersection of online/offline empowerment:

Privacy: whenever I have to go to the cyber cafe, a feeling of repulse goes through me,this is because the place is seen as a male hangout or workplace and in truth,you hardly find any woman there and if one comes around,she probably brings her 
ward to the operator who helps them with the operations while they look on, the phobia on how to operate it and distancing from it is just there,coupled with the cold looks and stares and inability to operate certain functions kill the zeal in women with their conservative nature; they are rather shy and hence prefer not to go further but thanks to the smart phone which helped break this barring ice and makes it easy for the majority of women to access the internet practising on their own indoor or at their business post with or without cordial assistance (WWTW 9).

Kkibaara's journal entry about the differences between her and her sister in their approach to digital technology attributes some of these challenges to women's mindset about technology as being more for men and younger people, but also to the fact that fast paced changes mean that it is hard for the older generation to keep up with new technology. A lack of electricity and the cost of access, especially when using a cybercafé, are some of the other issues she identified specifically with regard to her home country of Kenya:

Power outages, especially if the computer the woman is using is not connected to a UPS to give her a few minutes to complete what she was doing is a challenge that affects women especially in the developing counties. I have heard that there are countries especially in Africa that have constant power outages and yet the 
digital technology is powered mostly by electricity. So if there are constant power outages, it means even if they have smart phones, once the battery power is gone, they will only be able to get online once power is back (WWTW 34).

Nabiye Tal's journal entry outlines the challenges women face accessing the Internet in Nigeria. Some of the issues she identifies include inadequate infrastructure, social, cultural, and economic barriers, rural-urban divide and gender gaps:

From my experience in working with women through my NGO, Initiative for the Elimination of Violence against Women and Children (IEVAWC), I see that Lack of financial resources is what hinders women to participate fully in the web. This is because almost all communication facilities cost money and majority of women are afflicted by poverty.

Also as a women's right activist, I realized that to achieve the right to communicate as a basic human right for women in Nigeria, women have to take on themselves the difficult task of gaining access to the necessary infrastructures that will enable them use the internet. This entails the provision of more infrastructures that will take care of the majority of women who reside in rural areas as well as making available the latest infrastructure for users' needs (WWTW 45) 
Tables 20 and 21 show information from the survey data indicating that the location of WPc community members cannot be correlated to their participation in the digital action campaigns and the 'Voices of Our Future' training program. More detailed information from the survey is available in Appendix 3. 
Questions 4 and 14

Which region of the world do you live in? * Have you participated in any of the Digital Action Campaigns on WorldPulse.com?

\begin{tabular}{|c|c|c|c|}
\hline \multirow{2}{*}{$\begin{array}{l}\text { Which region of the world } \\
\text { do you live in? }\end{array}$} & \multicolumn{2}{|c|}{$\begin{array}{c}\text { Have you participated in any of the } \\
\text { Digital Action Campaigns on } \\
\text { WorldPulse.com? }\end{array}$} & \multirow[b]{2}{*}{ Total } \\
\hline & Yes & No & \\
\hline North America & 4 & 2 & 6 \\
\hline Africa & 7 & 7 & 14 \\
\hline Other & 6 & 2 & 8 \\
\hline Total & 17 & 11 & 28 \\
\hline
\end{tabular}

Table 20: Cross-tabulation of survey respondents' location and digital action campaign participation.

Questions 4 and 18

Which region of the world do you live in? * Have you taken the Voices of Our Future training on WorldPulse.com?

\begin{tabular}{|c|c|c|c|}
\hline \multirow{2}{*}{$\begin{array}{l}\text { Which region of the world } \\
\text { do you live in? }\end{array}$} & \multicolumn{2}{|c|}{$\begin{array}{l}\text { Have you taken the Voices of Our } \\
\text { Future training on WorldPulse.com? }\end{array}$} & \multirow[b]{2}{*}{ Total } \\
\hline & Yes & No & \\
\hline North America & 1 & 6 & 7 \\
\hline Africa & 10 & 8 & 18 \\
\hline Other & 10 & 3 & 13 \\
\hline Total & 21 & 17 & 38 \\
\hline
\end{tabular}

Table 21: Cross-tabulation of survey respondents' location and Voices of Our Future training participation.

Tables 20 and 21 show that there is no connection between the region of the world participants live in and the level of participation in both the digital action campaigns and the voices of our future training program. If one is to go by the assumption and stereotype that living in urban rather than a rural area, or a developed rather than a developing country, affects the quality of technical apparatuses, this does not seem to appear to be the case in the responses to the survey data. The tables indicate that other factors beside 
location affect respondents' participation in the digital action campaigns and the 'Voices of Our Future' training.

Interview participants own multiple technological devices and have exclusive use of their devices, except for one participant who shares her desktop computer with other people. However, there is diversity in the experiences of the participants in their use of these devices, depending on the type of device and point of Internet access. Emeka writes that "Using a tablet makes it handy to access the internet (worldpulse inclusive) anyway there is internet service compared to carrying laptop everywhere" (NR). For Uloma "WorldPulse is easy to access on all devices, especially since they developed a mobile platform, so that it more how I noticed the experience on the device. Mobile devices make it easier for me to check into internet sites more often" (NR). Amara writes that "With the use of mobile phone, my participation on WorldPulse has reduced to may be 10percent. I hardly write except when it is absolutely necessary because my mobile phone has low memory" (NR). Ogechi said "I use the mobile phone from time to time to view emails that have come in when I am not connected via my laptop. But I use my laptop for most all correspondence" (NR). Ifeoma also makes a contribution "I have a netbook and sometimes it gets hang and typing lot of stuff at a stretch is little bothersome. But, overall it is fine as long as I am able to do my work and read stuff and update the posts on WorldPulse. As such, I don't face any problem in accessing any information from the internet and specifically from Worldpulse. I enjoy using my device in general" (NR). 
From the three data sets obtained through archived journal entries, web survey and email interviews, it is obvious that the digital divide persists in both the Northern and Southern hemisphere. Through their journal writings and information supplied in the survey and interview responses, WPc community members identified and discussed specific factors that are contributing to this persistent digital divide. These factors include the ongoing perception of women's place as being primarily in the domestic sphere, the perception of technology as masculine, inadequate technological infrastructure (which is manifested in the lack of electricity in some communities), unstable Internet access, socio-cultural and economic barriers, rural-urban digital divide, gender gaps, concern about privacy, security and safety when accessing the Internet in a public space, fast-paced changes in both hardware and software technology, and the very significant cost associated with access to technological devices and the Internet.

Increasing Digital Inequality

Economic and cultural capital can influence or determine the autonomy of use of technical apparatus for most people engaging in the public sphere. A lack of political capital can further compound the challenges of access for those engaging online for the purpose of civic engagement (Wellman et al., 2001). Even though Internet use is considered, in terms of political activity, to be supplementary to face-to-face and telephone communication, a person's ability to use the device independently can affect what they are able to do on and with the device. For instance, when looking at traditional media, the one-way letter writing practices of two $19^{\text {th }}$ century American women as assessed by Gring-Pemble (1998) shows 
it was an autonomous activity that allowed the women to connect what was happening in their two independent private homes into a form of public engagement that would later influence the early feminist movement. The use of journals as a site for raising consciousness about issues of concern to these women is very much similar to the electronic journals and the accompanying comments produced on WPc by its community members.

With regard to this inequality in autonomy of use, DiMaggio and Hargittai raise the question, "How much control do people exercise over their Web use? Among persons with Internet access, are there significant intergroup differences in the extent to which that access is at home, as opposed to work, school, libraries, or other community access centres?" (2001, p. 9). They suggest that "Among people with access to the Internet, the greater the autonomy of use, the greater the benefits the user derives" (p. 9). In assessing the archived journals on WPc and the interview data, two scenarios of autonomy of use were observed. These are: 1) digital inequality with regard to individuals and groups, and 2) digital inequality at an institutionalized level. These codes emerged while examining the discussions, accounts and indications of the experiences of individuals and groups with regard to autonomy of use, as reported in the journal entries and comments in the digital action campaigns, as well as responses to interview questions. Interview participants give accounts of getting more benefit from using the Internet because: i) they own their electronic devices for accessing the Internet, ii) they own multiple electronic devices, iii) they can access the Internet from their preferred location, and iv) they can access the Internet at their preferred time. Chika writes: "Of course owning a device is an advantage. 
It allows me to access the internet flexibly, the time and location I want, as long as I am connected to the network" (NR). For Uloma "WorldPulse is easy to access on all devices, especially since they developed a mobile platform, so that it more how I noticed the experience on the device. Mobile devices make it easier for me to check into internet sites more often" (NR). Uloma's observation about WPc's introduction of a mobile version of its website further buttresses the additional ease of accessibility - which is intricately linked to autonomy of use - that the site affords its community members. However, this autonomy of use does not equate to the same degree of autonomy in relation to Habermas' expectation that participants and sites of discussion should be autonomous from political state establishments and economic power.

SEE THE PRINT VERSION FOR ACCESS TO THIS CONTENT

Paola's journal entry titled 'Our History Has Focused on the Spirit of Discovery in Women,' submitted to the 'Women Weave the Web 2014' digital action campaign. 
Digital Inequality - Individual and Groups

When Liff and Shepperd assessed the take-up of access to the Internet in British households, the survey data showed that "the relationship between experience, use and confidence is gendered" in favour of men $(2003$, p. 5). There is also evidence of issues of inequality in skill in relation to education, an observation that confirms DiMaggio and Hargittai's position that autonomy of use can affect how one engages with Internet technology. Contextual analysis of the quantitative data from the Oxford Internet Survey indicates that gender divide persists mostly in terms of technological access as well as the skill access barrier (Liff and Shepperd, 2003). Emden and Midgley posits that, "a working public sphere requires knowledge about what is relevant to the public, that is, it requires the kind of expertise - about science, about international relations, even about tax law - that most citizens simply do not possess" (2013, p. 7). Emden and Midgley’s position relates to Liff and Shepperd's (2013) as well as Tully's (2013) discussion about soft skills beyond technological access, that most participating in public sphere deliberations need in order to be effective for their communities and the global sphere. With regards to digital inequality across individuals and groups on WPc, Moshelbeads' comments in her journal entry about her experience using mobile phones to access the Internet, and assisting other women in Ghana is relevant. She also refers to the need for governments and civil societies to assist people who need soft skills training. This was a response to a comment by Busayo in which the latter provided links to the work her organization is doing to empower women in Nigeria, including establishing a cybercafé exclusively for women and girls: 
I just checked what your organization is doing for women. The Cybercafe for Women and Girls Only initiative by your organization is laudable. I was introduced to the internet at 9 years old (in my school in Ghana) and my big sister always encouraged me to use the internet. However as a teenager I wasn't comfortable going to cybercafes because of privacy issues, once a guy sitting by me suppose to be using his computer sent me a chat message that "he wants to be my friend" and that he is the one sitting by me. I got scared and left, since then when going to a cybercafe I look out for the ones with table dividers. Thanks for what your organization is doing (WWTW 17).

Stella Paul's comment on her own journal entry details her use of mobile phones to assist women in rural India to share their stories with the world. Stella was able to train Jyoti on how to use SMS with Hindi and Roman script for a 140-character message to produce a news report:

The biggest challenge before us (I mean, you, me, Loice \{who's commented below $\}$ is that we are dealing with a community of customers with little purchasing power. So, it has to be a cheap handset and the apps should not require an internet data package. In my case, the handset is basic and the user has to register her 
number with a website just this once. After that, she can keep using the apps without paying anything. The registration requires renewal once in a while - like every 3 months which is quite doable (WWTW 58).

These women's accounts of their experiences with the use of digital devices, along with the use of Internet platforms and applications to engage within their local communities and express solidarity with other women in global communities, indicate the importance of socio-economic, cultural and political capital as proposed by Bourdieu. Through these discussions, we can also glean that there are consequences for women who are limited by inadequate skill to use the devices - as these affect their independence and the preferred autonomy that should undergird their public sphere participation. It is difficult to derive so much benefit and explore the advantages of digital technology if a citizen's autonomy of use is limited or compromised due to a lack of technical knowledge and soft skills.

Digital Inequality - Institutional

With regards to institutional digital inequality, Martha Llano's comments are relevant here. In her own journal entry, she uses the analogy of running water to illustrate the importance of digital access and empowerment for local women in Colombia:

Everything is controlled. And although my department is one of the most educated and open about the use of technology and 
there is free access to almost all the heads of our municipalities is still very sad to see that the rest of the municipalities not and rural areas ... Internet is the right to information, build democracy and should be right where our food comes .... as I see ... our rural people live where they come from our food and pure drinking water ... why not reinvest in them? somehow give back what they do? (WWTW 46).

Olutosin's journal entry recounts her experience going from a lone person writing about life in solitude to an empowered woman who can now access multiple opportunities to share her ideas with the world through her connection with the WPc community. She highlights the need for government institutions to take a leadership role in ensuring Internet access for the citizens:

The duty of a good government is to ensure a safer community for the citizens, Festac town is notorious for cyber crime, therefore it is unsafe for women to surf the net in the public cyber café, reducing the cost of internet will make it affordable to all and improvement in electricity supply is a must (WWTW $55)$.

A common theme among these two journal entries is the assumption that support from government agencies and civil society organizations can further assist citizens in their need 
for digital access and appropriation of digital technology devices and platforms. For Martha especially, digital access correlates to empowerment for women in Columbia. Olutosin extended the discussion to highlight the failures of government institutions to provide security in public spaces where citizens access the Internet. This is a common and recurring theme among most of the WPc participants from the Southern hemisphere who contributed to the DACs - they would all like government to take a leadership role in ensuring safe and secure access points by improving electricity supply, making connectivity affordable and providing hardware improvements.

\section{Digital Capacity Building}

Skill is an essential ingredient for civic engagement in online public spheres. Forms of knowledge and literacy, both technological and cultural, are necessary for effective civic engagement. There are new literacy requirements for people to be able to take advantage of new technological tools (Radovanovic, 2013). For instance the importance of education is apparent when one looks at the data from the online web survey of this research. Giddens (2006) considers education an important prerequisite for effective and sustained civic engagement. The survey data indicate that all the respondents have some level of formal education (one respondent did not provide this information), with more than half of the respondents having a postsecondary level of education. 
Question 5: What is your highest level of education?

\begin{tabular}{|c|c|c|c|c|c|}
\hline & & Frequency & Percent & $\begin{array}{c}\text { Valid } \\
\text { Percent }\end{array}$ & $\begin{array}{c}\text { Cumulative } \\
\text { Percent }\end{array}$ \\
\hline \multirow[t]{6}{*}{ Valid } & Bachelors & 19 & 40.4 & 40.4 & 40.4 \\
\hline & Postgrad & 24 & 51.1 & 51.1 & 91.5 \\
\hline & Secondary & 1 & 2.1 & 2.1 & 93.6 \\
\hline & Trades & 2 & 4.3 & 4.3 & 97.9 \\
\hline & Undeclared & 1 & 2.1 & 2.1 & 100.0 \\
\hline & Total & 47 & 100.0 & 100.0 & \\
\hline
\end{tabular}

Table 6: Survey participants' level of education

Table 6 shows that $91.5 \%$ of the participants had a postsecondary level of education, $4.3 \%$ had trades certification, $2.1 \%$ had a secondary level of education, while $2.1 \%$ was undeclared. A significant percentage (51.5\% or 24 out of the 47 respondents) had a postgraduate level of education.

Even though this table shows that more than half of the survey respondents have postgraduate level of education, it is important to reiterate that literacy skill - as measured through formal education - does not equate to specific subject knowledge of the issues being discussed. Neither does this formal education make up for the technical knowledge about complex issues such as gender-based violence, girl-child education, or women's Internet access - the three issues on the digital action campaign agenda from 2012 to 2014 .

DiMaggio and Hargittai observe that: 
We know very little about what explains inequality in the know-how or competence needed to find information on-line. While basic access to the medium is increasing, evolution in Web site construction and continual growth in the volume of information flooding the market requires more skills for efficient use of the medium (p. 10).

Education is an important skill that is connected to an ability to use the language of discourse and also understand the subject and context of issues being discussed. Bourdieu (1986) identify cultural capital as an essential element for addressing issues about lack of skill in relation to digital inequality. Dahlgren (2012) has identified online space as an avenue for emerging and fledging intellectuals to develop their skill of speaking truth to power. There is a need to critically assess people's social and cultural capital to determine whether or not individuals have the necessary skill to engage in public sphere deliberations. The case of Nigeriens offers a good example of why this is important, especially for informing changes in government policy about Internet penetration and use in urban and rural communities (Alzouma, 2013). Similarly during the Arab spring movement, women were central to the success of the protests that occurred in Egypt and Tunisia (Faris, 2013). The women who had access to and were literate in the use of social media played a significant role in the protest. In another context, language and the tension around which languages to privilege in global discourse influences how much of the civic engagements in the public sphere can be done at a global level. For example, as we will see in the data being analyzed, the Google Translate tool on WPc is considered insufficient for those who do not speak English to participate fully in the discussions on the website. 
DiMaggio and Hargittai infer that, "Internet competence is related directly to individuals' capacity to use the Internet for the purposes they choose" (p. 11). With regard to inequality in skill, the codes used for assessing the data to capture this third broad form of inequality are: digital literacy, capacity building at an individual level, team based capacity building, capacity building at an institutional level, and language. These codes are used to assess the data from the DACs and interviews.

\section{Digital Literacy}

In terms of digital literacy, this code refers to evidence or indications of pre-existing competence in the use of digital technology and devices. In response to a journal entry about the opportunities that could arise from greater digital literacy and access, PohChing posts the following comment:

When women are digitially educated, they can make some "noises" to appeal and spread news about women empowerment instead of having no easier avenues to create news that are worthy (WWTW $11)$.

Sherna Alexander, who wrote the initial journal entry, responded: 
Thank you for your wonderful comment and your encouragement. Yes the internet is a place where women can make a whole lot of noise for their various causes (WWTW 11).

This exchange was part of a wider discussion about the efficacy of online activism. If the participants are labeling their online work as 'noise', a label that has been tagged onto online activism for decades, what are the chances of their work being taken seriously outside their circle of activity? How can the impacts and results of what they are doing be evaluated as contributions to the wider discussion regarding issues for which they are advocating? Such self-labeling of otherwise serious issues further problematizes and highlights the compounded challenge of lack of full understanding of the complexity of language, and the ways in which such labels can be disempowering.

Loyce in Uganda refers to pre-Internet practices that were used to combat violence against women:

During the training, a 59 year old participant shared an experience of how women in the olden days creatively used local platforms to combat violence against women. She shared that when she was growing up as a young girl, women used to look for a strategic location in the village which was usually an anthill. The woman would stand on top of the anthill and start shouting about the bad behaviors the husband has and she would say "my husband is bad, she beats me when I give meat to the 
children, he is a glutton, and he abuses me all the time..." This was to let every one in the village hear about her husband's bad behaviors which would prompt the villagers to gossip about him, musicians would compose songs about his bad behaviors and he would be ashamed and eventually change his behaviors.

Today, technology has provided us with much more advanced platforms compared to an anthill such as mobile telephones, internet, computers where we can make noise about VAW so that the whole world can hear us. Unfortunately, most women lack the technical know-how and resources to access such tools and fully utilize, engage and participate (WWTW 53).

\section{SEE THE PRINT VERSION FOR ACCESS TO THIS CONTENT}

Image accompanying Iffat Gill's journal entry titled 'ChunriChoupaal - A Meeting Place for Women to Get Access, Learn and Connect' for the 'Women Weave the Web 2014' digital action campaign. 
Carolepng's journal entry is concerned with her experience using digital media in Papua New Guinea to make connections online, and the effects these online connections have on her work in her local community:

Online Empowerment most definitely leads to greater achievements offline and I continue to see success in these, through my community organisation, through my freelance ICT social enterprise, and through the many online social networking discussion groups that have given many a voice to speak on issues that were once taboo, and that were once only discussed behind closed doors (WWTW 8).

Mauri's journal entry identifies computer-programming knowledge as an important element of digital literacy, which she believes is not receiving as much attention as it should in women's circles:

In my feeling and experience, there is a dimension of digital literacy which is not (necessarily) related to "communicating", at least apparently, and has to do with computer programming. Anyone of us (after proper mentoring ;-) ) can discover we all can write programs. This is an important step in developing a rightfully skeptical and practical attitude towards technology. 
We're literally surrounded by computers, most often in the form of hard-to-perceive embedded system (WWTW 31).

Judyannet then commented on Mauri's journal entry above:

Without a doubt, we need to reach out to those who are still familiarizing themselves with computers and lift them up. The other point which is quite striking is the programming bit. If we(women) are to own the webs then we have to dive in completely. We have to learn every bit of it regardless of the technicalities. As such it is with great pleasure that I recognize the efforts of organizations and people who are introducing young girls and women to the exciting world of programming (WWTW 31).

This discussion about the need for women to have a good knowledge of computerprogramming feeds into Bourdieu's observation that skill is an indicator of, and can be transformed into, social and cultural capital. Through the foregoing discussion, we can see that the women themselves recognize that the lack of or inadequate technical skill to engage in the production of cultural artifacts, such as computer applications, can limit what they are able to do with the applications or how they can influence the direction of use or appropriation of such artifacts. 
Interview participants who indicated they had autonomy of use and digital access that is based on adequate technological devices connect these capabilities to affordability of use of the Internet and WPc platform specifically for furthering their goal of participating in a community of people working towards a more just society. The participants have a fairly good level of media literacy that does not necessarily equate to knowledge of the issues being discussed. This affordability of access and autonomy of use further helps the individuals to better understand the issues being discussed as they are able to spend time finding out more about the issues either on the WPc platform or other online platforms to which they belong.

Individual Capacity Building

We can make a distinction between possessing new media technologies like mobile phones - which requires economic capital - and appropriating the technologies - which requires cultural capital. Cultural capital is needed for one to take advantage of the technology that has been acquired through economic capital (Bourdieu, 1986). The individual capacity building code refers to discussions about building community, enhancing social capital and career status, and helping family and friends. Indicators include references to and evidence that a participant uses their skill and knowledge to assist others or gains additional skill and knowledge through their interactions on WPc. The previous journal entries and comments address issues of digital literacy, and how the lack of soft skills in the use of digital devices can further endanger people, as well as expose women to violence. The need of training for employees in government agencies, especially in the developing world, was a recurring 
theme across the three DACs. Tressa kentner refers to this need in her journal entry for the 'Ending Gender-Based Violence 2012' campaign: 'the difference that training for judges, prosecutors and defense attorneys can make in the prosecution of crimes against women" (EGBV 2).

Antego2010's journal entry discusses the prevalence of male-child preference over female children in African societies, but also highlights the blind spots that pit women against women. She draws on her family history to discuss and argue that there are exceptions to the maltreatment women face as a result of men's desire for male children and the blame that is heaped on women when they do not produce a male child, especially in some Nigerian families:

It would be unfair for me not to mention the fact there are African men who treat their children equally and give them the same opportunities for development. My father treated us equally, he always said to us that the difference between men and women was purely physical and that anything else was left to us, we could go as far as we wanted, reach as high up as we could and to never let anyone treat us as anything less than equals, thus the reason for my feminist tendencies (EGBV 17).

Nicole Musimwa's journal entry in the GTTW campaign backs up Antego2010's account of similar experience as recounted in the EGBV campaign. Nicole attests to the patriarchy 
that is still prevalent in the Democratic Republic of Congo, but also suggests that there are exceptions:

I have learned just how fortunate I am being born into my family. I enjoyed equal support with my older brothers when it came to education ... So my friends and I raised the money to help her finish that school year. The following years she worked as a tutor for younger children and was able to earn enough money to stay in school. Today she has her diploma and we are very proud of her (GTTW 16).

TJ's journal entry discusses the influence of her parents on her academic development from an early age through to postsecondary education in Guyana. She points out that although women have equal opportunities to access education, they are still mostly clustered in the social sciences as opposed to the STEM:

They have taken a personal interest in my studies. Whenever they saw a drop in my grades I'd get something akin to what I believe the royal inquisition would be like. I remember one year when I failed math, my father sat with me almost every night for the holidays following that term and worked with me until I was up to par. My mother always ensured that while I studied I'd have food - it would appear and the empty plate would 
disappear without me leaving the table and the assignments I was working on (GTTW 26).

These entries appeared in the 'Ending Gender-Based Violence 2012' and 'Girls Transform The World 2013' campaigns. They are significant because considering that the women making these contributions are located in the global South, their comment that patriarchal underpinnings do not overwhelmingly disadvantage them suggest that they are aware of the discourse of patriarchy and its wide-reaching influence on women's involvement outside of the domestic sphere. By extension, the articulation of individual efforts the WPc members make to enhance their skills, build socio-economic and cultural capital, and educate themselves on the issues are indicators of the women's investment in and commitment to the communal issues they face.

Team Based Capacity Building

The team based capacity building code refers to community and group participants growing as a team based on their interaction on and off WPc. Paola's journal entry discusses the privileging of male over female children and adults in Guatemala, and how this has spilled over into the labour market. She explains that women's work making crafts is not considered part of the formal economy. She details the support her group is receiving from international organizations to give women access to digital technology and assist them in scaling up their work, by connecting them with markets outside of their immediate communities through websites such as YouTube: 
For this reason, in my work with the community library, Fuente de Saburia, we have launched the initiative to promote the comprehensive development of women. Forming in 2007, the "group of women from the library" have facilitated workshops to promote self-esteem, personal development, crafts, cooking, promotion of local products, and many others.

Most of our users are entrepreneurs and have now benefitted from a series of workshops on entrepreneurship. They have since used these skills to promote their abilities and products on the Internet. From this, they can identify a market to sell their products which will in turn lead to a better quality of life. Perhaps we are dreaming too much, but everyday we take a step with these women who are fighting; all they need is a bit of encouragement and some resources to enable them to embark on a path of opportunity (WWTW 44).

Siatta's journal entry refers to the efforts of community organizations to empower female journalists in Liberia and ensure they have the necessary skill to tell the stories in their community, especially after the civil unrest:

In an effort to empower female journalists and build the capacity and self-esteem of women working in the media, the Female 
Journalists Association in collaboration with her partner organized a three- phase professional training which includes computer and internet journalism training. The goal and intent of the initiative is to provide participants with the knowledge on internet blogging, sharpen their skills on professional internet broadcasting, identify and research issues of relevance and concern to create anti-violence campaign using the internet and cell phones. 15 female journalists were trained in the above skills but due to some challenges have not been able to implement what they were trained for (WWTW 41).

Olutosin's journal entry talks about her experience discovering WorldPulse.com and the opportunities that came with the connections she made on the website:

The journey became so sweet and rewarding, enjoying it alone is anemic, therefore, I made a conscious decision to introduce world Pulse to every woman who can read and write in my community. I did not stop at that, I went ahead to solicit computers from my sister, she donated 4 complete computer sets to my organization and we organized free computer training for poor women alone in 2012 (WWTW 55). 
Capacity Building - Institutional

The capacity building - institutional code refers to evidence of institutional support from agencies such as the police, schools, government, community, and religious and corporate institutions. kkibaara's journal entry addresses the issue of soft skills and the inequality women experience, even after the majority of governments and other institutions have attempted to close the digital divide. She discusses the challenge facing women in Kenya, and points out that there is a need for institutions to invest in soft skills so that women can take advantage of the digital technology equipment that is already in place:

It is not strange that many women in the world but in Africa especially give technology very little preference because she has so many other problems to deal with. A woman in the developing county has to grapple with poverty, hunger, lack of proper shelter for her family, drunkard husbands, troublesome teens etc and visiting the internet will be the last thing on her mind. But we have the other kind of woman who is employed, in a good job and she can access internet but you will find that some employers prohibit access of internet during working hours, some even block access to certain websites and this hinders how much a woman can access the internet. Others do have access to internet but they do not have the knowledge of how to get information using the search engines and are afraid to ask for assistance. So you see they have access to 
internet but they do not know that there is so much information in there that they can access and be of much use and help (WWTW $34)$.

In another journal entry for the WWTW campaign, anakarenart makes the point about taking online campaigns offline. This is made possible with institutional support from civil society organizations with which Epic Queen is involved:

I do believe that online empowerment can lead to offline empowerment. We are just doing this with Epic Queen, we create the content in internet but we take action offline with the Epic Queen Chapters in different Places (WWTW 15).

The team based and institutional capacity building discussion raises multiple issues about the exclusion of women's work - as mothers, caregivers, homemakers - from the formal economy of the political state, while simultaneously highlighting the collective efforts of community and civil society organizations to work out capacity building opportunities for women in the digital domain. At the same time, the inadequacies of government institutions in this space are laid bare. For most of those contributing to this debate, institutions such as schools, law enforcement agencies, religious organizations and corporate private companies can do more in this space to ensure digital capacity building opportunities for women. 


\section{Language}

Language competence is an important factor with regard to digital literacy (Emden and Midgley, 2013; Tully, 2013). In the cases of WPc members posting journal entries in other languages, volunteer community members translate these initial postings into English. Sometimes however, when other community members post their comments in English, there is no follow up translation into the language of the initial post. An example is shown in the following exchange from the WWTW campaign. The original post was by begeziani and was made in French:

\section{HARMONY: WOMEN AND INFORMATION}

\section{TECHNOLOGY WITH LACK OF ELECTRICITY}

As far as we are concerned, we lack electricity in the cities of the Bukavu, we are facing difficulties here my dear friends, and we are suffering. We always sleep in the darkness and they are children who cannot sleep in the darkness. But we are used to live with electricity like others are used to stay without light. What can we do to have electricity? We are really already in distress and afflicted by this lack of electricity. The most painful thing for us is the impossibility to charge our cell phones and the other electrical devices we have in the house; we even have devices or equipment that are already not working due to this cut and back of electricity. We do not have enough money so we 
can charge our phones in publiphones all the time. That is why we, women, discuss a lot about the lack of electricity. Thank you for understanding.

Kadidia: Begeziani, I understand the daily difficulties that you encounter in your community due to lack of electricity. This deteriorates your quality of life and also cut the entire community of the world. Do you have the opportunity to sign a petition by all the women in your area and send it to the authorities to let them know the official way, what are your living conditions, real? Doumbia Kadidia

begeziani: Thank you very much for understanding is good commentere of my diary; thank you very much my friends Estheur (WWTW 24).

Arguably, one can use the Google Translate app on the WPc site to convert text back to the language of the initial post, but as I have indicated in previous chapters, this translation tool is not particularly reliable or effective. In the case of the previous exchange, begeziani followed up with a reply in English. In a few other cases however, there was no follow up response from the journal(ist), and this raises the question of whether the person could not understand the comment because it was written in English; and perhaps the possibility that the person was not even aware that she could use the Google Translate tool to convert the 
response to their language of choice. One of the interview respondents who works with a group of women in her community offline in her country also brought up the issue of language competence as a barrier for some of the women who access WPc at a cyber café.

Other accounts of language barrier as a marker of inequality in skill include Gunu_K's journal entry about her work representing the Dalit caste people of India and the challenges she faced trying to do this work online:

The main challenge in finding a voice online was initially to write in English because there were very few online fora in Hindi when I started out. My English was not so good and so I had to get my posts edited before putting them online and that took time (WWTW 47).

Adele Wasolu's entry in the EGBV campaign is one of the examples of journal entries that community members translated into English from French, but Adele did not post comments in response to the comments written in English (EGBV 31). Perhaps it is just a case of her not following up on her journal entry, but it could also be a case of her not engaging with the other community members because she could not use English, and was not aware that she could use the Google Translate app to convert the comments into French. A few other journal entries were written in French, Arabic, and Spanish. 
Interview respondents hold divergent views on the effect of English as the primary language of communication on WPc, in relation to other languages that community members use for posting journal entries and making comments. While some respondents say that the languages do not affect their use of the website, others found language to be a barrier and this somewhat limited what they could do on WPc; there was also indication of an awareness of the Google Translate tool, which appears to be the default means of addressing the language needs of the WPc community:

Emeka: Most conversations are in English and I am okay with that. Some in French, I can read and understand, but wouldn't reply in French.

Chika: [Name of country]-my country- is a francophone country. Fortunately,however, I am English educated and turned out almost naïve in French. It was very easy for me to get along with the language. In my practical life, I've been using English in my university years much more than Arabic.

Uloma: I am a native English speaker, so World Pulse is easy to access. I can read enough French to also contribute to the French writers. Google translate is a nice tool, but I am not sure if it fully conveys full intent of the writing when it is used.

Ifeoma: I am pretty much comfortable in using English language to communicate on the website, though there are many languages available on the site. But, as such I have never faced any problem in 
communicating with the members that has affected our use or modes of communication.

Ogechi: The language don't affect me but do affect many of my [cyber café] colleagues, in that they are somewhat limited in who they can interact with on World Pulse, not being fluent in English. But those that are truly interested in developing a global network do take the time to use the translation tool and also attend the English training classes that we hold at the Center to build their English skills and practice communicating in English. (NR)

Some of the interview participants who are comfortable using English language on WPc consider this ability an asset yet articulate a difficulty in that they do not engage fully with contributions that are made in the French language. Consider also the native English speaker - another interview participant - who, although she finds WPc accessible because she can also use French language, also expresses awareness that the Google Translate app does not fully support language accessibility.

Socio-Cultural Inclusivity

The case of the Arab spring indicates that groups (usually socio-cultural and economic elites) who had access to the Internet used the opportunity to support those who were protesting on the streets of Cairo and in other parts of Egypt (Faris, 2013). Bourdieu (1986) also reminds us of the importance of social and cultural capital in public sphere interactions. 
The story of the three $19^{\text {th }}$ century Brazilian women identified by Gordon (2003) indicates that they used their access to the public domain to encourage and support other less privileged Brazilian women to challenge the public-private dichotomy that was prevalent in Brazil at that time. With regard to inequality in the availability of social support, DiMaggio and Hargittai write that:

Based on these observations about competence, we would expect inequality in competence to deepen inexorably, as skillful users find the Internet rewarding and acquire greater skill and users without know-how are frustrated and turn away. Yet we know that this is not the case: most new users do become more competent (p. 11).

Based on this observation, DiMaggio and Hargittai argue that, "social support of all kinds increases users' motivation to use the technology and the extent to which they develop their own digital competence" (p. 12). Information from the DACs, online web survey and interview data is used to assess DiMaggio and Hargittai's claims about the efficacy of social support in the use of digital technology. The units of analysis used for assessing the data are digital inclusion and support. Table 22a shows the results of survey data that examined the connection between the digital action campaigns and the 'Voices of Our Future' citizen journalism training program as tools of support for WPc community members. Detailed information about this connection is available in Appendix 4. 


\section{Questions 18 and 14: Have you taken the Voices of Our Future training on WorldPulse.com? * Have you participated in any of}

the Digital Action Campaigns on WorldPulse.com?

\begin{tabular}{|c|c|c|c|c|}
\hline \multirow{2}{*}{\multicolumn{2}{|c|}{$\begin{array}{c}\text { Have you taken the Voices of } \\
\text { Our Future training on } \\
\text { WorldPulse.com? }\end{array}$}} & \multicolumn{2}{|c|}{$\begin{array}{c}\text { Have you participated in } \\
\text { any of the Digital Action } \\
\text { Campaigns on } \\
\text { WorldPulse.com? }\end{array}$} & \multirow[b]{2}{*}{ Total } \\
\hline & & Yes & No & \\
\hline \multirow[t]{2}{*}{ Yes } & Count & 12 & 3 & 15 \\
\hline & $\%$ & $80.0 \%$ & $20.0 \%$ & $100.0 \%$ \\
\hline \multirow[t]{2}{*}{ No } & Count & 5 & 9 & 14 \\
\hline & $\%$ & $35.7 \%$ & $64.3 \%$ & $100.0 \%$ \\
\hline \multirow[t]{2}{*}{ Total } & Count & 17 & 12 & 29 \\
\hline & $\%$ & $58.6 \%$ & $41.4 \%$ & $100.0 \%$ \\
\hline
\end{tabular}

Table 22a: Cross-tabulation of 'Voices of Our Future' participation and digital action campaign participation

Table 22a show that whether or not someone has participated in any of the digital action campaigns on WPc is associated with whether or not they have taken the "Voices of Our Future training $\left(\mathrm{X}^{2}(1)=4.171, \mathrm{p}=0.041\right.$; applying Yate's continuity correction $) .80 .0 \%$ of those individuals who have taken the training had participated in the digital action campaigns, compared to $35.7 \%$ of those individuals who had not taken the training. There is a very strong association between the DACs and VoF participation that suggests the communal relationship fostered during DACs potentially encourage participants to apply for and take the VoF training.

The interview data indicated that some interview participants have enough competence to not require any support while interacting on WPc. Other respondents who need assistance 
either know who to ask for support or recall having received such support in the past that has encouraged them to continue participating on WPc and other online platforms. The support received on WPc has a ripple effect both on the website and other Internet sites the respondents use. Amara writes "The assistance made me to accept WP as my own community. A Refuge in time of sorrow. A home where I'm always welcome and a family where I can share my burden" (NR). Ifeoma replies "It really does help me to personally stay connected with the WP when there are people who always care for you and wants to provide every kind of help to make easy our usage or access" (NR).

\section{Digital Inclusion}

Digital inclusion is understood as attempts by WPc community members to be inclusive, including offering to assist with skill acquisition and knowledge about the use of digital devices. Digital inclusion is also connected to indicators of offline support participants receive to use the WPc platform. Miria's journal entry about the strides community women are making in Uganda, especially the culture of the younger ones acquainting the older ones with the Internet, is relevant here:

At least 4 mothers in the past few weeks have with pride narrated that their teenagers have initiated them into some form of social media. This should be scaled up and extended to more mothers/women to enhance social and business communication. 
The young, web compliant generation is slowly but surely taking the lead in transmitting all kinds of information (WWTW 2).

Interview participants also articulated assistance and support specifically on WPc and the effect the assistance has for them. Nkem wrote that "It encouraged me to continue using WP because it was easy" (NR). Emeka responded "Webinars, comments of other people on blog posts and resource sharing such as scholarships, conferences, etc" (NR). Uloma wrote "I will say that as an [volunteer] we were asked our input about the site and we gave out feedback to what is easy to use and what it is not. I feel like they really listened to that feedback when they redesigned the site" (NR).

\section{Support}

There are indications of support and encouragement (spiritual, inspirational, practical, sympathetic, empathic) in the form of prayers written in the comments in the journal entries for the DACs or offers of prayers and other forms of spiritual assistance. WPc has had numerous examples of community members offering support online, some of these in real time, soon after the journal entry has been posted, but mostly days and weeks after the event. By way of example, consider torilynnfox's comment on Gloriabit's journal entry about the plight of girls in Mwenga, Democratic Republic of Congo:

I know it must be emotionally exhausting at times to recall all the troubles women in your community face. But speaking out like that 
can only bring positive change. Your courage and strength will be so influential and inspirational to other girls in your community and all over the world. Keep up the good work. We are all listening! (GTTW 22).

What is brought out by some of these exchanges is that inequality and injustice are not specific to any one community, although certain regions of the world experience inequality and injustice more than is the case in other regions. Institutional cultures and practices are a major factor in the struggle against injustice and inequality. Through the submissions in their journals, WPc community members were able to identify how inequality and injustice have become systemic and perhaps more difficult to fight because of the institutions that are used - directly or indirectly - to uphold the practices and power relations that produce inequality and injustice.

Mukut's journal entry addresses the issue of the systemic institutionalization of abuse. She talks about how religious (Hindu) texts and practices, as well as the entertainment (Bollywood) industry, help perpetuate the cycle of domestic and street sexual violence in Indian society. With regard to religious texts, she writes that:

The Maitrayani Samhita (Yajurveda: one of the four canonical texts of Hinduism), repeatedly says that a woman's body is not her own, so she cannot prevent herself from being molested. The current 
ministers (including some of the women leaders) repeatedly blamed women for 'inviting' the offenders (EGBV 23).

The example she referred to was 'eve-teasing', a form of street sexual harassment that she acknowledges is prevalent in India, but also common all over the world. She also writes about the way media texts encourage and naturalize symbolic and physical gender violence:

'Bollywood', our very own Hindi cinema industry, churns out movies year after year for the public. And scores watch them, sing to their tunes, and emulate them, trying to be the next 'hero' or the 'villain' on the streets. Some depictions in Indian cinema show mild teasing as part of flirtatious beginnings of a courtship. 'Pinching' of the stomach or trying to run away with the 'dupatta'(the long scarf that a woman carries or wear it with a suit), are common ways in which a 'hero' accosts the 'heroine'. The 'heroine' is shown to be submissive and coy, giving in to the 'hero's advances by the end of the song. Young men tend to emulate the same thinking that they too can get away with same kind of behaviour with similar results. The Police/ constable's outlook towards the issue is equally deplorable. If at all a woman, gathers the courage to complain at the police station, the officer makes it a point to make matters worse for her. His smirk and the 
look on his face is enough to express that he does not believe a word of what you said, or better still, does not care (EGBV 23).

The journal entry by chargerrose 7 develops the debate about the assumption that issues regarding female child education are specific to so-called 'less developed' countries. She writes that:

This assignment has been one that has led me to a deep level of introspect. While I see so many barriers to receiving an education in other countries, here in America we should have more opportunities, less barriers .... but do we really? The challenges I faced in my community growing up were based around my religion. The religion I belong to encourages girls to focus on married life from an early age. Once the men get back from their missions we are encouraged to marry. This leads to most girls being married by 20 or 21 . I was married at 22 . Many, once married, become housewives and mothers right away. Working outside the home, or continuing their education is not looked upon with favor. We are to dedicate ourselves to our husbands. Many girls that were in school, quit and never go back. While I love my God, and I love the religion in my heart, and I see that there are some women that enjoy staying home and never going further in education, this mindset hinders some girls and women from 
becoming perhaps the Doctors who would find cures so diseases, who knows ... perhaps one of them were destined to become the first Female President but will never see this become a reality. Some may say that this is not a true barrier. Many may say that this is just a belief set that women do to themselves. "they could choose to go on if they wanted to" I've heard. But many cannot. Cultural barriers run deep (GTTW 23).

In her post, chargerrose 7 identifies religious and cultural attitudes as two factors that limit girls' access to higher education in America. Her post feeds into yet another ongoing public sphere debate that communities in developed countries need to be as vigilant about their internal societal problems and inequities. This need for vigilance is highlighted in the UNESCO 2015 report of the 15 years of the Millennium Development Goals ('Education for All 2000-2015', 2015).

WPc community member Maya Norton further develops the discussion about religion as a possible barrier to access to education in America. She asks chargerrose 7 if she is of the Mormon faith, perhaps suggesting that the practices described by chargerrose7 are prevalent among that religious sect:

ChargerRose, your first journal entry hurt my heart. Have you found people, especially women, to connect to since your first writing? Depression is never ever the individual's fault. You are 
brave to write about it and ask for support and help. It sounds like you are Mormon since you mentioned missions, right?

The bind you have described between children and education as important parts of life are one that so many women face. You are not alone. Thought you might be interested in Bitani's piece, the first submission of the week, that touches upon some of the issues you wrote about. I'll send her your link as well http://worldpulse.com/node/69153 (GTTW 23).

Diane Ezeji, another WPc community member who is American, also identifies religious beliefs as a barrier to accessing education, but expands this by identifying rural and isolated or closed communities as additional factors:

I also grew up in a conservative religious environment. Women were told that their goal was to be a wife and mother. I felt strong discouragement in expressing an opinion or having an interest outside of those two goals. Women were taught to be silent and that if you wanted to be heard it was because you wanted attention (a negative need in their minds). I feel that growing up in that environment still handicaps me today. When I go to a job interview, for example, I feel awkward telling them about my abilities; that was seen as bragging. Men were supposed to make the decisions for the family, but what if that man made poor decisions? The 
whole family suffered in silence. I got away and got my education, but still struggle to find my voice. I think the majority of the US population aren't aware of what is still taking place in rural and closed communities like the ones we know. Thanks for your bravery (GTTW 23).

There is a resounding uniformity across all the journal entries, comments, survey and interview responses assessed for the socio-cultural inclusivity factor that suggests skill is a major marker of digital inequality, regardless of location or social status. WPc participants located in Asia, Africa, and North America shared information about the diverse ways they experience skill in relation to access to education, social support from family members, friends or third party organizations, and development of subject knowledge. These are identified as stumbling blocks on the road to participation in public engagement. What is interesting to note, and highly positive, is that despite these challenges, the participants do find their way through multiple channels to access the site of discourse and participate in the conversations about the issues such as girl-child education and gender-based violence. Another common feature is the identification of the media's role in perpetuating systemic inequality and injustice, institutionalization of abuse, and naturalization of symbolic and physical gender violence. This appears to be a major concern for many participants located in Asian and African countries although two participants in the United States also specifically identify religious and cultural attitudes - including specific link to rural, isolated, and closed communities - as factors that hinder girl-child access to education. 
Variable Digital Affordances

As we have seen from previous chapters, skilled civic activists can make use of digital technology - especially social media - to challenge traditional media's gatekeeping and agenda-setting functions when engaging in public sphere activity. The level of use of these technologies differs depending on a person' location: for instance, urban dwellers in Niger have better variation of use compared to rural dwellers in the same country (Alzouma, 2013). Likewise people located in developed countries are more likely to have connectivity for longer periods of time, although this is not always the case for all developed countries. For instance the experience of a WPc community member who moved to the Appalachian Islands in the United States indicates that Internet penetration in that part of America still lags behind other parts of the country.

Pre-existing scholarship indicates that the use of technology as a means of engaging in civic protests is very much tied to specificities of time and place. By way of example, both traditional and digital media were used interchangeably and in various degrees during the anti-Emergency protests in Pakistan (Bolognani, 2010). These protests were articulated along class, economic and educational lines; these are markers of the diversity that undergirds the variation of use experiences of those who engaged in the protests. The use of social media by social activists for civic engagement and social organizations for fulfilling their organizational objectives varies in different countries. In Bulgaria, social activists combined traditional and new media circumstantially during political protests (Bakardjieva, 2011), while social organizations in Australia specifically engage celebrities purposely in 
using social media for meeting their organization's needs (Prince, 2004). There is evidence of the practice of purposeful use of digital media on WPc: the platform combines traditional and new media in their digital action campaigns and the work they do with the women they train in the 'Voices of Our Future' citizen journalism program.

In terms of variation in use, DiMaggio and Hargittai pose the question:

How do income, education, and other factors influence the purposes for which one uses the Internet? We place high priority on examining determinants of different kinds of use, especially distinguishing among uses that increase economic productivity...or political or social capital (p. 12).

They argue that, "education will be a strong predictor of the use of the Internet for the enhancement of human capital, the development of social capital, and political participation" (p. 13). The data collected through the online web survey were used to assess DiMaggio and Hargittai's claim about variation in use of digital technology. WPc community members' experiences were assessed using the codes: digital affordances, digital empowerment - individual or group, and digital empowerment - institutional. Although there are indicators that the majority of the participants are educated beyond secondary level, there is no specific evidence that demonstrates that a specific level of education influences or determines the level of interaction participants have on the WPc platform. However, there is an indication that those who have postgraduate qualification engage consistently more than those who do not. Tables $23 \mathrm{a}, 24 \mathrm{a}$, and 26 show the data from the 
survey that further explain the relationship between levels of income and education, digital action campaign participation and 'Voices of Our Future' citizen journalism training of WPc members. Detailed information from these tables is available in Appendix 5-8.

T-test for questions 7 and 14

\begin{tabular}{|l|l|r|r|r|r|}
\hline & $\begin{array}{l}\text { Have you } \\
\text { participated in any } \\
\text { of the Digital } \\
\text { Action Campaigns } \\
\text { on } \\
\text { WorldPulse.com? }\end{array}$ & $\mathrm{N}$ & Mean & $\begin{array}{r}\text { Std. } \\
\text { Deviation }\end{array}$ & $\begin{array}{c}\text { Std. Error } \\
\text { Mean }\end{array}$ \\
\hline $\begin{array}{l}\text { How much do you } \\
\text { earn per year? }\end{array}$ & Yes & 13 & 21207.69 & $\begin{array}{r}20044.51 \\
1\end{array}$ & 5559.347 \\
& No & 10 & 35856.00 & 56423.89 & 17842.801 \\
\hline
\end{tabular}

Table 23a: Level of income and digital action campaign participation

Tables 23a show that the 13 survey respondents who indicated they had participated in the Digital Action Campaigns reported a mean income of US\$21,207.69.

This mean income is taken from across all survey respondents from different regions of the world, and potentially suggests that these participants engaging in civic duties on WPc are also otherwise gainfully employed and have other work commitments offline that are not necessarily connected to civic activities. 
T-test for questions 7 and 18

\begin{tabular}{|l|l|r|r|r|r|}
\hline & $\begin{array}{l}\text { Have you taken the } \\
\text { Voices of Our } \\
\text { Future training on } \\
\text { WorldPulse.com? }\end{array}$ & $\mathrm{N}$ & Mean & \multicolumn{1}{c|}{$\begin{array}{c}\text { Std. } \\
\text { Deviation }\end{array}$} & $\begin{array}{c}\text { Std. Error } \\
\text { Mean }\end{array}$ \\
\hline How much do you & Yes & 15 & 26220.00 & 46237.681 & 11938.518 \\
earn per year? & No & 15 & 24157.33 & 22579.976 & 5830.125 \\
\hline
\end{tabular}

Table 24a: Level of income and 'Voices of Our Future' participation

Table 24a indicate that there is a link between survey respondents' level of income and their participation in the DACs and VOF. More than half of income earners participated in the DACs while exactly half of income earners participated in the VOF. These are people who are otherwise engaged in the workforce and gainfully employed yet also making time to participate in these activities that are voluntary and require a significant amount of time commitment.

Questions 5 and 18: What is your highest level of education? * Have you taken the Voices of Our Future training on WorldPulse.com?

\begin{tabular}{|c|r|r|r|}
\hline \multirow{2}{*}{$\begin{array}{l}\text { What is your highest level of } \\
\text { education? }\end{array}$} & \multicolumn{2}{|c|}{$\begin{array}{c}\text { Have you taken the Voices } \\
\text { of Our Future training on } \\
\text { WorldPulse.com? }\end{array}$} & \multirow{2}{*}{ Total } \\
\cline { 2 - 4 } & Yes & No & Tor \\
\hline Bachelors & 9 & 5 & 14 \\
Postgrad & 13 & 8 & 21 \\
Other & 0 & 3 & 3 \\
Total & 22 & 16 & 38 \\
\hline
\end{tabular}

Table 26: Cross-tabulation of survey respondents' level of education and participation in the 'Voices of Our Future' training.

Table 26 indicates that nine of the 22 survey respondents who took the 'Voices of Our Future' training have Bachelor's degrees while 13 have Postgraduate degrees. 
Whether or not someone has participated in the digital action campaigns is independent of their level of education. However 10 of the survey respondents who have participated in the DACs have postgraduate educational qualifications, while 13 of the survey respondents who have participated in the VOF have postgraduate degrees. Almost all the survey respondents have postsecondary qualification, an indicator that they do have basic literacy skills although as we have noted previously, literacy can not be equated to subject knowledge skill.

Digital Affordances

The digital affordances code refers to discussions of access to the Internet and digital technologies, as well as to how digital devices enable WPc community members to engage with others online or offline. Lea's journal entry reported on an interview she conducted with a student in Afghanistan. She discusses the challenges women face in Afghanistan and some of the barriers to education due to discrimination based on gender and cultural expectations:

While in the past women had no freedom, some changes have occurred: More women are able to go to school and some are given an opportunity to study abroad. There are also women who have become active in women's rights groups and have founded cafes such as the Sahar Gul café where women can meet, discuss topics relating to women's rights and can educate 
themselves through technology and literature. Now women have the ability to openly protest for their rights and we have now seen women taking to the streets to protest and to demand more equal rights. Hopefully, this trend will continue and will spread as the Afghan society becomes more aware of their impact (GTTW 37).

Melva Sangri's journal entry recounts her mother's experience of using Google Search to help her father regain consciousness after he fainted in the garage of their home. Melva's mother credits her grandchildren for helping her and her husband learn the basics of connecting online and using Google Search to seek information:

I ran to the computer (thank God it was attached because he had spent the afternoon just trying to learn more and apply learning with my grandchildren) and I managed to get to a search engine, and as my husband is diabetic, I looked: "fainting diabetes," those two words actually were the only ones I could think at that moment of desperation. I opened the link and read: "It is important to recognize the symptoms of hypoglycemia and treat immediately appear quickly and can be dangerous if the level of blood sugar is very low, leading to fainting or seizures." I ran to the kitchen took a drink with lots of sugar, I did drink and was slowly regaining consciousness (WWTW 1). 
'Women on the rising's' journal entry discusses how she balances her need of digital literacy with the need for safety. She discusses her experience using the Internet since 2003 in her home country Nigeria and how she navigates spending time at the cybercafé, which is often populated by male patrons. She would later go on to learn computer programming during her national youth service:

When I say I love the web I do mean it. The internet has hooked me up to interesting people, people who help have a focus, discover my hobby and using I'm using the internet to help many others in different ways. From the inspiration I got, I came to realize that as much as every business needs a physical or geographical office space so should they also have an office space or unique face of the business online so I started a business of designing websites and blogs like ukwuomanomso.blogspot.com (WWTW 5).

AlejandraL argues that the Internet is essential for development:

Internet opens the door to new knowledge, broader and much more real that we can come to teach in our years school: the virtual education offer is very diverse and there are opportunities for all ages and economic conditions, and there are even free 
courses or apply scholarships. From my home I could "take" away and get specializations !!!! Internet allows us to generate links with women from other parts of the world and learn about their experiences, customs and societies in which they are embedded, exchange ideas and / or projects, meet other "realities" sometimes very distant (WWTW 13).

Digital Empowerment - Individual or Group

The digital empowerment - individual or group code is about the ability of WPc community members to complete certain tasks due to their knowledge of digital tools or access to digital technology assistance through other individuals or groups. 'Women on the rising"s journal entry discusses how she balances her need of digital literacy with the need for safety. She discusses her experience using the Internet since 2003 in her home country Nigeria:

As for learning something, in $2008-2009$ during my National Youth Service Corp (NYSC) in Jos, Plateau state in Nigeria, I found an Information Technology (IT) Institute offering cheaper tutorials and have authorized Test Centre (Pearson) for IT certifications. I located the IT institute in Jos, enrolled for an Oracle Database Administration training and in that same year I emerged as the only lady in that institute that wrote the 
international exams (SQL, OCA and OCP) making a 100 per cent result in OCP and got certified in Oracle 10g Database Administration (WWTW 5).

Carolepng's journal entry details her experience using digital media in Papua New Guinea to make connections online and the effects these online connections have on her work in her local community:

Online Empowerment most definitely leads to greater achievements offline and I continue to see success in these, through my community organisation, through my freelance ICT social enterprise, and through the many online social networking discussion groups that have given many a voice to speak on issues that were once taboo, and that were once only discussed behind closed doors (WWTW 8).

Mauri comments on her own journal entry about the challenges of navigating online web spaces in the quest for digital literacy and having a good working knowledge of technology:

Sure, "programming" is "doing", on the net, in a bit different manner than using things already built. It's very much about "agency", something not expected by girls and women, yet a 
step I feel useful to become full-fledged digital citizens

(WWTW 31).

Adife Sudkivu's journal entry refers to her experience using the Maman Shujaa Centre in the Democratic Republic of Congo to access the Internet, and how she has gained knowledge of global current affairs through her exposure to communities outside of the Congo:

A working knowledge of technology is of capital importance for us women, to have the chance to express ourselves and to make our own voices heard as well as those of our fellow women who don't have this chance. We thank our Maman Shujaa Center for this great opportunity offered to young women and girls from different organizations and their members (WWTW 42).

Digital Empowerment - Institutional

The digital empowerment - institutional code refers to the ability of WPc community members to complete certain tasks due to assistance facilitated by institutions such as schools, public libraries, and government agencies. There was not much referenced in this area. This could be due to the fact that WPc community members are not aware of such availability, or perhaps because these services are just not available in their communities. 
Overall it appears digital affordances are closely linked to digital empowerment both at an individual and group levels. The participants' time affordability - which is a socio-cultural capital - influences their use of technology for civic duties. Participants in many locations recount purposeful use of digital media for personal and communal needs both online and offline with an indication that level of education is not a significant factor in the deployment of these technologies although other demographic factors such as age influence the level of use.

\section{Conclusion}

The evidence derived from the DACs and interviews indicates that WPc community members' experiences of the use of digital devices for accessing the platform and engaging in civic and public sphere activity vary, largely depending on demographic factors. Their experiences mirror that of other digital device users who - depending on their age, location or economic status - may not be able to take advantage of digital technology for other activities, including curriculum development or use in classrooms. By extension the technology of the Internet inherently features inequality in both its form and dispatch. Economic, socio-cultural, and political capital influences or determines people's ability to use technological devices independently. There is evidence of a correlation between people's autonomy of use of digital technology and what they are able to use the technology for, especially in relation to employing the technology for civic engagement on WPc. 
A practical working technical knowledge and wide cultural literacy, particularly with regard to the issues at hand, are important factors in determining online public sphere engagement. The data across the digital action campaigns and the interviews show that soft skills such as language and subject knowledge have an effect on the kinds of engagement and experiences that community members have on the WPc platform. Through an assessment of levels of digital inclusion and both individual and communal support, it is apparent that people's experience of civic engagement on WPc is enhanced when they perceive themselves as part of a community, and also when their fellow community members support them, both online and offline. Social support offline is especially significant for WPc community members, because they have come to see their membership of the platform as a form of social capital from which they can draw upon when they need assistance in their local communities. Variations in use differ, depending on demographic factors and organizational needs. While civic activists often tend to use digital media circumstantially during political protests, organizations often use digital media purposefully and strategically across multiple campaigns.

This chapter has focused on the five broad forms of digital inequality identified by DiMaggio and Hargittai in order to contextualize and evaluate the culture of WPc with regard to the extent it meets the public sphere requirements and criteria identified in previous chapters. What was interesting about the material used to conduct this evaluation is that access and agenda setting were both identified as significant issues by WPc members. What is also worth noting is the rather difficult and complex relation between the general 
imperative that the public sphere should function as a space for critique, and the culture on WPc, which tended to place a much stronger emphasis on discourses of empathy, support and tolerance.

In the following chapter, I will introduce a civic engagement typology, which will be used to assess the data primarily collected from the digital action campaigns. The material is derived from content analysis of the digital action campaigns, online web survey, email interviews and researcher's journal reflections. 
Chapter Eight: Civic Engagement Typology and the Digital Action Campaigns on

WorldPulse.com

Introduction

In this chapter I will introduce a civic engagement typology, which will be used to analyze the data primarily collected from the digital action campaigns. The material in this chapter is based on my evaluation and organization of the data produced in the content analysis of the digital action campaigns, online web survey, email interviews and researcher's journal reflections. In coding the data retrieved from the three digital action campaigns, I found that there were distinct themes in the contents of the journal entries and comments submitted for the campaigns. Consequently, the ensuing analysis was organized around these themes. These emerging themes are in addition to the assessment of Habermas' public sphere prescriptions, and DiMaggio and Hargittai's five broad forms of inequality conducted in the preceding chapters. The desire on the part of different groups of citizens to engage with others regarding issues of public concern was evident across the three campaigns. Indeed, it can be argued that the act of taking up membership in the WorldPulse.com community in itself constitutes a form of civic engagement. Beyond the initial membership, community members can interact with each other through some of the channels that the WPc management has set up, including leadership and volunteer groups.

A major marker of the level of civic engagement among community members is the voluntary contributions they make in their journal entries during the digital action 
campaigns. These contributions are based on activities that may have already occurred locally in the person's community, or issues or activities of which they may simply be aware more globally. Documenting such activities is indicative of some of the work people do, based on which they are referred to as citizen journalists. However, in the context of this research project, I find the label 'citizen journalist' to be reductionist, considering the magnitude of complex activities that constitute these people's activism. I will use the term 'embedded e-activists' to describe the groups of people who conduct their activism using intersecting and overlapping online and offline tools for civic engagement.

Volunteerism is integral to the work of embedded e-activists. These kinds of activists go beyond just clicking a website link, liking a Facebook page, retweeting a Twitter post or forwarding of calls for action on advocacy websites. These e-activists are embedded, both in multiple global online communities as well as in local offline communities. Embedded eactivists place a high value on their civic engagement activities. These social activists engage actively offline, endangering their lives by working at the intersection of online and offline actions on the streets leading rallies, vigils, and campaigns that they often give follow up updates on through interactive media reports and reviews. The codes that were used to identify levels and forms of engagements of embedded e-activism were: demographics, issues, measures, actions, outcome, expectations, recommendations, solutions, repercussions, volunteerism, authentication, value and collective voice.

\section{Demographics}


Accurate and reliable demographic information of virtual forum members is often difficult to obtain, perhaps because of the fluidity of identity and the temporality that accompanies online personas. It is nonetheless expedient to know even the most basic information about the groups of people participating in a forum, especially so that one can understand the spread of the diversity of opinions, and the relationships between the opinions expressed and those expressing them. Through data collected using the online web survey, I was able to get some demographic information about WPc community members. I then conducted a cross-tabulation of some of the data to explore the relationships between the members and some of the identifying features that will be used to organize, evaluate and analyze deliberations and discussions in the forums. Table 30a show the relationship between age and digital action campaign participation. Detailed information about the survey data specifically for the interconnections in demographics is available in Appendix 9-13. 
Questions 2 and 14: What is your age bracket? * Have you participated in any of the Digital Action Campaigns on WorldPulse.com?

\begin{tabular}{|c|c|c|c|}
\hline \multirow{2}{*}{$\begin{array}{l}\text { What is your age } \\
\text { bracket? }\end{array}$} & \multicolumn{2}{|c|}{$\begin{array}{c}\text { Have you participated in any of the } \\
\text { Digital Action Campaigns on } \\
\text { WorldPulse.com? }\end{array}$} & \multirow[b]{2}{*}{ Total } \\
\hline & Yes & No & \\
\hline $18-30$ & 5 & 5 & 10 \\
\hline $31-40$ & 3 & 4 & 7 \\
\hline $41+$ & 9 & 3 & 12 \\
\hline Total & 17 & 12 & 29 \\
\hline
\end{tabular}

Table 30a: Cross-tabulation of survey respondents' age and digital action campaign participation.

Table 30a indicate that those 41 years old and above are more likely to participate in the digital action campaigns than any of the other age groups.

The hours per week spent on the website is independent of the age of the individual.

Likewise, whether or not someone is a member of any subcommittee on WPc is independent of their age. Of those aged 18-30 six of them spend 1-2 hours per week on the website; in the 31-40 age group, five out of 10 spend 3+ hours on WPc while in the 41 and above age group 8 out of 18 survey respondents spend up to 1 hour per week on WPc. Regarding employment, whether or not someone is in paid employment is independent of 
their age group. The majority of survey respondents across all the age groups are in paid employment (41 out of 46 survey respondents). Whether or not someone has participated in the DAC is independent of their marital status. Presumably this result could change if we assume that everyone who skipped the question about marital status in relation to age did not participate in the digital action campaigns. Overall there are more single people than any of the married or other groups participating in the digital action campaigns. This is perhaps indicative of the fact that the former group has more time affordabilities than the latter group.

Issues: The Comment is the News

The issues code captured discussion of political, social, economic and cultural issues by looking at which of the issues are on the agenda, and the additional issues introduced or referred to by the participants. The issues identified and discussed (or not discussed) during the digital action campaigns are an extension of (but not necessarily limited to) the issues identified by the WPc management. In examining the issues that were placed on the agenda by WPc, along with the additional issues community members raised, there is evidence to support the contention that the comment more or less 'becomes' the news; in other words commentary engages with, argues about and develops the issue, thereby creating additional perspectives and points of contention. However, there is also a sense in which the fact that an issue attracts commentary makes it 'news'; if enough people are interested in or even passionate about something, to the extent that they will engage in or produce public sphere action, then that itself constitutes or brings about 'an event' that both develops and surpasses 
the original topic. Volkmer (2008) argues that technology has facilitated opportunities for the creation of "new discursive event spheres" that are not restricted by the rules of the 24hour news cycle. Her position reflects my observation of the culture on WPc in relation to citizen journalism, and how this culture is growing rapidly in many of the forums. It can be argued that the spreading of this culture of increased online activism facilitates an increase in citizen engagement in and with civic activities.

Moreover, when one examines the issue on the agenda as set out by WP management in comparison to the extended issues that the community members identify, there is an indication of the issues that were originally excluded, but which are clearly significant for the community members. By way of example, in one particular journal entry all the connected codes of 'Actions', 'Issues', 'Expectations', 'Measures', 'Outcomes' and 'Solutions' show up in the narrative that the WPc community member Kotravai supplied; the notion that women are implicated or involved in gender-based violence was raised in the discussion. In her journal Kotravai writes about the case of a mother who was brave enough to report to the police that the father of her daughter's friend raped her 13-year-old daughter. The female police officer Kotravai reported the issue to asked for a bribe and questioned the claim of rape:

I went to ammapettai police station with my daughter. I briefed them everything. S.I. Ramana said give Rs.10,000 we will file case. I said "where do I go for such a big amount" immediately inspector Jayam said ‘hey, wouldn’t you listen to what Ramana 
says, you sent your daughter to flirt around with that man for a week, let her enjoy and now you are shedding crocodile tears is it?” My heart broke, “pl don’t speak like this madam” I said. Then in front of my eyes itself, they asked my girl, where all did he touch you, how did he touch you etc in a very vulgar manner. I pleaded "pl don't treat her like this, she is little girl". They said it is just an enquiry process. Then she said "hey ramana, take this Saturn to the opposite Dr. Sumathi and check if she is truly raped". I said she did not volunteer, but she is raped. She said "both are same ... don't teach me law" (EGBV 5).

Even when the doctor confirmed that there was evidence of forceful penetration and the police agreed to file a case, the girl had to go back to the hospital for additional tests. Another obstacle was the bribe demanded by hospital officials before they would attend to the girl and her mother. This issue becomes more complex as the wife of the alleged perpetrator allegedly paid a large sum in bribe to see the case dismissed:

Then one day Ezhumalai's wife mallika came home and said "I thought I will pay some good money to a widowed mother and compensate, but you went to police, that's why I gave them 30,000 and finished the case. You can do nothing". And threw the report on my face. Here money plays a major role, poor are left without any help" (EGBV 5). 
In the GTTW campaign, education was the primary item on the agenda. However, community members discussed numerous other issues (that were considered to be more important or of equal weight) in their journals. Abby Kakiyes' entry reads:

The common man is plagued by poverty; he has little or no cravings for super wealth as long as the basics are in place being food, drink, power, good road, healthcare and most especially EDUCATION for their children as they were themselves not privileged (GTTW 1).

She extended the discussion to take in issues of terrorism, security and government policies:

The young beautiful girls of Riyom rural community in Plateau State Nigeria have not only witnessed the horrors of genocide but have not seen the walls of a classroom in over one year of being displaced. For these girls, going to school had always been a challenge due to financial reasons especially when there is no access to free quality education or scholarship to support promising students from challenged homes. In the face of the on-going boko haram extremist crisis in Northern Nigeria where women and children are not spared; situations have worsened as their parents 
who have been peasant farmers of carrot, cabbage, potatoes, and other vegetables were murdered, raped and massacred by the Islamic extremists who are politically operating in guise to wipe out defenseless Christians (GTTW 1).

\section{SEE THE PRINT VERSION FOR ACCESS TO THIS CONTENT}

Image provided by WPc community member Sutanuka Banerjee to accompany her post titled Countering Gender Bias: 'Boys have trucks - Girls have dolls”, '

This practice of extending the topic of discussion, which manifests as a challenge of the gate-keeping and agenda-setting function of media producers, is similar to the observation I made after an assessment of other publishing platforms such as cnn.com, cbc.ca, and stuff.co.nz (Giwa, 2015). Through channels such as Twitter, Facebook, Instagram, other social media sites and the commenting sections of news and entertainment platforms, we get more information and insight into people's thinking patterns, and what they are thinking 
about. Sometimes these people give information about why they think the way they do and why they choose to engage with specific topics and causes.

Measures

The measures code refers to instruments that exist or are being developed to aid in addressing the issues and solving the problems. Implicit in these measures are the assumptions that the proposed ideas are within the reach of the participants and that such measures can possibly offer the solutions needed. The measures code was further broken down into the following sub-codes: individuals' measures, institutional measures, and measurable actions.

Individuals' Measures

Mitukhurana's journal entry about female feticide in India is relevant here. She referenced the film It's A Girl in sharing information about the steps a female pediatrician took to save her daughters from the prejudice and persecution meted to them due to their gender and birth into an Indian family:

Mitu filed a complaint under the Pre-Conception and Pre-Natal Diagnostic Techniques Act (PC\&PNDT Act) against her husband as well as the doctors and the hospital that preformed the illegal sex-determination test (EGBV 3). 
Kotravai's journal entry describes the measures a mother took to report to the police that the father of her daughter's friend raped her 13-year-old daughter:

Mayan, Human Rights Protection center member volunteered Legal help to Kavitha. He said that "Forensic report is not supposed to be let out, but the opposite party had taken it, this shows that how bribary has played a major role in this case. They refused to file a case, then Kavitha threatened to self immolate her. So they filed the case. They have influenced the concerned with money and changed the report. We have appealed for further investigation." While we contacted Inspector Jayam she said that “I have acted as per law and ensured punishment for the convict" (EGBV 5).

Another of Kotravai's journal entries details the case of a woman who was bathed with acid after being sexually harassed. Kotravai then gave details of the measures a community organization took to assist the victim in seeking justice:

Reading the above news in Hindu, MASES immediately got into action by publishing the news on its blog \& via social Media and took it to the notice of more People. Reading about the injustice done to Sonali, I was highly annoyed and wanted to do what 
best can MASES do to appeal to People for help. We posted about the issue on MASES blog on 12th July and wrote letters to Commissioner of Police Delhi, Chief Minister Delhi, AIDWA, National Commission for Women, Ministry of Women and Child Development. We also approached few Human Rights Organization and NGOs seeking letter of Support and to urge the Ministries \& authorities to intervene (EGBV 54).

Institutional Measures

Some of the journal entries and comments report on the measures that institutions are taking to address the issues the participants identified. Mukut's journal entry refers to the legal measures that are in place to address street sexual harassment in India:

What the Law says under IPC: I find it of utmost importance to highlight here what the law says regarding street sexual harassment. As mentioned before the Law does not recognise the term 'eve teasing'. It is a mindset construed to insult women and their dignity. Section 292 of the IPC clearly spells out that showing pornographic or obscene pictures, books, draws a fine of Rs. 2000 with 2 years of rigorous imprisonment for first offenders. In repeated offence, he is slapped with a fine of Rs. 5000 and 5 years of imprisonment. Under section 509 of the 
IPC, obscene gestures, indecent body language and acidic comments directed at any woman or girl carries a penalty of rigorous imprisonment for 1 year or a fine or both. But it is a bailable offence. Note-worthy action taken in some cities: With the increasing incidences of harassment in Kolkata and other cities, and ineffective law, the women constables, have decided to take up the matter in their hands, and they have started functioning as undercover agents by acting as decoys in the areas most afflicted. Their stint has been successful so far with more than 60 offenders held in less than two months (EGBV 23).

WorldPulse.com management's summary of the 'Girls Transform The World 2013' campaign reports on the measures taken by the organization to present the community members' deliberation at a global forum:

These submissions were then taken into consideration by the 21 youth delegates to the G(irls)20 Summit before delivering their recommendations to world leaders at the G20 summit in Moscow (GTTW).

Iffat Gill writes about the measures that government and communication businesses are taking to ensure the safety of mobile phone users in Pakistan: 
The strong need to adapt to swift changes in the technological and the personal landscape of its young population, calls for the importance to educate the young population about privacy and safety issues attached with the risks of their current behaviour. The situation has been under scrutiny since the decision of PTA to ban all unregistered sim cards and connections. It is illegal to be in possession of a SIM card if it is not registered. This, to some extent has assisted in tracking people who 'prey' on unsuspecting women, making it less difficult for them to hide their identity. No one educated Nazira about the pros and cons of interacting with strangers via the new technologies. Therefore, a country wide privacy campaign and outreach program is needed to minimize the threats associated with it. An informed decision always supersedes an uninformed one (WWTW 21).

Measurable Actions

The measurable actions sub-code examined the changes in policy and mode of governance in the micro communities and larger macro communities of WPc members. During the digital action campaigns, WPc community members offered suggestions about how to solve the problems of endemic and institutionalized violence against women, girls' limited access to education, and inequalities of digital access. Tressa kentner writes that education for both males and females (as a tool of empowerment for ending violence against women 
and girls) and as economic empowerment for women and girls were both central to bringing about greater gender equality:

My vision for ending violence against women is that every girl and every boy would receive the education that they need to lead healthy, fulfilled lives and that this education would lead to an understanding of the value of every human being regardless of gender, ethnicity or religious affiliation (EGBV 2).

Walaa Salah's journal entry refers to the disparities between male and female students at universities in Sudan, especially after the introduction of Sharia law. She suggests measures that could be taken to address these disparities so as to enable females fulfill their dreams:

For me education is not only about classes or lessons, education should be meant to empower women and encourage them to fully participate in society to make women envision the future with optimism and belief in better world. Reforming discriminatory laws, considering to the quality of education, carefully drafting messages which teachers are delivering, and before all granting freedom! This what paves the way for girls to harvest the fruits of their education (GTTW 12). 
WPc community members made various suggestions about how to alleviate some of the problems identified by other WPc community members. Lydiagcallano commented on Siatta's journal entry about challenges of digital access in Liberia:

I would like to believe that Liberia will one day have an improved ICT scenario. As prices of computers and communication systems go down, more people will be able to afford personal computers and cellular phones with Internet connections. Also, your Internet connections will likewise pick up in speed while its cost will dip (WWTW 41).

In some cases, for instance in Amina_3's journal entry for the 'Girls Transform The World 2013' campaign, community members focused on the plight of women's internal struggle to challenge family and government institutions whose expectations are firmly rooted in patriarchal doctrines. In discussing her struggle to access education in India, Amina_3 described having to convince her parents to change her school more than once, including moving to a different part of the country to ensure she attended a good school:

The first and foremost war that a female fights on route to her education is in her mind. To overcome the "ifs" and "buts" that society places on her head is not easy. To endure the insecurity of people around her about her declaration of attaining intellectual freedom demands perseverance of highest degree. Once these 
mental barriers are crossed, the physical ones appear scalable.

"Where's the will, there will be a way" (GTTW 13).

Part of Tash's journal entry includes a reference to a pushback against aids and handouts from the global North to the global South:

The impact these barriers to education have had on the community are; Firstly my community, in northern Uganda is allegedly still recovering from the LRA war that lasted for a period of over 20 years, i say alleged because i believe its high time people moved from dependency on donors . Its not settling in my mind that able bodied men and women can, 20 years on still be surviving on the wishful hand of donors that they have made a lifestyle out of it. some of these barriers are, dependency on aid when people could actually go to school and get employed or actually tend their farms and make an economy out of it.This has led to continued poverty and backwardness of my community which would collectively work together, to educate, inform, and mentor young children like the youth and encouraging them to go to school and actually go through with it, instead of marrying off young girls, older men spending the best of their day drinking away, fathers and mothers setting the wrong example for the children (GTTW 21). 
Other recommendations of the community members included EK. Chemorion's journal entry that details the ordeals female students underwent in some universities in Kenya as a result of them being targeted because of their gender:

World leaders reading this message should communicate to their governments that; o Ensuring security of all people in any part of the world is their mandate $o$ That security is the right of female students o And that violence against female students is violence against women and should be stopped. o And that community policing around varsities should have gender integration, o That students in all universities be sensitized to be aware of the danger zones, risky activities that can compromise their safety and times and places on and off campus while studying o That university administration take the responsibility of ensuring safe environments for their students o That businesses around universities should be inspected from time to time since students complain that the premises are hideouts for criminals. criminals should also be arrested and be charged in the court of law. o That universities ensure safe accommodation for students-particularly female students (EGBV 24).

The interview responses, which required participants to reflect on occurrences after the digital action campaigns, supplied reviews that indicate most participants could not identify 
any tangible policy changes in their local communities, while others are aware that changes may have occurred in other places; yet another respondent observed that such changes have to perhaps be self-initiated. Ifeoma replied "Ours is a conflict zone with pending conflict from more than two decades, so it is very difficult to talk about the change as it is not an easy task. But, yes, one thing that I learnt is women suffers everywhere so if they really want that their state should change for good, they have to play an active role in every development" (NR).

These journal postings, comments, and interview responses are all characterized by both a recognition of the seriousness of certain issues, and a willingness to make suggestions about how these problems might be addressed. For instance, the pushback against aids from the global North to the global South continues to come from both migrant diasporic communities, and more generally people living in developing countries. The debate between Bill Gates and Dambisa Moyo after the publication of the latter's book, Dead Aid, attracted a global audience that extended the conversation and enabled people to examine the potential consequences of discontinuing aid to developing countries (Provost, 2013). One of Gates' arguments against Moyo's position on aid is that her book and follow-up advocacy against aid have become instruments in the hands of developed countries to justify why they are cutting aid to developing countries. It is instructive to observe that none of the comments on Tash's journal entry addresses this specific concern about the complex issues financial aid is creating in her community. One could perhaps attribute this to an inadequate subject knowledge that would enable the participants to engage fully and critically in the debate. One question that arises is whether or not Tash's opinion was 
included in the submissions made by the representatives of WPc in the broader forum at which the opinions of some of the community members about girl-child education were shared. This question can be linked back to the consideration about WPc's obligation to donors who may be affected by such a stance Tash took on the subject. Tash's opinion may not be seen as ideal-as-such or conducive for continuing the relationship with the website's sponsors.

Actions

The actions code assessed indicators of steps taken by individuals and organizations to make use of the measures that are available for addressing issues raised by participants. A review of the submissions of WPc community members shows the issues that they identified and the actions they took to address the problems. In some cases, they also provide an account of steps taken by other people and organizations in this regard.

Sharontina's comment on Mukut's journal entry about an incident of street sexual harassment is an example of this practice:

I remember an incident, a brutal act, several years ago in Chennai, Sarika a college girl was dragged by her dupatta by the men in an auto and finally fell and hit her head on the stone and took the last breath. That was when the term eve-teasing became such a known phrase here and was talked about everywhere. I have experiences 
where I have hit a guy with my shopping bag, pulled his shirt and knocked him down during my college days. The moment I did this the crowd gathered to take the chance. So thats how it is done (EGBV 23).

'Women on the rising' wrote a journal entry about self-directed learning to equip herself for embracing the use of digital technology for advancement in her career and to support others in her network:

As for learning something, in $2008-2009$ during my National Youth Service Corp (NYSC) in Jos, Plateau state in Nigeria, I found an Information Technology (IT) Institute offering cheaper tutorials and have authorized Test Centre (Pearson) for IT certifications. I located the IT institute in Jos, enrolled for an Oracle Database Administration training and in that same year I emerged as the only lady in that institute that wrote the international exams (SQL, OCA and OCP) making a 100 per cent result in OCP and got certified in Oracle 10g Database Administration (WWTW 5).

Mukut's journal entry details an incident of street sexual harassment:

With the increasing incidences of harassment in Kolkata and other cities, and ineffective law, the women constables, have decided to 
take up the matter in their hands, and they have started functioning as undercover agents by acting as decoys in the areas most afflicted. Their stint has been successful so far with more than 60 offenders held in less than two months (EGBV 23).

Linda M. Ando comments on Rhoda Robinson's journal entry about the challenges female students face staying in school in Nigeria. Rhoda links this issue to poverty and extends the discussion to include possibility of female children not even getting a chance to start school in the first place. WP management also reported on follow-up actions after the GTTW campaign:

These submissions were then taken into consideration by the 21 youth delegates to the G(irls)20 Summit before delivering their recommendations to world leaders at the G20 summit in Moscow. Our Founder and CEO Jensine Larsen addressed delegates on a live panel during the summit to drive home your messages on the importance of girls' access to education. The reach of your voices didn't stop there! The delegates presented a communiqué to the G20 leaders with their ideas and suggestions, echoing your advice and solutions. The delegates highlighted the importance of girls' access to computer science, technology, and skills-based education programs; web connectivity; higher education; and entrepreneurship. In addition, they called upon G20 
leaders to prioritize the eradication of child marriage, which was a major barrier emphasized throughout the campaign (GTTW).

Paola's journal entry discusses the privileging of male over female children and adults in Guatemala and how this has spilled over into the labour market:

For this reason, in my work with the community library, Fuente de Saburia, we have launched the initiative to promote the comprehensive development of women. Forming in 2007, the "group of women from the library" have facilitated workshops to promote self-esteem, personal development, crafts, cooking, promotion of local products, and many others (WWTW 44).

\section{Outcomes}

This code is used to assess the outcomes of any actions that have been taken to address issues and problems raised in the forums. By way of example, WorldPulse.com provided a summary of the outcome of the EGBV campaign:

Following the campaign, World Pulse channeled your voices to major media outlets, advocacy groups, and policy makers to ensure that your perspectives were heard in the halls of power. At the close of the campaign we had a total of 153 voices 
speaking out on the global struggle against gender-based violence (EGBV).

Hilda Mary's journal entry about an organization's achievement after five years of working with girls and young women to access basic education in Uganda is another example:

Is it possible to make change under these circumstances? At MEMPROW, we have used girls voices in different ways, and we are beginning to be listened to by community members and elders as well as teachers. We are building partnerships and focusing on tracking impacts, going back to schools where we have made minor impacts as this gains us reentry. A school where we had carried out our empowerment programme was able to register two first grades for girls, the first in 25 years of existence. They wrote, to thank us and to share with us what to them was the most exciting news in a long time, and to give us an invitation to go back and empower more girls (EGBV 38).

WorldPulse.com provided a summary of the outcome of the GTTW campaign:

The campaign consisted of crowd-sourcing the voices of women and men championing for girls' rights, and then channeling your stories and solutions to the G(irls)20 Summit and major media 
outlets, advocacy groups, and policy makers ... Our Founder and CEO Jensine Larsen addressed delegates on a live panel during the summit to drive home your messages on the importance of girls' access to education. The reach of your voices didn't stop there! The delegates presented a communique to the G20 leaders with their ideas and suggestions, echoing your advice and solutions. The delegates highlighted the importance of girls' access to computer science, technology, and skills-based education programs; web connectivity; higher education; and entrepreneurship. In addition, they called upon G20 leaders to prioritize the eradication of child marriage, which was a major barrier emphasized throughout the campaign (GTTW).

WorldPulse.com's summary of the outcome of the WWTW campaign read as follows:

With your support, the WWTW: Women Weave the Web Campaign accomplished some amazing feats! A group of four World Pulse community members joined the Alliance for Affordable Internet's Multistakeholder Coalition in Nigeria; World Pulse CEO, Jensine Larsen, shared WWTW Campaign testimonies at Rightscon in Silicon Valley; preliminary Campaign results were shared by community member Iffat Rose 
Gill at the World Summit on the Information Society (WSIS

+10) in Geneva; two community members gave their

recommendation on digital inclusion at Social Media Week in

Nigeria; World Pulse CEO and community members shared key

campaign findings at workshop with top technology companies;

and World Pulse's Digital Action Campaigns Manager, Leana

Mayzlina, and community member Iffat Rose Gill, presented the

community's recommendations at the UN's Internet Governance

Forum in Istanbul, Turkey (WWTW).

\section{Expectations}

This code refers to assessments of the expectations of WPc community members about measures they would like to see put in place to alleviate the issues they identify. Participants provide submissions in their journal entries that suggest how issues and problems might be resolved. In these submissions, some members make their expectations known, while others offer recommendations. Tressa kentner's submission to the EGBV is an example of this practice:

My vision for ending violence against women is that every girl and every boy would receive the education that they need to lead healthy, fulfilled lives and that this education would lead to an 
understanding of the value of every human being regardless of gender, ethnicity or religious affiliation (EGBV 2).

Neema's journal entry discusses the UN's response to an assassination attempt on a Democratic Republic of Congo activist Denis Mukwege. She links the response to the larger and broader issue of Violence Against Women in the Congo:

United Nations Secretary General Ban Ki-moon can give the order in minutes to protect Dr. Mukwege, so why can't the order be given to end the violence against women in Congo? We have been waiting 16 years for someone to give that order. Our champion is evacuated to safety, and we thank you, but do you have another who will so selflessly take up his mantle? (EGBV 16).

Sherna Alexander's journal entry refers to issues of digital affordances and why women should be encouraged to make use of digital technology:

As women we need to look to each other for motivation and strength at times if not all the time. Digitally empowering women will not only enhance their lives it will ennoble the life of others and solidify and empower future generations of women, by the foundation we build today (WWTW 11). 
Lydiagcallano comments on Siatta's journal entry about the efforts of community organizations to empower female journalists in Liberia and ensure they have the necessary skill to tell the stories in their communities, especially after the civil unrest:

I would like to believe that Liberia will one day have an improved ICT scenario. As prices of computers and communication systems go down, more people will be able to afford personal computers and cellular phones with Internet connections. Also, your Internet connections will likewise pick up in speed while its cost will dip (WWTW 41).

Recommendations

This code refers to and assesses the suggestions of WPc community members about how to address the issues that have been identified. Across all three DACs there were many recommendations offered by WPc community members. This is a very common trend: in almost every single comment responding to a journal entry, there is at least one recommendation on what could be done better or differently, along with actions that could be taken to get redress on issues of injustice and inequality. Tressa kentner's journal entry about violence against women in the United States of America is an example of this practice: 
Cultural changes accomplished through education and economic empowerment will be the best way to end the victimization of women and girls (EGBV 2).

Tressa kentner's journal entry also reports on violence against women in the United States of America:

I believe that education is the key to ending practices that degrade women and girls. Denying education to girls is a form of violence that must stop. Education is a vital component for lifting girls and boys out of poverty (EGBV 2).

Valeria Barbosa da Silva's journal entry tells the story of a woman who was repeatedly abused sexually by people in authority at school and by police officers, even while she was homeless living under a bridge:

Welcoming the victim should be prioritized, creating a space of trust and comfort where she can share the pain that she keeps a secret. After the catharsis of pain, design a plan of action ... If her life is threatened, you must convince her to seek a refuge for victims, and submit to social and psychological care as well as the care of local health service. The action should be multidisciplinary and perpetrators reported to the police. Offer 
educational and social support for children with social service visits and monitoring (EGBV 29).

Tash's journal entry outlines the complex issues financial aid is creating in Ugandan communities, decrying the perpetual reliance of the government on aids from Western donors even after the period of the civil war that lasted more than 20 years. She recommends that her fellow citizens should be encouraged to get education and find employment opportunities or engage in agricultural farming. What is not evident in these recommendations is an awareness of the complex interconnection between civil unrest, inadequate social services, citizen apathy, complacency that is linked to reliance on foreign aids and handouts, as well as institutional government inefficiencies.

Solutions

This code refers to and assesses indications in the journal entries and comments that some identified issues have been resolved. Mitukhurana's journal entry about female feticide in India is one example:

In an attempt to limit the high rate of sex-selective abortions committed against girls in India, the government has outlawed the sex determination test (EGBV 3). 
Linda M. Ando comments on Rhoda Robinson's journal entry about the challenges female students face staying in school in Nigeria:

Geoffrey Canada, an innovative and dedicated educator created "The Harlem Children's Zone" a comprehensive educational program for children and their families from pre-school to college and includes health care services, parent education and more. Dr. Canada helped provide HOPE, EDUCATIONAL ACCESS and RESOURCES for Harlem children and families marginalized with poverty, crime and despair (GTTW 11).

Libudsuroy comments on MyrtleG's journal entry discussing youth's access to education in South Africa and the challenges they face in comparison to other African countries:

Dear MyrtleG, I love the way you essayed the solutions. One important lesson I learned from you today is the reminder that the boy children cannot be left behind. That for a gender-fair, gender-sensitive future, we need to take care of them, girls and boys (GTTW 15).

Repercussions 
This code refers to the repercussions that people have experienced as a result of taking actions in the course of civic engagement. These repercussions have been both negative and positive. Mitukhurana's journal entry about female feticide in India is relevant here:

Mitu refused to have the tests done and was severely persecuted by her husband and in-laws for her stubbornness. Finally, they locked her in a room and devised a plan. Mitu is allergic to eggs, so they baked a cake and told her it was egg-free, forcing her to eat it. That evening, she developed severe allergic symptoms but was denied medical aid until the next morning when she was brought to the hospital (EGBV 3).

LightMyWay comments on Adife Sudkivu's journal entry about her experience using the Maman Shujaa Centre in the Democratic Republic of Congo to access the Internet, and how she has gained knowledge of global current affairs through her exposure to communities outside the Congo:

The after effects or consequences of these wars are many in our rural areas, especially for the women who have suffered rape and sexual violence, young girls, and especially in the health and education sector. The vice is a lack of technology in the rural environment which would help them to express themselves and to collect information on world development, human rights, and 
laws in effect in the DR Congo, UN Security Council Resolution

$1325 \ldots$ Through the Maman Shujaa Center and this opportunity

given us by technology, the world might look to this category of

people living in the marginalized and vulnerable rural

community (WWTW 42).

Kotravai's journal entry refers to the case of a mother who was brave enough to report to the police that the father of her daughter's friend raped her 13-year-old daughter. Iffat Gill writes about how the lack of soft skills can lead to the dark side of digital literacy as experienced in Pakistan in her journal entry:

The strong need to adapt to swift changes in the technological and the personal landscape of its young population, calls for the importance to educate the young population about privacy and safety issues attached with the risks of their current behaviour. The situation has been under scrutiny since the decision of PTA to ban all unregistered sim cards and connections. It is illegal to be in possession of a SIM card if it is not registered. This, to some extent has assisted in tracking people who 'prey' on unsuspecting women, making it less difficult for them to hide their identity (WWTW 21). 
There are some quite positive reports of how changes came about after people had spoken out. The case of Tio in Uganda, as narrated by Tash (GTTW 21), is one example. Tio completed her education and became an educator, despite the challenges she faced as a young teenager taking care of her siblings, and later as a young child bride and mother. An indication of community members' awareness of the challenges inherent in virtual communication is seen in marvi82's comment on japalouise's journal entry:

We are too far from your reality to give solutions but I think you should positively look at what you can offer and what you can give (EGBV 51).

Another positive report is Rhoda Robinson's journal entry about the challenges female students face staying in school in Nigeria:

About 6 months back during a girls program I was running, I had the privilege of speaking to a number of young girls, both in and out of school. They shared their fears about not being able to get the kind of education they wanted, security in school and support from home. A young 15 year old girl shared that she had been brought from the village to the city to work for a family friend because they were 6 in her family and very little money. Most of the money she made as a house girl was sent back to the village and used to send her brothers to school. She had given up 
hope of getting an education and was already looking forward to marriage and starting her own family so she could stop being a servant. We had been able to encourage her to reconsider going to school and speaking with those that she lived with to support her education (GTTW 11).

SEE THE PRINT VERSION FOR ACCESS TO THIS CONTENT

A woman at an ICT training in Uganda tends to her baby while also learning. Image submitted for the 'Women Weave the Web 2014' digital action campaign.

The repercussions highlighted in these journal writings and comments indicate the consequences that may derive for some women under certain conditions in the cause of civic engagement online and offline. While there may be grave consequences for some, there are positive and beneficial consequences for others. Depending on the context and the 
nature of the civic work being performed, grave consequences can also turn out to be positive in the long run as the challenges that arise initially can be fed back into the issues, which will generate discussions that can then lead to solutions being suggested and actions taken that eventually result in good outcomes for the individuals and their extended communities. For example, an e-activist working on rape issue who is assaulted and makes the bold step of reporting the assault can be doubly victimized when she is asked for a bribe before law enforcement officers will address her case. This brings the complex intersection of the issues of assault and corruption to the fore, and indicates potentially where attention should be directed in future social action.

Volunteerism

This code refers to WPc community members doing work without remuneration. This work could be online on the WPc platform, or offline in local or global communities. In my previous discussion of the issue of WP management recruiting volunteer commentators on the WPc platform, I outlined the ethical dilemma of WP management recruiting community members as volunteers who are assigned specific journals to read and provide comments on. Table 32 shows the hours WPc members spent on the website and the level and extent of their sub-committee membership. Details about these connections are available in Appendix 14. 
Questions 9 and 12: How many hours a week do you spend on

WorldPulse.com? * Are you a member of any of the subcommittees on WorldPulse.com?

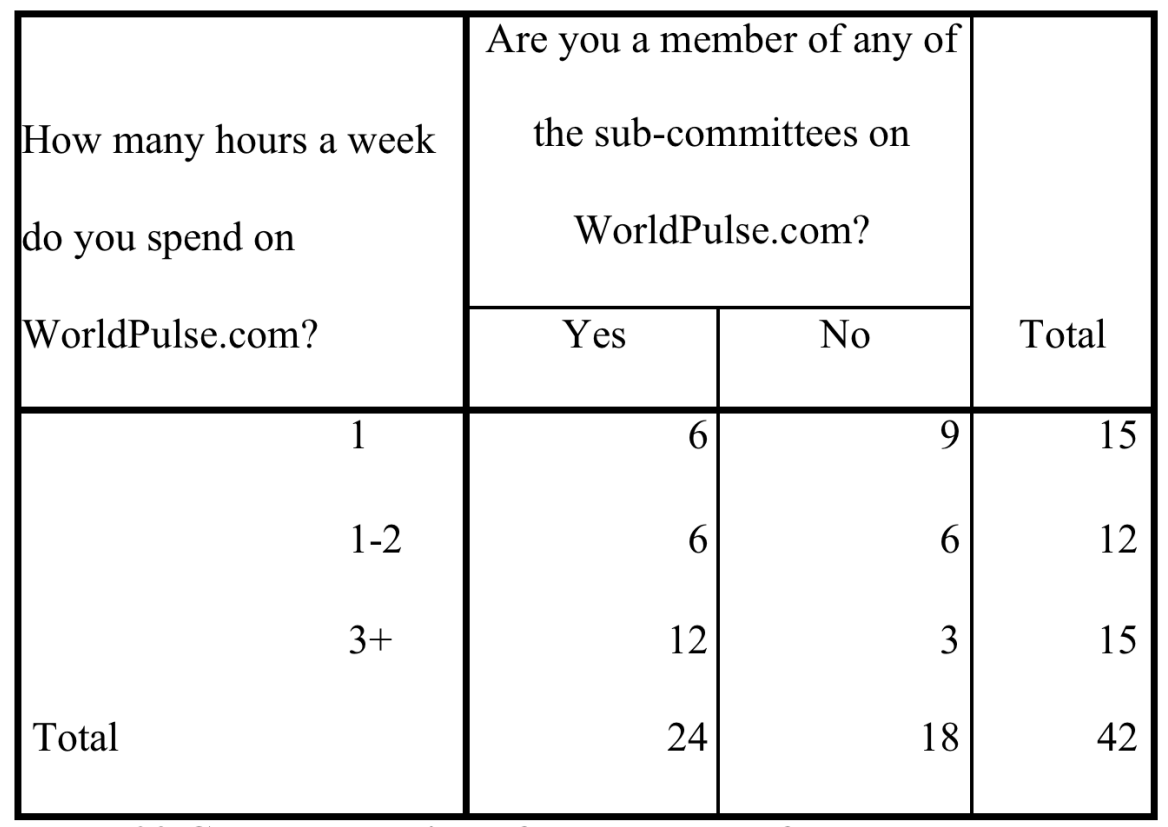

Table 32 Cross-tabulation of the number of hours survey respondents spend on WorldPulse.com in comparison to their role as sub-committee members, which is voluntary work.

Table 32 indicates that 24 of the 47 survey respondents are sub-committee members on WPc. This 24 represents a significant $50 \%$ without accounting for the additional survey respondents who may not be sub-committee members but also make voluntary contribution through the publication of their 'Voices of Our Future' articles that are unremunerated. Out of these 24 people, 12 spend more than three hours per week on WPc. 
An explicit reference to the task of assigning journal entries to volunteer community members appears in KOMoore's response to siatta's journal entry in the WWTW campaign:

I am so fortunate to have been assigned as a reader for your written story. It is so important you are making visible the challenges and barriers to internet access for women in Liberia. It sounds like there are also some things to remain positive about; there are women who did access the journalist training, fiber-optic access has been built and research is supporting the need for access. Building on these factors and encouraging others to join your campaign will lead to further change. Do not be discouraged! Keep writing! I can think of no greater issue than making the world safer for women. It has been important to me to understand what is going on in countries like Liberia. Thank you for educating me. Sometimes we still struggle in the USA too. I will recommend to World Pulse to promote your story. Warm Regards, Karen (WWTW 41).

The interview data back up the observation of a common and communal practice of voluntary work appearing in the journals from the digital action campaigns and online web survey data. Interview respondents have formal roles on WPc that they occupy on a voluntary basis and these roles carry varied responsibilities. Chika writes "I am also a community listener, and recently I've been selected as a community champion (thus my 
new duty will be co-moderating the group on economic empowerment)" (NR). Amara replied "I am a member of the World Pulse community advisory board... and community champion [for] gender based violence group" (NR). Ogechi writes "I share with other women how to use their personal profile page...I show the Resource Exchange and Training tab to find opportunities...I show the Member Directory...I show them how to engage, to welcome others, to develop an active presence on World Pulse” (NR).

The interview data also showed that all respondents who have participated in the "Voices of Our Future' training - either as correspondents or editorial mentors- were not paid for their participation. The editorial mentor role is voluntary as well. While the VOF training is offered free and arguably not expected to be remunerated, one would expect the articles that the correspondents produced, and most especially the ones that were published, to have been remunerated. By extension, the willingness of WPc participants to work without remuneration can also be linked to the awareness that they need to build social capital with other members on the site, as well as the site management, in order for them to assess the other opportunities - such as training, travel opportunities, speaking engagements, representing the website locally and globally offline - that are available to members of the platform.

Authentication

Authentication emerged as an essential ingredient for embedded e-activists, especially for the purpose of knowing diverse information about the people they are interacting with; 
there is an extension of this knowledge to connecting the people with the issues they advocate about and why they focus on those particular issues, perhaps in relation to whose interests the people are representing. The online web survey asked to know the degree of importance of some demographic information of WPc community members when participating on the platform. The data showed the percentage of participants that would like to know about other participants' race (29.3\%), gender (53.7\%), age (26.8\%), profession (63.4\%) and level of education (31.7\%). See Appendix 2 Question 13 for the details. Tables 14 a to $14 \mathrm{e}$ show the breakdown of the demographic information. A detailed breakdown is available in Appendix 2.

\section{Question 13: Which of the following information would you}

like to know about other participants when engaging on

WorldPulse.com - Race

\begin{tabular}{|l|r|r|r|r|}
\hline & Frequency & Percent & Percent & Percent \\
\hline Valid 1 & 12 & 29.3 & 100.0 & 100.0 \\
Missing System & 29 & 70.7 & & \\
Total & 41 & 100.0 & & \\
\hline
\end{tabular}

Table 14a: Information survey participants would like to know - Race

Table 14 a shows that $29.3 \%$ of the respondents would have liked to know the race of other participants, while $70.7 \%$ did not. 
Question 13: Which of the following information would you

like to know about other participants when engaging on

WorldPulse.com - Gender

\begin{tabular}{|l|r|r|r|r|}
\hline & Frequency & Percent & Percent & Percent \\
\hline Valid 1 & 22 & 53.7 & 100.0 & 100.0 \\
Missing System & 19 & 46.3 & & \\
Total & 41 & 100.0 & & \\
\hline
\end{tabular}

Table 14b: Information survey participants would like to know - Gender

Table $14 \mathrm{~b}$ shows that $53.7 \%$ of the respondents would have liked to know the gender of other participants, while $46.3 \%$ did not. 


\section{Question 13: Which of the following information would you}

like to know about other participants when engaging on

WorldPulse.com - Age

\begin{tabular}{|l|r|r|r|r|}
\hline & Frequency & Percent & Percent & Percent \\
\hline Valid 1 & 11 & 26.8 & 100.0 & 100.0 \\
Missing System & 30 & 73.2 & & \\
Total & 41 & 100.0 & & \\
\hline
\end{tabular}

Table 14c: Information survey participants would like to know - Age

Table $14 \mathrm{c}$ shows that $26.8 \%$ of the respondents wanted to know the age of other participants, while $73.2 \%$ did not. 
Question 13: Which of the following information would you like to know about other participants when engaging on WorldPulse.com - Profession

\begin{tabular}{|l|r|r|r|r|}
\hline & Frequency & Percent & Percent & Percent \\
\hline Valid 1 & 26 & 63.4 & 100.0 & 100.0 \\
Missing System & 15 & 36.6 & & \\
Total & 41 & 100.0 & & \\
\hline
\end{tabular}

Table 14d: Information survey participants would like to know - Profession

Table $14 \mathrm{~d}$ shows that $63.4 \%$ of the respondents wanted to know the profession of other participants, while $36.6 \%$ did not. 


\section{Question 13: Which of the following information would you}

like to know about other participants when engaging on

WorldPulse.com - Level of education

\begin{tabular}{|l|r|r|r|r|}
\hline & Frequency & Percent & Percent & Percent \\
\hline Valid 1 & 13 & 31.7 & 100.0 & 100.0 \\
Missing System & 28 & 68.3 & & \\
Total & 41 & 100.0 & & \\
\hline
\end{tabular}

Table 14e: Information survey participants would like to know - Education

Table $14 \mathrm{e}$ shows that $31.7 \%$ of the respondents wanted to know the education level of other participants, while $68.3 \%$ did not.

The percentage of survey respondents showing interest in gender issues at $53.7 \%$ indicates that the presumption that WPc is essentially a space for the discussion of issues pertaining to women is not entirely justified. An observation could also be made about the high percentage of people wanting to know about other participants' professions, at $63.4 \%$. The low percentages indicated for race, age and level of education suggests that participants do not consider this demographic information important for determining the authenticity of participants or the participants' claims. Overall, this desire on the part of participants to acquire demographic information about other participants indicates that the issue of the authenticity or otherwise of online identity is an important consideration for e-activists. By 
extension it suggests that the level of online civic engagement is to a certain extent predicated on processes of verification and authentication of claimed identities.

Value

It appears WPc participants place a high value on their membership of WPc and their participation in the digital action campaigns and VOF training program. Interview participants express this value in different contexts, including what it enables them to do during and after the campaigns. Nkem writes "At first my experience was very good. My expectations was to develop a relationship of sorts with people I mailed back and forth with ... I was not on WP to get help.i was on to help others" (NR). Emeka replied "Participating on worldpulse has increased my knowledge about certain global issues especially among women, it has improved my empathy and listening skills" (NR). Chika writes "On a practical level it motivated me to volunteer at a local community [name], where I taught about social media (mainly blogging). But I believe the larger effect was on a mental (theoretical) level" (NR). Uloma replied "They have made me more aware of the technological and digital literacy, and how without it, members of the community can be left out of important, current and meaningful conversations. It has also made me more aware of the importance of one's personal experience on their view of the world" (NR). Amara responded "Once our [community] women's picture was used as the face of Web we want campaign and it also links me with other [nationals] and women elsewhere" (NR). Ifeoma writes "I would talk about my VOF in which I was empowered how to make better use of technologies and other empowerment tools to make the storytelling experiences of 
the affected population more powerful. I also became more motivated by listening to the stories of other women and get inspired to work more and specifically look for many other issues that the women at the global level were focusing one and I was not mindful" (NR). Ogechi replied "The campaigns are a rallying point, a discussion point, and a unifying vehicle as we all participate and share our ideas" (NR).

Intrinsic in the interview respondents' accounts of these values is a sense of empowerment undergirded by a sense of belonging as part of an online community of social activists who are able to use their skills and networks both online on WPc and also in their extended offline communities.

\section{Collective Voice}

Interview participants consider WPc a global space that affords them an opportunity to contribute to community development at both global and local levels using tools that allow them to combine online and offline activities towards achieving their goals of making social change. Emeka writes "I'm glad to be part of a movement that encourages better communication and access to information" (NR). Chika replied "I believe that during the VOF program, I did send the message that there can be young religiously committed females in "third-world" countries who are open, logical, and thrive to change the world" (NR). Uloma writes "I love being able to do this! I love communicating with people around the world who have a specific focus that I share with them. I feel that I am helping others and helping to create positive change" (NR). Amara replied "It also afforded me the 
opportunity to strengthen other violated women's voices" (NR). Ifeoma writes "It is always enriching and helpful to connect with the fellow sisters via WorldPulse.com and know about them. The experience is quite different from social networking websites where people update their status and posts leisurely. This medium is purely professional and meaningful as people not only talk about their sufferings but also successful stories against all odds. So, using internet to access WP gives me immense pleasure to relate to the global women and be a part of them and their lives through this unique powerful medium" (NR).

When asked if they considered themselves a part of a global community through their involvement in WorldPulse.com, interview participants responded in the affirmative. Chika writes "I consider myself so and I am very proud to be part of this global community" (NR). Ifeoma replied "Yes, very much indeed! The day I joined WP, I found myself lucky enough to become a part of this amazing world of global networking of unique storytellers, without any professional training. But, their experiences and the power to speak out makes them global leaders and powerful communicators" (NR). Funanya writes "Yes sure. I believe in world pulse a lot and I believe some one somewhere read about what happens locally which make it globally" (NR). Ogechi replied "Being connected online is huge in this desperate part of the world. To be able to speak my/our mind to a WorldWideWeb audience has opened up a world of love, solidarity, and support for those us [women] who have taken hold of the virtual microphone. To travel the world daily without a ticket or a visa; to find I've visited the home of women in South America, Australia, Europe, the U.S., Canada, India, Palestine, and of course all the other countries in Africa, is amazing to me. To receive encouraging comments from my sisters is like they have come to visit me in my 
home. We are sharing our hearts and giving big virtual hugs. And once in a while we are blessed to find each other in person somewhere in the world, and we just take up where we left off online. It is wonderful" (NR).

While some interview respondents clearly expressed that they feel like their contributions link to a collective representation on WPc, others were not too clear about this, although they highlight the specific input they made during DACs and VOF training that may or may not have been included in the final recommendations in WPc reports about the digital action campaigns or citizen journalism training program. Most participants expressed ambivalence and a high level of uncertainty about the inclusion of their recommendations in the final campaign report. Chika writes "I think there can be a kind of yearly scholarships to one-to-two-maybe three-maybe five pioneer women, who have ideas and want to do something but cannot afford it. It doesn't have to be financial, maybe it can be academic for instance. But aside to this I believe WorldPulse is already doing an outstanding job-a job that governments worldwide are running from!" (NR). Amara replied "They should be organising offline campaigns in various cities too" (NR). Ifeoma writes "It would be great, if WP could support its members on the offline medium so that they would be able to have the amazing interactions with their community leaders and spread the experiences of the fellow sisters directly, may be through the powerful use of virtual media. Although, it is great to connect with the people on offline mode and learn from them. But, it would be doubly relevant and significant to disseminate the same in-person" (NR). Funanya writes "Breaking the Silence project" (NR). Ogechi replied "I think the WorldPulse platform is okay. But I do wish that World Pulse could do a visit to [country] 
to see us and get to know us personally" (NR). Perhaps a way to further strengthen this discourse of collective voice is for WPc to assess other work its members think it should be doing, work that would arguably make it more representative for its community members.

\section{Conclusion}

The responses of the interview participants indicate the importance, to the community members, that the broad and diverse needs of the community members be represented and articulated. By extension, this representativeness also affects the relevance and outcome of the works that embedded e-activists do online and offline. The level of journalistic practice on WPc is extensive. The issues WPc community members discuss and comment on often extend the agenda beyond what has been set by WPc management prior to each campaign. Community members also identified measures that they think need to be taken in addressing the issues that they raised. These measures are both individual and institutional, and also include actions that could be traced to policy and governance changes in local communities. The actions taken by individuals and groups in using the measurable tools they have to address problems is well documented in their journal writings on WPc. There are detailed reports of the outcomes of digital action campaigns and community members' activities during and after the campaigns. WPc's embedded e-activists also discussed their expectations about the issues and the measures that can be taken to address the challenges they face. 
The community members also offered recommendations about the processes of articulation, discussion and engagement on the platform. Volunteerism is at the heart of the work of embedded e-activists, and this engagement often occurs simultaneously online and offline. These activists also work with WPc management online, offline and behind the scenes to authenticate the identity of participants, as well as the stories that are produced and circulated in the journal entries. They place considerable value on their civic engagement activities and in this respect, require some clear demographic information about the people with whom they conduct their civic activities. The communal sense of belonging extends to the understanding that WPc community members offer a collective voice on issues that they discuss and write about in their journal entries.

The three categories of analysis employed in this critical analysis and discussed over the last three chapters have enabled an assessment of, and provided evidence of the extent to which, WorldPulse.com and the civic engagement and experiences of its community members constitute a form of public sphere and public sphere activity. Three evaluative regimes or models were employed in this process. Habermas' model addresses issues of communication practices, the people engaging in the practices and, to some lesser extent, the platform on which the communication occurs. DiMaggio and Hargittai's model assesses the multiple facets of inequality that can accrue as a result of limitations from technological access, but even more importantly looking beyond access to get at the challenge of inequality among those who already have access to the Internet. The model was useful for getting to the core of challenges in soft skills, especially technical knowledge of the topics 
being discussed and the language skills necessary to engage in deliberations in virtual public spaces.

The civic engagement typology developed organically while the data was being collected, coded and analyzed, as there were indications of gaps in the two models from Habermas and DiMaggio and Hargittai. The civic engagement model for embedded e-activists is useful for assessing the agents participating in and the elements of civics that are not confined to one space - either virtual or physical - but rather overlap and intersect in the course of social action. The categories of analysis identified in the model function to facilitate the work of embedded e-activists who work simultaneously online and offline in the civic engagement space. The analysis indicates a need to further build the capacity of women for engagement in the public domain, as access to technology is still limited in some communities, while those with access would further benefit from advanced technical knowledge.

The following chapter provides a discussion of the analysis conducted over the previous three chapters, with a specific focus on answering the questions set out at the beginning of this research. The research questions are: 1) to what extent does WorldPulse.com function as, and constitute, a site or space of public sphere activity? 2) to what extent do activities on WorldPulse.com translate into social, cultural or political actions, such as changes in policy and mode of governance in the members' communities? 3) how can we characterize and organize the different contributions of participants on WorldPulse.com? 4) to what extent does the move to a virtual or digital public sphere require a reformulation of the notions of 
identity and identification, which is a critical issue for analyzing and understanding the roles, functions and parameters of the public sphere? The overriding thesis statement for the research asserts that WorldPulse.com has the potential to function as a micro public sphere for marginalized groups, especially women. The thesis then applied the research questions to assess this assertion with the goal of using dialectical triangulation of theories, methods and data to examine the thesis statement. Chapter Nine provides an extended discussion of the thesis statement that is based on the data analyzed in relation to the theories and scholarship introduced in previous chapters. 
Chapter Nine: An Analysis of the Themes Identified in the Data from WorldPulse.com

Introduction

In this chapter, I will conduct an analysis of the themes that have emerged from the data. In the first section, I discuss the ability of WorldPulse.com to serve as a global public sphere based on information that emerged from the data while assessing it against Habermas' criteria of the public sphere. Across all the WPc members who participated in the interviews, there was evidence supporting Wellman et al's (2001) 'supplement hypothesis' that the use of new media for civic duties supplements, rather than replaces, citizen's use of old media for communication. Dahlgren (2005) and Papacharissi (2002) have pointed out that offline identities act on online identities, but that the reverse also happens. However, 'old sites' are not necessarily much more than 'empty performatives.' Old sites are important at times, but they are not the best space for the kind of public sphere activity that challenges power.

The second section of this chapter discusses the five broad forms of inequality DiMaggio and Hargittai identified. This discussion situates the five broad forms of inequality in the context of more recent data - from the DACs and also external to WPc - indicating that these forms of inequality persists in various degrees across all demographics and regions of the world. Section three makes connections between the characteristics of embedded eactivists and offers a model for assessing the work they do offline and online in a visual format while discussing and indicating the four pathways that embedded e-activists use 
when engaging in social action. Section four revisits the roles and functions that validate, but also problematize, the position of WPc as a community that is representative of women's interests.

WorldPulse.com as a Micro Public Sphere

WorldPulse.com serves its community as a micro public sphere and articulates the discussion and deliberations through to the macro public sphere by using offline engagements on larger platforms such as the TEDx stage, United Nations conferences and other industry forums. This section answers the first question set out in this research project. The question is:

- To what extent does WorldPulse.com function as and constitute a site or space of public sphere activity?

The public sphere is still very much an ideal and even more so utopian in its expectations, even after the revisions by Habermas and other scholars. WorldPulse.com constitutes, and functions as, a kind of alternative public sphere space; what we can term a micro public sphere site in Fraser's terms (1992), where the prescriptive ideals outlined by Habermas are replaced by more flexible and pragmatic criteria. By way of example, the exchange and critique of logical reason and ideas are not commonplace on WPc, but the platform aids in facilitating discussions among its members and between its members and other small pockets of publics. Civility is extensive on WPc and this ties in with Blumler and 
Gurevitch's (2001) discussion about Habermas' criteria for the space used in facilitating deliberations.

The DACs and VOF training program appear to be the two major tools that WPc uses to activate the community of women and facilitate discussions. Most interview participants see these tools as being worthwhile communal efforts for drawing attention to their causes and plights; however, they also point out that the website could be more for its members and other women globally. Ugarteche (2007) has drawn attention to the need for an acknowledgement of the existence of national interests with regard to the notion and activities of global public spheres, although Volkmer (2014) suggest that public sphere activity has become more global due to the effects of transnationality and cross-border migration, and the spread of digital media. However Ugarteche's (2007) contention that national interests still dominate most global public sphere sites is echoed in some of the interview material.

Prior identification of issues and setting agenda as cited by Habermas is evident on WPc, although an extensive and close revision of the journal entries and comments indicate that the members add items to this pre-set agenda. Most of these added items go unaddressed in the immediate campaign; it is not clear if WPc management take on the added items offline for new campaigns. Fraser's charge about inequalities driving discussion rather than being bracketed is not manifested in WPc community experiences. Consensus is another problematic assumption, since there is no evidence of a site-wide mechanism on the site for members to be consulted on what items should be on the agenda. WP staff present an 
agenda in which it issues a call for action based on the assumption that the WPc membership would find the topic relevant for discussion. WPc does function well in circulating the stories of most of its members, and by extension gets them a degree of recognition that may not be available to them on other platforms. These practices affirm a sense of the undeniable humanity of the people and how this humanity is denied on other platforms.

Most commentators and journal writers do point out the diversity of issues and needs, but these issues appear to just lay out there with no action likely to be taken. The expectation that those involved in the discussion of issues would be a part of setting the agenda also did not feature on WPc, at least not in the front end of the forum. There is no indication either that the discussants are consulted prior to the agenda being set. It appears that most topics are modeled after events that have been pre-determined by forces external to the forum. For example, the EGBV and GTTW campaigns are linked to two United Nations international days. There is no indication that the topics discussed are burning issues that WPc members identified in the course of community consultation. This observation is backed up by information from one of the WP staff that participated in the interview. She indicated that the website is just now (as of 2015) developing a strategy for determining the subjects to address in the digital action campaigns. Perhaps WPc should introduce a space where members can propose topics for discussion and these can then be assessed to determine which ones to address.

Agency, Rationality and Emotionality, and Exclusive Inclusivity 
The website culture on WPc is markedly different from other online forums. On WPc, there is little evidence of concerns about flaming and it appears there is minimal need for a moderator, other than perhaps when such a position is needed for technical support, as opposed to being a 'referee' among warring parties. Autonomy is still a challenge due to the site's links with donors who have ideological agendas and the links of individual community members with other NGOs, community organizations, business and government agencies whose interests they represent indirectly. However this lack of autonomy does not affect the website's ability to deliver its programs and still represent its community members on the issues that they have identified.

Exchange and critique of moral-practical validity claims is very scarce on WPc. There appears to be a general community code that borders on agreement among and between members. Very rarely do other members challenge positions of other community members on some issues being discussed. The community members' relations with WPc management is always subject to factors such as the latter's higher levels of socio-cultural and political capital, which limits the level of leverage that community members have on the platform; an issue Foucault and Bourdieu draw our attention to. The sensitivity of the subjects and issues being discussed and the general absence of opposing views on the positions expressed by the discussants is another factor. It appears that most members hold similar views on some issues; hence, consensus is easily achieved. This is not so much to do with the gender of the participants, as it is to do with perhaps their ideological positioning. WPc is arguably composed of mostly centre-left leaning pro-choice members. 
On the rare occasions when seemingly conservative views have been expressed, such views are very quickly overtaken by the overwhelming majority of liberal-leaning members whose request for reasonability from other members is underpinned by an appeal to emotion and references to personal experiences and encounters with the issues rather than rational-critical critique of the issues and the positions of the discussants.

Moreover, critical-rational discourse is limited on WPc; reason and rationality conflict and compete for manifestation. For discursive inclusion and equality to manifest on WPc, one has to consider how it may be affected by other factors such as autonomy and exchange and critique. One aspect of WPc's role as a public sphere space is the format of the discussions: members make submissions in individual journals and other members comment on those submissions. Although these journal submissions appear as individual write-ups, in similar format as a news item on a newsfeed for instance, they are still linked to the original issue WPc placed on the agenda. The representation of WPc as an absolutely inclusive and positive space is problematic, especially due to the sensitivity of some of the issues and the closeness of the participants to the subjects being discussed. While some members are able to openly engage with certain issues and topics, others are reluctant to do so, either because they find the sometimes graphic details of the events jarring or have experienced some of those issues personally and still find it difficult to confront them in such an open space.

Being an inclusive space is a necessary feature for a platform like WPc to thrive, especially considering the victimization that some of the members have been subjected to in other spaces - both offline and online. However, this requirement for positivity and inclusiveness 
has the potential to stifle debates and the articulation of different positions and perspectives. Inclusivity and equality in this context seems to stand in opposition to the desire for critical-rational debate. These competing ideals then raise the question of to what extent does the Habermasian template have to determine the situation in which (a) technology has changed the nature of the public sphere and public sphere activity, and (b) a particular site (WPc) has its own specific situations and issues and has to 'take care of these' if it wants people to contribute. The simultaneous manifestation of these two criteria needs ongoing negotiation and a delicate balancing act among WPc members. An example of this is discussed further below in the journal entry to the EGBV campaign from Tessie suggesting 'a woman was asking for it' and the accompanying comment that attempted to criticize that journal entry. Equal access is a utopian ideal, considering the stark differences between technological demands and soft skills between members in the global North and those in the global South. However, it is important to note that any form of access, no matter how restrictive, is good access and any opportunity to bridge the inequality gap is worthwhile.

Reflexive Engagement, Empathetic Discourse, and 'Natural' Sincerity

Taken together, the manifestation of these three criteria as observed on WPc further indicates the site's uniqueness - as a forum for women engaging in civic activities - when compared to a range of online discussion sites. There is an overwhelming and perhaps excessive occurrence of reflexivity, ideal role taking, and sincerity on WPc. It would be worthwhile to see how different the site would be if its membership had a slightly different mix of gender representation and identity - in terms of members having the benefit that 
comes with the cloak of anonymity and power dynamics (linked to global North versus global South positioning of members, the economic imbalance in the context of global South members seeking opportunities and referrals to training, attendance at international speaking engagements, support for setting up NGOs and non-profit organizations from global North members). WPc offers a global access, but one should remember that it is only one of many diverse public spheres, even in the area of the concentration on issues for women empowerment, training and advocacy on women's issues. Other similar spaces for such include the Global Press Institute (with the Global Press Journal).

Reflexivity is in excess after the fact, as observed in the interview responses; however, there is not much reflexivity occurring in the DAC journals or comments. WPc members not reflecting in the moment while the campaign is ongoing presumably feeds into some of the rules of engagement on WPc. In a sense, those rules are derived from the very difficult situation in which members find themselves; as Foucault draws our attention to the uneven power relations between the forum owners and members. There are competing imperatives to consider in this case, including an ongoing investigation of the fundamental purpose of public sphere activity and examining whether its primary aim is to adhere to an abstraction or find a way to change things.

Ideal role taking is extensive on WPc and its excess appearance further highlights the uniqueness of the site, perhaps due to the membership composition, the nature of issues being discussed, the kinds of experiences the members have and the forms of engagements. Reflexivity and ideal role taking could be complementary, but also in opposition when in 
most public sphere engagements. Ideal role taking calls for empathy, while reflexivity needs people to interrogate their own situations and positions. These two criteria do not often go together; even more so when contextualized against the subjects that participants are discussing.

Sincerity is difficult to gauge in an online forum generally, perhaps as it is equally difficult to gauge offline, but it was somewhat easier to assess on WPc because some members use screen names that link to their offline identity. Information in the DAC journals also often points to real life experiences. Combined with information from the survey and interviews, one can make a case for a sizeable amount of sincere representation of individuals and issues. The opportunity to attain a fairly large amount of sincerity is no doubt owing to the nature of WPc, as well as the decision to utilize data triangulation for this project. Despite all the limitations encountered, I would argue that sincerity is still a required performative on WPc. A form of communication defines the public sphere; the form of communication occurring on WPc does not conform fully to the expectations of Habermas' public sphere, but it nonetheless appears to serve the purpose of the owners of the website and quite a reasonable number of the WPc community members, based on information from all the data sets assessed in this research.

The Digital Divide and Digital Inequality

The suggestion by DiMaggio and Hargittai (2001) that attention should shift to digital inequality, and the observations made by Liff and Shepperd (2003) about technical skill 
inequality in the U.K., are valid, but the data used in this project indicates that social science researchers still need to pay attention to the question of the digital divide. Most especially, the interview data indicate the need to assess the digital divide. Most participants own multiple devices, but access is still a challenge in some places - including the Appalachians in the United States of America - while soft skills may have improved in other places. Some WPc members are doubly challenged - in keeping with the observation by Alzouma (2013) in the case of rural-urban divide in Niger - because of their limited and restricted technological access. This takes the form, for instance, of periodic and systemic Internet monitoring and control in India, China or Egypt, combined with the limited language skills, say for members in South America or the Democratic Republic of Congo. A very recent example is the blanket Internet gag that was placed on Egyptians during the attempted coup in that country in July 2016. Another factor is the subject knowledge required to engage in discussions (Emden and Midgley, 2013; Tully, 2013). These issues further highlight the limitations of the Internet as a site for public sphere activity and civic engagement.

In this section I attempt to answer one of the questions set out in this research project:

- How can we characterize and organize the different contributions on WorldPulse.com?

Respect and mutual agreement is often quickly established, or at least performed, among WPc community members, and there appears to be an unspoken code of conduct that is 
predicated on the site's terms of service. The format of the discussions, the identities of the discussants, community members' personal connection to the issues and the sensitive nature of the discussion topics are some of the factors that affect this negotiation.

The Persistent Digital Divide, Capacity Building, and Digital Affordances

For technical apparatus, all interview participants own multiple devices, but access to the Internet is still a challenge for some people. Penetration has increased in some areas but other restrictions exist due to accessing on a mobile versus tabletop device. This restriction is connected to variation of use, yet challenges inequality in skill since skill becomes moot if technical apparatus is limiting. The interview data indicate that full ownership of technology enhances access and participation in the public sphere; likewise, the writings assessed in the journal entries and comments. Gordon's (2002) and Volkmer's (2014) studies of mobile phone use make a similar case that connects autonomy, technology and agency. A 2015 report emphasizing the need for digital inclusion further buttresses this fact (Stephenson, 2015). This report makes a case for digital inclusion across all age groups and identifies web access, skills and motivation and trust as factors to consider in making digital inclusion a priority. A bigger challenge, perhaps more so for individuals than technology providers, is the 'big brother is watching' surveillance culture discussed by Boeder (2005) and perhaps much more broadly alluded to by Foucault in his seminal discussion of surveillance through the concept of the panopticon. If many individuals perceive that their privacy could be jeopardized, they are more likely to limit their use of 
Internet technology even when there is evidence indicating the multiple benefits of using the technology.

For inequality in skill, most WPc community members claim to be representing their own interests. Skill is closely related to autonomy. Most interview respondents appear confident that their purposes for going online are being met, but again accessibility problematizes the continuity of completing the tasks of representation. The physical letter writing practices Gring-Pemble (1998) assessed are similar to the electronic journal entries being produced on WPc. There is no doubt that this practice has evolved over the years, as more people now go online to use electronic journals for what would otherwise be private, individual reflections. However, inequality in skill, which involves inability to grasp the technicalities of the subjects being discussed as well as language deficiency, means that some people are still not able to participate in this practice. The case can also be made that there are many people who have the technical knowledge and language skill but choose not to participate. Inequality in variation in use of technology is linked to soft skills. This is a key factor in the context of knowledge and understanding of the issues being discussed and the ability of WPc members to participate gainfully in the deliberations.

Digital Inequality and Socio-Cultural Inclusivity

Autonomy of use appears commonplace, as most interview respondents suggest they participate voluntarily, yet some of these people are members of extended groups and communities online and offline and they would work to represent the interests of these 
groups and communities. From all the data sets, it is obvious that women have autonomy of use in terms of access to digital technology, but there is still a gap in social support generally. In places where digital divide persists, this is compounded by digital inequality vis-à-vis a lack of soft skills ('Women’s Rights Online: Translating Access into Empowerment', 2015). The operation of a digital public sphere, no matter how successful on its own terms, does not necessarily equate to political action or change. Bourdieu's (1989) notion of the 'Gricean Co-operative Principle' asks how we evaluate and understand public sphere sites and activities, and the work and projects they carry out in terms of public sphere sites circulating and disseminating certain stories, ideas and voices, and critiques that constitute a set of discourses as critique of power and power differentials. In a sense, power has to take some kind of notice and provide some kind of response to these stories; after all, in a sense, these stories constitute a kind of scandal that threatens the performances of power - they are to some extent 'little events' that have the potential to make people more reflexive about how inequality is naturalized and normalized.

In addition, there is a way in which those stories, once put into circulation, make it all the more difficult for power to persist in its various regimes of dehumanization - racism, sexism, the disregard of the other as worthy of concern or significance. Butler's Giving an Account of Oneself (2005) and Precarious Life (2006) pick up on this point: representations of the other (even self-representations) make the other recognizably human-like-us. These are powerful representations that produce empathy, but they also undermine the various often unspoken - arguments or discourses that naturalize the other as 'less than human'. What these stories do is provide the other with a face, a human face (Levinas, 1985). 
Additionally, one of the more important aspects of WPc and others like it is that it enables people to share their stories, and to understand that there are people 'like them' who are struggling against the same kind of challenge. By way of extension, one of the things WPc provides is knowledge and advice; people can learn how others coped and dealt with issues. Social support intersects with autonomy of use but it appears a sense of indebtedness towards WPc management and community members develops for those members who have accessed such ongoing support. This feeling of indebtedness might also restrict members' autonomy of use of the platform although it also increases the sense and perception of being a part of a global community of likeminded people.

Embedded e-Activists: The Intersection of Online and Offline Advocacy

Dahlgren's (2012) notion of 'civic intellectuals' is broad enough to accommodate embedded e-activists. By way of example, reflexivity, as understood by Dahlgren (2012) and Volkmer (2014), is evident within, and even central to, the communication practices and culture of the WPc community. We made the point that the culture on WPc is, first and foremost, predicated upon an acceptance of, and a willingness to sympathetically engage with, otherness. We need to break this issue down somewhat, however, if we are to establish a valid and viable connection between the idea of reflexivity understood or produced as a kind of intellectual abstraction, and reflexivity as an everyday cultural value and determinant of communication practices. Precisely because the members of WPc come from diverse and often non-Western backgrounds, this means that their values, practices, experiences, world views and forms of cultural literacy will be to some extent different 
both from each other, and from what we might term the 'dominant' culture of the West. In a sense we could argue that the socio-political underpinning of the WPc site is a recognition that otherness is a political issue; indeed, to some extent it is their main political issue, since the forms of inequality (social, cultural, political, economic) that they seek to address are based on and accentuated by what we can call, after Bourdieu (2000), certain naturalized forms of symbolic capital, domination and violence. What follows from this context and culture, then, is that forms of reflexivity about or with regard to symbolic domination are always part of the ways in which communication is understood and practiced; in other words, we can say that the culture on WPc is necessarily and ideologically reflexive.

How does this relate to the issue of 'civic intellectuals'? In order to develop this connection, we need to look to Bourdieu's work in this area. Schirato and Webb write that:

For Bourdieu, the extent to which agents can attain knowledge of, and negotiate, various cultural fields is dependent upon, and is explicable in terms of, two epistemological types. The first is the practical sense or logic of practice ... while the second involves a reflexive relation to the habitus, the demands and influences exerted by cultural fields and one's own practices within those fields (2003, pp. 539-52)

They go on to suggest that Bourdieu's concept of reflexivity is field specific, but generally tied to and practiced within those fields that dispose the habitus of the field to 'think against 
itself; or at least to think against that which has achieved the status of doxa or symbolic dominance. They also argue that, for Bourdieu, the fields that approximate these conditions are of a scientific or intellectual character.

Bourdieu seems to reserve reflexive knowledge for the field(s) of science, but ... he is not altogether consistent in this regard, identifying reflexivity, at different times, with art, literature, history and the intellectual field. And in fact there is no reason why the conditions he sets for the institutionalizing of a (potential) reflexive disposition, that is, the systematic exploration of the 'unthought categories of thought which delimit the thinkable and predetermine the thought' ... should not be available to any number of fields. If the key is to encourage and reward a reflexive habitus through the institutionalizing of a reflexive disposition in 'mechanisms of training, dialogue, and critical evaluation' ... then any or all of the fields that fulfil this criterion have the potential to produce reflexive knowledge ... and reflexive subjects (Schirato and Webb, 2003, pp. 539$52)$.

WorldPulse.com and Political Action

The next question that will be addressed is: 
- To what extent do activities on WorldPulse.com translate into social, cultural or political actions, such as changes in policy and governance in the members' communities?

Measurable action comes to fore through members' self-reporting on WPc and also at conferences during speaking engagements facilitated by WPc and its partners. Interview respondents do not see much of policy changes in their communities that can be directly traced to activities on WPc, but do acknowledge that this may be happening in other places and other ways they are unaware of. WPc needs to put better mechanisms in place for reporting on any policy changes and measurable action they can directly link to their DACs.

Authenticity is managed through member-to-member interactions and management-tomember backend work. There is an acknowledgement of an overlap between online and offline spaces. One space is a supplement to the other and vice versa. Perhaps neither space can operate exclusively to achieve the desired results for civic engagement, especially with continuous advancements in technology and repeatedly obvious position of the Internet technology as a tool for communicating and organizing for community groups. New information technologies are supplemental, rather than a replacement for previous technologies. Prince (2004) and Wellman et al. (2001) found this supplement theory to be true in the communities they studied as well. An acknowledgement of online action enabled by digital technologies as supplement to offline action shows up in the interviews and journal entries. Embedded e-activists indicated in their responses that online engagements supplement the work they do offline and vice versa. These e-activists combine old and new 
media to facilitate their online and offline social action. It is obvious that the Internet and other digital sites and spaces are becoming much more relevant, if only for the simple reason that for the next 'demographic of citizens' in Western countries and elsewhere, it is the preferred space of cultural activity, communication and engagement (Volkmer, 2014). However, more needs to be done about the reach and speed, as these are important issues. As more people bypass conventional public sphere sites - often because they do not work as effectively - the Internet will only increase in significance.

\section{Civic Engagement model for Embedded e-Activists}

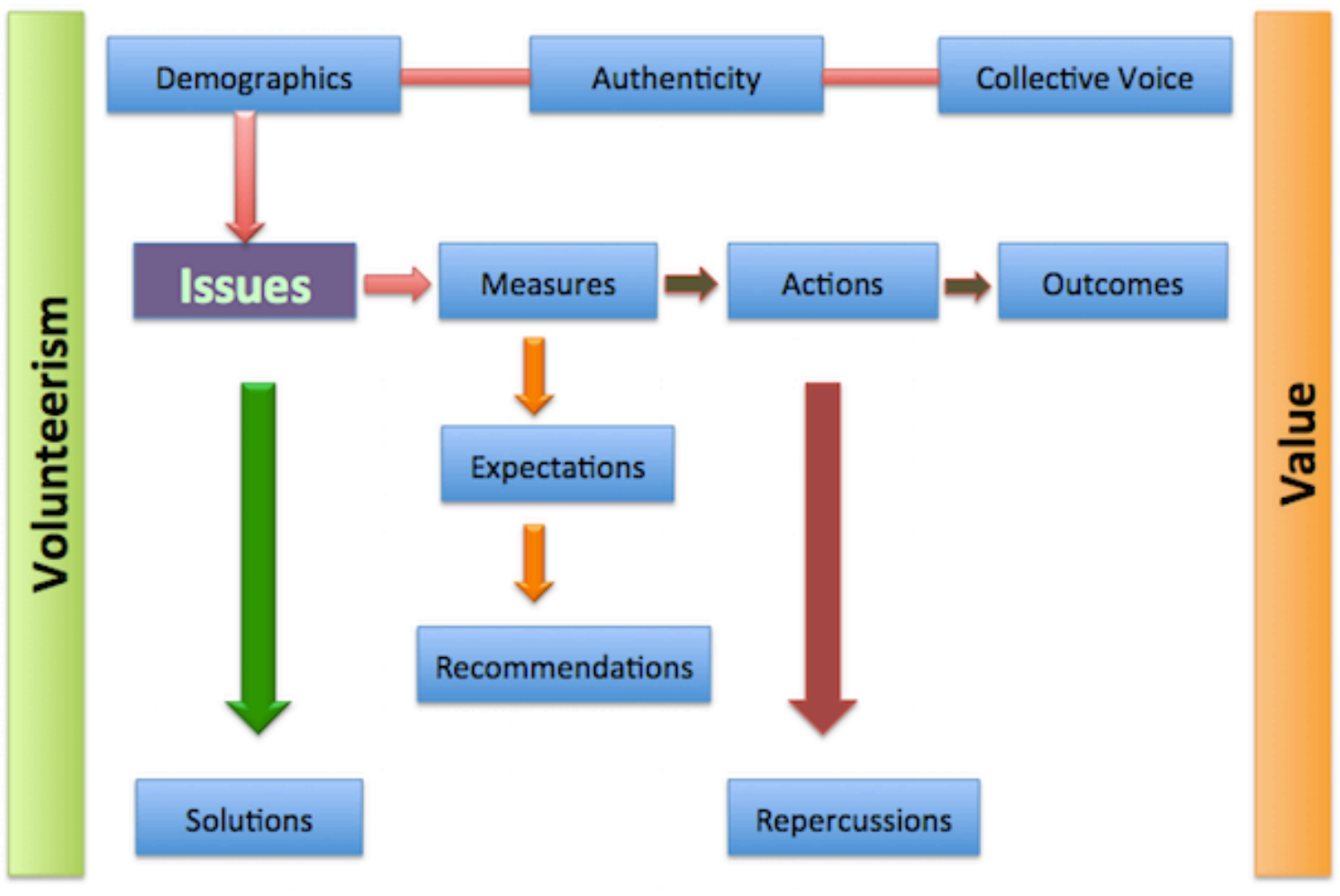

This model is specific to the typology developed for assessing the activities of embedded e-activists combining online and offline tools for civic engagement. In this model, the 
issues are central to the work of embedded e-activists; issues are the main gathering point. Most, if not all, of the processes indicated in the model are underpinned by voluntary action and service, on which these activists place a high value. It is important to identify who the people are by putting a mechanism in place for authentication in order to justify the claim of collective voice or representativeness. There are four pathways for the practices of embedded e-activists, as observed in the WorldPulse.com community. These pathways will be facilitated mostly in terms of pre- and post-activism, during daily or periodic interaction and engagement online and offline, and in e-activists' day-to-day work in local and or global communities. Each factor in the pathway can be activated at different times or simultaneously, depending on the context and the work being done. All four pathways have one common link. They all share the demographics, authenticity, collective voice and issues. No single pathway is mutually exclusive. One may find that some embedded eactivists are engaged in multiple pathways simultaneously. These four pathways are:

1) Demographics, Authenticity, Collective Voice, Issues, Solutions - with this pathway, the e-activists focus on identifying the issues and suggesting solutions.

2) Demographics, Authenticity, Collective Voice, Issues, Measures, Actions, Outcomes - this pathway is occupied by e-activists who identify the issues, suggest measures they are aware of, take actions on the solutions suggested in the first pathway or by using the existing measures, and report on the outcomes of the actions.

3) Demographics, Authenticity, Collective Voice, Issues, Measures, Expectations, Recommendations - the third pathway has e-activists who identify the issues, 
suggest measures they are aware of, discuss or outline their expectations and make recommendations.

4) Demographics, Authenticity, Collective Voice, Issues, Measures, Actions, Repercussions - this pathway shows e-activists who identify the issues and suggest measures they are aware of that can be used to address the issues; the e-activists take action and indicate their awareness of likely repercussions or they may have experienced repercussions themselves. These e-activists may also provide information about others who have experienced repercussions.

Pathways \#2 and \#3 appear to be commonly active and often in practice in the WPc community, while pathways \#1 and \#4 are also active and in practice on the platform, but not to the same degree as pathways \#2 and \#3.

Following is a meta-analysis of how the factors indicated in the civic engagement typology interact and intersect with each other:

Demographics, Authentication, and Volunteerism

Demographics is an essential feature for civic engagement and the difficulty of getting this information in online spaces should not deter researchers from working towards obtaining the information. Without knowing who is engaging, the knowledge of why they engage and what they hope to achieve may be limiting for making a case for civics, including channeling democratic reforms and policy changes. The demographics from both the 
survey and interview data show that the youth and adults are engaging on WPc.

Authentication works as an umbilical cord for demographics and volunteerism. There is a need to know that community members are indeed who they claim to be and that whatever interests they represent are made known ahead of their involvement and commitment to various issues on the site. Volunteerism is the hallmark for measuring the average WPc member. All work done on the platform, if not completed by staff, is mostly on a voluntary basis. This volunteering is problematic, but also profitable for those who through their act of selfless service get opportunities to plug into speaking engagements in and outside their local communities, connect with other members for collaboration and sometimes have opportunities to obtain fulltime jobs. Voluntary labour online is not exclusive to WPc. It is a common feature of digital media, online forums and social media platforms generally.

Issues, Actions, Measures, and Outcomes

These are critical aspects of civic engagement. What exactly are the issues? Why are these issues important? What can or should be done about them? The WPc community is very good at identifying the issues in their local communities. They go beyond the broad themes that WP staffs introduce by extending the discussions in their journal entries to outline other issues that are interconnected and interrelated to the broad themes. Examples abound in some of the journal entries on EGBV in which most journals from the Democratic Republic of Congo emphasized the need to pay attention to some women in rural communities in the DRC that were being excluded from the efforts to address issues of sex as weapon of war, as well as an international conference on issues of gender-based violence 
being held in DRC that did not have representation from members of rural communities that are directly affected by this gender-based violence.

Actions is another fairly active feature with large data sets, especially from the DACs, indicating what people have done or reports of work done by institutions to address issues or take on board the recommended measures outlined by WPc members and other people in their extended communities. Measures also appear in abundance on WPc. This feature plugs into issues, actions and outcomes although the feedback loop is missing a model for reporting the outcomes. WPc participants do not often return to the same journal entries to report the outcomes of their actions. These reports often appear in new journal entries that are hard to link or trace back to the initial journal in which the issues were raised.

Outcomes follow actions but are also very much linked to issues and measures. The nature of WPc makes it difficult to assess the outcomes members report, but digging through the pile of journal entries and comments there are indications of outcomes. Interview responses also gave insights into some of these outcomes. However, there is a need to temper expectations with reality; it would be easy to suggest that 'nothing is happening', but this would appear rather short sighted. Realistically, things are going to happen, but they may happen slowly over time. WPc community members need to be reminded of the short- and long-term goals of their social activism and then measure outcomes against these goals.

Expectations, Recommendations, Solutions, and Repercussions 
Expectations show that WPc members are very expressive in outlining their expectations, in the same way they indicate their recommendations for addressing issues. Most interview participants say they feel represented in the campaigns on WPc. This expression of representation contrasts to Bolognani's (2010) findings in her study that showed there was a lack of a unifying voice in the campaigns she assessed. Recommendations are connected to expectations, but distinct in that they are pre-emptive, active and mostly built on experiences of those putting them forward. The ability or lack thereof to make these recommendations a reality is partly the responsibility of individuals and partly that of institutions. There are reports of individuals having to take laws into their hands in some cases. An example is in the case of street sexual harassment in India. This is clearly not the best or ideal way to resolve issues, but when institutions fail, anarchy reigns. Solutions are about pre-existing issues that have been resolved and actions taken to resolve them appear in journal entries, while newly identified issues tend to be solved and reported after the completion of the DACs.

Repercussions remind one that lives are endangered in many cases in the course of civic engagement. People put themselves out there and take risks to address the issues and proffer solutions that require sacrifices on their parts. Repercussions are often negative by default. The opposite of this is benefits and such benefits would accrue in the form of policy changes and practical solutions that have come to communities as a result of actions taken by individuals and institutions. Most reports of repercussions on WPc are linked to events that have occurred prior to the launch of the DACs. This retrospective after-the-fact 
indication appeals to the format of the DACs on WPc, since the DACs require participants to reflect mostly on past experiences in writing their journal entries and comments.

Value and Collective Voice

These two factors are linked to the kind of work WPc members think the website should be doing in contrast to what it is actually doing. There is a clear indication that WPc community members place a relatively high value on their contributions to the digital action campaigns on the website; even more so, the correspondents participating in the 'Voices of Our Future' citizen journalism training program. Many of these correspondents have gone on from the campaign to become empowered women who speak out on behalf of themselves and other members in their immediate communities when they are confronted with injustice. WPc making a representation for its community members as a collective voice for women is perhaps untenable due to the multiplicity of identities of the women and the diverse nature of their interests. However, within the limits of what the website is able to do, vis-à-vis the issues that have been articulated during the campaigns and presuming that these are the issues that are of value and importance to most of the community members, such representation of communal voice will be assessed at the point of representation against the expectations of the community members.

WorldPulse.com as a Global Community 
This section reviews the idea that WPc constitutes a global community, which is quite an ambitious goal and practically unattainable, considering the diversity of women's needs and the various issues they are grappling with in their local communities. This section answers the fourth question set out in this research project:

- To what extent does the move to a virtual or digital public sphere require a reformulation of the notions of identity and identification, which is a critical issue for analyzing and understanding the roles, functions and parameters of the public sphere?

This requirement is to a relatively high extent in the case of a site like WPc that is working with mostly community members whose main currency on the website is based on their offline identities and their ability to back up the claims they make. Quite a lot is at stake if an individual is found to be fraudulent. An example external to WPc is reported in the online Global Press Journal, in which the platform had to retract a story - "for the first time in its history" - and also do an extensive investigation with detailed report of what led to this retraction (Hegranes, 2015). There is ongoing contestation of the notion of community, virtual global communities, offline local communities, and the convergence of online and offline communities resulting in more personalized connections between and among people all over the world.

WorldPulse.com as a Supportive Space 
This draws on the Habermasian criteria of discursive inclusion and equality, sincerity and ideal role taking, yet the role of WPc as a supportive space minimizes its ability to fully function as a public sphere because its autonomy is borderline and the members tend to straddle a line when it comes to their reflexive practices. As a supportive space, WPc offers a high degree of social support as DiMaggio and Hargittai recommend, but technical apparatus and variation in use make for glaring inequality.

Take, for example, Tessie's entry suggesting that women are sometimes responsible for violence because of certain personality traits that they exhibit. Arguably faintly veiled as just another submission to the EGBV campaign yet laden with many problematic assumptions, with the overriding thinking that women get what is coming to them when violence happens because, after all, they have exhibited some of the traits that Tessie listed:

It is an obvious fact that women are sometimes the catalyst to the abuses they experience and those experienced by other women. Today, I have decided to discuss some of these personality traits; so that together we can build a gender violence free environment (EGBV 1).

Tessie then goes on to list and explain each of the traits. Following is an abridged version of her list:

SELF-CENTREDNESS, DOMINEERING PERSONALITY, JEALOUS PERSONALITY, ARROGANT PERSONALITY, UN-TEACHABLE 


\author{
PERSONALITY, STURBOURN PERSONALITY, HARSH \\ PERSONALITY, DEFENSIVE PERSONALITY, SELF-PITY \\ PERSONALITY, TALKATIVE PERSONALITY, MANIPULATIVE \\ PERSONALITY, SECRETIVE PERSONALITY, NON LISTENING \\ PERSONALITY, IMPULSIVE PERSONALITY, NON-APPRECIATIVE \\ PERSONALITY, UNFORGIVING PERSONALITY, \\ UNAPPOLOGETIC PERSONALITY, TEMPERAMENTAL \\ PERSONALITY, COMPLAINING PERSONALITY (EGBV 1)
}

Out of the six comments accompanying Tessie's journal entry, only viochan's comment point out the problematic and dogmatic assumptions in the post:

I wonder about these "labels" and question whether they are influenced, if not downright created, by patriarchal thinking. Labels dismiss and invalidate women's feelings and they push women into fitting a mold that was assigned to them mostly by men. For example, most (patriarchal) societies have a standard of femininity that a woman must subscribe to. Any woman who deviates from that standard is an outcast, a trouble-maker, a bitch, etc. And why? I believe it's simply because she challenged the status quo and we all need her to fall in line and keep quiet. Because it's easier that way. So let's not be hasty in asking women to comply with what is expected of them. Or at least, let's hold men to the same standards (EGBV 1). 
This example indicates how some issues and discussions could be beneficial, while others are not on WPc in terms of how one would engage with this debate, depending on a community member's skill - specifically in this case, knowledge of the complexity of the issues of domestic and gender-based violence. The challenge of navigating the legal and security systems is another recurring issue in the discussion about violence against women. Institutional restrictions and requirements that make it difficult for women to report incidents of violence or pursue redress was a common theme in the submissions to the EGBV campaign.

WorldPulse.com Giving Women a Global and Collective Voice

It is difficult to give the women a collective voice when there is an absence of full representation of all members' interests. WPc's claim of being a global voice is an ambitious form of publicity that could undermine the practicality of what it is realistically able to do for its members. Even though women from many regions of the world are members of the platform, these women do not necessarily represent the interests of every woman in their communities. Moreover, some of these people do not participate in the campaigns. And in the likelihood that most members participate in the campaigns, there is still a likelihood of essentialism since all women do not necessarily want the same thing. The survey data indicate that WPc members are from different parts of the world. Moreover, most of the interview participants clearly articulated a diversity of subjects in terms of what other issues WPc could take on. As seen in Dahlgren (2005), in terms of representativeness, 
it is expedient to ask whose voices are heard and or represented in the debates and at the forums, as this portends a concern about a culture of essentialism, an ongoing and contested issue in feminist circles. Marx (1978) reminds us that social and class relations have an influence on our understanding of the world. By extension, the ownership of means of production as well as the power structure and dynamics in operation determines what people are enabled or constrained to do with the technology of the Internet, as well as the sites where these discussions occur (Foucault).

There are so many huge responsibilities piled on the shoulders of individual community members in the guise of volunteering as part of a global community. Very rarely is there a mention or detailed critique of the responsibilities and - quite glaring - failings of institutions to provide necessary amenities, platforms and environments for individuals to thrive and for the efforts of the individuals to be effective. For example, Nabiye Tal's journal entry alludes to these failings and simultaneously attempts to highlight these issues on the agenda of the WWTW campaign:

Also as a women's right activist, I realized that to achieve the right to communicate as a basic human right for women in Nigeria, women have to take on themselves the difficult task of gaining access to the necessary infrastructures that will enable them use the internet. This entails the provision of more infrastructures that will take care of the majority of women who reside in rural areas as well as making available the latest infrastructure for users' needs...The Nigerian government...should also 
look into concerns related to consumers' ability to pay for services, particularly in rural and poor area (WWTW 45).

WorldPulse.com as an Inclusive Platform

WPc is inclusive to the point of being exclusive. The platform is a 'safe space' for women to air their views and address issues of common interest, but it is not completely inclusive because non-conforming views do not find expression on this site. This form of inequality creates an uneven playing field in which some people's interests are privileged over others'. Perhaps inclusivity is a utopian ideal when contextualized against the perpetual presence and pervasiveness of ideology and hegemony in discourse (Foucault, 1990b). WPc as an organization has an agenda; WPc's donors have agendas and so do the members. Surface level appearance of inclusivity does not translate to absolute inclusion and representativeness. Although in the interviews participants said they feel like they are part of a global community through their interactions on WPc, especially for the participants in the global South, these participants also expressed that they feel like WPc could be doing more to specifically address needs in local communities. Most of these women are attempting to navigate the challenge of Internet access, skill for engaging in deliberation and simultaneously managing complex relationships in a virtual space.

There is also the ethical dilemma of WP management pairing DAC journal entries with commentators at the backend. In the front of the platform, this pairing aids in presenting the impression of community engagement to all community members and visitors to the WPc 
platform but backend pairing is symptomatic of attempts by WP management to make WPc appear more representative than it actually is. There are ethical issues for the volunteer commentators, who are somewhat compelled to comment on journal entries even when they do not want to, but also have to make certain and or specific kinds of comments because their commenting activities are 'policed' by WP management; an issue that will be of concern to Foucault.

WorldPulse.com as an Accessible Platform

DiMaggio and Hargittai (2001) remind us to look beyond technological accessibility, but for some communities access is still an issue and this compounds the inequality because even though the people now have access, the access is either not guaranteed, limited or threatened such that the other forms of inequalities - especially looking at soft skills becomes a secondary issue and sometimes the affected people gloss over this issue, since they are first and foremost just so thankful that they can at least gain access to the technology. The digital divide is still a dilemma in many communities. As attention shifts to digital inequality, the digital divide must be queried with as much rigor as is afforded the challenges of digital inequalities. Accessibility also brings up issues of different levels of physical abilities of existing and potential members. Accessibility is not just about technological access but how to also represent the interests of and get participation from people who may be hard-of-hearing, blind or have limited use of their limbs.

WorldPulse.com Influencing Policies in Local and Global Communities 
WPc says policy influence happens frequently at the broader level and in large global contexts, but most interview participants do not see evidence of such influences at the local level. There is a need to find a way of measuring such policy influence in local communities to be able to authenticate the claims and perhaps establish a feedback loop that emphasizes the indicators of such policy influence at both micro and macro levels. For instance, the EGBV campaign is cited as having an influence in the appointment of a U.S. envoy to the Democratic Republic of Congo (WP staff 1). While this specific appointment in the Democratic Republic of Congo continues to be cited by WPc as one of its accomplishments several years after it has occurred, there is no commensurate citing of other policy changes that are happening in other communities locally or at any collective level globally.

WorldPulse.com, Identity and the Digitalized Public Sphere

One of the most significant points that has emerged from the analysis of the WorldPulse.com site and its activities is that the criteria for public sphere activity identified by Habermas and neo-Habermasians such as Dahlberg (for example, authenticity and sincerity), are abstractions or ideals that do not easily tie in or equate with everyday experiences, activities and practices. This is not a new insight: but what is important in my analysis of WPc is the understanding that these abstractions, while not being 'real' or genuine 'things', are performatives that are (usually) still required in public sphere activity. What this leads to is the further understanding that the public sphere is not a stable, 
continuous and unmediated reality; rather it is something that is 'culturally evaluated and recognized'. What we might call efficacious or felicitous public sphere activity and involvement requires a set of performatives that are commensurate with a set of criteria that may vary from one culture to another, and can be read and recognized differently from one culture to another.

The transition from public sphere activity 'in the flesh' to digital 'presences' and traces, means that there is a need to revise or at least clarify or qualify Habermasian criteria to some extent, and to explain that the question of performatives, and the recognition of those performatives, has moved on quite considerably from the time of Habermas. By way of extension, an argument can be made that the move to the digital public sphere undermines the prior notions of identity, and consequently requires a reappraisal of concepts and criteria such as authenticity, empathy and sincerity. It is virtually impossible to guarantee identity - and authenticity - in the digital public sphere. The issue that comes into play, from this perspective, is the extent to which virtual identity is consistent and commensurate with regard to performatives both of offline identity and its identity categories (male, vegetarian, feminist, South African, mother, journalist), and the criteria that define felicitous public sphere activity (one must perform empathy, one must be seen to be sincere).

A further issue is the extent to which the contexts of the site and space where the public sphere activity occurs - as with WPc - has its own requirements that inform and delimit what is recognized as acceptable. We have seen that WPc disciplines subjects in terms of 
how other participants are addressed and the tenor of interpersonal relations; and it also delimits forms of communicative action and activity. It has its own culture, values and imperatives, and these are discursively marked and clearly enforced, one way or anotherimplicitly rather than explicitly. Moreover participants are aware of, and adhere to, these requirements most of the time.

Participation in the Digital Public Sphere

Another issue that has arisen out of the research on WorldPulse.com concerns the motivation behind people participating in the digital public sphere, and the extent to which those forms of motivation are commensurate with Habermas' largely abstracted and idealized prescriptions regarding the tenor of political communication. As we pointed out in earlier chapters, for Habermas public sphere activity is necessarily predicated on rationality and reflective consideration. This is not necessarily antithetical to the culture that informs both communication and participation on WPc: the strong discursive commitment to the other and otherness is compatible with the notion of rationality and reflexivity; and conversely reflexivity is an important theoretical component of political movements such as feminism, anti-colonialism and socio-cultural activities that seek, in Bourdieu's terms, to universalize access to the universal (Bourdieu, 1999).

On WPc, however, the culture of political engagement does not sit easily with discourses of disinterestedness; and the abstraction of socio-cultural issues and relations as being 'without regard to' the realities of power and political oppression would be understood and treated as 
a form of symbolic violence. In this regard Foucault's work, and in particular his accounts of discourse and power, and Bourdieu's theorizing of the relation between language and symbolic violence (Bourdieu, 2005) provide a much more relevant and useful set of resources for understanding how and why disinterestedness and discourses of abstracted rationality are not part of the culture of WPc.

\section{Conclusion}

WorldPulse.com clearly does have the capacity to function as a micro public sphere for marginalized groups. One of the ways it does this is by combining online and offline tools for the civic engagement activities and events that its members do. The most common tools for engaging the platform's members in civics are the digital action campaigns and the 'Voices of Our Future' citizen journalism training programs. The platform is not completely autonomous but as I have discussed in earlier chapters, this is not a necessity for it to function as a public sphere. The need for community members to experience inclusion and equality challenges the rhetoric and ability of the WPc forum to fully engage in criticalrational debates. Through its use of technology for convening meetings among widely dispersed people in different parts of the world, WPc offers a global public sphere for those who have the interest and are willing to invest the time and other resources in engaging in the issues discussed on the website. Some of the constraints on the website include the reality of contestable membership and active participation in online space, which requires a level of spectacle and adaptability that do not necessarily fit Habermas' model. WorldPulse.com makes representations on behalf of its members online and at offline 
forums, but it cannot necessarily represent the interests of all women, as this is an impossible and impractical essentialist ideal. As much as the website tries to be inclusive, it is clear that the members are not on an equal playing field with each other, and even more so with the owners of the website.

The digital divide is still a pressing issue with regard to the viability of Internet technology as a basis for civic engagement. This issue is relevant to both the Northern and Southern Hemisphere, but it is perhaps especially relevant during periods of civic unrests in authoritarian regimes. Despite the fact that most WPc community members own multiple devices with which they can access the Internet, the experience of access varies significantly among the interview respondents. This divide doubly disadvantages women who do not have the other necessary capacities like technical knowledge and language skill or social support. The variation in use of the devices is linked to the kinds of Internet connectivity to which the respondents have access as well as the respondents' concerns (or lack thereof) about privacy, which is strongly linked to the surveillance practices of governments and other organizations. Although WPc community members have an overwhelming amount of autonomy of use due to them owning multiple devices, the availability of social support determines whether or not the members can take full advantage of the autonomy of use, most especially for civic engagement. Autonomy of use and availability of social support are intersecting and overlapping concepts that can either limit or increase community members' sense of experiences of WPc as a global space for people with similar interests. 
Embedded e-activists participate in and experience civic engagement in multiple and diverse ways, depending on their locations offline. They simultaneously connect their experiences with other social activities outside of their immediate locale through the work they do in online forums such as WPc. These social and political activists are often referred to as citizen journalists; however, they are re-envisioned as embedded e-activists in this research project based on the context of the extensive work they do and the investments of diverse resources they make, while engaging in civics both online and offline. Embedded eactivists' experiences can sometimes appear to be happening in isolation, to the detriment of both community members and management of WPc, especially in the context of the latter's attempt to influence policies in local and global communities. Developing and disseminating mechanisms for assessing and reporting on various achievements that can be linked to the activism on the platform will aid in further ensuring that the individual gains being made by community members can be properly cited.

Interview participants talked about the need for WPc to consider country-specific initiatives, as opposed to trying to do global programs whose impacts in local communities are harder to measure. WPc as a global community relies on the engagement and experiences of its members located in various parts of the world. Authenticity is a major currency for members engaging in the forum. The platform offers a supportive space to its community of social activists to the degree that it is able to both online and offline. The forum needs to avoid essentializing discourses that position it as capable of representing all women's interests, especially given that women are not homogeneous in their needs and experiences. WPc's attempts to be an inclusive platform sometimes make it difficult for it to fully 
engage with difficult issues that clearly come with a range of diverse opinions, some of which may not be commonly supported by the community members, and hence result in exclusionary practices. The work carried out by this dissertation suggests that WPc does function as a micro public sphere, but it needs to be aware of its own limitations as a virtual space within a larger and broader network of such spaces that are available online for civic engagement. 


\section{Conclusion}

The questions of to what extent and how effectively the WorldPulse.com forum functions as a public sphere site are part of a wider set of questions that have arisen out of the transformation of communication activities, practices and networks by and through digital technology. My goal was to produce a study that was reflexive, collaborative, and which produced research that made a significant contribution to the wider discussion - being carried on across various academic fields and disciplines - of the relation between public sphere activity and contemporary communication technologies. As well as these more scholarly aims and objectives, I would like to think that this study might also prove to be of benefit to the virtual community I was studying, as well as to those groups of women in both local and global communities who use new media technologies to engage with and address issues of common interest.

The Community on WorldPulse.com

WorldPulse.com claims to be intent on creating a space for its members to gather and experience a sense of community; share information; discuss a broad range of issues; identify problems, both general and specific, that afflict women globally; exchange experiences; provide accounts of different forms of physical and symbolic violence that are directed against women, and are endemic to the communities in which they live; offer sympathy and support; suggest solutions; and contribute critiques of the ways in which the power differentials which facilitate acts and cultures of violence are reinforced and 
naturalized. However, it became equally clear that the website culture had its limitations in terms of functioning as a public sphere site or set of spaces, particularly if the criteria applied to this function was derived from or strongly influenced by the theories and work of Habermas. These ranged from conditions and contexts which militated against allowing the full diversity of group representation, to the difficulty of ensuring that the discursive activity had any tangible results, apart from increasing the level of awareness of the members. However, perhaps the most significant factor was the culture of WorldPulse.com itself: quite understandably, the site is at pains to provide a space where participants who have been the subject of various forms of violence can receive sympathy, empathy, advice and support. However to some extent this strong imperative works against the possibility of what we might refer to as more robust or critical discussion. This tended to limit the effectiveness of WorldPulse.com as a site for debate.

Further, the messages of the platform are bound to resonate with some groups more than others. Evidence of liberal, left-leaning, socialist 'thinking' suggests that women with conservative and differing opinions may not be represented equally on the platform. There is little evidence of self-critique, nor of any awareness of the power imbalance among participants, and between participants and owners of the site. Hence while it appears that it is hard for anyone not to commit to the ideals of the public sphere, the proponents of these ideals need not necessarily actualize or reproduce them in their communicative practices.

At the same time this dissertation came to the conclusion, based on its study of WPc, along with its activities, communication practices and overriding culture, that the WPc culture 
could only be characterized as a limitation if the set of criteria that Habermas and his followers prescribed for public sphere activity was in fact viable, credible and necessary to an evaluation of the public sphere. It became clear that while there was a considered gulf between Habermas' prescriptions and WPc practices, it did not necessarily follow that WPc did not function as a viable public sphere space. On the contrary, it became apparent that WPc did in fact facilitate, encourage and engender civic engagement and discussion, across a global audience. Consequently the focus of my research, or at least of my theoretical evaluation of the question of WPc as a public sphere space, turned to the extent of the usefulness and viability of Habermas' prescriptions about the public sphere. To sum up: the focus of this dissertation moved from an evaluation of the fit between Habermas' prescriptions and possible public sphere sites, to a consideration of, firstly, how the identity of the public sphere was plural rather than singular; secondly, that sites that performed a public sphere function would necessarily be characterized by specific cultural, discursive and generic features; and thirdly and consequent of the first two propositions, that a set of abstract prescriptions based on the notion of a singular public sphere character and identity in fact performed an act of (symbolic) violence upon the plurality of public sphere sites.

The Public Sphere on WorldPulse.com

This dissertation both provided a critique of and attempted to develop and expand upon the Habermasian conceptualization of the public sphere and public sphere activity. The nature of WPc as a technology and a community of people from diverse backgrounds largely shape the manifestation, or lack thereof, of the Habermasian public sphere criteria. 
Autonomy of community members manifests in varying degrees, while that of the platform is contested. Exchange and critique is scarce and perhaps not a practical expectation for such a platform as WPc. It can be argued that the culture neither facilitates robust debates nor the articulation of differing positions and perspectives.

Reflexivity in Habermas' terms is scarce; it often manifests as expressions of gratitude for the willingness of people to share personal stories. However as I have pointed out in previous chapters, the WPc site and its communication practices are clearly informed by, and built upon, a sense of reflexivity tied to and derived from the discursive value accorded to, and the respect required for, otherness. Ideal role taking is commonplace on WPc and the site is well suited to demonstrate this criterion. Sincerity is an essential marker of civic engagement and it is common on WPc, but the extent to which sincerity can be identified and validated is difficult to assess. The lack of continuity between digital identity and the position of participants on the issues they discuss poses a challenge to the assessment of this criterion, except in terms of levels of discursive performativity and consistency.

An assessment of the data for discursive inclusion and equality indicates that WPc attempts to be an inclusive space. Such level of inclusivity is uncommon in virtual spaces generally. The requirements of sincerity, reflexivity and ideal role taking compete with the need for critical-rational debate. Inequality in technical apparatus, autonomy of use, skill, and availability of social support were all demonstrated in the data. There are somewhat similar challenges with digital apparatus in both the Northern and Southern Hemispheres. Autonomy of use of digital devices is commonplace among WPc community members. 
However, the language barrier and a lack of knowledge of the complexity of issues being discussed contribute to the level of inequality on the site.

While there is very limited report of policy changes that could be traced directly to campaigns on WPc, in most cases the aims and objectives of campaigns, and more generally of the site as a communal space, are tied in with raising awareness, providing support and feedback, and functioning as an alternative space where disadvantaged groups can hold discussions and debates. These aims and objectives do not necessarily translate into immediate policy or political changes. However, short- and long-term goals of social activism need to be clearly articulated at the beginning of each campaign so that the eactivists can measure outcomes against these goals realistically.

Authenticity of community members and the issues they identify are managed through interactions between WPc community members and management. E-activists combine old and new media to manage this task and by extension facilitate their online and offline social action. However, it is difficult to give women a collective voice since it is impossible to represent the interests of the diversity of people engaging on the platform. WPc is inclusive almost to the point of being exclusive; the appearance of inclusivity does not always translate into inclusiveness and representativeness.

Problems and Difficulties 
Assessing publicly accessible archived journals and comments for this research posed some problems in terms of ethical consideration for the identity of the writers. All the journals used in this research are publicly available and accessible on the WorldPulse.com website without needing to log into the private space; however, some of the information the women shared in their journals was quite personal. I have retained the identities of the journal and comment writers as they appear on the publicly accessible part of WPc, but all identifying information in the online web survey and email interview responses have been removed.

There were fluctuations in the pre-identified boundaries of the field of virtual ethnography for this study. To some extent, I was conducting an auto-ethnographic study: I was a member of the website and also positioned as a participant-observer. The ongoing relationship between online and offline personas was challenging as both spaces act on each other. It was sometimes difficult to not look at other sections of the website in detail when working in the distinct sections that were already mapped out as constituting the field of research. This difficulty posed a challenge because there is material in other sections of the website that potentially holds information that could be useful for what I was doing in the delineated field. By extension, the activities taking place offline that could be traced back to the WorldPulse.com site also created a level of difficulty for confining myself to the pre-determined research field.

Reflecting on the potential (or not) to generalize the results of this research, I would say that based mainly on sample size - this is one case study of one specific site, although the site offered an extensive amount of archival journal writings that were complemented with 
survey and interview data - this study is a small one and its findings should not be generalized to other online forums.

Future Research

There are opportunities to conduct future research that expand on some of the findings of this thesis. At a specific level, further research could focus more closely on various programs, such as the 'Voices of Our Future' citizen journalism training. It could also analyze the reports and publications that are produced after the digital action campaigns; the offline conferences World Pulse organizes; and the activities of the local community organizations that are linked to WorldPulse.com.

Another opportunity for future research includes an extended study of online forums that use virtual and physical ethnography to assess the work of community members online in relation to their physical, real-life work offline. Such a study would benefit from the application of the civic engagement typology. There could also be an institutional ethnographic study of an online forum that includes all internal and external stakeholders, such as forum members, site moderators, site owners, sponsors, funders, technology providers; and some of the institutions whose work affect the forum, including governments, schools and religious organizations. Such a study would benefit from the use of DiMaggio and Hargittai's model for analysis with a specific focus on how digital inequality is institutionalized. 
At a more general level, further research could consider the extent to which the Habermasian version of the public sphere, and the criteria associated with it, are compatible with regard to the communicative practices, genres, forms of interaction and technologies of digital culture. One of the problems - one of many - in this regard is the question of identity. When Habermas describes, defines and characterizes the public sphere, he tends to take the notion of identity for granted; that is to say, he more or less presumes a stable, selfaware, rational, reasonable and consistent (and predominantly male, Western and educated) identity. In effect the Habermasian public sphere is a strongly gendered space that also privileges specific dispositions, literacies and forms of capital. However, perhaps an equally important consideration is the extent to which the notion of an authentic, consistent and knowable identity is largely incommensurate with the communicative practices and contexts of online and digital communities. This last issue was a significant point in, and problem for, my research. However it was not the sole focus of my research, nor did the scope of my research allow it to be dealt with at the level of complexity and focus that it required. 
Bibliography

Abraham, J. (2010). Veiling and the Production of Gender and Space in a Town in North India: a Critique of Public/Private Dichotomy. Indian Journal of Gender Studies, $17(2), 191-222$.

Adorno, T., \& Horkheimer, M. (1972). The Culture Industry: Enlightenment as Mass Deception. In J. Cumming (Trans.), Dialectics of Entitlement. New York: Herder and Herder.

Albrecht, S. (2006). Whose Voice is Heard in Online Deliberation? A Study of Participation and Representation in Political Debates on the Internet. Information, Communication, and Society, 9(1), 62-82.

Alzouma, G. (2013). Dimensions of the Mobile Divide in Niger. In Ragnedda, M. and Muschert, G.W. (Eds.), The Digital Divide (pp. 295-306). London: Routledge.

Babbie, E., \& Benaquisto, L. (2002). Fundamentals of Social Research. Canada: Thomas Nelson.

Bakardjieva, M. (2011). Reconfiguring the Mediapolis: New Media and Civic Agency. New Media \& Society, 14(1), 63-79. http://doi.org/10.1177/1461444811410398.

Bakhtin, M. (1986). Speech Genres \& Other Essays. Austin: University of Texas Press. Banner, D. J., \& Albarran, J. W. (2009). Computer-Assisted Qualitative Data Analysis Software: A Review by Davina J. Banner and John W. Albarran - Research Rounds. Canadian Journal of Cardiovascular Nursing, 19(3).

Becker, H. S. (2004). Whose Side Are We On? In W. K. Carol (Ed.), Critical Strategies for Social Research (pp. 23-28). Toronto: Canadian Scholars' Press Inc. 
Bethlehem, J., \& Biffignandi, S. (2011). Handbook of Web Surveys: Best Practices to Create and Implement Highly Effective Web Surveys. New Jersey: John Wiley \& Sons.

Birchall, C. (2016). Digital networks of political action. Retrieved from http://2plqyp1e0nbi44cllfr7pbor.wpengine.netdnacdn.com/files/2013/01/OnlineNetworksOfAction-Birchall-FinalReport.pdf.

Blumler, J. G., \& Gurevitch, M. (2001). The New Media and Our Political Communication Discontents: Democratizing Cyberspace. Information, Communication \& Society, 4(1), 9-10.

Boeder, P. (2005). Habermas' Heritage: the Future of the Public Sphere in the Network Society. First Monday, 10(9), 1-11.

Bolognani, M. (2010). Virtual Protest with Tangible effects? Some Observations on the Media Strategies of the 2007 Pakistani anti-Emergency Movement. Contemporary South Asia, 18(4), 401-412. http://doi.org/10.1080/09584935.2010.526198.

Born, G. (2013). Chapter 7. Mediating the Public Sphere: Digitization, Pluralism, and Communicative Democracy. In Emden and Midgley (Eds.), Beyond Habermas: Democracy, Knowledge, and the Public Sphere (pp. 119-146). U.S.: Berghahn Books.

Bourdieu, P. (1986). The Forms of Capital. Retrieved from https://www.marxists.org/reference/subject/philosophy/works/fr/bourdieu-formscapital.htm.

Bourdieu, P. (1989). Social Space and Symbolic Power. Sociological Theory, 7(1), 14-25. Bourdieu, P. (1991). Outline of a Theory of Practice. Cambridge: Cambridge University Press. 
Bourdieu, P. (1996). The State Nobility. Oxford: Polity Press.

Bourdieu, P. (1999). The Weight of the World. Cambridge: Polity Press.

Bourdieu, P. (2000). Pascalian Meditations. Cambridge: Polity Press.

Bourdieu, P. (2005). Language and Symbolic Power. Cambridge: Polity Press.

Bradley, C., \& Shroeder, R. D. (2009). Because It's Free Poker! A Qualitative Analysis of

Free Texas Hold'Em Poker Tournaments. Sociological Spectrum, 29(3), 401-430.

Brenner, S. (2011). Private Moralities in the Public sphere: Democratization, Islam, and Gender in Indonesia. American Anthropologist, 113(3), 478-490.

Bryman, A., Teevan, J. J., \& Bell, E. (2009). Content Analysis. In Social Research Methods (pp. 295-300). Don Mills, Ontario: Oxford University Press.

Burns, E. (2010). Developing Email Interview Practices in Qualitative Research. Sociological Research Online, 15(4), 1-12.

Butler, J. (2004). Undoing Gender. London: Routledge.

Butler, J. (2005). Giving An Account of Oneself. New York: Fordham University Press.

Butler, J. (2006). Precarious Life. New York: Verso.

Cameron, J. (1998). The Practice of Politics: Transforming Subjectivities in the Domestic Domain and the Public Sphere. Australian Geographer, 29(3), 293-307.

Chen, G. M. (2011). Tweet this: a Uses and Gratifications Perspective on How Active Twitter Use Gratifies a Need to Connect with Others. Computers in Human Behavior, 27(2), 755-762.

Chipchase, J. (2011). Is Internet Access a Human Right? Retrieved from http://globalpublicsquare.blogs.cnn.com/2011/07/14/is-Internet-access-a-humanright/. 
Dahlberg, L. (2001). Extending the Public Sphere through Cyberspace: The Case of Minnesota E-Democracy. First Monday, 6(3), 1-15.

Dahlberg, L. (2005). The Habermasian Public Sphere: Taking Difference Seriously? Theory and Society, 34(2), 111-136.

Dahlgren, P. (2005). The Internet, Public Spheres, and Political Communication: Dispersion and Deliberation. Political Communication, 22, 147-162.

Dahlgren, P. (2012). Public Intellectuals, Online Media, and Public Spheres: Current Realignments. International Journal of Politics, Culture \& Society, 25, 95-110. Dahlgren, P. (2014). Political Participation via the Web: Structural and Subjective Contingencies. Interactions: Studies in Communication \& Culture, 5(3), 255-269.

Dean, J. (2003). Why the Internet is not a Public Sphere. Constellations, 10(1), 95-112. Debord, G. (2006). The Society of the Spectacle. New York: Zone Books.

DeVault, M., \& McCoy, L. (2001). Institutional Ethnography: Using Interviews to Investigate Ruling Relations. In W. K. Carol (Ed.), Critical Strategies for Social Research (pp. 191-205). Toronto: Canadian Scholars' Press Inc.

Dillman, D. A., Smyth, J. D., \& Christian, L. M. (2008). Internet, Mail and Mixed Mode Surveys: The Tailored Design Method. New Jersey: John Wiley \& Sons.

DiMaggio, P., \& Hargittai, E. (2001). From the "Digital Divide" to "Digital Inequality": Studying Internet Use as Penetration Increases. In Center for the Arts and Cultural Policy Studies (Vol. 15, pp. 1-23). Princeton University.

Dyer-Witheford, N. (2002). E-Capital and the Many Headed Hydra. In E. Elmer (Ed.), Critical Perspectives on the Internet (pp. 129-63). Lanham, MD: Rowmand and Littlefield. 
Education for All 2000-2015: Achievements and Challenges. (n.d.). Retrieved from http://en.unesco.org/gem-report/report/2015/education-all-2000-2015achievements-and-challenges\#region-report-gallery.

Edwards, L. (2015). Interrogating the Complexities of Digital Communication for Young People Engaged in Social Action. Working Papers of the Communities \& Cultures Network+, Vol. 6 October.

Emden, C.J. \& Midgley, D. (2013). (Eds.) Beyond Habermas: Democracy, Knowledge, and the Public Sphere. U.S.: Berghan Books.

Etling, B., Kelly, J., Faris, R., \& Palfrey, J. (2010). Mapping the Arabic Blogosphere: Politics and Dissent Online. New Media \& Society, 12(8), 1225-1243. http://doi.org/10.1177/1461444810385096.

Faris, D.M. (2013). Digitally Divided We stand: the Contribution of Digital Media to the Arab Spring. In Ragnedda, M. and Muschert, G.W. (Eds.), The Digital Divide (pp. 207-219). London: Routledge.

Flyvbjerg, B. (2004). Five Misunderstandings About Case-Study Research. In C. Seale, G. Gobo, J. F. Gubrium, \& D. Silverman (Eds.), Qualitative Research Practice (pp. 420-434). Thousand Oaks: Sage.

Foteinou, G. (2010). E-Exclusion and the Gender Digital Divide. Computers and Society, 40(2), 50-61.

Foucault, M. (1972). The Archeology of Knowledge. New York: Pantheon.

Foucault, M. (1990a). Method. In R. Hurley (Trans.), The History of Sexuality, Vol. 1: an Introduction (pp. 92-102). New York: Vintage.

Foucault, M. (1990b). The Incitement to Discourse. In R. Hurley (Trans.), The History of 
Sexuality, Vol. 1: an Introduction (pp. 17-35). New York: Vintage.

Foucault, M. (2000). Truth and Power. In J. D. Faubion (Ed.), Power (pp. 116-133). New York: New Press.

Foucault, M. (2007). Security, Territory, Population: Lectures at the College de France 1977-1978. (M. Senellart, Ed.). New York: Palgrave.

Foucault, M., \& Faubion, J. D. (2000). Interview with Michel Foucault. In Power (pp. 288293). New York: New Press.

Fraser, N. (1992). Rethinking the Public Sphere: A Contribution to the Critique of Actually Existing Democracy. In C. Calhoun (Ed.), Habermas and the Public Sphere (pp. 109-142). Cambridge: MIT Press.

Fraser, N. (2007). Transnationalizing the Public Sphere: on the Legitimacy and Efficacy of Public Opinion in a Post-Westphalian World. Theory, Culture \& Society, 24(4), 730.

Frow, J. (2015). Genre: the New Critical Idiom. Routledge.

Gamble, A. (2004). Public Intellectuals and the Public Domain. New Formations, 41-53.

Garcia, A.C., Standlee, A.I., Bechkoff, J., Yan, C. (2009). Ethnographic Approaches to the Internet and Computer-Mediated Communication. Journal of Contemporary Ethnography, 38(1), 52-84. http://citeseerx.ist.psu.edu/viewdoc/download?doi=10.1.1.1001.8540\&rep=rep1\&ty pe $=$ pdf.

Giwa, J. (2015). The Comment is the News. Retrieved from http://www.artempt.com/thecomment-is-the-news/.

Gordon, J. (2002). The Mobile Phone: An Artefact of Popular Culture and a Tool of the 
Public Sphere. Convergence: The International Journal of Research into New Media Technologies, 8(3), 15-26. http://doi.org/10.1177/135485650200800303

Graham, S. L. (2003). Making the Private Public: a Brazilian Perspective. Journal of Women's History, 15(1), 28-42.

Gring-Pemble, L. M. (1998). Writing Themselves into Consciousness: Creating a Rhetorical Bridge Between the Public and Private Spheres. Quarterly Journal of Speech, 84, 41-61.

Gulyas, A. (2015). Social Media and Community Volunteering. Retrieved from http://2plqyp1e0nbi44cllfr7pbor.wpengine.netdna-cdn.com/files/2014/08/Socialmedia-and-community-volunteering-Final-report.pdf.

Habermas, J. (1974). The Public Sphere: an Encyclopedia Article. New German Critique, 3, $49-55$.

Habermas, J. \& McCarthy, T. (1981). The Theory of Communicative Action - Volume 1. Reason and the Rationalization of Society. Boston: Beacon Press.

Habermas, J. (1990). Discourse Ethics: Notes on Philosophical Justification. In Moral Consciousness and Communicative Action (pp.43-115). Cambridge: MIT Press.

Hall, S. (1993). Encoding/Decoding. In S. During (Ed.), The Cultural Studies Reader (pp. 90-103). London: Routledge.

Hampton, K.N., Goulet, L.S., \& Albanesius, G. (2015). Change in the Social Life of Urban Public Spaces: the Rise of Mobile Phones and Women, and the Decline of Aloneness over 30 years. Urban Studies, 52(8), 1489-1504.

Harte, D. (2014). New Knowledge Networks in Communities - the Role of 'Hyperlocal' Media Operations in Facilitating Everyday Digital Participation. Retrieved from 
http://2plqyp1e0nbi44cllfr7pbor.wpengine.netdna-cdn.com/files/2013/01/NewKnowledge-Networks-in-communities-HARTE.pdf.

Harvey, D. (2004). The Principles of Dialectics. In W. K. Carol (Ed.), Critical Strategies for Social Research (pp. 125-132). Toronto: Canadian Scholars' Press Inc.

Hatuka, T. \& Toch, E. (2016). The Emergence of Portable Private-Personal Territory: Smartphones, Social Conduct and Public Spaces. Urban Studies, 53(10), 2192-2208.

Hegranes, C. (2015, November 5). Inside an Error: Sources Lied, But We Should Have Checked. Retrieved from http://globalpressinstitute.org/blog/inside-an-errorsources-lied-but-we-should-have-checked/

Hine, C. (2000). Virtual Ethnography. London: Sage Publications Limited.

hooks, b. (1990). Culture to Culture: Ethnography and Cultural Studies as Critical Intervention. In Yearning: Race, Gender, and Cultural Politics (pp. 121-133). Boston: South End Press.

Houghton, T. J. (2012). "Land of the Long Black Cloud": Copyright, Globalization and Viral Counterpublicity in Aotearoa New Zealand. Journal of Broadcasting and Electronic Media, 56(4), 511-528.

Internet Corporation for Assigned Names and Numbers. (2012, February 20). Retrieved from https://www.icann.org.

Isekeije, J. (2012, April 8). Depopulating the Political Sidelines: CBC News Online Forum and Public Spheres in Canada. Brock University, Ontario. Retrieved from http://dr.library.brocku.ca/handle/10464/4257.

Janack, J. A. (2006). Mediated Citizenship and Digital Discipline: a Rhetoric of Control in a Campaign Blog. Social Semiotics, 16(2), 283-301. 
Janesick, V. J. (1999). A Journal About Journal Writing as a Qualitative Research Technique: History, Issues, and Reflections. Qualitative Inquiry, 5, 505-524.

Jensen, J. L. (2003). Public Spheres on the Internet: Anarchic or Government-Sponsored A Comparison. Scandinavian Political Studies, 26(4), 349-374.

Jones, B. G. (2007). Explaining Global Poverty: a Realist Critique of the Orthodox Approach. In W. K. Carol (Ed.), Critical Strategies for Social Research. Toronto: Canadian Scholars' Press Inc.

Kirby, S. L., Greaves, L., \& Reid, C. (2006). Method One: Surveys. In Experience Research Social Change: Methods Beyond the Mainstream (pp. 127-146). Toronto, Ontario: Broadview Press.

Kravets, D. (2011). U.N. Report Declares Internet Access a Human Right. Retrieved from http://www.wired.com/threatlevel/2011/06/internet-a-human-right/.

Krippendorff, K. (2004). Content Analysis: an Introduction to its Methodology. Thousand Oaks, California: Sage.

Kuhn, T. (1970). The Structure of Scientific Revolutions. Chicago: University of Chicago Press.

Larsen, J. (2013, August 16). The Global Girl Tipping Point. Retrieved from http://www.feminist.com/resources/womensglobalvoices4.html.

Lefort, C. (1986). The Political Forms of Modern Society. Cambridge, Massachusetts: MIT Press.

Levinas, E., \& Nemo, P. (1985). Ethics and Infinity: Conversations with Philippe Nemo. Michigan: University Press.

Liff, S., \& Shepherd, A. (2003), ‘An Evolving Gender Digital Divide?', OII Issue Brief No. 
2, Oxford: Oxford Internet Institute. Retrieved from www.ox.oii.ac.uk/resources. Lippman, W. (1925). The Phantom Public. New York: Harcourt, Brace.

Liptak, K. (2012, November 8). Report Shows Turnout Lower than 2008 and 2004. Retrieved from http://politicalticker.blogs.cnn.com/2012/11/08/report-showsturnout-lower-than-2008-and-2004/.

Lockyer, S. (2006). Heard the One About... Applying Mixed Methods in Humour Research? International Journal of Social Research Methodology, 9(1), 41-59.

Lunt, P., \& Livingstone, S. (2013). Media Studies' Fascination with the Concept of the Public Sphere: Critical Reflections and Emerging Debates. Media, Culture \& Society, 35(1), 87-96.

Markell, P. (1997). Contesting Consensus: Rereading Habermas on the Public Sphere. Constellations, 3(3), 377-400.

Marx, K. (1978). Ruling Class and Ruling Ideas. In R. C. Tucker (Ed.), The Marx and Engels Reader (pp. 172-175). New York: W.W. Norton.

Marx, K., \& Tucker, R. C. (1978). Introduction to a Critique of Political Economy. In The Marx and Engels Reader (pp. 222-250). New York: W.W. Norton.

Mau, A. (2015, March 7). The Curse of \#casualsexism. Retrieved March 17, 2015, from http://www.stuff.co.nz/life-style/life/67045518/the-curse-of-casualsexism.

McCarthy, J. R., Holland, J., \& Gillies, V. (2003). Multiple Perspectives on the "Family" Lives of Young People: Methodological and Theoretical Issues in Case Study Research. International Journal of Social Research Methodology, 6(1), 1-23.

McKenna, G. \& Edwards, L. (2016). Giving Social Action a Voice: reframing Communication as Social Action. Retrieved from 
http://2plqyp1e0nbi44cllfr7pbor.wpengine.netdna-cdn.com/files/2013/01/GivingSocial-Action-a-Voice-FINAL.pdf.

Meho, L. I. (2006). Email Interviewing in Qualitative Research: a Methodological Discussion. Journal of the American Society for Information Science and Technology, 57(10), 1284-1295.

Meinrath, S.D., Losey, J., \& Lennett, B. (2013). Afterword: Internet Freedom, Nuanced Digital Divides, and the Internet Craftsman. In Ragnedda, M. and Muschert, G.W. (Eds.), The Digital Divide (pp. 307-313). London: Routledge.

Moe, H. (2008). Dissemination and Dialogue in the Public Sphere: a Case for Public Service Media Online. Media, Culture \& Society, 30(3), 319-333.

Murthy, D. (2008). Digital Ethnography: an Examination of the Use of New Technologies for Social Research. Sociology, 42(5), 837-855.

Murthy, D. (2011). Emergent Digital Ethnographic Methods for Social Research. In S. N. Hesse-Biber (Ed.), The Handbook of Emergent Technologies in Social Research (pp. 158-179). New York: Oxford University Press, Inc.

Napoli, P. M. (2010). Audience Evolution: New Technologies and the Transformation of Media Audiences. New York: Columbia University Press.

O’Reilly, T. (2005, September 30). What Is Web 2.0: Design Patterns and Business Models for the Next Generation of Software. Retrieved from http://www.oreilly.com/pub/a/web2/archive/what-is-web-20.html\#_federated=1.

Ortlipp, M. (2008). Keeping and Using Reflective Journals in the Qualitative Research Process. The Qualitative Report, 13(4), 695-705.

Papacharissi, Z. (2002). The Virtual Sphere: The Internet as a Public Sphere. New Media \& 
Society, 4(1), 9-27.

Papacharissi, Z. (2009). Journalism and Citizenship: New Agendas in Communication.

New York: Routledge.

Papacharissi, Z. (2013). A Private Sphere: Democracy in a Digital Age. New Jersey: John Wiley \& Sons.

Pateman, C. (1988). The Sexual Contract. Cambridge: Polity Press.

Poster, M. (2001). What's the Matter with the Internet? Minneapolis: University of Minnesota Press.

Prince, J. (2004). Keeping the Conversation Going: Voluntary Associations in the Public Sphere(s). Media International Australia Incorporating Culture and Policy, 111, $145-157$.

Provost, C. (2013, May 31). Bill Gates and Dambisa Moyo Spat Obscures the Real Aid Debate. Retrieved from http://www.theguardian.com/global-development/povertymatters/2013/may/31/bill-gates-dambisa-moyo-aid.

Quan-Haase, A., \& Martin, K. (2013). Digital Curation and the Networked Audience of Urban Events: Expanding La Fiesta de Santo Tomas from the Physical to the Virtual Environment. The International Communication Gazette, 00(0), 1-17.

Raboy, M., \& Shtern, J. (2010). Media Divides: Communication Rights and the Right to Communicate in Canada. Vancouver: UBC Press.

Rachels, J., \& Rachels, S. (2011). The Elements of Moral Philosophy. New York: McGrawHill.

Radovanovic, D. (2013). The Internet and Digital Divide in South Eastern Europe: Connectivity does not end the Digital Divide, Skills do. In Ragnedda, M. and 
Muschert, G.W. (Eds.), The Digital Divide (pp. 165-176). London: Routledge. Ragnedda, M. and Muschert, G.W. (2013). The Digital Divide. London: Routledge. Remaley, M. H., \& Dineen, P. (2007). Constructing a Modern Democracy, One Forum at a Time. National Civic Review, Summer, 42-48.

Robinson, L. \& Schulz, J. (2011). New Fieldsites, New Methods: New Ethnographic Opportunities. In S. N. Hesse-Biber (Ed.), The Handbook of Emergent Technologies in Social Research (pp. 180-198). New York: Oxford University Press, Inc.

Schirato, T. \& Webb, J. (2003). Bourdieu's Concept of Reflexivity as Metaliteracy. Cultural Studies, 17(3/4), 539-52.

Schirato, T., Danaher, G., \& Webb, J. (2012). Understanding Foucault: a Critical Introduction. Sydney: Allen \& Unwin.

Shao, P. \& Wang, Y. (2016). How Does Social Media Change Chinese Political Culture? The Formation of Fragmentized Public Sphere. Telematics and Informatics, 1-11.

Shim, H., You, K.H., Lee, J.K., \& Go, E. (2015). Why do People Access News with Mobile Devices? Exploring the Role of Suitability Perception and Motives on Mobile News Use. Telematics and Informatics, 32, 108-117.

Silverstone, R. (2007). On the Rise of the Mediapolis. Cambridge: Polity.

Smith, L. T. (1999). Decolonizing Methodologies: Research and Indigenous Peoples. New York: St. Martin's Press.

Steinmetz, K. F. (2012). Message Received: Virtual Ethnography in Online Message Boards. International Journal of Qualitative Methods, 11(1), 26-39.

Stephenson, H. (2015, November 14). Digital Inclusion: What is it and Why is it Important? Retrieved from http://www.itproportal.com/2015/11/14/digital- 
inclusion-what-is-it-and-why-is-it-important/\#ixzz3rbOpyN1O.

Swift, J., Davies, J. M., Clarke, R. G., \& Czerny, M. S. J. (2004). Getting Started on Social Analysis in Canada. In W. K. Carol (Ed.), Critical Strategies for Social Research (pp. 116-124). Toronto: Canadian Scholars' Press Inc.

Tilley, E. (2005). Media Relations. In F. Sligo and R. Bathurst (Eds.), Communication in the New Zealand Workplace: Theory and Practice (pp. 145-160). Wellington: Software Technology N. Z. Ltd.

Townsend, L. (2015). Rural Crafting Communities in the Digital Age. Working Papers of the Communities \& Cultures Network+, 6. Retrieved from http://www.communitiesandculture.org/projects/rural-crafting-communities-in-thedigital-age/.

Tully, J. (2013). Chapter 9. On the Global Multiplicity of Public Spheres: the Democratic Transformation of the Public Sphere? In C.J. Emden \& D. Midgley (Eds.), Beyond Habermas: Democracy, Knowledge, and the Public Sphere (pp. 169-204). U.S.: Berghan Books.

Turkle, S. (1995). Life on the Screen: Identity in the Age of the Internet. New York: Touchstone.

Ugarteche, O. (2007). Transnationalizing the Public Sphere: A Critique of Fraser. Theory, Culture \& Society, 24(4), 65-69. http://doi.org/10.1177/0263276407080095.

Van Dusen, J. (2015, March 18). Peter Goldring's Pocket Pen Video Camera Keeps MPs Talking. Retrieved from http://www.cbc.ca/1.2999850.

Volkmer, I. (2007). Governing the "Spatial Reach"? Spheres of Influence and Challenges of Global Media Policy. International Journal of Communication, 1, 56-73. 
Volkmer, I. (2008). Conflict-Related Media Events and Cultures of Proximity. Media, War \& Conflict, 1(1), 90-98.

Volkmer, I. (2014). The Global Public Sphere: Public Communication in the Age of Reflective Interdependence. London: Sage.

Warner, M. (2002). Publics and Counterpublics (Abbreviated Version). Quarterly Journal of Speech, 88(4), 413-425.

Watt, D. (2007). On Becoming a Qualitative Researcher. The Qualitative Report, 12(1), 82-101.

Wellman, B., Quan-Haase, A., Witte, J., \& Hampton, K. (2001). Does the Internet Increase, Decrease, or Supplement Social Capital? Social Networks, Participation, and Community Commitment. American Behavioral Scientist, 45(3), 436-455.

Wilson, R. (2013). Public Service and Political Experience. In C. J. Emden \& D. Midgley (Eds.), Beyond Habermas: Democracy, Knowledge, and the Public Sphere (pp. 1928). U.S.: Berghan Books.

Women's Rights Online: Translating Access into Empowerment. (2015, October 20). Retrieved from http://webfoundation.org/about/research/womens-rights-online$2015 /$.

World, P. (2012, December 8). World Pulse. Retrieved from http:www.worldpulse.com Zizek, S. (1997). The Plague of Fantasies. London: Verso. 
WorldPulse.com Journals

Ending Gender-Based Violence 2012 (EGBV)

'This Call to Action Ended in 2012. World Pulse. https://www.worldpulse.com/en/getinvolved/take-action/199. Web. 11 August 2015.

1

Tessie, “Some Women Do Have Them.......3.” World Pulse. 26 November 2012. https:/www.worldpulse.com/en/community/users/tessie/posts/22986. Web. 11 August 2015.

2

Tressa Kentner, "Education and economic empowerment are key to ending violence against women.” World Pulse. 25 November 2012.

https://www.worldpulse.com/en/community/users/tressa-kentner/posts/22980. Web. 11 August 2015.

3

mitukhurana, "Dr. Mitu Khurana: the defender of India's baby girls." World Pulse. 25 November 2012.

https://www.worldpulse.com/en/community/users/mitukhurana/posts/22979. Web. 11 August 2015. 
LatiNegra, "Violence \& Silence: The Gravity." World Pulse. 25 November 2012. https://www.worldpulse.com/en/community/users/latinegra/posts/22975. Web. 11 August 2015.

5

kotravai, "A teenager girl at the age of 13 has been sexually exploited and tortured." World Pulse. 25 November 2012.

https://www.worldpulse.com/en/community/users/kotravai/posts/22972. Web. 11 August 2015.

6

LatiNegra, "For the Woman Staring at Her Mother in Her Reflection:." World Pulse. 25

November 2012. https://www.worldpulse.com/en/community/users/latinegra/posts/22971. Web. 11 August 2015.

7

yvonne, “Training for Violence Against Women.” World Pulse. 25 November 2012.

https://www.worldpulse.com/en/community/users/yvonne/posts/22968. Web. 11 August 2015. 
ster2109, "La violencia estetica: una nueva forma de violencia contra la mujer [The aesthetic violence: a new form of violence against women]." World Pulse. 24 November 2012. https://www.worldpulse.com/en/community/users/ster2109/posts/22966. Web. 11 August 2015.

9

Noorjahan Akbar, “A Letter to My Harasser.” World Pulse. 24 November 2012. https://www.worldpulse.com/en/community/users/noorjahan-akbar/posts/22963. Web. 11 August 2015.

10

Lea, “A play on domestic abuse in Vietnam.” World Pulse. 23 November 2012. https://www.worldpulse.com/en/community/users/lea/posts/22956. Web. 11 August 2015.

Lubala Rosalie, "Violences Faites Aux Viellards En RDC [Violence Against Old Men in DRC]." World Pulse. 30 October 2012.

https://www.worldpulse.com/en/community/users/lubala-rosalie/posts/22513. Web. 11 August 2015.

12

Hamisi Namanvu, “Les Mamans Ont Droit d'etre forme en Francais [Moms Right to be Shaped French].” World Pulse. 30 October 2012. 
https://www.worldpulse.com/en/community/users/hamisi-namanvu/posts/22509. Web. 11 August 2015.

13

Michelline Kadorho, “Les Violences Faites Aux Malades et aux Infirmiers [Violence against Patients and Nurses].” World Pulse. 30 October 2012.

https://www.worldpulse.com/en/community/users/michelline-kadorho/posts/22508. Web.

11 August 2015.

14

Riziki Bisonga, "Violence de droit e la Femme [Law of Violence of Women]." World Pulse. 30 October 2012. https://www.worldpulse.com/en/community/users/rizikibisonga/posts/22507. Web. 11 August 2015.

15

Anuarite, "Violences Des Droits de la Femme [Violence on Women's Rights]." World Pulse. 30 October 2012.

https://www.worldpulse.com/en/community/users/anuarite/posts/22423. Web. 11 August 2015.

16 
Neema, "From Congo: We Want Our Hero Back, NOW!" World Pulse. 29 October 2012. https://www.worldpulse.com/en/community/users/neema/posts/22441. Web. 11 August 2015.

Antego2010, "The African Woman: Breaking the Stereotype." World Pulse. 29 October 2012. https://www.worldpulse.com/en/community/users/antego2010/posts/22497. Web. 11 August 2015.

18

Lobna Sedky, "The Latest Developments of the Case of Sexual Harassment in Cairo." World Pulse. 29 October 2012 https://www.worldpulse.com/en/community/users/lobnasedky/posts/22493. Web. 11 August 2015.

19

Michelline Kadorho, "Les Violences faites aux agents de 1 etat [Violence against Agents of the State]." World Pulse. 29 October 2012.

https://www.worldpulse.com/en/community/users/michelline-kadorho/posts/22491. Web. 11 August 2015. 
Namavu, "Il’Insecurite En R D Congo." World Pulse. 29 October 2012.

https://www.worldpulse.com/en/community/users/namavu/posts/22490. Web. 11 August 2015.

Valéria Barbosa da Silva, "I am not an Object to be Owned. I'm only one Person and I Deserve to be Loved. World Pulse. 04 October 2012.

https://www.worldpulse.com/en/community/users/valeria-barbosa-da-silva/posts/22258.

Web. 11 August 2015.

22

Sibatu Nkwana, "Pourquoi la Souffrance dans la Vie? [Why the Suffering in Life?]." World Pulse. 03 October 2012. https://www.worldpulse.com/en/community/users/sibatunkwana/posts/22254. Web. 11 August 2015.

23

Mukut, "Street Sexual Harassment: I refuse to be a Victime; I refuse to take the Blame." World Pulse. 03 October 2012.

https://www.worldpulse.com/en/community/users/mukut/posts/22253. Web. 11 August 2015. 
EK. Chemorion, "Habitual Rape of Female Students by Neighbouring Village: VAW." World Pulse. 03 October 2012. https://www.worldpulse.com/en/community/users/ekchemorion/posts/22251. Web. 11 August 2015.

Kotravai, "Man Kills Wife for Want of Sex \# VAW.” World Pulse. 02 October 2012. https://www.worldpulse.com/en/community/users/kotravai/posts/22250. Web. 11 August 2015.

Oceaser, "Violence." World Pulse. 01 October 2012. https://www.worldpulse.com/en/community/users/oceaser/posts/22246. Web. 11 August 2015.

27

Madeleine Bwenge, "Leadership and Empowerment.” World Pulse. 01 October 2012. https://www.worldpulse.com/en/community/users/madeleine-bwenge/posts/22242. Web. 11 August 2015.

EK. Chemorion, "No More Customary Tears - I Want to be Myself and I have Chosen to be me.” World Pulse. 01 October 2012. 
https://www.worldpulse.com/en/community/users/ek-chemorion/posts/22239. Web. 11 August 2015 .

29

Valéria Barbosa da Silva, "A Woman, no Address, no Name and too Afraid to Live!!"

World Pulse. 30 September 2012.

https://www.worldpulse.com/en/community/users/valeria-barbosa-da-silva/posts/22236.

Web. 11 August 2015.

30

Ntakebuka Wilhelmine, "Le Viol Doit Etre Eradique [Rape Must be Eradicated].” World Pulse. 29 September 2012. https://www.worldpulse.com/en/community/users/ntakebukawilhelmine/posts/22234. Web. 11 August 2015.

31

Adele Wasolu, “Une Violence [Violence].” World Pulse. 17 September 2012. https://www.worldpulse.com/en/community/users/adele-wasolu/posts/22088. Web. 11 August 2015 .

32

Sandra Omba, "Cout de transport en Republique Democratique du Congo (RDC) [Transportation Cost in Democratic Republic of Congo (DRC)]." World Pulse. 17 
September 2012. https://www.worldpulse.com/en/community/users/sandraomba/posts/22086. Web. 11 August 2015.

33

Mambo Filomene, "Proposition [Proposition]." World Pulse. 17 September 2012.

https://www.worldpulse.com/en/community/users/mambo-filomene/posts/22085. Web. 11 August 2015.

34

Madeleine Bwenge, “Gender Based Violence.” World Pulse. 17 September 2012.

https://www.worldpulse.com/en/community/users/madeleine-bwenge/posts/22083. Web. 11 August 2015.

35

Madeleine Bwenge, "Leadership and Empowerment." World Pulse. 17 September 2012. https://www.worldpulse.com/en/community/users/madeleine-bwenge/posts/22082. Web. 11 August 2015.

36

Betty Makoni, “Zimbabwean Women Break Silence on Alleged Rapist in UK-Betty Makoni Radio Show.” World Pulse. 16 September 2012. https://www.worldpulse.com/en/community/users/betty-makoni/posts/22076. Web. 11 August 2015. 
Wendyiscalm, "How to Develop Inner Empowerment when External Power is making Life Tough.” World Pulse. 16 September 2012.

https://www.worldpulse.com/en/community/users/wendyiscalm/posts/22074. Web. 11 August 2015.

38

Hilda Mary, “Change Happens.” World Pulse. 15 September 2012. https://www.worldpulse.com/en/community/users/hilda-mary/posts/22070. Web. 11 August 2015.

39

Innocency Massanja, “Ending Gender-Based Violence.” World Pulse. 15 September 2012. https://www.worldpulse.com/en/community/users/innocency-massanja/posts/22066. Web. 11 August 2015.

40

Brigitte Mawazo Kyalondawa, "La Viol a Domicile [The Rape Home].” World Pulse. 14 September 2012. https://www.worldpulse.com/en/community/users/brigitte-mawazokyalondawa/posts/22062. Web. 11 August 2015. 
Brigitte Mawazo Kyalondawa, "Economie Domestique [Home Economics]." World Pulse. 05 September 2012. https://www.worldpulse.com/en/community/users/brigitte-mawazokyalondawa/posts/21909. Web. 11 August 2015.

Aminalydia, "La Violence Basé Sous Le Genre [Violence based on Gender]." World Pulse. 04 September 2012.

https://www.worldpulse.com/en/community/users/aminalydia/posts/21897. Web. 11 August 2015.

43

Yvonne Mushayuma, "Genre et Violence [Gender and Violence].” World Pulse. 04

September 2012. https://www.worldpulse.com/en/community/users/yvonnemushayuma/posts/21894. Web. 11 August 2015.

44

Kabene, "Nous Avons Aussi Nos Droits! [We Also Have Our Rights]." World Pulse. 04

September 2012. https://www.worldpulse.com/en/community/users/kabene/posts/21893.

Web. 11 August 2015.

45

Hamisi Namanvu, "Les Mamans Dans Leurs Famille Elargie [Moms in their Extended Family]." World Pulse. 04 September 2012. 
https://www.worldpulse.com/en/community/users/hamisi-namanvu/posts/21892. Web. 11 August 2015.

46

Madeleine Bwenge, “Leadership and Empowerment.” World Pulse. 04 September 2012. https://www.worldpulse.com/en/community/users/madeleine-bwenge/posts/21890. Web. 11 August 2015.

47

Kotravai, “Update on Kaliammal's Case.” World Pulse. 04 September 2012.

https://www.worldpulse.com/en/community/users/kotravai/posts/21887. Web. 11 August 2015.

48

Brigitte Mawazo Kyalondawa, "La Femme Rurale est au Centre de la Vitalite de la Famille [Rural Women Are the Centre of the Vitality of the Family].” World Pulse. 03 September 2012. https://www.worldpulse.com/en/community/users/brigitte-mawazokyalondawa/posts/21883. Web. 11 August 2015.

49

Brigitte Mawazo Kyalondawa, “Ce Que le Monde ne Sait Pas [What the World Do Not Know]." World Pulse. 03 September 2012. 
https://www.worldpulse.com/en/community/users/brigitte-mawazo-

kyalondawa/posts/21882. Web. 11 August 2015.

50

Ruhebuza Vumilia Jeannette, "Violence faite aux Femmes Congolaises [Violence against Congolese Women].” World Pulse. 03 September 2012.

https://www.worldpulse.com/en/community/users/ruhebuza-vumilia-jeannette/posts/21881.

Web. 11 August 2015.

51

Japalouise, "Difficulté d'une Enseignante [Difficulty of a Teacher]." World Pulse. 18 July 2012. https://www.worldpulse.com/en/community/users/japalouise/posts/21261. Web. 11 August 2015.

52

Nabintu, "La Viw Sexuelle en Famille [Sexual Family Life]." World Pulse. 18 July 2012. https://www.worldpulse.com/en/community/users/nabintu/posts/21260. Web. 11 August 2015.

53

Japalouise, "Les Difficultés d'une Femme [The Difficulties of a Woman]." World Pulse. 18 July 2012. https://www.worldpulse.com/en/community/users/japalouise/posts/21259. Web. 11 August 2015. 
Kotravai, "Women as Object of all time Threat.” World Pulse. 17 July 2012.

https://www.worldpulse.com/en/community/users/kotravai/posts/21247. Web. 11 August 2015.

55

Mukuzo Mamy, ‘Promontion de Genre.” World Pulse. 17 July 2012.

https://www.worldpulse.com/en/community/users/mukuzo-mamy/posts/21241. Web. 11 August 2015.

56

Mumbalima, “Autres Difficultés Rencontrées dans notre Association UFD Union des Femmes pour le Développement [Other Difficulties Encountered in Our UFD Union Association of Women for Development].” World Pulse. 17 July 2012. https://www.worldpulse.com/en/community/users/mumbalima/posts/21237. Web. 11 August 2015.

57

Madeleine Bwenge, "Politics and Society.” World Pulse. 17 July 2012.

https://www.worldpulse.com/en/community/users/madeleine-bwenge/posts/21236. Web. 11 August 2012. 
Madeleine Bwenge, “Gender-Based Violence.” World Pulse. 17 July 2012.

https://www.worldpulse.com/en/community/users/madeleine-bwenge/posts/21233. Web.

11 August 2015.

59

Nabintu, "La vie Familliale [Life Familliale].” 16 July 2012.

https://www.worldpulse.com/en/community/users/nabintu/posts/21224. Web. 11 August 2015.

60

Madeleine Bwenge, “Gender-Based Violences.” World Pulse. 13 July 2012.

https://www.worldpulse.com/en/community/users/madeleine-bwenge/posts/21199. Web.

11 August 2015.

61

Innocency Massanja, "Violence Against Women and My Life." World Pulse. 10 June 2012. https://www.worldpulse.com/en/community/users/innocency-massanja/posts/20845. Web. 11 August 2015.

Girls Transform The World 2013 (GTTW) 
'This Call to Action Ended August 2013.' World Pulse.

https://www.worldpulse.com/en/get-involved/take-action/228. Web. 13 August 2015.

1

Abby Kakiyes, “Our Reality.” World Pulse. 09 May 2013.

https://www.worldpulse.com/en/community/users/abby-kakiyes/posts/26194. Web. 13 August 2015.

2

Dianne Scott, “A Letter to my Mother.” World Pulse. 09 May 2013.

https://www.worldpulse.com/en/community/users/dianne-scott/posts/26193. Web. 13 August 2015.

3

Hekima Place, "Education: the Gift that Keeps on Giving." World Pulse. 09 May 2013. https://www.worldpulse.com/en/community/users/hekima-place/posts/26191. Web. 13 August 2015.

4

Amiesissoho, "Even in Prison, you made me realize we have rights! In Honour of Dr. Isatou Touray." World Pulse. 09 May 2013. https://www.worldpulse.com/en/community/users/amiesissoho/posts/26189. Web. 13 August 2015. 
Sutanuka Banerjee, "Countering Gender Bias: "Boys Have Trucks - Girls Have Dolls." World Pulse. 09 May 2013. https://www.worldpulse.com/en/community/users/sutanukabanerjee/posts/26185. Web. 13 August 2015.

6

Mugisha Solange, "Tout Est Possible a une fille Couragieuse [Everything is possible for a Girl Couragieuse]." World Pulse. 09 May 2013.

https://www.worldpulse.com/en/community/users/mugisha-solange/posts/26183. Web. 13 August 2015.

7

Sangita Thapa, “To My Mother, My Hero.” World Pulse. 08 May 2013.

https://www.worldpulse.com/en/community/users/sangita-thapa/posts/26171. Web. 13 August 2015.

8

Mulheres Republicanas de Mozambique, "School Girls and Women Entrepreneur of Mozambique." World Pulse. 08 May 2013. https://www.worldpulse.com/en/community/users/mulheres-republicanas-demozambique/posts/26168. Web. 13 August 2015. 
Chargerrose7, “To My Daughters.” World Pulse. 08 May 2013.

https://www.worldpulse.com/en/community/users/chargerrose7/posts/24868. Web. 13 August 2015.

10

Hillary24, "Providing Young Girls with Defence Awareness Tactics may be ideal in this day and age!" World Pulse. 07 May 2013.

https://www.worldpulse.com/en/community/users/hillary24/posts/26157. Web. 13 August 2015.

11

Rhoda Robinson, "School or No School? That is the Question.” World Pulse. 22 April 2013. https://www.worldpulse.com/en/community/users/rhoda-robinson/posts/25678. Web. 13 August 2015.

12

Walaa Salah, "It's the Combination of Religious Fundamentalism and Discriminary Laws." World Pulse. 22 April 2013. https:/www.worldpulse.com/en/community/users/walaasalah/posts/25677. Web. 13 August 2015.

13 
Amina_3, "The Ultimate Game-Changer for the Persevering Souls." World Pulse. 22 April 2013. https://www.worldpulse.com/en/community/users/amina3/posts/25676. Web. 13 August 2015.

14

Paulina Lawsin, “Aniluap, Princess of the Beach.” World Pulse. 22 April 2013. https://www.worldpulse.com/en/community/users/paulina-lawsin/posts/25672. Web. 13 August 2015.

15

MyrtleG, "Our Children are our Future; Let's Listen and Teach Them Well!" World Pulse. 22 April 2013. https://www.worldpulse.com/en/community/users/myrtleg/posts/25670.

Web. 13 August 2015.

16

Nicole Musimwa, "Overcoming Barriers to a Girl's Education in Congo.” World Pulse. 22 April 2013. https://www.worldpulse.com/en/community/users/nicolemusimwa/posts/25668. Web. 13 August 2015.

17

Rebecca R, “Nanyonga Rehema: Celebrating and Uplifting Girl in Real Life.” World Pulse. 22 April 2013. https://www.worldpulse.com/en/community/users/rebecca-r/posts/25667. Web. 13 August 2015. 
Jampa, “My Peers were kept at Home at that Time.” World Pulse. 22 April 2013.

https://www.worldpulse.com/en/community/users/jampa/posts/25666. Web. 13 August 2015.

19

Ansupokharel, "Multifaceted Society and Education: Somewhat Complicated!!” World Pulse. 22 April 2013.

https://www.worldpulse.com/en/community/users/ansupokharel/posts/25664. Web. 13 August 2015.

20

Helwa, "I Am the Loudspeaker for Girls Education.” World Pulse. 22 April 2013. https://www.worldpulse.com/en/community/users/helwa/posts/25662. Web. 13 August 2015.

21

Tash, "Brew that Saved Her Life." World Pulse. 18 April 2013.

https://www.worldpulse.com/en/community/users/tash/posts/25480. Web. 13 August 2015.

22 
Gloriabit, “Mining Disaster.” World Pulse. 18 April 2013.

https://www.worldpulse.com/en/community/users/gloriabit/posts/25473. Web. 13 August 2015.

Chargerrose7, "Breaking Down Education Barriers.” World Pulse. 18 April 2013.

https://www.worldpulse.com/en/community/users/chargerrose7/posts/25468. Web. 13 August 2015.

24

Neelia Seyer, "Woman... Hitch Your Wagon to a Star." World Pulse. 17 April 2013. https://www.worldpulse.com/en/community/users/neelia-seyer/posts/25464. Web. 13 August 2015.

Nakinti, "When Poverty Strikes, Education is at Risk; 4 Young Girls Skipped Classes to Raise Money for Others Education!" World Pulse. 17 April 2013. https://www.worldpulse.com/en/community/users/nakinti/posts/25462. Web. 13 August 2015.

26

TJ, “Books First...Boys Later...” World Pulse. 17 April 2013.

https://www.worldpulse.com/en/community/users/tj/posts/25456. Web. 13 August 2015. 
Latinegra, "The Case for Supporting Young Women.” World Pulse. 17 April 2013.

https://www.worldpulse.com/en/community/users/latinegra/posts/25453. Web. 13 August 2015.

28

Bitani, "VOF Assignment Week 3/Girl’s Education.” World Pulse. 17 April 2013.

https://www.worldpulse.com/en/community/users/bitani/posts/25436. Web. 13 August 2015.

29

Caryn Dudarevitch, “Zephaniah Free Education: An Interview.” World Pulse. 16 April 2013. https://www.worldpulse.com/en/community/users/caryn-dudarevitch/posts/25430.

Web. 13 August 2015.

30

Aminah, "Mother-Machine my Strength!” World Pulse. 16 April 2013.

https://www.worldpulse.com/en/community/users/aminah/posts/25422. Web. 13 August 2015.

31 
Diane Dillon, “One Step at a Time.” World Pulse. 28 March 2013.

https://www.worldpulse.com/en/community/users/diane-dillon/posts/24514. Web. 13 August 2015.

Bagirathi Ramanathan, “Girls Education and Empowerment.” World Pulse. 28 March 2013. https://www.worldpulse.com/en/community/users/bagirathi-ramanathan/posts/24509. Web. 13 August 2015.

33

Sophie, “Outliers, Luck or Fate?” World Pulse. 27 March 2013. https://www.worldpulse.com/en/community/users/sophie/posts/24472. Web. 13 August 2015.

34

Tadzie, “Introducing Myself and My Journal: Englightened.” World Pulse. 27 March 2013. https://www.worldpulse.com/en/community/users/tadzie/posts/24457. Web. 13 August 2015.

35

Nyaradzayi, "Where Does the Bus Come From?” World Pulse. 26 March 2013. https://www.worldpulse.com/en/community/users/nyaradzayi/posts/24439. Web. 13 August 2015. 
Hend Ali, “الو اقع جدار من كسرة] [Fragment of a Wall of Reality.” World Pulse. 18 March 2013. https:/www.worldpulse.com/en/community/users/hend-ali/posts/24270. Web. 13 August 2015.

Lea, "Interview with an Afghan Student About the Situation for Women in Afghanistan." World Pulse. 11 March 2013.

https://www.worldpulse.com/en/community/users/lea/posts/24166. Web. 13 August 2015.

Women Weave The Web 2014 (WWTW)

'This Campaign Ended August 2014.' World Pulse. https://www.worldpulse.com/en/getinvolved/take-action/250. Web. 14 August 2015.

1

Melva Sangri, "El ABC Digital que Salva Vidas [The Digital ABC Saving Lives]." World Pulse. 07 August 2014. https://www.worldpulse.com/en/community/users/melvasangri/posts/33978. Web. 14 August 2015. 
Miria, "The Nakawa Community Women's Digital Statement firms the ground for embracing the Internet - Kampala, Uganda.” World Pulse. 07 August 2014. https://www.worldpulse.com/en/community/users/miria/posts/33976. Web. 14 August 2015.

Women on the Rising, "The Very Importance of Digitally Empowering Women.” World Pulse. 07 August 2014. https://www.worldpulse.com/en/community/users/womenrising/posts/33973. Web. 14 August 2015.

4

Women on the Rising, "Online Power, Offline Ability and Guts." World Pulse. 07 August 2014. https://www.worldpulse.com/en/community/users/women-rising/posts/33972. Web. 14 August 2015.

5

Women on the Rising, "I Love the Web: The Genesis.” World Pulse. 07 August 2014. https://www.worldpulse.com/en/community/users/women-rising/posts/33971. Web. 14 August 2015.

6

Gladys Shumbambiri, "Digital Tools and Women in Zimbabwe." World Pulse. 07 August 2014. https://www.worldpulse.com/en/community/users/gladys-shumbambiri/posts/33969. Web. 14 August 2015. 
Gladys Shumbambiri, "Digitally Empower the Woman to Transform the Nation and the World.” World Pulse. 07 August 2014.

https://www.worldpulse.com/en/community/users/gladys-shumbambiri/posts/33967. Web. 14 August 2015.

8

Carolepng, “Empowered Online to Bring Impact Offline!’ World Pulse. 06 August 2014. https://www.worldpulse.com/en/community/users/carolepng/posts/33960. Web. 14 August 2015.

9

Rasheedat Sanni, "Educating the Women, Means Educating the World." World Pulse. 06 August 2014. https://www.worldpulse.com/en/community/users/rasheedatsanni/posts/33956. Web. 14 August 2015.

10

Kiapi K. Freddie, "Re: Empowering Girls in Uganda with IT Skills.” World Pulse. 06 August 2014. https://www.worldpulse.com/en/community/users/kiapi-kfreddie/posts/33955. Web. 14 August 2015. 
Sherna Alexander, "Why Women Should be Digitally Educated." World Pulse. 03 July 2014. https://www.worldpulse.com/en/community/users/sherna-alexander/posts/33540. Web. 14 August 2015.

Garima, "Can Africa use Technology to Reverse the Cycle of Poverty and Achieve Economic Growth?" World Pulse. 03 July 2014.

https://www.worldpulse.com/en/community/users/garima/posts/33534. Web. 14 August 2015.

13

AlejandraL, "Internet: Una Herramienta Indispensable Para El Desarrollo Humano [Internet: an Indispensable Tool for Human Development].” World Pulse. 02 July 2014. https://www.worldpulse.com/en/community/users/alejandral/posts/33529. Web. 14 August 2015.

14

Anakarenart, "The World Needs Latin America and Mexico Women in Tech." World Pulse. 02 July 2014. https://www.worldpulse.com/en/community/users/anakarenart/posts/33528. Web. 14 August 2015. 
Anakarenart, "Epic Queen: Empowering Myself and Empowering Others Through

Technology." World Pulse. 02 July 2014.

https://www.worldpulse.com/en/community/users/anakarenart/posts/33525. Web. 14

August 2015.

16

Carolepng, "YoBloCo - Youth Blogging Competition 2013/14." World Pulse. 02 July 2014. https://www.worldpulse.com/en/community/users/carolepng/posts/33519. Web. 14 August 2015.

17

Moshelbeads, "Why do Many Women Have Mobile Phones in Africa and yet a few use the Internet?" World Pulse. 01 July 2014.

https://www.worldpulse.com/en/community/users/moshelbeads/posts/33502. Web. 14 August 2015.

18

Moshelbeads, "Empowering Women Through the Internet." World Pulse. 01 July 2014. https://www.worldpulse.com/en/community/users/moshelbeads/posts/33499. Web. 14 August 2015.

19 
Diane Dillon, "First Edition.” World Pulse. 30 June 2014.

https://www.worldpulse.com/en/community/users/diane-dillon/posts/33492. Web. 14 August 2015.

20

Moshelbeads, "How the Internet Empowered me to Start a Business." World Pulse. 30 June 2014. https://www.worldpulse.com/en/community/users/moshelbeads/posts/33491. Web. 14 August 2015.

21

Iffat Gill, "Pakistan: Lack of Awareness about ICTs use and its Impacts on Young Girls." World Pulse. 27 May 2014. https://www.worldpulse.com/en/community/users/iffatgill/posts/33027. Web. 14 August 2015.

Sanjana Shrestha, "Digital Tools Raise Awareness about Maternal Health in Nepal.” World Pulse. 27 May 2014. https://www.worldpulse.com/en/community/users/sanjanashrestha/posts/33024. Web. 14 August 2015.

Rebuild Sakubva, "Supporting Women Empowerment Through the Web." World Pulse. 27 May 2014. https://www.worldpulse.com/en/community/users/rebuild-sakubva/posts/33017. Web. 14 August 2015. 
Begeziani, “La Femme et La Technologie de L’Information Delamanque du Courant [Women and Technology Information Current Delamanque].” World Pulse. 27 May 2014. https:/www.worldpulse.com/en/community/users/begeziani/posts/33016. Web. 14 August 2015.

Neeme Ortance, “La Vie et la Technologie [Life and Technology].” World Pulse. 26 May 2014. https://www.worldpulse.com/en/community/users/neeme-ortance/posts/32999. Web. 14 August 2015.

26

Carocimador, “Women's Access to Financial Services Empower Women / World Pulse Post.” World Pulse. 25 May 2014.

https://www.worldpulse.com/en/community/users/carocimador/posts/32992. Web. 14 August 2015.

Carocimador, "Great Resource for our Digital and Financial Empowerment." World Pulse. 25 May 2014. https://www.worldpulse.com/en/community/users/carocimador/posts/32990. Web. 14 August 2015. 
Melissa_Banigan, “Global Leadership and Empowerment Summit for Teen Girls and Women in Peru!" World Pulse. 24 May 2014.

https://www.worldpulse.com/en/community/users/melissabanigan/posts/32979. Web. 14 August 2015.

29

Adahmbah, "Virtual Learning Tools for Victims of Teenage Pregnancies in Rural Communities." World Pulse. 24 May 2014.

https://www.worldpulse.com/en/community/users/adahmbah/posts/32978. Web. 14 August 2015.

30

Rebuild Sakubva, "Supporting Economic Empowerment Through the Web." World Pulse. 23 May 2014. https://www.worldpulse.com/en/community/users/rebuildsakubva/posts/32951. Web. 14 August 2015.

31

Mauri, "Digital Literacy: Might Lightweight Technology and Proper Help Lower the Stick?" World Pulse. 03 April 2014. https://www.worldpulse.com/en/community/users/mauri/posts/32333. Web. 14 August 2015. 
Susana Khabbaz, "What is Equality?” World Pulse. 03 April 2014.

https://www.worldpulse.com/en/community/users/susana-khabbaz/posts/32331. Web. 14 August 2015.

33

Sista-Kenya, “Grass Roots Women in Digital Age.” World Pulse. 02 April 2014.

https://www.worldpulse.com/en/community/users/sista-kenya/posts/32320. Web. 14 August 2015.

34

Kkibaara, "The Challenged Relationship Between the Woman and Digital Technology." World Pulse. 02 April 2014.

https://www.worldpulse.com/en/community/users/kkibaara/posts/32316. Web. 14 August 2015.

35

Namasomo Clémentine Kiyobe, "La Nouvelle Technologie [New Technology].” World Pulse. 02 April 2014. https://www.worldpulse.com/en/community/users/namasomoclementine-kiyobe/posts/32314. Web. 14 August 2015. 
Bajira Chishuba, “Ce Qui M’Avait Pousser a la Formation de la Nouvelle Technologie/L'Internet [What Had me Push the Formation of New Technology/Internet].” World Pulse. 02 April 2014. https://www.worldpulse.com/en/community/users/bajirachishuba/posts/32311. Web. 14 August 2015.

37

Iffat Gill, "ChunriChoupaal - a Meeting Place for Women to get Access, Learn and Connect." World Pulse. 31 March 2014.

https://www.worldpulse.com/en/community/users/iffat-gill/posts/32285. Web. 14 August 2015.

38

Kujamac12, "Internet Access and a Breakthrough in Many Folds Part 2." World Pulse. 31 March 2014. https://www.worldpulse.com/en/community/users/kujamac12/posts/32284. Web. 14 August 2015.

39

Nisha Pahuja, “The World Before Us.” World Pulse. 31 March 2014.

https://www.worldpulse.com/en/community/users/nisha-pahuja/posts/32283. Web. 14 August 2015.

40 
Melissa_Banigan, “The Advice Project - Empowering Young Women Around the Globe via the Internet and Education.” World Pulse. 31 March 2014.

https://www.worldpulse.com/en/community/users/melissabanigan/posts/32282. Web. 14 August 2015.

41

Siatta, "WWW." World Pulse. 11 March 2014.

https://www.worldpulse.com/en/community/users/siatta/posts/31871. Web. 14 August 2015.

42

Adife Sudkivu, "La Femme et la Technologie [Women and Technology]." World Pulse. 11 March 2014. https://www.worldpulse.com/en/community/users/adife-sudkivu/posts/31867.

Web. 14 August 2015.

43

Masoso Antoinette, “Technology is Very Good to us.” World Pulse. 11 March 2014. https://www.worldpulse.com/en/community/users/masoso-antoinette/posts/31865. Web. 14 August 2015.

44

Paola, "Nuetra Historia se ha Centrado en Motivar el Espiritu de Descubrimiento en las Mujeres [Our History has Focused on Encouraging the Spirit of Discovery in Women].” World Pulse. 10 March 2014. 
https://www.worldpulse.com/en/community/users/paola/posts/31861. Web. 14 August 2015.

45

Nabiye Tal, "Challenges the Nigerian Women Face in Accessing the Web." World Pulse. 10 March 2014. https://www.worldpulse.com/en/community/users/nabiye-tal/posts/31854.

Web. 14 August 2015.

46

Martha Llano, "Digital Connection as Running Water." World Pulse. 09 March 2014. https://www.worldpulse.com/en/community/users/martha-llano/posts/31845. Web. 14 August 2015.

47

Gunu_K, "My Online Experience.” World Pulse. 09 March 2014.

https://www.worldpulse.com/en/community/users/gunuk/posts/31839. Web. 14 August 2015.

48

Amb. Paul Lengar, "Low Levels of ICT and Access to Social Media for Women and Young Girls in Sierra Leone.” World Pulse. 08 March 2014.

https://www.worldpulse.com/en/community/users/amb-paul-lengar/posts/31832. Web. 14 August 2015. 
Diane Dillon, “Olga's Story and the Digital Internet Movement with World Pulse.” World Pulse. 07 March 2014. https://www.worldpulse.com/en/community/users/dianedillon/posts/31817. Web. 14 August 2015.

50

Masoka, "L’Importance de la Nouvelle Technologie [The Importance of New Technology]." World Pulse. 06 March 2014.

https://www.worldpulse.com/en/community/users/masoka/posts/31792. Web. 14 August 2015.

51

Sanjay Dixit, “Empowerment of Women.” World Pulse. 16 January 2014. https://www.worldpulse.com/en/community/users/sanjay-dixit/posts/30945. Web. 14 August 2015 .

52

Y, “Appalachian Access.” World Pulse. 15 January 2014.

https://www.worldpulse.com/en/community/users/y/posts/30933. Web. 14 August 2015. 
Loyce, "Women Confront VAW using ICTs: Experiences from Remote and Fishing Community in Eastern Uganda.” World Pulse. 15 January 2014.

https://www.worldpulse.com/en/community/users/loyce/posts/30926. Web. 14 August 2015.

54

Kasindi Bulambo, “Femme et la Technologie de L'Informatique [Women and Computing Technology].” World Pulse. 15 January 2014.

https://www.worldpulse.com/en/community/users/kasindi-bulambo/posts/30923. Web. 14 August 2015.

55

Olutosin, "Weaving my Words and World on the Web." World Pulse. 15 January 2014. https://www.worldpulse.com/en/community/users/olutosin/posts/30921. Web. 14 August 2015.

56

SandyO, "What I Get Done for Women Would be Impossible without the WWWeb!" World Pulse. 14 January 2014.

https://www.worldpulse.com/en/community/users/sandyo/posts/30906. Web. 14 August 2015. 
Sister Zeph, “I Open Girls’ Minds.” World Pulse. 12 January 2014.

https://www.worldpulse.com/en/community/users/zephaniah/posts/30877. Web. 14 August 2015.

58

Stella Paul, "How I use Cell Phones to Bridge the Gap for the Voiceless." World Pulse. 06

January 2014. https://www.worldpulse.com/en/community/users/stella-paul/posts/30690.

Web. 14 August 2015. 


\section{Appendix}

\section{List of Abbreviations}

Digital Action Campaigns: DACs

Democratic Republic of Congo: DRC

Ending Gender-Based Violence: EGBV

Girls Transform The World: GTTW

Internet Service Providers: ISPs

Non-Governmental Organizations: NGOs

Voices of Our Future: VOF

Women Weave the Web: WWTW

World Pulse: WP

WorldPulse.com: WPc 


\section{Data Collection}

Appendix 1

\begin{tabular}{|l|l|l|l|}
\hline \multicolumn{3}{|c|}{ Data selection for the Digital Action Campaigns 2012-2014 } \\
\hline \multicolumn{1}{|c|}{ Campaign titles } & $\begin{array}{c}\text { Total \# of } \\
\text { journal entries } \\
\text { journal entries }\end{array}$ & $\begin{array}{c}\text { \# of comments } \\
\text { under selected } \\
\text { journal entries }\end{array}$ \\
\hline $\begin{array}{l}\text { Ending Gender-Based } \\
\text { Violence 2012 }\end{array}$ & 618 & 61 & 391 \\
\hline $\begin{array}{l}\text { Girls Transform The World } \\
2013\end{array}$ & 371 & 37 & 355 \\
\hline $\begin{array}{l}\text { Women Weave the Web } \\
2014\end{array}$ & 582 & 156 & 1274 \\
\hline Total & 1571 & 58 & 528 \\
\hline
\end{tabular}

Table 1: Data Selection for the Digital Action Campaigns 


\section{Appendix 2}

Online Web Survey Report

This section presents the quantitative data from the online web survey, with an interpretative outline indicating the frequencies of occurrences of the factors addressed in the survey questions.

\section{Question 1: What is your gender?}

\begin{tabular}{|ll|r|r|r|r|}
\hline & & Frequency & Percent & $\begin{array}{c}\text { Valid } \\
\text { Percent }\end{array}$ & $\begin{array}{c}\text { Cumulative } \\
\text { Percent }\end{array}$ \\
\hline Valid & Male & 5 & 10.6 & 10.9 & 10.9 \\
& Female & 41 & 87.2 & 89.1 & 100.0 \\
& Total & 46 & 97.9 & 100.0 & \\
Missing & System & 1 & 2.1 & & \\
Total & & 47 & 100.0 & & \\
\hline
\end{tabular}

\section{Table 2: Survey participants' gender}

Table 2 shows that $10.6 \%$ of the sample was male, $87.2 \%$ was female while $2.1 \%$ did not state their gender. Having five males among the 47 respondents of the survey is significant considering that WPc appears to be primarily targeted at women. The valid percentage in this table only accounts for those who responded to the question; it does not account for the one individual who did not declare gender. 


\begin{tabular}{|c|c|c|c|c|c|}
\hline & & Frequency & Percent & $\begin{array}{c}\text { Valid } \\
\text { Percent }\end{array}$ & $\begin{array}{c}\text { Cumulative } \\
\text { Percent }\end{array}$ \\
\hline \multirow[t]{4}{*}{ Valid } & $18-30$ & 17 & 36.2 & 36.2 & 36.2 \\
\hline & $31-40$ & 11 & 23.4 & 23.4 & 59.6 \\
\hline & $41+$ & 19 & 40.4 & 40.4 & 100.0 \\
\hline & Total & 47 & 100.0 & 100.0 & \\
\hline
\end{tabular}

Table 3: Survey participants' age

Table 3 shows that $36.2 \%$ of the sample was aged between 18 and 30, 23.4\% between 31 and 40 , while $40.4 \%$ was 41 years of age or older. This indicates that the majority of participants on WPc are in the age bracket of $18-30$ and $41+$, the latter slightly more than the former age group. 
Question 3: What is your marital status

\begin{tabular}{|c|c|c|c|c|c|}
\hline & & Frequency & Percent & $\begin{array}{l}\text { Valid } \\
\text { Percent }\end{array}$ & $\begin{array}{c}\text { Cumulative } \\
\text { Percent }\end{array}$ \\
\hline \multirow[t]{5}{*}{ Valid } & Single & 24 & 51.1 & 51.1 & 51.1 \\
\hline & Married & 17 & 36.2 & 36.2 & 87.2 \\
\hline & $\begin{array}{l}\text { Common } \\
\text { law }\end{array}$ & 4 & 8.5 & 8.5 & 95.7 \\
\hline & Divorced & 2 & 4.3 & 4.3 & 100.0 \\
\hline & Total & 47 & 100.0 & 100.0 & \\
\hline
\end{tabular}

Table 4: Survey participants' marital status

Table 4 shows that $51.1 \%$ of the sample was single, $36.2 \%$ married, $8.5 \%$ was in a common law relationship, while $4.3 \%$ was divorced. More than half of the participants are single. 
Question 4: Which region of the world do you live in?

\begin{tabular}{|c|c|c|c|c|c|c|}
\hline & & Frequency & Percent & $\begin{array}{c}\text { Valid } \\
\text { Percent }\end{array}$ & $\begin{array}{c}\text { Cumulative } \\
\text { Percent }\end{array}$ & Countries \\
\hline \multirow[t]{8}{*}{ Valid } & Africa & 20 & 42.6 & 42.6 & 42.6 & $\begin{array}{l}\text { Cameroon (1) } \\
\text { DR Congo (3) } \\
\text { Kenya (1) } \\
\text { Liberia (1) } \\
\text { Nigeria (10) } \\
\text { South Africa (1) } \\
\text { Tunisia (1) } \\
\text { Uganda (2) }\end{array}$ \\
\hline & Asia & 7 & 14.9 & 14.9 & 57.4 & $\begin{array}{l}\text { Kashmir (1) } \\
\text { Lebanon (1) } \\
\text { Myanmar (1) } \\
\text { Philippines (3) } \\
\text { Saudi Arabia (1) }\end{array}$ \\
\hline & Australia & 1 & 2.1 & 2.1 & 59.6 & Australia (1) \\
\hline & Europe & 4 & 8.5 & 8.5 & 68.1 & $\begin{array}{l}\text { Austria (1) } \\
\text { Netherlands (1) } \\
\text { Romania (1) } \\
\text { United Kingdom } \\
(1)\end{array}$ \\
\hline & $\begin{array}{l}\text { North } \\
\text { America }\end{array}$ & 13 & 27.7 & 27.7 & 95.7 & \begin{tabular}{|l} 
Canada (1) \\
United States of \\
America (12) \\
\end{tabular} \\
\hline & Oceania & 1 & 2.1 & 2.1 & 97.9 & New Zealand (1) \\
\hline & Undeclared & 1 & 2.1 & 2.1 & 100.0 & Undeclared (1) \\
\hline & Total & 47 & 100.0 & 100.0 & & \\
\hline
\end{tabular}

Table 5: Survey participants' location

Table 5 shows that $42.6 \%$ of the participants were from Africa, $14.9 \%$ Asia, 2.1\% Australia, 8.5\% Europe, 27.7\% North America, and 2.1\% Oceania; and 2.1\% did not state their 
location. Almost half of the respondents (20 out of 47) were from Africa with 10 out of that number located in Nigeria. More than a quarter of the respondents (13 out of 47) were based in North America, with 12 out of that number located in the United States of America. The third largest group (7 out of the 47) was from Asia, with the Philippines accounting for three out of those seven respondents.

Question 5: What is your highest level of education?

\begin{tabular}{|c|c|c|c|c|c|}
\hline & & Frequency & Percent & $\begin{array}{c}\text { Valid } \\
\text { Percent }\end{array}$ & $\begin{array}{c}\text { Cumulative } \\
\text { Percent }\end{array}$ \\
\hline \multirow[t]{6}{*}{ Valid } & Bachelors & 19 & 40.4 & 40.4 & 40.4 \\
\hline & Postgrad & 24 & 51.1 & 51.1 & 91.5 \\
\hline & Secondary & 1 & 2.1 & 2.1 & 93.6 \\
\hline & Trades & 2 & 4.3 & 4.3 & 97.9 \\
\hline & Undeclared & 1 & 2.1 & 2.1 & 100.0 \\
\hline & Total & 47 & 100.0 & 100.0 & \\
\hline
\end{tabular}

\section{Table 6: Survey participants' level of education}

Table 6 shows that $91.5 \%$ of the participants had a postsecondary level of education, $4.3 \%$ had trades certification, $2.1 \%$ had a secondary level of education, while $2.1 \%$ was undeclared. A significant percentage (51.5\% or 24 out of the 47 respondents) had a postgraduate level of education. 


\begin{tabular}{|c|c|c|c|c|c|}
\hline & & Frequency & Percent & $\begin{array}{c}\text { Valid } \\
\text { Percent }\end{array}$ & $\begin{array}{c}\text { Cumulative } \\
\text { Percent }\end{array}$ \\
\hline \multirow[t]{6}{*}{ Valid } & Paid & 41 & 87.2 & 87.2 & 87.2 \\
\hline & Retired & 1 & 2.1 & 2.1 & 89.4 \\
\hline & Undeclared & 1 & 2.1 & 2.1 & 91.5 \\
\hline & Unemployed & 1 & 2.1 & 2.1 & 93.6 \\
\hline & Volunteer & 3 & 6.4 & 6.4 & 100.0 \\
\hline & Total & 47 & 100.0 & 100.0 & \\
\hline
\end{tabular}

\section{Table 7: Survey participants' occupation/profession}

Table 7 shows that $87.2 \%$ of the participants were in paid employment, while $6.4 \%$ were in volunteer roles. The fact that $87.2 \%$ (accounting for 41 out of the 47 respondents) were in paid employment is significant, especially considering the amount of time they contribute to the campaigns on WPc. 


\begin{tabular}{|c|c|c|c|c|c|}
\hline & $\mathrm{N}$ & Minimum & Maximum & Mean & Std. Deviation \\
\hline $\begin{array}{l}\text { How much do you } \\
\text { earn per year? }\end{array}$ & 35 & 0 & 185000 & 26804.57 & 33984.923 \\
\hline Valid N (listwise) & 35 & & & & \\
\hline
\end{tabular}

\section{Table 8a: Survey participants' income}

Table 8 shows that for those individuals in paid employment, the mean income was USD $\$ 26,804.57(\mathrm{~s}=33,984.92)$. This is quite a large standard deviation that is possibly attributable to the fact that people would not give their exact income, but instead rounded it out. 


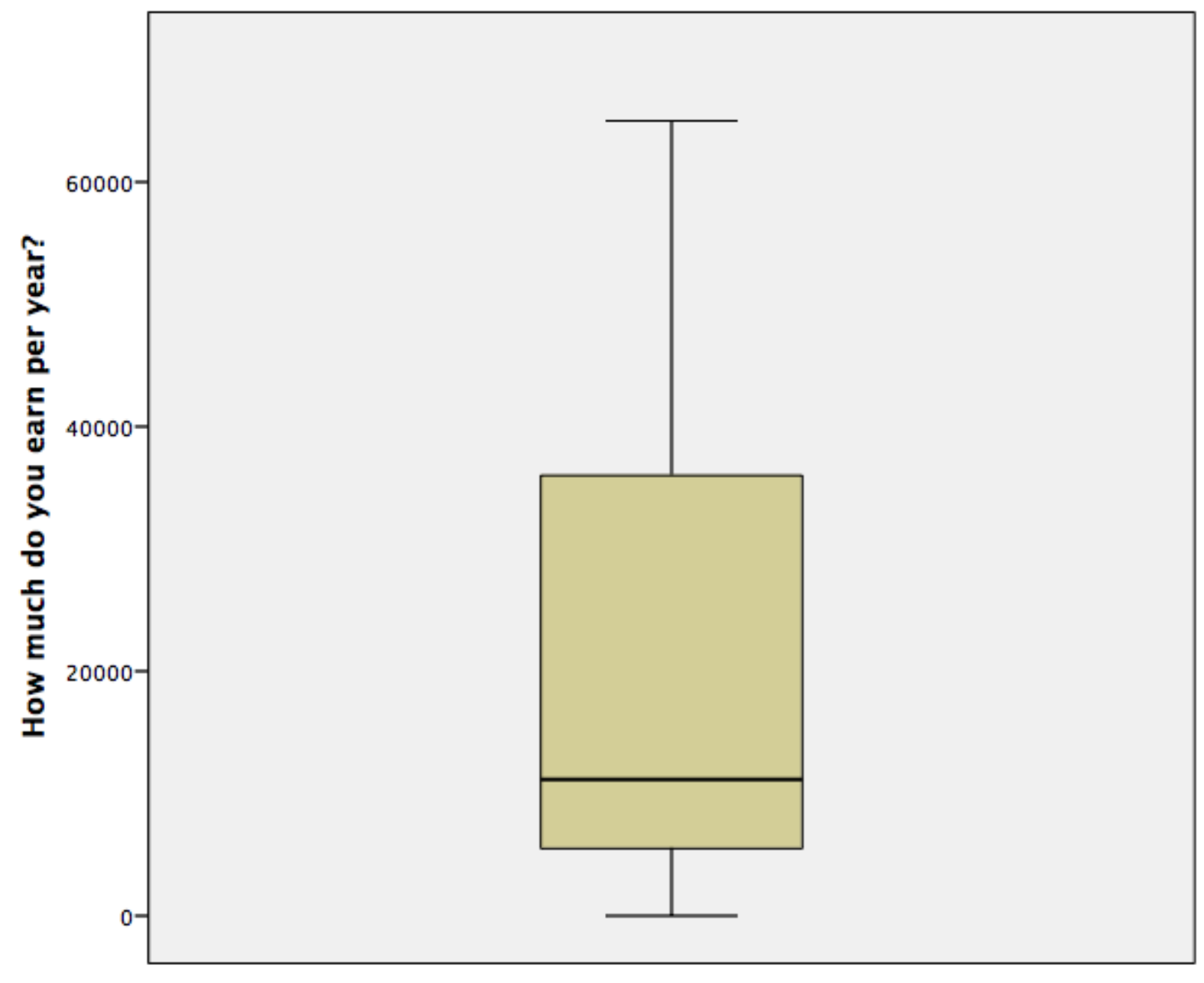

\section{Report}

How much do you earn per year?

\begin{tabular}{|c|r|c|l|}
\hline Mean & N & $\begin{array}{c}\text { Std. } \\
\text { Deviation }\end{array}$ & Median \\
\hline 22151.76 & 34 & 20231.466 & 11150.00 \\
\hline
\end{tabular}

Table 8b: Survey participants' income, after excluding those earning more than US\$100,000 per year. 
After excluding those earning more than US\$100,000 the bottom 50\% earn less than

US\$20,000/annum. The median is US\$11,150 among the whole group. One participant indicated an annual income of US\$185,000.

Question 8: Why did you join WorldPulse.com?

\begin{tabular}{|c|c|c|c|c|}
\hline & Frequency & Percent & $\begin{array}{c}\text { Valid } \\
\text { Percent }\end{array}$ & $\begin{array}{l}\text { Cumul } \\
\text { ative } \\
\text { Percent }\end{array}$ \\
\hline No responses & 15 & 31.9 & 31.9 & 31.9 \\
\hline 2005 & 1 & 2.1 & 2.1 & 34.0 \\
\hline $\begin{array}{l}\text { A passion for social justice, especially } \\
\text { where women are concerned }\end{array}$ & 1 & 2.1 & 2.1 & 36.2 \\
\hline $\begin{array}{l}\text { Because it is an interesting site to learn } \\
\text { about issues for women around the world, } \\
\text { to interact with other people, and hopefully } \\
\text { to be able to help some of them }\end{array}$ & 1 & 2.1 & 2.1 & 38.3 \\
\hline I am interested in women's rights & 1 & 2.1 & 2.1 & 40.4 \\
\hline $\begin{array}{l}\text { I joined as a Voices of Our Future mentor } \\
\text { because I wanted to help women who were } \\
\text { learning to write about their own } \\
\text { experiences and to support the inclusion of } \\
\text { women's voices in international dialogue. }\end{array}$ & 1 & 2.1 & 2.1 & 42.6 \\
\hline $\begin{array}{l}\text { I want to connect with women around the } \\
\text { world and sustain a global community }\end{array}$ & 1 & 2.1 & 2.1 & 44.7 \\
\hline $\begin{array}{l}\text { I want to write giving voice to marginalized } \\
\text { Filipino women }\end{array}$ & 1 & 2.1 & 2.1 & 46.8 \\
\hline $\begin{array}{l}\text { I wanted support and to share some of the } \\
\text { challenges I was facing. }\end{array}$ & 1 & 2.1 & 2.1 & 48.9 \\
\hline $\begin{array}{l}\text { I was searching for connections with } \\
\text { women on women and development issues }\end{array}$ & 1 & 2.1 & 2.1 & 51.1 \\
\hline $\begin{array}{l}\text { It was an opportunity for me to be a citizen } \\
\text { journalist and to magnify my voice on the } \\
\text { globe. It was also an opportunity for me to } \\
\text { interact, learn and share for the common } \\
\text { good of the society. }\end{array}$ & 1 & 2.1 & 2.1 & 53.2 \\
\hline
\end{tabular}




\begin{tabular}{|l|r|r|r|r|}
\hline Networking & 1 & 2.1 & 2.1 & 55.3 \\
\hline $\begin{array}{l}\text { Pour échanger les expériences avec les } \\
\text { femmes du monde entier }\end{array}$ & 1 & 2.1 & 2.1 & 57.4 \\
\hline Promote minority rights in Uganda. & 1 & 2.1 & 2.1 & 59.6 \\
\hline Searching & 1 & 2.1 & 2.1 & 61.7 \\
\hline $\begin{array}{l}\text { To advocate and empower women and help } \\
\text { raise our voices on issues pertinent to } \\
\text { women in particular but all people in } \\
\text { general. }\end{array}$ & 1 & 2.1 & 2.1 & 63.8 \\
\hline To be heard and to listen to others & 1 & 2.1 & 2.1 & 66.0 \\
\hline $\begin{array}{l}\text { To belong to a community of women round } \\
\text { the world }\end{array}$ & 1 & 2.1 & 2.1 & 68.1 \\
\hline To connect with women at the global level & 1 & 2.1 & 2.1 & 70.2 \\
\hline $\begin{array}{l}\text { To connect with like minded women the } \\
\text { world over }\end{array}$ & 1 & 2.1 & 2.1 & 72.3 \\
\hline To connect with other women & 1 & 2.1 & 2.1 & 74.5 \\
\hline $\begin{array}{l}\text { To connect with women in different parts } \\
\text { of the world }\end{array}$ & 1 & 2.1 & 2.1 & 76.6 \\
\hline To connect with women on global issues & 1 & 2.1 & 2.1 & 78.7 \\
\hline $\begin{array}{l}\text { To give something back to the world that } \\
\text { has given me so much! }\end{array}$ & 1 & 2.1 & 2.1 & 80.9 \\
\hline To help Congolese women exchange & 1 & 2.1 & 2.1 & 83.0 \\
\hline $\begin{array}{l}\text { To join my voice to that of the million of } \\
\text { women contributing to building a better } \\
\text { world. }\end{array}$ & 1 & 2.1 & 2.1 & 85.1 \\
\hline To learn & 1 & 2.1 & 2.1 & 91.5 \\
\hline $\begin{array}{l}\text { To learn more about the challenges and } \\
\text { solutions about women around the world }\end{array}$ & 1 & 2.1 & 2.1 & 87.2 \\
\hline $\begin{array}{l}\text { To meet others, read about issues that I am } \\
\text { interested in, hear other people's voices, } \\
\text { and just connect. }\end{array}$ & 1 & 2.1 & 2.1 & 89.4 \\
\hline $\begin{array}{l}\text { To share my views/ideas and discuss with } \\
\text { people }\end{array}$ & 1 & 2.1 & 2.1 & 100.0 \\
\hline $\begin{array}{l}\text { To study journalism and to contact with } \\
\text { women and women supporters }\end{array}$ & 1 & 2.1 & 2.1 & 93.6 \\
\hline To Volunteer within the Community & 100.0 & 97.7 \\
\hline $\begin{array}{l}\text { Why did I join? I can't answer. But why did } \\
\text { I continue? I can answer! }\end{array}$ & 1 & & 2.1 & \\
\hline Total & 1 & 1 & & \\
\hline
\end{tabular}


Table 9: All survey respondents indicated reasons why they joined WorldPulse.com

A recurring reason among the survey participants is that most of them joined because they wanted to connect with other women who are addressing issues pertaining to women in the world.

Question 9: How many hours a week do you spend on WorldPulse.com?

\begin{tabular}{|ll|r|r|r|r|}
\hline & & Frequency & Percent & $\begin{array}{c}\text { Valid } \\
\text { Percent }\end{array}$ & $\begin{array}{c}\text { Cumulative } \\
\text { Percent }\end{array}$ \\
\hline Valid & 1 & 16 & 34.0 & 37.2 & 37.2 \\
& $1-2$ & 12 & 25.5 & 27.9 & 65.1 \\
& $3+$ & 15 & 31.9 & 34.9 & 100.0 \\
& Total & 43 & 91.5 & 100.0 & \\
Missing & System & 4 & 8.5 & & \\
Total & & 47 & 100.0 & & \\
\hline
\end{tabular}

\section{Table 10: Time survey participants spend on WorldPulse.com}

Table 10 shows that $37.2 \%$ of the participants spent up to one hour per week on WPc, $27.9 \%$ spent $1-2$ hours, while $34.9 \%$ spent three or more hours per week. 


\begin{tabular}{|l|l|r|r|r|r|}
\hline \multicolumn{2}{|c}{ Question 10: How did you hear about WorldPulse.com? } \\
\hline \multirow{2}{*}{ Valid } & Frequency & Percent & $\begin{array}{c}\text { Valid } \\
\text { Percent }\end{array}$ & $\begin{array}{c}\text { Cumulative } \\
\text { Percent }\end{array}$ \\
\cline { 2 - 6 } & $\begin{array}{l}\text { Through my friend } \\
\text { Through a family }\end{array}$ & 5 & 10.6 & 11.6 & 11.6 \\
\cline { 2 - 7 } & $\begin{array}{l}\text { Through work or } \\
\text { business } \\
\text { association }\end{array}$ & 3 & 6.4 & 7.0 & 18.6 \\
\cline { 2 - 7 } & $\begin{array}{l}\text { Through online } \\
\text { search engine }\end{array}$ & 9 & 19.1 & 20.9 & 39.5 \\
\cline { 2 - 7 } & Other sources & 17 & 36.2 & 39.5 & 79.1 \\
\cline { 2 - 7 } & Total & 9 & 19.1 & 20.9 & 100.0 \\
\hline Missing & No response & 43 & 91.5 & 100.0 & \\
\hline Total & & 4 & 8.5 & & \\
\hline
\end{tabular}

Table 11a: Source of survey participants' referral to WorldPulse.com

Table 11 a shows that $11.6 \%$ of the participants had heard of WPc through a friend, $7 \%$ through a family member, $20.9 \%$ through a work or business association, $39.5 \%$ through online search engines, while $20.9 \%$ found out through other sources. 
Question 10: How did you hear about WorldPulse.com? - Other sources

\begin{tabular}{|l|r|r|r|r|}
\hline & Frequency & Percent & $\begin{array}{c}\text { Valid } \\
\text { Percent }\end{array}$ & $\begin{array}{c}\text { Cumulative } \\
\text { Percent }\end{array}$ \\
\hline No response & 38 & 80.9 & 80.9 & 80.9 \\
\hline Through a webpage & 1 & 2.1 & 2.1 & 83.0 \\
\hline Facebook post & 1 & 2.1 & 2.1 & 85.1 \\
\hline From the book Half the Sky & 1 & 2.1 & 2.1 & 87.2 \\
\hline $\begin{array}{l}\text { Through an ad for the print } \\
\text { magazine (which I later } \\
\text { purchased) in another magazine }\end{array}$ & 1 & 2.1 & 2.1 & 89.4 \\
\hline LinkedIn & 1 & 2.1 & 2.1 & 91.5 \\
\hline Online feminist networks & 1 & 2.1 & 2.1 & 93.6 \\
\hline $\begin{array}{l}\text { Through an article on a website } \\
\text { that mentioned it }\end{array}$ & 1 & 2.1 & 2.1 & 95.7 \\
\hline Through my email & 1 & 2.1 & 2.1 & 97.9 \\
\hline $\begin{array}{l}\text { Through my trainer from Salus } \\
\text { World }\end{array}$ & 1 & 2.1 & 2.1 & 100.0 \\
\hline Total & 47 & 100.0 & 100.0 & \\
\hline
\end{tabular}

Table 11b: Other sources of survey participants' referral to WorldPulse.com

Table $11 \mathrm{~b}$ shows that $80.9 \%$ did not indicate their source of referral to WPc while some of the survey respondents indicated they were referred to WorldPulse.com through other platforms and individual referrers. 
Question 11: When did you join WorldPulse.com?

\begin{tabular}{|c|r|r|r|r|}
\hline & & & $\begin{array}{c}\text { Valid } \\
\text { Percent }\end{array}$ & $\begin{array}{c}\text { Cumulative } \\
\text { Percent }\end{array}$ \\
\hline No response & 7 & 14.9 & 14.9 & 14.9 \\
2005 & 1 & 2.1 & 2.1 & 17.0 \\
2009 & 9 & 19.1 & 19.1 & 36.2 \\
2010 & 5 & 10.6 & 10.6 & 46.8 \\
2011 & 4 & 8.5 & 8.5 & 55.3 \\
2012 & 3 & 6.4 & 6.4 & 61.7 \\
2013 & 7 & 14.9 & 14.9 & 76.6 \\
2014 & 7 & 14.9 & 14.9 & 91.5 \\
2015 & 4 & 8.5 & 8.5 & 100.0 \\
Total & 47 & 100.0 & 100.0 & \\
\hline
\end{tabular}

\section{Table 12: When survey participants joined WorldPulse.com}

Table 12 shows that $14.9 \%$ of the respondents did not state when they joined the website, $2.1 \%$ joined in $2005,19.1 \%$ in $2009,10.6 \%$ in $2010,8.5 \%$ in $2011,6.4 \%$ in $2012,14.9 \%$ each in 2013 and 2014, and 8.5\% in 2015. A significant proportion - 36\% of the survey respondents, or 17 out of 47 - joined WPc between 2012 and 2014 (during the three digital action campaigns: 'Ending Gender-Based Violence 2012', 'Girls Transform The World 2013' and 'Women Weave the Web 2014'). An even more significant proportion $74 \%$ of the survey respondents, or 35 out of 47 - joined WPc between 2009 and 2014 (during the four sessions of the 'Voices of Our Future' citizen journalism training program). 
Question 12: Are you a member of any of the sub-committees on WorldPulse.com?

\begin{tabular}{|c|c|c|c|c|c|}
\hline & & Frequency & Percent & $\begin{array}{c}\text { Valid } \\
\text { Percent }\end{array}$ & $\begin{array}{c}\text { Cumulative } \\
\text { Percent }\end{array}$ \\
\hline & Yes & 24 & 51.1 & 57.1 & 57.1 \\
\hline & No & 18 & 38.3 & 42.9 & 100.0 \\
\hline & Total & 42 & 89.4 & 100.0 & \\
\hline Missing & No & 5 & 106 & & \\
\hline & response & 3 & 10.0 & & \\
\hline Total & & 47 & 100.0 & & \\
\hline
\end{tabular}

Table 13a: Survey participants' sub-committee memberships on WorldPulse.com

Table 13a shows that $51.1 \%$ of the respondents were sub-committee members on WPc, $38.3 \%$ were not sub-committee members, while $10.6 \%$ did not provide this information. After omitting the missing data for the undeclared information, the valid percent indicates that $57.1 \%$ of the respondents are possibly sub-committee members while $42.9 \%$ are probably not. 


\begin{tabular}{|c|c|c|c|c|}
\hline \multicolumn{5}{|c|}{$\begin{array}{l}\text { Question 12: Are you a member of any of the sub-committees on } \\
\text { WorldPulse.com? }\end{array}$} \\
\hline If yes, which committee? & $\begin{array}{c}\text { Frequen } \\
\text { cy }\end{array}$ & Percent & $\begin{array}{c}\text { Valid } \\
\text { Percent }\end{array}$ & $\begin{array}{l}\text { Cumulat } \\
\text { ive } \\
\text { Percent } \\
\end{array}$ \\
\hline No response & 14 & 29.8 & 29.8 & 29.8 \\
\hline Advisory Board member & 1 & 2.1 & 2.1 & 31.9 \\
\hline Can't remember & 1 & 2.1 & 2.1 & 34.0 \\
\hline Economic Empowerment & 1 & 2.1 & 2.1 & 36.2 \\
\hline Haven't taken the time to sign up yet & 1 & 2.1 & 2.1 & 38.3 \\
\hline I do not understand which sub committees & 1 & 2.1 & 2.1 & 40.4 \\
\hline $\begin{array}{l}\text { I have served as an Editorial Midwife in } \\
\text { the past on World Pulse. However, I never } \\
\text { did serve on any Sub-Committee. }\end{array}$ & 1 & 2.1 & 2.1 & 42.6 \\
\hline $\begin{array}{l}\text { I'm okay just being online with other } \\
\text { women }\end{array}$ & 1 & 2.1 & 2.1 & 44.7 \\
\hline Je ne suis pas informée & 1 & 2.1 & 2.1 & 46.8 \\
\hline Just joined. Looking around & 1 & 2.1 & 2.1 & 48.9 \\
\hline Lack of time & 1 & 2.1 & 2.1 & 51.1 \\
\hline Leadership & 1 & 2.1 & 2.1 & 53.2 \\
\hline $\begin{array}{l}\text { Leadership and Economic Empowerment } \\
\text { group }\end{array}$ & 1 & 2.1 & 2.1 & 55.3 \\
\hline Leadership and Empowerment & 1 & 2.1 & 2.1 & 57.4 \\
\hline
\end{tabular}




\begin{tabular}{|c|c|c|c|c|}
\hline Leadership Group & 1 & 2.1 & 2.1 & 59.6 \\
\hline Leadership, Economic Empowerment & 1 & 2.1 & 2.1 & 61.7 \\
\hline Leadership/VOF & 1 & 2.1 & 2.1 & 63.8 \\
\hline Less time & 1 & 2.1 & 2.1 & 66.0 \\
\hline Listener, Contributors, VOF 2012 & 1 & 2.1 & 2.1 & 68.1 \\
\hline Mostly Teaching WP, not posting & 1 & 2.1 & 2.1 & 70.2 \\
\hline New member & 1 & 2.1 & 2.1 & 72.3 \\
\hline Nigeria cafe & 1 & 2.1 & 2.1 & 74.5 \\
\hline Not had time & 1 & 2.1 & 2.1 & 76.6 \\
\hline Not involved & 1 & 2.1 & 2.1 & 78.7 \\
\hline Only as a listener & 1 & 2.1 & 2.1 & 80.9 \\
\hline VOF & 1 & 2.1 & 2.1 & 83.0 \\
\hline VOF 2009 & 1 & 2.1 & 2.1 & 85.1 \\
\hline VOF Alumni & 1 & 2.1 & 2.1 & 87.2 \\
\hline VOF Alumni & 1 & 2.1 & 2.1 & 89.4 \\
\hline Volunteer & 1 & 2.1 & 2.1 & 91.5 \\
\hline $\begin{array}{l}\text { Welcome Committee, GBV Hub Page } \\
\text { Management Committee, Online } \\
\text { Community Champion-Leadership Group, } \\
\text { Voices of Our Future }\end{array}$ & 1 & 2.1 & 2.1 & 93.6 \\
\hline $\begin{array}{l}\text { Welcomer, Listener, Contributor, } \\
\text { Leadership Group, Economics Group }\end{array}$ & 1 & 2.1 & 2.1 & 95.7 \\
\hline Welcomers and Listeners & 1 & 2.1 & 2.1 & 97.9 \\
\hline World Pulse Volunteer Group & 1 & 2.1 & 2.1 & 100.0 \\
\hline Total & 47 & 100.0 & 100.0 & \\
\hline
\end{tabular}

Table 13b: Survey participants' sub-committee memberships on WorldPulse.com

Table $13 \mathrm{~b}$ shows that out of the $51.1 \%$ survey respondents who are sub-committee members some of them belong to multiple sub-committees, including six respondents who are part of the 'Voices of Our Future' training group. 
Question 13: Which of the following information would you

like to know about other participants when engaging on

WorldPulse.com - Race

\begin{tabular}{|l|r|r|r|c|}
\hline & Frequency & Percent & Palid & Percent \\
Percent \\
\hline Valid 1
\end{tabular}

Table 14a: Information survey participants would like to know - Race

Table 14 a shows that $29.3 \%$ of the respondents would have liked to know the race of other participants, while $70.7 \%$ did not.

Question 13: Which of the following information would you

like to know about other participants when engaging on

WorldPulse.com - Gender

\begin{tabular}{|l|r|r|r|c|}
\hline & Frequency & Percent & Percent & $\begin{array}{c}\text { Cumulative } \\
\text { Percent }\end{array}$ \\
\hline Valid 1 & 22 & 53.7 & 100.0 & 100.0 \\
Missing System & 19 & 46.3 & & \\
Total & 41 & 100.0 & & \\
\hline
\end{tabular}

Table 14b: Information survey participants would like to know - Gender

Table $14 \mathrm{~b}$ shows that $53.7 \%$ of the respondents would have liked to know the gender of other participants, while $46.3 \%$ did not. 
Question 13: Which of the following information would you

like to know about other participants when engaging on

WorldPulse.com - Age

\begin{tabular}{|l|r|r|r|c|}
\hline & Frequency & Percent & $\begin{array}{c}\text { Valid } \\
\text { Percent }\end{array}$ & $\begin{array}{c}\text { Cumulative } \\
\text { Percent }\end{array}$ \\
\hline Valid 1 & 11 & 26.8 & 100.0 & 100.0 \\
Missing System & 30 & 73.2 & & \\
Total & 41 & 100.0 & & \\
\hline
\end{tabular}

Table 14c: Information survey participants would like to know - Age

Table $14 \mathrm{c}$ shows that $26.8 \%$ of the respondents wanted to know the age of other participants, while $73.2 \%$ did not.

Question 13: Which of the following information would you like to know about other participants when engaging on WorldPulse.com - Profession

\begin{tabular}{|l|r|r|c|c|}
\hline & Frequency & Percent & $\begin{array}{c}\text { Valid } \\
\text { Percent }\end{array}$ & $\begin{array}{c}\text { Cumulative } \\
\text { Percent }\end{array}$ \\
\hline Valid 1 & 26 & 63.4 & 100.0 & 100.0 \\
Missing System & 15 & 36.6 & & \\
Total & 41 & 100.0 & & \\
\hline
\end{tabular}

Table 14d: Information survey participants would like to know - Profession

Table $14 \mathrm{~d}$ shows that $63.4 \%$ of the respondents wanted to know the profession of other participants, while $36.6 \%$ did not. 
Question 13: Which of the following information would you

like to know about other participants when engaging on

WorldPulse.com - Level of education

\begin{tabular}{|l|r|r|r|r|r|r|}
\hline & & Frequency & Percent & \multicolumn{1}{|l|}{$\begin{array}{c}\text { Valid } \\
\text { Percent }\end{array}$} & $\begin{array}{c}\text { Cumulative } \\
\text { Percent }\end{array}$ \\
\hline lid 1 & 13 & 31.7 & 100.0 & 100.0 & \\
ssing System & 28 & 68.3 & & & & Va \\
al & 41 & 100.0 & & & & Mi \\
To1
\end{tabular}

le 14e: Information survey participants would like to know - Education

Tab

le $14 \mathrm{e}$ shows that $31.7 \%$ of the respondents wanted to know the education level of $\mathrm{Tab}$ r participants, while $68.3 \%$ did not.

othe 
Question 14: Have you participated in any of the Digital Action Campaigns on WorldPulse.com?

\begin{tabular}{|ll|r|r|r|r|}
\hline & & Frequency & Percent & Valid & Percent \\
Valid & Yes & 17 & 36.2 & 58.6 & 58.6 \\
& No & 12 & 25.5 & 41.4 & 100.0 \\
& Total & 29 & 61.7 & 100.0 & \\
Missing & System & 18 & 38.3 & & \\
Total & & 47 & 100.0 & & \\
\hline
\end{tabular}

\section{Table 15a: Participation in Digital Action Campaigns}

Table $15 \mathrm{a}$ shows that $36.2 \%$ of the respondents had participated in the Digital Action Campaigns, 25.5\% had not participated in the Digital Action Campaigns, while 38.3\% did not provide this information. When the undeclared is excluded $58.6 \%$ - more than half of those declaring this information - have participated in the Digital Action Campaigns on WorldPulse.com. 


\begin{tabular}{|c|c|c|c|c|}
\hline \multicolumn{5}{|c|}{$\begin{array}{c}\text { Question 14: Have you participated in any of the Digital Action } \\
\text { Campaigns on WorldPulse.com? - TEXT }\end{array}$} \\
\hline Yes, why? No, why not? & Frequency & Percent & $\begin{array}{c}\text { Valid } \\
\text { Percent }\end{array}$ & $\begin{array}{l}\text { Cumulative } \\
\text { Percent }\end{array}$ \\
\hline No response & 19 & 40.4 & 40.4 & 40.4 \\
\hline 2012 & 1 & 2.1 & 2.1 & 42.6 \\
\hline All & 1 & 2.1 & 2.1 & 44.7 \\
\hline Busy & 1 & 2.1 & 2.1 & 46.8 \\
\hline Busy with work & 1 & 2.1 & 2.1 & 48.9 \\
\hline Did not have time & 1 & 2.1 & 2.1 & 51.1 \\
\hline $\begin{array}{l}\text { Digital Action Campaign to } \\
\text { Stop Violence Against Women, } \\
\text { Girls Transform the World, } \\
\text { Women Weave the Web }\end{array}$ & 1 & 2.1 & 2.1 & 53.2 \\
\hline $\begin{array}{l}\text { Ending Violence Against } \\
\text { Women and Women Weave the } \\
\text { Web }\end{array}$ & 1 & 2.1 & 2.1 & 55.3 \\
\hline $\begin{array}{l}\text { Girls Transform the World and } \\
\text { Women Weave the Web }\end{array}$ & 1 & 2.1 & 2.1 & 57.4 \\
\hline Haven't gotten around to it yet. & 1 & 2.1 & 2.1 & 59.6 \\
\hline I am new to the platform & 1 & 2.1 & 2.1 & 61.7 \\
\hline I didn't know about it & 1 & 2.1 & 2.1 & 63.8 \\
\hline Just joined & 1 & 2.1 & 2.1 & 66.0 \\
\hline Manque de temps & 1 & 2.1 & 2.1 & 68.1 \\
\hline $\begin{array}{l}\text { More than three, forgotten the } \\
\text { themes. }\end{array}$ & 1 & 2.1 & 2.1 & 70.2 \\
\hline No & 1 & 2.1 & 2.1 & 72.3 \\
\hline No time to write long articles & 1 & 2.1 & 2.1 & 74.5 \\
\hline No & 1 & 2.1 & 2.1 & 76.6 \\
\hline
\end{tabular}




\begin{tabular}{|c|c|c|c|c|}
\hline $\begin{array}{l}\text { Rio+20: United Nations } \\
\text { Conference on Sustainable } \\
\text { Development, Voices of Our } \\
\text { Future } 2012 \text { and 2013, Singing } \\
\text { Her Legacy: Wangari Maathai } \\
\text { Tribute, Singing Her Legacy: } \\
\text { Wangari Maathai Tribute, } \\
\text { Maternal Health, Ending } \\
\text { Gender-Based Violence 2012, } \\
\text { Take Back the echnology, } \\
\text { Women Weave the Web, } \\
\text { BringBackOurGirls, }\end{array}$ & 1 & 2.1 & 2.1 & 78.7 \\
\hline Served as an Editorial Midwife. & 1 & 2.1 & 2.1 & 80.9 \\
\hline Technology for women & 1 & 2.1 & 2.1 & 83.0 \\
\hline $\begin{array}{l}\text { The WWW campaign as a } \\
\text { volunteer listener }\end{array}$ & 1 & 2.1 & 2.1 & 85.1 \\
\hline Voices of Future, 2012 & 1 & 2.1 & 2.1 & 87.2 \\
\hline Voices of Our Future Program & 1 & 2.1 & 2.1 & 89.4 \\
\hline Wasn't aware of WP & 1 & 2.1 & 2.1 & 91.5 \\
\hline $\begin{array}{l}\text { Women Weave the Web digital } \\
\text { campaign; Ending Violence } \\
\text { Against Women }\end{array}$ & 1 & 2.1 & 2.1 & 93.6 \\
\hline Women Weave the Web, 2014 & 1 & 2.1 & 2.1 & 95.7 \\
\hline WWW & 1 & 2.1 & 2.1 & 97.9 \\
\hline WWW, Education & 1 & 2.1 & 2.1 & 100.0 \\
\hline Total & 47 & 100.0 & 100.0 & \\
\hline
\end{tabular}

Table 15b: Participation in Digital Action Campaigns

Table $15 \mathrm{~b}$ shows that 16 survey respondents had participated in at least one of the Digital Action Campaigns while 12 respondents said either they did not have time to participate, they were not aware of the DACs or they had just joined the WPc platform. 
Question 15: Why did you participate in the Digital Action Campaigns on WorldPulse.com?

\begin{tabular}{|c|c|c|c|c|}
\hline & Frequency & Percent & $\begin{array}{c}\text { Valid } \\
\text { Percent }\end{array}$ & $\begin{array}{l}\text { Cumulati } \\
\text { ve } \\
\text { Percent }\end{array}$ \\
\hline $\begin{array}{l}\text { This was a campaign that I was } \\
\text { very interested in specifically } \\
\text { because I worked as a journalist } \\
\text { for many years focusing primarily } \\
\text { on women's issues in both the print } \\
\text { and electronic media. }\end{array}$ & 1 & 2.1 & 2.1 & 2.1 \\
\hline No response & 30 & 63.8 & 63.8 & 66.0 \\
\hline $\begin{array}{l}\text { Because I believe in the } \\
\text { synergistic power of digital } \\
\text { campaigns }\end{array}$ & 1 & 2.1 & 2.1 & 68.1 \\
\hline $\begin{array}{l}\text { Because I believed in the change } \\
\text { the campaigns stood for and } \\
\text { counted it important to make my } \\
\text { voice heard by making my } \\
\text { contributions }\end{array}$ & 1 & 2.1 & 2.1 & 70.2 \\
\hline $\begin{array}{l}\text { Because it seemed like an } \\
\text { interesting thing to volunteer for }\end{array}$ & 1 & 2.1 & 2.1 & 72.3 \\
\hline $\begin{array}{l}\text { For a global connection, in order } \\
\text { to learn from the experiences of } \\
\text { the women on various issues that } \\
\text { affect them on day to day life }\end{array}$ & 1 & 2.1 & 2.1 & 74.5 \\
\hline $\begin{array}{l}\text { For Fun! And we did have loads of } \\
\text { fun. }\end{array}$ & 1 & 2.1 & 2.1 & 76.6 \\
\hline $\begin{array}{l}\text { Glad to be able to find out about } \\
\text { and directly support women }\end{array}$ & 1 & 2.1 & 2.1 & 78.7 \\
\hline $\begin{array}{l}\text { I wanted the voices from Uganda } \\
\text { to be heard }\end{array}$ & 1 & 2.1 & 2.1 & 80.9 \\
\hline
\end{tabular}




\begin{tabular}{|c|c|c|c|c|}
\hline $\begin{array}{l}\text { It sharpened my voice and } \\
\text { improved my vision of myself and } \\
\text { the world around }\end{array}$ & 1 & 2.1 & 2.1 & 83.0 \\
\hline $\begin{array}{l}\text { It was a chance to share my unique } \\
\text { voice and solutions for change } \\
\text { with the global community }\end{array}$ & 1 & 2.1 & 2.1 & 85.1 \\
\hline Not applicable & 1 & 2.1 & 2.1 & 87.2 \\
\hline $\begin{array}{l}\text { To add my voice to the world } \\
\text { pulse global audience }\end{array}$ & 1 & 2.1 & 2.1 & 89.4 \\
\hline To advocate for women's right & 1 & 2.1 & 2.1 & 91.5 \\
\hline $\begin{array}{l}\text { To contribute my voice and the } \\
\text { voices of the voiceless women I } \\
\text { am working with in my } \\
\text { community }\end{array}$ & 1 & 2.1 & 2.1 & 93.6 \\
\hline $\begin{array}{l}\text { To contribute my voice to the } \\
\text { Campaign, to encourage others, to } \\
\text { add the Congolese voice to the } \\
\text { world's chorus, to tell of our } \\
\text { situation or tell of how we are } \\
\text { working toward the goal of the } \\
\text { Campaign }\end{array}$ & 1 & 2.1 & 2.1 & 95.7 \\
\hline $\begin{array}{l}\text { To encourage the women who } \\
\text { were writing about their } \\
\text { experiences. }\end{array}$ & 1 & 2.1 & 2.1 & 97.9 \\
\hline $\begin{array}{l}\text { To involve our rural women who } \\
\text { live in area without electricity. I } \\
\text { went with my laptop to the village. }\end{array}$ & 1 & 2.1 & 2.1 & 100.0 \\
\hline Total & 47 & 100.0 & 100.0 & \\
\hline
\end{tabular}

Table 16: Why survey respondents participated in the Digital Action Campaigns

Table 16 shows that 16 of the survey respondents indicated diverse reasons why they participated in the Digital Action Campaigns. One reason that stood out among these responses was the participants' interest in sharing their views on issues they are passionate 
about.

Question 17: Have you ever applied for the Voices of Our Future training on WorldPulse.com?

\begin{tabular}{|l|r|r|r|r|}
\hline & Frequency & Percent & $\begin{array}{c}\text { Valid } \\
\text { Percent }\end{array}$ & $\begin{array}{c}\text { Cumulative } \\
\text { Percent }\end{array}$ \\
\hline Yes & 23 & 48.9 & 59.0 & 59.0 \\
No & 16 & 34.0 & 41.0 & 100.0 \\
Total & 39 & 83.0 & 100.0 & \\
Missing System & 8 & 17.0 & & \\
Total & 47 & 100.0 & & \\
\hline
\end{tabular}

\section{Table 17: Applicants to the 'Voices of Our Future' citizen journalism training}

Table 17 shows that $48.9 \%$ of the respondents had applied to participate in the 'Voices of Our Future' training program, 34\% had not applied, while 17\% did not provide this information. This $48.9 \%$ accounts for 23 people, nearly half of the total 47 of those responding to the survey. When the undeclared is excluded, a significant $59 \%$ of the respondents have applied to the 'Voices of Our Future' training program. 
Question 18: Have you taken the Voices of Our Future training on WorldPulse.com?

\begin{tabular}{|ll|r|r|r|r|}
\hline & & Frequency & Percent & $\begin{array}{c}\text { Valid } \\
\text { Percent }\end{array}$ & $\begin{array}{c}\text { Cumulative } \\
\text { Percent }\end{array}$ \\
\hline Valid & Yes & 22 & 46.8 & 56.4 & 56.4 \\
& No & 17 & 36.2 & 43.6 & 100.0 \\
& Total & 39 & 83.0 & 100.0 & \\
Missing & System & 8 & 17.0 & & \\
Total & 47 & 100.0 & & \\
\hline
\end{tabular}

\section{Table 18a: Participants in the 'Voices of Our Future' citizen journalism training}

Table 18 shows that $46.8 \%$ of the respondents had participated in the 'Voices of Our Future' training program, $36.2 \%$ had not, and $17 \%$ did not provide this information. This $46.8 \%$ accounts for 22 out of the 23 people who had applied to the training, nearly half of the total 47 of those responding to the survey. When the undeclared is excluded, a significant $56.4 \%$ of the respondents have participated in the 'Voices of Our Future' training program. 


\begin{tabular}{|c|c|c|c|c|}
\hline \multicolumn{5}{|c|}{$\begin{array}{l}\text { Question 18: Have you taken the Voices of Our Future training on } \\
\text { WorldPulse.com? - TEXT }\end{array}$} \\
\hline If yes, why? If no, why not? & Frequency & Percent & $\begin{array}{c}\text { Valid } \\
\text { Percent } \\
\end{array}$ & $\begin{array}{l}\text { Cumulative } \\
\text { Percent }\end{array}$ \\
\hline No responses & 16 & 34.0 & 34.0 & 34.0 \\
\hline 2009 & 4 & 8.5 & 8.5 & 42.6 \\
\hline 2010 & 5 & 10.6 & 10.6 & 53.2 \\
\hline 2011 & 1 & 2.1 & 2.1 & 55.3 \\
\hline 2012 & 3 & 6.4 & 6.4 & 61.7 \\
\hline 2013 & 8 & 17.0 & 17.0 & 78.7 \\
\hline $\begin{array}{l}\text { I became very busy at } \\
\text { work, home, church and } \\
\text { community }\end{array}$ & 1 & 2.1 & 2.1 & 80.9 \\
\hline $\begin{array}{l}\text { I have courses I am taking } \\
\text { for now }\end{array}$ & 1 & 2.1 & 2.1 & 83.0 \\
\hline Just joined & 1 & 2.1 & 2.1 & 85.1 \\
\hline Lack of time & 1 & 2.1 & 2.1 & 87.2 \\
\hline Manque d'information & 1 & 2.1 & 2.1 & 89.4 \\
\hline No & 1 & 2.1 & 2.1 & 91.5 \\
\hline $\begin{array}{l}\text { No reason in particular. I } \\
\text { am not opposed to taking } \\
\text { part in it when I have the } \\
\text { time to fully commit to it. }\end{array}$ & 1 & 2.1 & 2.1 & 93.6 \\
\hline No time yet & 1 & 2.1 & 2.1 & 95.7 \\
\hline Not yet & 1 & 2.1 & 2.1 & 97.9 \\
\hline Yes 2013 & 1 & 2.1 & 2.1 & 100.0 \\
\hline Total & 47 & 100.0 & 100.0 & \\
\hline
\end{tabular}

Table 18b: Participants in the 'Voices of Our Future' citizen journalism training

Table $18 \mathrm{~b}$ shows that 22 of the 47 survey respondents indicated the years and why they took the 'Voices of Our Future' citizen journalism training program. 
Question 19: If you took the Voices of Our Future training, have you had any of your stories published in any media?

\begin{tabular}{|l|r|r|r|r|}
\hline & Frequency & Percent & $\begin{array}{c}\text { Valid } \\
\text { Percent }\end{array}$ & $\begin{array}{c}\text { Cumulative } \\
\text { Percent }\end{array}$ \\
\hline Yes & 19 & 40.4 & 70.4 & 70.4 \\
No & 8 & 17.0 & 29.6 & 100.0 \\
Total & 27 & 57.4 & 100.0 & \\
Missing System & 20 & 42.6 & & \\
Total & 47 & 100.0 & & \\
\hline
\end{tabular}

\section{Table 19a: Publishing after the 'Voices of Our Future' citizen journalism training}

Table $19 \mathrm{a}$ shows that $40.4 \%$ of the respondents who participated in the 'Voices of Our Future' training program had their stories published, 17\% did not get their stories published, while $20 \%$ did not provide this information. This $40.4 \%$ accounts for 19 people (out of the 23 that applied and the 22 that participated in the "Voices of Our Future' training program, and nearly half of the total 47 of those responding to the survey). When the undeclared is excluded, a significant $70.4 \%$ of the respondents have had their stories published after taking the 'Voices of Our Future' training program. 


\begin{tabular}{|c|c|c|c|c|}
\hline \multicolumn{5}{|c|}{$\begin{array}{c}\text { Question 19: If you took the Voices of Our Future training, have you had any of } \\
\text { your stories published in any media? - TEXT }\end{array}$} \\
\hline & Frequency & Percent & \begin{tabular}{c|} 
Valid \\
Percent \\
\end{tabular} & $\begin{array}{c}\text { Cumulative } \\
\text { Percent } \\
\end{array}$ \\
\hline No response & 31 & 66.0 & 66.0 & 66.0 \\
\hline Chime for change & 1 & 2.1 & 2.1 & 68.1 \\
\hline Chime for Change & 1 & 2.1 & 2.1 & 70.2 \\
\hline Global Press Institute & 1 & 2.1 & 2.1 & 72.3 \\
\hline Global Press Journal & 1 & 2.1 & 2.1 & 74.5 \\
\hline $\begin{array}{l}\text { Global Press Journal, Women } \\
\text { E-News }\end{array}$ & 1 & 2.1 & 2.1 & 76.6 \\
\hline GPI & 1 & 2.1 & 2.1 & 78.7 \\
\hline $\begin{array}{l}\text { Huffington Post, Catholic } \\
\text { Reporter, and quoted in } \\
\text { numerous publications }\end{array}$ & 1 & 2.1 & 2.1 & 80.9 \\
\hline Huffington Post & 1 & 2.1 & 2.1 & 83.0 \\
\hline Independent online media & 1 & 2.1 & 2.1 & 85.1 \\
\hline International & 1 & 2.1 & 2.1 & 87.2 \\
\hline $\begin{array}{l}\text { Mail and Guardian and local } \\
\text { newspapers }\end{array}$ & 1 & 2.1 & 2.1 & 89. \\
\hline $\begin{array}{l}\text { Mindanao Goldstar Daily, } \\
\text { Philippine Daily Inquirer }\end{array}$ & 1 & 2.1 & 2.1 & 91.5 \\
\hline Only in World Pulse & 1 & 2.1 & 2.1 & 93.6 \\
\hline Several Blogs and Websites & 1 & 2.1 & 2.1 & 95.7 \\
\hline $\begin{array}{l}\text { Violence is not our culture, } \\
\text { Margarita Caetano, Amazing } \\
\text { Women Rock, Safe World } \\
\text { Community, Women's UN } \\
\text { Report Network, Easy Bib } \\
\text { Research, The Heartland } \\
\text { Institute Newsletters, DARF, } \\
\text { Female Genital Mutilation, } \\
\text { IFRC, etc. }\end{array}$ & 1 & 2.1 & 2.1 & 97.9 \\
\hline Yes & 1 & 2.1 & 2.1 & 100.0 \\
\hline Total & 47 & 100.0 & 100.0 & \\
\hline
\end{tabular}

Table 19b: Publishing after the 'Voices of Our Future' citizen journalism training 
Table $19 \mathrm{~b}$ shows that 16 out of the 22 'Voices of Our Future' citizen journalism training participants had their stories published. This indicates that a significant $73 \%$ out of the total number of VOF participants responding to the survey have had their stories published either on WPc or through other media channels. Some of the participants have had publication on multiple platforms.

Questions 16 and 20 asked survey respondents to supply their information if they would like to be contacted for follow-up interviews. The answers to these two questions are omitted from this report to further protect the privacy of the survey respondents. 
Appendix 3

\section{Questions 4 and 14}

Which region of the world do you live in? * Have you participated in any of the Digital Action Campaigns on WorldPulse.com?

Count

\begin{tabular}{|c|c|c|c|}
\hline \multirow{2}{*}{$\begin{array}{l}\text { Which region of the world } \\
\text { do you live in? }\end{array}$} & \multicolumn{2}{|c|}{$\begin{array}{c}\text { Have you participated in any of the } \\
\text { Digital Action Campaigns on } \\
\text { WorldPulse.com? }\end{array}$} & \multirow[b]{2}{*}{ Total } \\
\hline & Yes & No & \\
\hline North America & 4 & 2 & 6 \\
\hline Africa & 7 & 7 & 14 \\
\hline Other & 6 & 2 & 8 \\
\hline Total & 17 & 11 & 28 \\
\hline
\end{tabular}

Table 20: Cross-tabulation of survey respondents' location and digital action campaign participation.

Chi-Square Tests

\begin{tabular}{|c|c|c|c|c|c|c|}
\hline & Value & $\mathrm{df}$ & $\begin{array}{c}\text { Asymp. Sig. } \\
\text { (2-sided) }\end{array}$ & $\begin{array}{c}\text { Exact Sig. } \\
\text { (2-sided) }\end{array}$ & $\begin{array}{c}\text { Exact Sig. } \\
\text { (1-sided) }\end{array}$ & $\begin{array}{c}\text { Point } \\
\text { Probability } \\
\end{array}$ \\
\hline $\begin{array}{l}\text { Pearson Chi- } \\
\text { Square } \\
\text { Likelihood Ratio } \\
\text { Fisher's Exact } \\
\text { Test } \\
\text { Linear-by-Linear } \\
\text { Association } \\
\mathrm{N} \text { of Valid Cases }\end{array}$ & $\begin{array}{r}1.447^{\mathrm{a}} \\
1.477 \\
1.415 \\
.180^{\mathrm{b}} \\
28\end{array}$ & $\begin{array}{l}2 \\
2\end{array}$ & $\begin{array}{r}.485 \\
.478\end{array}$ & $\begin{array}{l}.537 \\
.537 \\
.602 \\
.790\end{array}$ & .439 & .194 \\
\hline
\end{tabular}

a. 4 cells $(66.7 \%)$ have expected count less than 5 . The minimum expected count is 2.36 .

b. The standardized statistic is -.424 .

Table 20a: Cross-tabulation of survey respondents' location and digital action campaign participation. 
Questions 4 and 18: Which region of the world do you live in? * Have you taken the Voices of Our Future training on WorldPulse.com?

\begin{tabular}{|c|c|c|c|}
\hline \multirow{2}{*}{$\begin{array}{l}\text { Which region of the world } \\
\text { do you live in? }\end{array}$} & \multicolumn{2}{|c|}{$\begin{array}{l}\text { Have you taken the Voices of Our } \\
\text { Future training on WorldPulse.com? }\end{array}$} & \multirow[b]{2}{*}{ Total } \\
\hline & Yes & No & \\
\hline North America & 1 & 6 & 7 \\
\hline Africa & 10 & 8 & 18 \\
\hline Other & 10 & 3 & 13 \\
\hline Total & 21 & 17 & 38 \\
\hline
\end{tabular}

Table 21: Cross-tabulation of survey respondents' location and Voices of Our Future training participation. 


\section{Chi-Square Tests}

\begin{tabular}{|c|c|c|c|c|c|c|}
\hline & Value & df & $\begin{array}{l}\text { Asymp. } \\
\text { Sig. (2- } \\
\text { sided) }\end{array}$ & $\begin{array}{c}\text { Exact Sig. } \\
\text { (2-sided) }\end{array}$ & $\begin{array}{c}\text { Exact Sig. } \\
\text { (1-sided) }\end{array}$ & $\begin{array}{c}\text { Point } \\
\text { Probability }\end{array}$ \\
\hline $\begin{array}{l}\text { Pearson Chi- } \\
\text { Square } \\
\text { Likelihood Ratio } \\
\text { Fisher's Exact } \\
\text { Test } \\
\text { Linear-by-Linear } \\
\text { Association } \\
\text { N of Valid Cases }\end{array}$ & $\begin{array}{r}7.222^{\mathrm{a}} \\
7.740 \\
6.945 \\
6.679^{\mathrm{b}} \\
38\end{array}$ & 2 & $\begin{array}{l}.027 \\
.021\end{array}$ & $\begin{array}{l}.028 \\
.028 \\
.036\end{array}$ & .008 & .006 \\
\hline
\end{tabular}

a. 2 cells $(33.3 \%)$ have expected count less than 5 . The minimum expected count is 3.13 .

b. The standardized statistic is -2.584 .

\section{Table 21a: Cross-tabulation of survey respondents' location and Voices of Our} Future training participation.

Tables 20, 20a, 21 and 21a show that there is no connection between the region of the world participants live in and the level of participation in both the digital action campaigns and voices of our future training program. If one is to go by the assumption and stereotype that living in urban rather than a rural area, or a developed rather than a developing country, affects the quality of technical apparatuses, this does not seem to appear to be the case in the responses to the survey data. The tables indicate that other factors beside location affect respondents' participation in the digital action campaigns and 'Voices of Our Future' training. 
Appendix 4

Questions 18 and 14: Have you taken the Voices of Our Future training on WorldPulse.com? * Have you participated in any of the Digital Action Campaigns on WorldPulse.com?

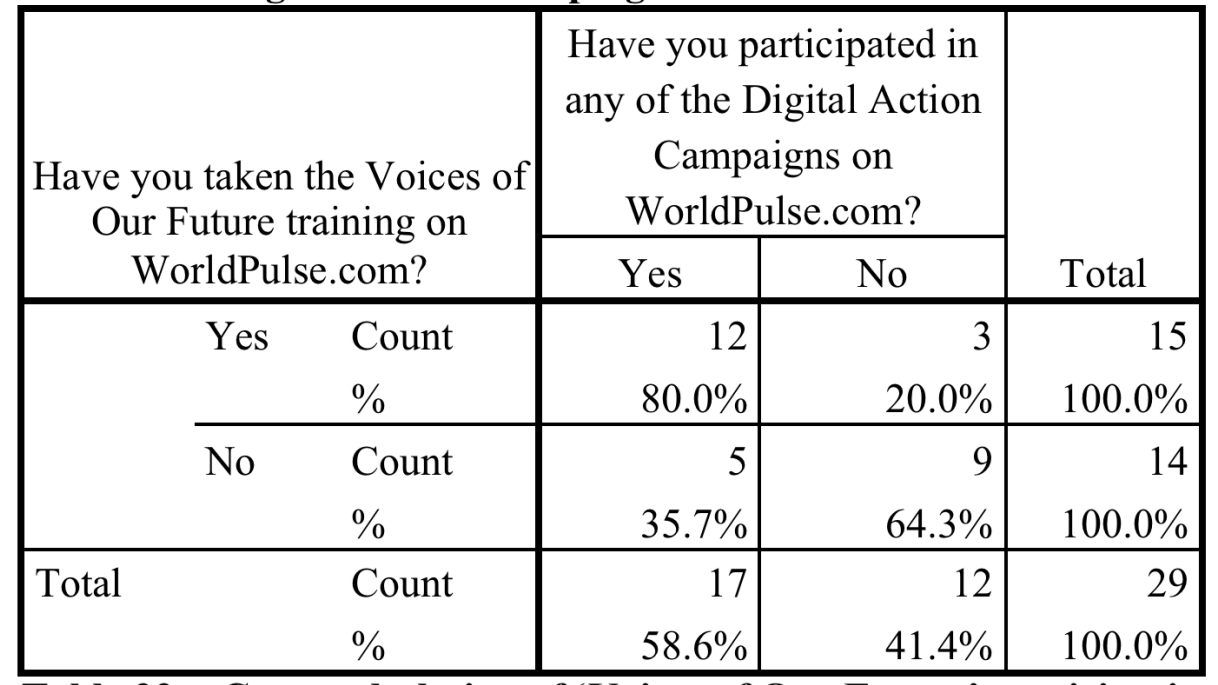

Table 22a: Cross-tabulation of 'Voices of Our Future' participation and digital action campaign participation 


\section{Chi-Square Tests}

\begin{tabular}{|c|c|c|c|c|c|}
\hline & Value & $\mathrm{df}$ & $\begin{array}{l}\text { Asymp. } \\
\text { Sig. (2- } \\
\text { sided) }\end{array}$ & $\begin{array}{l}\text { Exact } \\
\text { Sig. (2- } \\
\text { sided) } \\
\end{array}$ & $\begin{array}{l}\text { Exact } \\
\text { Sig. (1- } \\
\text { sided) } \\
\end{array}$ \\
\hline $\begin{array}{l}\text { Pearson Chi- } \\
\text { Square } \\
\text { Continuity } \\
\text { Correction }^{\mathrm{b}} \\
\text { Likelihood Ratio }^{-}\end{array}$ & $\begin{array}{l}5.855^{\mathrm{a}} \\
4.171 \\
6.075 \\
\end{array}$ & 1 & $\begin{array}{l}.016 \\
.041 \\
.014\end{array}$ & & \\
\hline $\begin{array}{l}\text { Fisher's Exact } \\
\text { Test } \\
\text { Linear-by-Linear } \\
\text { Association }\end{array}$ & 5.653 & 1 & .017 & .025 & .020 \\
\hline $\mathrm{N}$ of Valid Cases & 29 & & & & \\
\hline
\end{tabular}

a. 0 cells $(0.0 \%)$ have expected count less than 5 . The minimum expected count is 5.79 .

b. Computed only for a $2 \times 2$ table

\section{Table 22b: Cross-tabulation of 'Voices of Our Future' participation and digital action campaign participation}

Whether or not someone has participated in any of the digital action campaigns on WPc is associated with whether or not they have taken the voices of our future training $\left(\mathrm{X}^{2}(1)\right.$ $=4.171, \mathrm{p}=0.041$; applying Yate's continuity correction). $80.0 \%$ of those individuals

who have taken the training had participated in the digital action campaigns, compared to $35.7 \%$ of those individuals who had not taken the training. There is a very strong association between the DACs and VoF participation that suggests the communal relationship fostered during DACs potentially encourage participants to apply for and take the VoF training. 


\section{Appendix 5}

\section{T-test for questions 7 and 14}

\section{Group Statistics}

\begin{tabular}{|l|l|l|r|r|r|}
\hline & $\begin{array}{l}\text { Have you } \\
\text { participated in any } \\
\text { of the Digital } \\
\text { Action Campaigns } \\
\text { on } \\
\text { WorldPulse.com? }\end{array}$ & $\mathrm{N}$ & Mean & $\begin{array}{c}\text { Std. } \\
\text { Deviation }\end{array}$ & $\begin{array}{c}\text { Std. Error } \\
\text { Mean }\end{array}$ \\
\hline $\begin{array}{l}\text { How much do you } \\
\text { earn per year? }\end{array}$ & Yes & 13 & 21207.69 & $\begin{array}{r}20044.51 \\
1\end{array}$ & 5559.347 \\
& No & 10 & 35856.00 & $\begin{array}{r}56423.89 \\
0\end{array}$ & 17842.801 \\
\hline
\end{tabular}

Table 23a: Level of income and digital action campaign participation

Independent Samples Test

\begin{tabular}{|c|c|c|c|c|c|c|c|c|c|}
\hline \multirow{3}{*}{$\begin{array}{l}\text { How much do } \\
\text { you earn per } \\
\text { year? }\end{array}$} & \multicolumn{2}{|c|}{$\begin{array}{l}\text { Levene's } \\
\text { Test for } \\
\text { Equality of } \\
\text { Variances }\end{array}$} & \multicolumn{6}{|c|}{ t-test for Equality of Means } & \\
\hline & & & & & Sig. (2- & Mean & Std. Error & $\begin{array}{c}95 \% \\
\text { Confiden } \\
\text { ce } \\
\text { Interval } \\
\text { of the } \\
\text { Differenc } \\
\mathrm{e} \\
\end{array}$ & \\
\hline & $\mathrm{F}$ & Sig. & $\mathrm{t}$ & $\mathrm{df}$ & tailed) & Difference & Difference & Lower & Upper \\
\hline \begin{tabular}{|l} 
Equal \\
variances \\
assumed \\
\end{tabular} & 3.182 & .089 & -.872 & 21 & .393 & -14648.308 & 16793.386 & $\begin{array}{r}49572.06 \\
5 \\
\end{array}$ & $\begin{array}{r}20275 . \\
450\end{array}$ \\
\hline $\begin{array}{l}\text { Equal } \\
\text { variances } \\
\text { not } \\
\text { assumed }\end{array}$ & & & -.784 & 10.756 & .450 & -14648.308 & 18688.817 & $\begin{array}{r}55896.15 \\
5\end{array}$ & $\begin{array}{r}26599 . \\
539\end{array}$ \\
\hline
\end{tabular}

Table 23b: Level of income and digital action campaign participation

Tables $23 \mathrm{a}$ and $23 \mathrm{~b}$ show that the 13 survey respondents who indicated they had participated in the Digital Action Campaigns reported a mean income of US\$21,207.69. 


\section{Appendix 6}

\section{T-test for questions 7 and 18}

\section{Group Statistics}

\begin{tabular}{|l|l|r|r|r|c|}
\hline & $\begin{array}{l}\text { Have you taken the } \\
\text { Voices of Our } \\
\end{array}$ & & & & \\
& Future training on & & & Std. & Std. Error \\
& WorldPulse.com? & $\mathrm{N}$ & Mean & Deviation & Mean \\
\hline How much do you & Yes & 15 & 26220.00 & 46237.681 & 11938.518 \\
earn per year? & No & 15 & 24157.33 & 22579.976 & 5830.125 \\
\hline
\end{tabular}

Table 24a: Level of income and 'Voices of Our Future' participation

Independent Samples Test

\begin{tabular}{|c|c|c|c|c|c|c|c|c|c|}
\hline \multirow{3}{*}{$\begin{array}{l}\text { How much do } \\
\text { you earn per } \\
\text { year? }\end{array}$} & \multicolumn{2}{|c|}{$\begin{array}{l}\text { Levene's } \\
\text { Test for } \\
\text { Equality of } \\
\text { Variances }\end{array}$} & \multicolumn{7}{|c|}{ t-test for Equality of Means } \\
\hline & \multirow[b]{2}{*}{$\mathrm{F}$} & \multirow[b]{2}{*}{ Sig. } & \multirow[b]{2}{*}{$\mathrm{t}$} & \multirow[b]{2}{*}{ df } & \multirow{2}{*}{$\begin{array}{l}\text { Sig. (2- } \\
\text { tailed) }\end{array}$} & \multirow{2}{*}{$\begin{array}{c}\text { Mean } \\
\text { Difference }\end{array}$} & \multirow{2}{*}{$\begin{array}{l}\text { Std. Error } \\
\text { Difference }\end{array}$} & \multicolumn{2}{|c|}{$\begin{array}{c}95 \% \text { Confidence } \\
\text { Interval of the } \\
\text { Difference }\end{array}$} \\
\hline & & & & & & & & Lower & Upper \\
\hline $\begin{array}{l}\text { Equal } \\
\text { variances } \\
\text { assumed } \\
\text { Equal } \\
\text { variances not } \\
\text { assumed }\end{array}$ & .392 & .536 & .155 & 20.318 & .878 & 2062.667 & 13286.029 & $\begin{array}{r}- \\
25152 . \\
530 \\
- \\
25623 . \\
710\end{array}$ & $\begin{array}{r}29277.8 \\
63 \\
\\
29749.0 \\
44\end{array}$ \\
\hline
\end{tabular}

Table 24b: Level of income and 'Voices of Our Future' participation

Tables 24a and 24b indicate that there is a link between survey respondents' level of income and their participation in the DACs and VOF. More than half of income earners participated in the DACs, exactly half participated in the VOF. These are people who are otherwise engaged in the workforce and gainfully employed yet also making time to participate in these activities that are voluntary and require an amount of time commitment. 
Appendix 7

Questions 5 and 14: What is your highest level of education? * Have you participated in any of the Digital Action Campaigns on WorldPulse.com?

\begin{tabular}{|c|c|c|c|}
\hline \multirow{2}{*}{$\begin{array}{l}\text { What is your highest level of } \\
\text { education? }\end{array}$} & \multicolumn{2}{|c|}{$\begin{array}{c}\text { Have you participated in any } \\
\text { of the Digital Action } \\
\text { Campaigns on } \\
\text { WorldPulse.com? }\end{array}$} & \multirow[b]{2}{*}{ Total } \\
\hline & Yes & No & \\
\hline Bachelors & 7 & 3 & 10 \\
\hline Postgrad & 10 & 5 & 15 \\
\hline Other & 0 & 3 & 3 \\
\hline Total & 17 & 11 & 28 \\
\hline
\end{tabular}

Table 25a: Cross-tabulation of survey respondents' level of education and digital action campaign participation.

Chi-Square Tests

\begin{tabular}{|c|c|c|c|c|c|c|}
\hline & Value & $\mathrm{df}$ & $\begin{array}{c}\text { Asymp. Sig. } \\
\text { (2-sided) }\end{array}$ & $\begin{array}{c}\text { Exact Sig. } \\
\text { (2-sided) }\end{array}$ & $\begin{array}{c}\text { Exact Sig. } \\
\text { (1-sided) }\end{array}$ & $\begin{array}{c}\text { Point } \\
\text { Probability }\end{array}$ \\
\hline $\begin{array}{l}\text { Pearson Chi- } \\
\text { Square } \\
\text { Likelihood Ratio } \\
\text { Fisher's Exact } \\
\text { Test } \\
\text { Linear-by-Linear } \\
\text { Association } \\
\text { N of Valid Cases }\end{array}$ & $\begin{array}{r}5.221^{\mathrm{a}} \\
6.208 \\
4.589 \\
2.718^{\mathrm{b}} \\
28\end{array}$ & $\begin{array}{l}2 \\
2\end{array}$ & $\begin{array}{l}.074 \\
.045\end{array}$ & $\begin{array}{l}.098 \\
.098 \\
.112\end{array}$ & .088 & .064 \\
\hline
\end{tabular}

a. 3 cells $(50.0 \%)$ have expected count less than 5 . The minimum expected count is 1.18 .

b. The standardized statistic is 1.649 .

Table 25b: Cross-tabulation of survey respondents' level of education and digital action campaign participation.

Tables 25a and 25b: a test for independence was done using Fisher's Exact test because the cell count is small and we found that whether or not someone has participated in the $\mathrm{DAC}$ is independent of their level of education $(\mathrm{P}=0.112)$. However 10 of the 17 survey respondents have postgraduate educational qualification. 
Appendix 8

Questions 5 and 18: What is your highest level of education? *

Have you taken the Voices of Our Future training on

WorldPulse.com?

\begin{tabular}{|c|r|r|r|}
\hline \multirow{2}{*}{$\begin{array}{l}\text { What is your highest level of } \\
\text { education? }\end{array}$} & $\begin{array}{c}\text { Have you taken the Voices } \\
\text { of Our Future training on } \\
\text { WorldPulse.com? }\end{array}$ & \multirow{2}{*}{ Total } \\
\cline { 2 - 4 } & Yes & No & 14 \\
Bachelors & 9 & 5 & 21 \\
Postgrad & 13 & 8 & 3 \\
Other & 0 & 3 & 38 \\
\hline
\end{tabular}

Table 26: Cross-tabulation of survey respondents' level of education and participation in the 'Voices of Our Future' training.

Table 26 indicates that nine of the 22 survey respondents who took the 'Voices of Our Future' training have Bachelor's degrees while 13 have Postgraduate degrees. 
Appendix 9

Questions 2 and 9: What is your age bracket? * How many hours a week do you spend on WorldPulse.com?

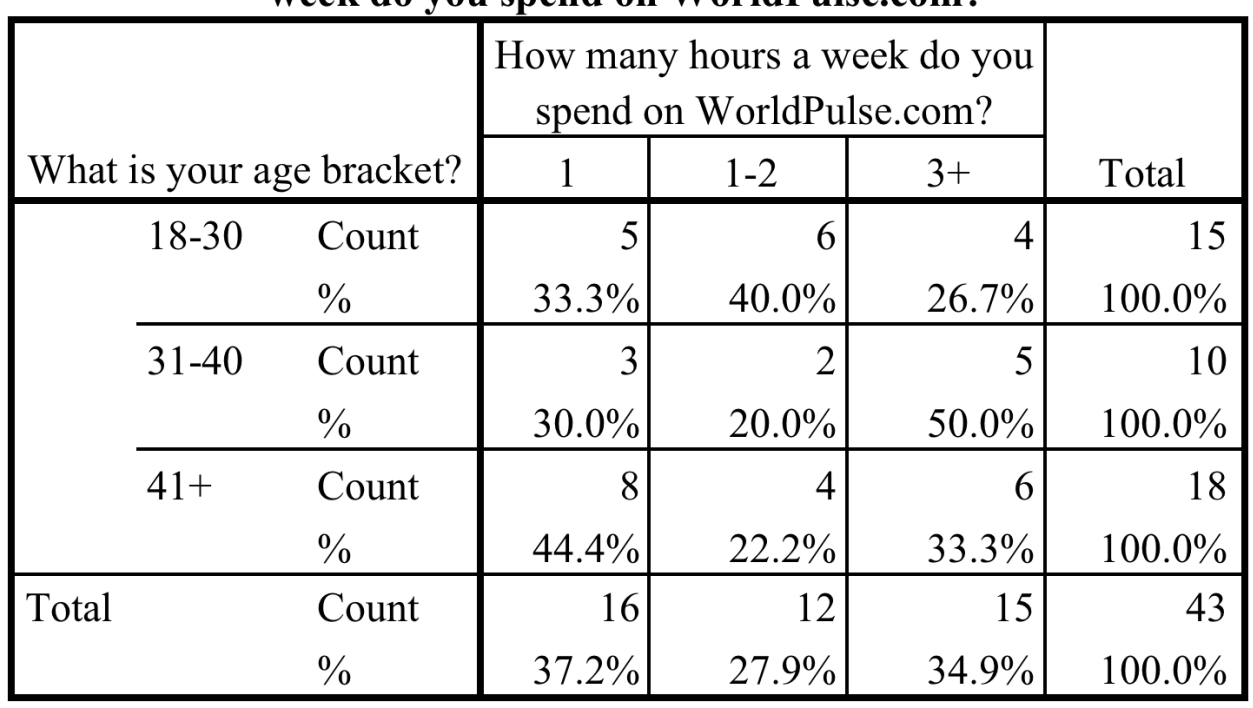

Table 27: Relationship between survey respondents' age and time spent on WorldPulse.com

Table 27 indicates, based on the cross-tabulation of WPc members' age and time spent on WPc and using Fisher's Exact Test $(\mathrm{p}=0.686)$ it was determined that the hours per week spent on the site is independent of the age of the individuals. Fisher's Exact Test was used because the cell counts are small. Of those aged between 18-30 six of them spend 12 hours per week on WPc; in the 31-40 age group five out of 10 spend $3+$ hours on WPc while in the 41 and above age group 8 out of 18 respondents spend up to 1 hour per week on WPc. 
Appendix 10

Questions 2 and 12: What is your age bracket? * Are you a member of any of the sub-committees on WorldPulse.com?

\begin{tabular}{|c|c|c|c|c|c|}
\hline & & & $\begin{array}{r}\text { Are you a mer } \\
\text { the sub-cor } \\
\text { WorldPu }\end{array}$ & $\begin{array}{l}\text { f any of } \\
\text { es on } \\
\text { ?? }\end{array}$ & \\
\hline What & your a & bracket? & Yes & No & Total \\
\hline & $18-30$ & Count & 11 & 3 & 14 \\
\hline & & $\%$ & $78.6 \%$ & $21.4 \%$ & $100.0 \%$ \\
\hline & $31-40$ & Count & 5 & 5 & 10 \\
\hline & & $\%$ & $50.0 \%$ & $50.0 \%$ & $100.0 \%$ \\
\hline & $41+$ & Count & 8 & 10 & 18 \\
\hline & & $\%$ & $44.4 \%$ & $55.6 \%$ & $100.0 \%$ \\
\hline Total & & Count & 24 & 18 & 42 \\
\hline & & $\%$ & $57.1 \%$ & $42.9 \%$ & $100.0 \%$ \\
\hline
\end{tabular}

Table 28a: Relationship between survey respondents' age and sub-committee membership 
Chi-Square Tests

\begin{tabular}{|l|r|r|r|}
\hline & Value & df & $\begin{array}{c}\text { Asymp. Sig. } \\
\text { (2-sided) }\end{array}$ \\
\hline Pearson Chi- & $4.019^{\mathrm{a}}$ & 2 & .134 \\
Square & 4.222 & 2 & .121 \\
Likelihood Ratio & & & .061 \\
Linear-by-Linear & 3.522 & 1 & \\
Association & 42 & & \\
N of Valid Cases & & & \\
\hline
\end{tabular}

a. 1 cells $(16.7 \%)$ have expected count less than 5 .

The minimum expected count is 4.29 .

\section{Table 28b: Relationship between survey respondents' age and sub-committee} membership

Tables $28 \mathrm{a}$ and $28 \mathrm{~b}$ indicate that whether or not someone is a member of any subcommittees on WPc is independent of the age of the individual $\left(X^{2}(2)=4.019, p=\right.$ 0.134). ( 2 is the df and we put it in brackets, $X^{2}$ denotes the Pearson Chi-Square statistic). 


\section{Appendix 11}

Questions 2 and 6: What is your age bracket? * Occupation

\begin{tabular}{|c|c|c|c|}
\hline \multirow{2}{*}{$\begin{array}{l}\text { What is your age } \\
\text { bracket? }\end{array}$} & \multicolumn{2}{|c|}{ Occupation } & \multirow[b]{2}{*}{ Total } \\
\hline & Paid & Unpaid & \\
\hline $18-30$ & 15 & 1 & 16 \\
\hline $31-40$ & 10 & 1 & 11 \\
\hline $41+$ & 16 & 3 & 19 \\
\hline Total & 41 & 5 & 46 \\
\hline
\end{tabular}

Table 29a: Cross-tabulation of nature of survey respondents' work and subcommittee membership.

\section{Chi-Square Tests}

\begin{tabular}{|c|c|c|c|c|c|c|}
\hline & Value & $\mathrm{df}$ & $\begin{array}{l}\text { Asymp. Sig. } \\
\text { (2-sided) }\end{array}$ & $\begin{array}{l}\text { Exact Sig. } \\
\text { (2-sided) }\end{array}$ & $\begin{array}{l}\text { Exact Sig. } \\
\text { (1-sided) }\end{array}$ & $\begin{array}{c}\text { Point } \\
\text { Probability }\end{array}$ \\
\hline Pearson Chi- & $.863^{\mathrm{a}}$ & 2 & .650 & .835 & & \\
\hline Square & & & & & & \\
\hline Likelihood Ratio & .870 & 2 & .647 & .835 & & \\
\hline Fisher's Exact & 875 & & & 835 & & \\
\hline Test & & & & & & \\
\hline $\begin{array}{l}\text { Linear-by-Linear } \\
\text { Association }\end{array}$ & $.813^{\mathrm{b}}$ & 1 & .367 & .434 & .269 & .145 \\
\hline $\mathrm{N}$ of Valid Cases & 46 & & & & & \\
\hline
\end{tabular}

a. 3 cells $(50.0 \%)$ have expected count less than 5 . The minimum expected count is 1.20 .

b. The standardized statistic is .902 .

Table 29b: Cross-tabulation of nature of survey respondents' work and subcommittee membership.

Tables $29 \mathrm{a}$ and $29 \mathrm{~b}$ indicate that a test for independence was conducted using Fisher's

Exact test, because the cell count is small. The tables show that whether or not someone

is in paid employment is independent of their age group $(\mathrm{P}=0.835)$.

The majority of the survey respondents across all the age groups are in paid employment

(41 out of 46 respondents). 


\section{Appendix 12}

Questions 2 and 14: What is your age bracket? * Have you participated in any of the Digital Action Campaigns on WorldPulse.com?

\begin{tabular}{|l|r|r|r|}
\hline \multirow{2}{*}{$\begin{array}{l}\text { What is your age } \\
\text { bracket? }\end{array}$} & \multicolumn{2}{|c|}{$\begin{array}{c}\text { Have you participated in any of the } \\
\text { Digital Action Campaigns on } \\
\text { WorldPulse.com? }\end{array}$} & \multirow{2}{*}{ Total } \\
\cline { 2 - 3 } & Yes & No & Tor \\
\cline { 2 - 3 } & 5 & 5 & 10 \\
$31-40$ & 3 & 4 & 7 \\
$41+$ & 9 & 3 & 12 \\
Total & 17 & 12 & 29 \\
\hline
\end{tabular}

Table 30a: Cross-tabulation of survey respondents' age and digital action campaign participation.

Chi-Square Tests

\begin{tabular}{|c|c|c|c|c|c|c|}
\hline & Value & $\mathrm{df}$ & $\begin{array}{l}\text { Asymp. Sig. } \\
\text { (2-sided) }\end{array}$ & $\begin{array}{c}\text { Exact Sig. } \\
\text { (2-sided) }\end{array}$ & $\begin{array}{c}\text { Exact Sig. } \\
\text { (1-sided) }\end{array}$ & $\begin{array}{c}\text { Point } \\
\text { Probability }\end{array}$ \\
\hline $\begin{array}{l}\text { Pearson Chi- } \\
\text { Square }\end{array}$ & $2.351^{\mathrm{a}}$ & 2 & .309 & .393 & & \\
\hline Likelihood Ratio & 2.416 & 2 & .299 & .393 & & \\
\hline $\begin{array}{l}\text { Fisher's Exact } \\
\text { Test }\end{array}$ & 2.364 & & & .355 & & \\
\hline $\begin{array}{l}\text { Linear-by-Linear } \\
\text { Association } \\
\mathrm{N} \text { of Valid Cases }\end{array}$ & $\begin{array}{r}1.456^{\mathrm{b}} \\
29\end{array}$ & 1 & .228 & .287 & .161 & .083 \\
\hline
\end{tabular}

a. 4 cells $(66.7 \%)$ have expected count less than 5 . The minimum expected count is 2.90 .

b. The standardized statistic is -1.207 .

Table 30b: Cross-tabulation of survey respondents' age and digital action campaign participation.

Tables 30a and 30b indicate that those 41 years old and above are more likely to participate in the digital action campaigns than any of the other age groups. 
Appendix 13

Questions 3 and 14: What is your marital status? * Have you participated in any of the Digital Action Campaigns on WorldPulse.com?

\begin{tabular}{|c|r|r|r|}
\hline \multirow{2}{*}{$\begin{array}{l}\text { What is your } \\
\text { marital status? }\end{array}$} & \multicolumn{2}{|c|}{$\begin{array}{l}\text { Have you participated in any of the Digital } \\
\text { Action Campaigns on WorldPulse.com? }\end{array}$} & \multirow{2}{*}{ Total } \\
\cline { 2 - 4 } Single & Yes & No & 16 \\
Married & 9 & 7 & 9 \\
Other & 7 & 2 & 4 \\
Total & 1 & 3 & 29 \\
\hline
\end{tabular}

Table 31a: Cross-tabulation of survey respondents' marital status and digital action campaign participation.

Chi-Square Tests

\begin{tabular}{|c|c|c|c|c|c|c|}
\hline & Value & $\mathrm{df}$ & $\begin{array}{l}\text { Asymp. } \\
\text { Sig. (2- } \\
\text { sided) }\end{array}$ & $\begin{array}{l}\text { Exact Sig. } \\
\text { (2-sided) }\end{array}$ & $\begin{array}{c}\text { Exact Sig. } \\
\text { (1-sided) }\end{array}$ & $\begin{array}{c}\text { Point } \\
\text { Probability }\end{array}$ \\
\hline $\begin{array}{l}\text { Pearson Chi- } \\
\text { Square } \\
\text { Likelihood Ratio } \\
\text { Fisher's Exact } \\
\text { Test } \\
\text { Linear-by-Linear } \\
\text { Association } \\
\mathrm{N} \text { of Valid Cases }\end{array}$ & $\begin{array}{r}3.263^{\mathrm{a}} \\
3.373 \\
3.069 \\
.247^{\mathrm{b}} \\
29\end{array}$ & $\begin{array}{l}2 \\
2\end{array}$ & $\begin{array}{l}.196 \\
.185\end{array}$ & $\begin{array}{l}.289 \\
.289 \\
.289\end{array}$ & .403 & .177 \\
\hline
\end{tabular}

a. 3 cells $(50.0 \%)$ have expected count less than 5 . The minimum expected count is 1.66.

b. The standardized statistic is .497 .

Table 31b: Cross-tabulation of survey respondents' marital status and digital action campaign participation.

Tables $31 \mathrm{a}$ and $31 \mathrm{~b}$ indicate that a test for independence was conducted using Fisher's

Exact test, because the cell count is small. The tables show that whether or not someone has 
participated in the DAC is independent of their marital status $(\mathrm{P}=0.289)$. Presumably this result could change if we assume that everyone who skipped the question did not participate in the digital action campaigns. Overall there are more single people than any of the married or other groups participating in the digital action campaigns. 
Appendix 14

Questions 9 and 12: How many hours a week do you spend on

WorldPulse.com? * Are you a member of any of the subcommittees on WorldPulse.com?

\begin{tabular}{|c|c|c|c|}
\hline \multirow{2}{*}{$\begin{array}{l}\text { How many hours a week } \\
\text { do you spend on } \\
\text { WorldPulse.com? }\end{array}$} & \multicolumn{2}{|c|}{$\begin{array}{l}\text { Are you a member of any of } \\
\text { the sub-committees on } \\
\text { WorldPulse.com? }\end{array}$} & \multirow[b]{2}{*}{ Total } \\
\hline & Yes & No & \\
\hline 1 & 6 & 9 & 15 \\
\hline $1-2$ & 6 & 6 & 12 \\
\hline $3+$ & 12 & 3 & 15 \\
\hline Total & 24 & 18 & 42 \\
\hline
\end{tabular}

Table 32 Cross-tabulation of the number of hours survey respondents spend on WorldPulse.com in comparison to their role as sub-committee members, which is voluntary work.

Table 32 indicates that 24 of the 47 survey respondents are sub-committee members on WPc. This 24 represents a significant $50 \%$ without accounting for the additional survey respondents who may not be sub-committee members but also make voluntary contribution through the publication of their 'Voices of Our Future' articles that are unremunerated. Out of these 24 people, 12 spend more than three hours per week on WPc. 
Appendix 15

\section{Correspondence}

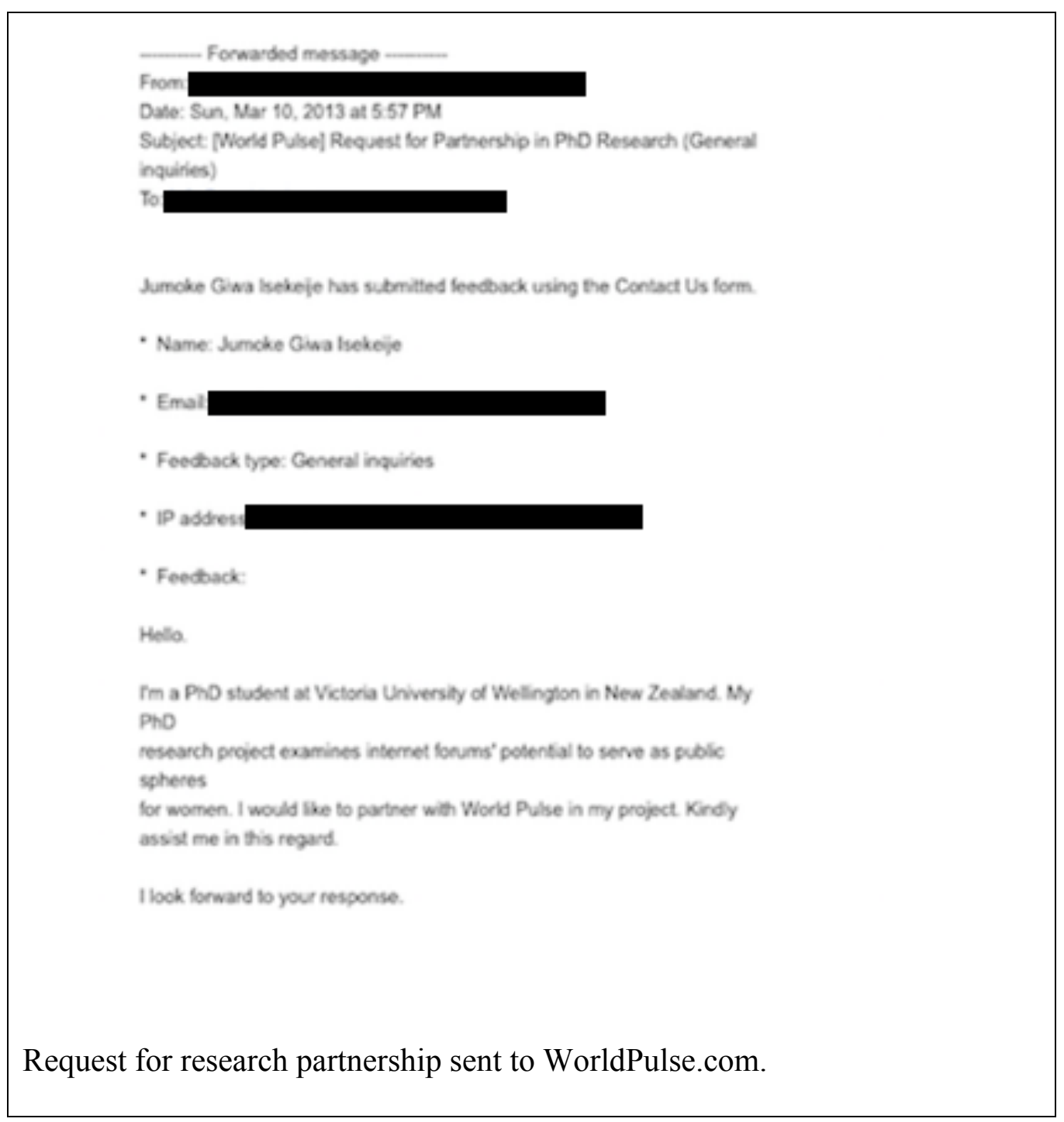




\section{SB}

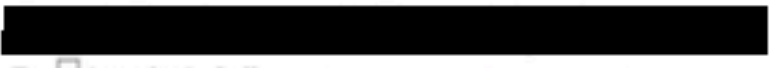

To: Drumoke isekeije; ₹

ล) MessageHeaderAnaly...

Thank you for following-up with me Jumoke. I appreciate your patience, as I was out on illness and vacation last week.

I think that your research project sounds very intriguing and important. I think that you would be able to conduct the research with very little needed from World Pulse staft, which is important given our limited capacity as a small staff.

You are able to connect personally with community members through comments or private messages for surveying, and can explain to the community that they can contact me if they have any questions or concerns. Do you think you would need more than this? I welcome any questions you might have for me at this point.

Kind regards,

Kind regards,

Response from WorldPulse.com to my request for research partnership. 
Appendix 16

\section{Ethics Approval}

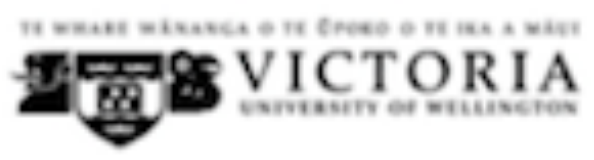

MEMORANDUM

Rime gatas in.

Vex gandas

tinel

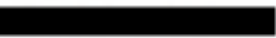

\begin{tabular}{|c|c|}
\hline to & Junche latkile \\
\hline copr & \\
\hline recu & 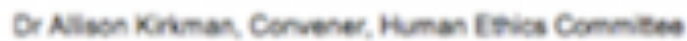 \\
\hline
\end{tabular}

\begin{tabular}{l|l}
\hline ONTE & 12 November 2013 \\
\hline PNots & 1 \\
\hline
\end{tabular}

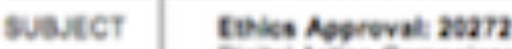

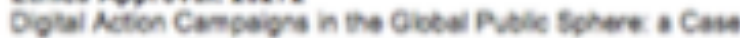
Study of Worvolse cem

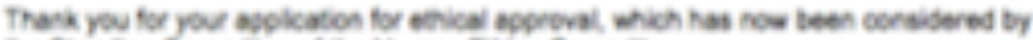
fre Sunsing Commitee of the Human Erics Commites.

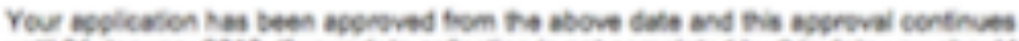

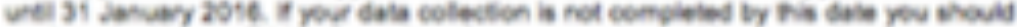

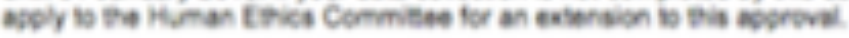

Best wiaves wh the resebrh

Alison Krimas

Human ERica Commites 
Appendix 17

\section{Web Survey Questions}

Digital Action Campaigns in the Global Public Sphere

Welcome!

Thank you for participating in this study. My name is Jumoke Giwa-Isekeije and I am a PhD Student in the School of English, Film, Theatre and Media Studies at Victoria University of Wellington in New Zealand. The aim of my doctoral research is to find out if women's participation in online forums can be construed as public sphere interactions.

Public sphere refers to a metaphorical arena/space for debating and deliberating on issues of common interest to people. Digital action campaigns are periodic and ongoing advocacy through which people express their opinions about topical or more established issues. These campaigns occur online but sometimes also have offline versions that are used to supplement the activities that have occurred online. The results of this web survey will help me understand why you participate in the digital action campaigns on WorldPulse.com. The survey is anonymous and should take no longer than 10 minutes to complete. By taking part in this survey, you will be making a valuable contribution to public sphere research, especially in relation to women's participation in public debates and advocacy. Please complete this survey latest by Friday, March 13, 2015.

Before you begin, please read the following statements:

- I understand that the data I provide is anonymous, and that any personal information collected is for statistical reasons only.

- I understand that survey data will be password protected until it is destroyed five years after the conclusion of the project.

- I understand that the results may be used in the researcher's $\mathrm{PhD}$ thesis, presentations, conference papers and journal articles.

- If I have any questions I can contact the researcher, Jumoke Giwa at jumoke.giwa@vuw.ac.nz or her primary supervisor, Tony Schirato at tony.schirato@vuw.ac.nz.

If you accept all of the statements above, please proceed to the next stage to begin.

Thank you for your time. 
Q1 What is your gender?

O Male (1)

O Female (2)

Q2 What is your age bracket?

O (1)

O $18-30(2)$

O $31-40(3)$

O $41-50(4)$

O $51-60(5)$

O $61-70(6)$

O $70>(7)$

Q3 What is your marital status

O Single (1)

O Married (2)

O Common law (3)

O Divorced (4)

Q4 Which country do you live in?

Q5 What is your highest level of education?

Q6 What is your occupation/profession?

Q7 How much do you earn per year?

Q8 Why did you join WorldPulse.com?

Q9 How many hours a week do you spend on WorldPulse.com?

O (1)

O 1-2 hours (2)

O 3-4 hours (3)

O 5-6 hours (4)

○ $7>(5)$ 
Q10 How did you hear about WorldPulse.com?

Through my friend (1)

Through a family member (2)

Through work or business association (3)

Through online search engine (4)

Other sources (please state) (5)

Q11 When did you join WorldPulse.com?

Q12 Are you a member of any of the sub-committees on WorldPulse.com?

$\bigcirc$ Yes; which ones? (1)

No; why not? (2)

Q13 Which of the following information would you like to know about other participants when engaging with them on WorldPulse.com?

$\square$ Race (1)

$\square$ Gender (2)

Age (3)

$\square$ Profession (4)

$\square$ Level of education (5)

Digital Action Campaigns are periodic and ongoing advocacy through which people express their diverse opinions about topical or more established issues. These campaigns occur online but sometimes also have offline versions that are used to supplement the activities that have occurred online. WorldPulse.com ran Ending Violence Against Women, Girls Transform the World, and Women Weave the Web digital action campaigns in 2012, 2013 and 2014. If you have participated in any of these digital action campaigns on WorldPulse.com, please answer the following questions. If you have not participated in the digital action campaigns, please go to the next page by clicking the arrows below on the right.

Q14 Have you participated in any of the Digital Action Campaigns on WorldPulse.com?

Y Yes; which ones? (1)

No; why not? (2)

Q15 Why did you participate in the Digital Action Campaigns on WorldPulse.com? 
Q16 Would you like to be contacted for a follow-up interview to share your experience participating in the Digital Action Campaigns on WorldPulse.com?

O Yes; please provide your email address, phone number or Skype ID below (1)

\section{O No (2)}

Q17 Have you ever applied for the Voices of Our Future training on WorldPulse.com?

O Yes (1)

O No (2)

Q18 Have you taken the Voices of Our Future training on WorldPulse.com?

O Yes; which year? (1)

O No; why not? (2)

Q19 If you took the Voices of Our Future training, have you had any of your stories published in any media since you took the training?

O Yes; which media (1)

No (2)

Q20 Would you like to be contacted for a follow-up interview concerning your experience participating in the Voices of Our Future training program on WorldPulse.com?

Y Yes; please provide your email, phone number of Skype ID below (1)

O No (2) 
Appendix 18

Interview Questions - WorldPulse.com members and World Pulse staff

Research Project: Digital Action Campaigns in the Global Public Sphere

Phase III: Unstructured Interviews

Groups I and II: WorldPulse.com community members (survey respondents who participated in the Digital Actions Campaigns and Voices of Our Future training)

\section{Introduction email:}

Dear WorldPulse.com community member:

My name is Jumoke Giwa-Isekeije and I am a PhD Student at Victoria University of Wellington in New Zealand. Thank you for participating in the online survey for my research project. I also thank you for supplying your email address and indicating your willingness to participate in the interviews. This is my formal invitation to you to begin the interviews, which will further assist me in collecting the data needed for completing the research that aims to find out if women's participation in online forums can be construed as public sphere interactions.

These interviews are unstructured and they will be conducted through email exchanges between us over the next five to six weeks. The interviews can finish earlier than this of course. Once I receive your responses to each email I will reply with the additional questions for you to answer.

The primary purpose of the interviews is to collect additional data as follow-up to the answers you gave in the web survey you completed for this project. You may decline to answer any of the questions asked in the emails I send to you. You may also ask to completely discontinue the interviews if you feel uncomfortable with any of the questions.

Thank you once again for your contribution to this research. As soon as I receive your reply I will send you the questions to begin the interviews. Kindly reply this email latest by [date]. You are welcome to reply even earlier than this.

Thanks and best regards, 
Jumoke

Group III: WorldPulse.com community members (participants in the Digital Action Campaigns who provided their contact information at the end of their journal articles or comments online)

\section{Introduction email:}

Dear WorldPulse.com community member:

My name is Jumoke Giwa-Isekeije and I am a PhD Student at Victoria University of Wellington in New Zealand. I am also a community member on WorldPulse.com. I have been reviewing some of the digital action campaign submissions as part of my research project. Thank you for participating in the digital action campaigns. I also recently conducted a web survey of the WorldPulse.com community members.

I would like to invite you to participate in some interviews I'm conducting as part of my research project. The primary purpose of my research is to find out if women's participation in online forums can be construed as public sphere interactions. If you would like to participate in these interviews please consider this my formal invitation to you to begin the interviews, which will further assist me in collecting the additional data needed for completing the research.

These interviews are unstructured and they will be conducted through email exchanges between us over the next five to six weeks. The interviews can finish earlier than this of course. Once I receive your responses to each email I will reply with the additional questions for you to answer.

The primary purpose of the interviews is to collect additional data as follow-up to the answers I got through the web survey I conducted for this project. You may decline to answer any of the questions asked in the emails I send to you. You may also ask to completely discontinue the interviews if you feel uncomfortable with any of the questions.

Thank you once again for your contribution to this research. As soon as I receive your reply I will send you the questions to begin the interviews. Kindly reply this email latest by [date]. You are welcome to reply even earlier than this.

Thanks and best regards, 
Jumoke

Start of interview email, with ethics statement and first set of interview questions:

Thanks again for your willingness to participate in these interviews. Before you begin please read the following statements:

- I understand that the data I provide is anonymous, and that any personal information collected is for statistical reasons only.

- I understand that the interview data will be password protected until it is destroyed five years after the conclusion of the project.

- I understand that the results may be used in the researcher's $\mathrm{PhD}$ thesis, presentations, conference papers and journal articles.

- I can withdraw from the interview by declining to answer the questions and informing the researcher through reply email that I am no longer interested in continuing with the interviews.

- If I have any questions or concerns about this research I can contact the researcher, Jumoke Giwa-Isekeije at jumoke.giwa@vuw.ac.nz or her primary supervisor, Tony Schirato at tony.schirato@vuw.ac.nz.

If you accept all the statements above, please proceed to answer the first set of questions below.

For these questions I would like you to reflect on the tools you use for accessing the Internet as well as your experiences of accessing WorldPulse.com. Please write your answers directly below each question.

\section{Section I: Technology and Autonomy}

- Please state your name and country of residence.

- What kind of device do you use to access the Internet; for example desktop, laptop, mobile phone, or tablet?

- Does this device belong to you or do you share it with other people? 
- How does your experience of this device affect your use of the Internet generally and specifically your use of WorldPulse.com?

- From where do you access WorldPulse.com; for example are you mostly at home, work, school, with friends, family members, at a community centre or library when you access the website?

- How does your experience of where you are when you access WorldPulse.com affect your interactions on the website?

Kindly reply this email latest by [date]. You are welcome to reply even earlier than this.

Thank you for your time.

Follow-up questions to Section I:

- Developed as responses came in.

For these questions I would like you to reflect on what you are able to or unable to do on WorldPulse.com because of your skills in using the Internet, including the kind of language used and any other support you need to complete the tasks on the websites. Please write your answers directly below each question.

\section{Section II: Skill and Social Support}

- What kind of satisfaction do you get from being able to access the Internet and specifically WorldPulse.com to participate in the digital action campaigns as well as the Voices of Our Future training?

- What else do you do on WorldPulse.com apart from participating in the digital action campaigns and the Voices of Our Future training?

- How do the languages for communicating on WorldPulse.com affect your use of the website?

- If you experience any difficulty in using WorldPulse.com who do you ask for assistance and support? 
- What kinds of assistance and support do they give you so you can use the website?

- How do the assistance and support encourage you to continue to use WorldPulse.com?

Kindly reply this email latest by [date]. You are welcome to reply even earlier than this.

Follow-up questions to Section II:

- Developed as responses came in.

For these questions I would like you to reflect specifically on the Digital Action Campaigns you participated in on WorldPulse.com. Please write your answers directly below each question.

\section{Section III: Digital Action Campaigns}

- How would you describe your experience participating in the digital action campaigns on WorldPulse.com?

- Which specific digital action campaign(s) have you participated in?

- How do discussions develop during the digital action campaigns?

- Have you received information about the outcome of the digital action campaigns that you participated in?

- How has the digital action campaigns affected you or the work you do in your immediate community?

- What would you say are the advantages and disadvantages of online activism such as the digital action campaigns on WorldPulse.com?

- What are some of the cultural or language differences you have noticed between yourself and other participants when discussing about the issues in the digital action campaigns on WorldPulse.com? 
- To what extent do the discussions and interactions you have during the digital action campaigns help provide practical help and resolution to the issues you discuss during the campaigns?

Kindly reply this email latest by [date]. You are welcome to reply even earlier than this.

Follow-up questions to Section III:

- During the digital action campaigns, when there are differences of opinion between you and other participants, how do you handle these differences of opinion?

- Would you say that your opinions were represented in the outcome of the digital action campaigns?

- Do you find the issues on WorldPulse.com as relevant to issues in your local community?

- Would you consider yourself a part of a global community through your involvement in WorldPulse.com?

- To what extent do the online discussions and interactions on WorldPulse.com inflect, influence or direct your cultural and political activities offline?

- What kind of additional work do you think the WorldPulse.com platform should be doing?

For these questions I would like you to reflect specifically on the Voices of Our Future training that you participated in on WorldPulse.com. Please write your answers directly below each question.

\section{Section IV: Voices of Our Future training}

- How would you describe your experience participating in the Voices of Our Future training?

- Which year did you participate in the Voices of Our Future training? 
- What was your role in the Voices of Our Future training in the year that you participated?

- Since you took the Voices of Our Future training, how many articles have you written?

- How many of the articles you wrote have been published?

- How many radio or television interviews have you given?

- How many articles have you written specifically for publication on WorldPulse.com?

- Were you paid for the articles you wrote for publication on WorldPulse.com?

- In what ways have you used the training you received in the Voices of Our Future program in your immediate community?

Kindly reply this email latest by [date]. You are welcome to reply even earlier than this.

Follow-up questions to Section IV:

- What would you identify as the advantages and disadvantages of citizen journalism?

- To what extent do the Voices of Our Future training inflect or influence your cultural and political activities after the training?

- How would you describe your media literacy prior to participating in the Voices of Our Future training?

- How do your offline identities (based on your race, class, gender, education) affect your participation in the Voices of Our Future training?

- During the Voices of Our Future training, when there is a difference of opinion between you and your mentor, other trainees or World Pulse staff (perhaps because of culture or language) how do you handle these differences of opinion? 
- What would you identify as the advantages and disadvantages of receiving such citizen journalism training online?

- To what extent do the discussions and interactions you have during the citizen journalism training help provide practical help and advise for you to continue writing as a citizen journalist?

- Can you identify any cultural similarities or differences between you and WorldPulse.com staff or the citizen journalism training mentors?

- Finally those were the final set of questions. Thank you once again for participating in these interviews. Do you have anything you would like to clarify or any additional information you would like to add?

Kindly reply this email latest by [date]. You are welcome to reply even earlier than this.

Demographic Information and Reflexivity:

- How would you describe your gender?

- What is your age bracket?

- Have you participated in the Voices of Our Future training program on WorldPulse.com?

- In what ways do the discussions you have on WorldPulse.com affect the opinions you hold about the issues discussed?

- How do you approach difference in opinion with other participants when engaging on WorldPulse.com?

- Would you change your opinion on a specific issue based on another participant's position on the issue discussed on WorldPulse.com?

- What policy changes have you encountered in your country that you can connect to any of the activities you engage in on WorldPulse.com?

- How would you describe your experience participating on WorldPulse.com? 
- How has your participation on WorldPulse.com affected the work you do in your immediate community?

- What are some of the cultural or language differences you have noticed between yourself and other participants when discussing issues on WorldPulse.com?

- To what extent do the discussions and interactions you have on WorldPulse.com help provide practical help and resolution to the issues you identify in your community?

\section{Group IV: WorldPulse.com staff}

\section{Introduction email:}

Dear [World Pulse staff]:

I trust you're all doing well and hope you're enjoying the summer.

I'm following up with you about your offer of assistance with my research project. My name is Jumoke Giwa-Isekeije and I am a PhD Student at Victoria University of Wellington in New Zealand. Thank you again for your offer to assist in collecting data for my research project. I have now completed my assessment of the digital action campaign submissions and the data I collected from the web survey conducted earlier this year. I am currently in the process of conducting interviews to gather additional data for completing this research.

This is my formal invitation to you to begin the interviews, which will further assist me in collecting the data needed for completing the research to find out if women's participation in online forums can be construed as public sphere interactions. I recognize that different staff members have responsibility for different sections of the WorldPulse.com community. My interview questions are about the individual staff member's profile, WorldPulse.com profile, the Digital Action Campaigns and the Voices of Our Future training program. I would appreciate if you can please confirm the staff members that are best positioned to answer these questions so I can forward the questions to them directly.

These interviews are unstructured and they will be conducted through email exchanges between us. The primary purpose of these interviews is to collect additional data as followup to the data I have from assessing the digital action campaigns on WorldPulse.com, the data from the online web survey and the email interviews I'm conducting with WorldPulse.com community members. You may decline to answer any of the questions asked in these interviews. You may also ask to completely discontinue the interviews if you feel uncomfortable with any of the questions. 
Thank you once again for your contribution to this research. As soon as I receive your reply I will send you the questions to begin the interviews. Kindly reply this email latest by [date]. You are welcome to reply even earlier than this.

Thanks and best regards,

Jumoke

\section{Start of interview email, with ethics statement and first set of interview questions:}

Before you begin please read the following statements:

- I understand that the data I provide is anonymous, and that any personal information collected is for statistical reasons only.

- I understand that the interview data will be password protected until it is destroyed five years after the conclusion of the project.

- I understand that the results may be used in the researcher's $\mathrm{PhD}$ thesis, presentations, conference papers and journal articles.

- I can withdraw from the interview by declining to answer the questions and informing the researcher through reply email that I am no longer interested in continuing with the interviews.

- If I have any questions or concerns about this research I can contact the researcher, Jumoke Giwa-Isekeije at jumoke.giwa@vuw.ac.nz or her primary supervisor, Tony Schirato at tony.schirato@vuw.ac.nz.

If you accept all the statements above, please proceed to answer the first set of questions below.

Thank you for your time.

For these questions I would like you to reflect on yourself as a WorldPulse.com community member and staff. Please write your answers directly below each question.

\section{Section I: Staff profile}


- Please state your name, when you joined WorldPulse.com as a staff member, and your role on the team. If you were a participant on the WorldPulse.com website before you were employed, what did you do as a community member before becoming a paid staff?

- What kinds of cultural literacy or sensitivity training did you possess before or had to acquire after becoming a staff member of WorldPulse.com?

- How are the cultural similarities or differences between you and the participants on WorldPulse.com negotiated? Are they an advantage or a disadvantage?

Kindly reply this email latest by [date]. You are welcome to reply even earlier than this.

Follow-up questions to Section I:

- Developed as responses came in.

For these questions I would like you to reflect on WorldPulse.com as a public platform. Please write your answers directly below each question.

\section{Section II: WorldPulse.com profile}

- How would you characterize the communication practices on WorldPulse.com?

- To what extent are the communication practices on the site regulated, overseen or moderated?

- How do you address and resolve differences between the site participants on one hand and between the participants and the WorldPulse.com staff on the other, that arise perhaps because of class, race, gender, age, profession or level of education?

- What are the site's procedures and protocols for beginning and negotiating discussions and differences of opinion?

- How many members from how many countries altogether do you have on the WorldPulse.com website? How many of these members are active monthly, yearly? 
- One of the tasks of Volunteer Listeners (commentators) was to also rate the journal entries based on the criteria the WorldPulse.com management supplied to the volunteers. I was a Volunteer Listener for the Women Weave the Web campaign in 2014. Can I discuss the tasks I completed as a Volunteer Listener in this research project?

- Prior to the re-launch of the WorldPulse.com website in 2015, there was a record that there were over 60,000 registered members representing 96 countries in the WorldPulse.com community as of 2014. What is the number of registered participants as of today? How many of these registered participants were active in 2012, 2013 and 2014?

- What kind of additional work do you think the WorldPulse.com platform should be doing?

Kindly reply this email latest by [date]. You are welcome to reply even earlier than this.

Follow-up questions to Section II:

- Developed as responses came in.

For these questions I would like you to reflect on the Digital Action Campaigns that you run on WorldPulse.com. Please write your answers directly below each question.

\section{Section III: Digital Action Campaigns}

- You run Digital Action Campaigns on the WorldPulse.com website. What would you say are the advantages and disadvantages of online activism such as these campaigns?

- How do you determine which issues to address and promote for the Digital Action Campaigns?

- Why did WorldPulse.com decide to run the following digital action campaigns and focus on the issues they address: a) Ending Gender-Based Violence 2012, b) Girls Transform The World 2013, and c) Women Weave the Web 2014? 
- You produce reports to indicate the outcome of digital action campaigns after the campaigns have closed. Please provide the reports for the following three campaigns: a) Ending Gender-Based Violence 2012, b) Girls Transform The World 2013, and c) Women Weave the Web 2014. Please add these reports as attachments to the reply email.

- The summary in the introduction to the Ending Gender-Based Violence 2012 campaign indicates that a total of 153 WorldPulse.com community members contributed to the 618 journal entries. Do these 153 include participants who made comments or is it just the individual journal entries that were counted?

- The summary for the Girls Transform The World 2013 campaign indicates that there were "more than 350 submissions from more than 60 countries". Do these 350 submissions and 60 countries include the comments and location of those commenting on the individual journal entries?

- The summary for the Women Weave the Web 2014 campaign indicates that there were a total of " 590 submissions from nearly 70 countries around the globe". Do these 590 submissions and 70 countries include the comments and location of those commenting on the individual journal entries?

Kindly reply this email latest by [date]. You are welcome to reply even earlier than this.

Follow-up questions to Section III:

- Developed as responses came in.

For these questions I would like you to reflect on the Voices of Our Future training program that you run on WorldPulse.com. Please write your answers directly below each question.

\section{Section IV: Voices of Our Future training program}

- You conduct the Voices of Our Future training - a citizen journalism training program - online. What would you say are the advantages and disadvantages of running such programs online? 
- The profiles of the participants of the Voices of Our Future citizen journalism training program in 2009, 2011, 2012 and 2013 are available on the WorldPulse.com website. Some of the respondents to the web survey I conducted mentioned they had applied to a 2010 edition of the training. Was there a Voices of Our Future training in 2010? If yes please provide some information about the 2010 training.

- You have a database of all the participants, mentors and editorial midwives of the Voices of Our Future training. I want to conduct interviews specifically with these groups of WorldPulse.com community members through email. I would like to send the interview questions to you for distribution to the participants. Please confirm the procedure for this and when I should send the interview questions.

Kindly reply this email latest by [date]. You are welcome to reply even earlier than this.

Follow-up questions to Section IV:

- Developed as responses came in.

- Finally those were the final set of questions. Thank you once again for participating in these interviews. Do you have anything you would like to clarify or any additional information you would like to add? 
Appendix 19

\section{Code Book}

\begin{tabular}{|c|c|}
\hline Code & Definition \\
\hline \multicolumn{2}{|c|}{$\begin{array}{l}\text { I. Habermas: Public Sphere - Habermas } 1974 \\
\text { Six criteria of the public sphere operationalized by Lincoln Dahlberg }\end{array}$} \\
\hline $\begin{array}{l}\text { 1. Autonomy (from state and } \\
\text { economic power) }\end{array}$ & $\begin{array}{l}\text { Discourse must be based on the concerns of } \\
\text { citizens as a public rather than driven by the } \\
\text { media of money and administrative power } \\
\text { that facilitate the operations of the market } \\
\text { and state. }\end{array}$ \\
\hline $\begin{array}{l}\text { 2. Exchange and critique (of } \\
\text { criticizable moral-practical } \\
\text { validity claims) }\end{array}$ & $\begin{array}{l}\text { Deliberation involves engaging in reciprocal } \\
\text { critique of normative positions that are } \\
\text { provided with reasons and thus are open to } \\
\text { critique and criticism rather than being } \\
\text { dogmatically asserted. }\end{array}$ \\
\hline $\begin{array}{l}\text { 3. Discursive inclusion and } \\
\text { equality }\end{array}$ & $\begin{array}{l}\text { Every participant affected by the validity } \\
\text { claims under consideration is equally entitled } \\
\text { to introduce and question any assertion } \\
\text { whatsoever. Inclusion can be limited by } \\
\text { inequalities from outside of discourse - by } \\
\text { formal or informal restrictions to access. It } \\
\text { can also be limited through people struggling } \\
\text { to get their voices heard, and when some } \\
\text { [what we can call hegemonic] discursive } \\
\text { positions dominate the discussion. }\end{array}$ \\
\hline 4. Reflexivity & $\begin{array}{l}\text { Participants critically examine their cultural } \\
\text { values, assumptions, and interests, as well as } \\
\text { the larger social context. }\end{array}$ \\
\hline 5. Ideal role taking & $\begin{array}{l}\text { Participants attempt to understand the } \\
\text { argument from the other's perspective. This } \\
\text { requires a commitment to an ongoing } \\
\text { dialogue with difference in which } \\
\text { interlocutors respectfully listen to each other. }\end{array}$ \\
\hline
\end{tabular}




\begin{tabular}{|c|c|}
\hline 6. Sincerity & $\begin{array}{l}\text { Each participant make a sincere effort to } \\
\text { make known all information - including } \\
\text { their true intentions, interests, needs, and } \\
\text { desires - as relevant to the particular } \\
\text { problem under consideration. }\end{array}$ \\
\hline \multicolumn{2}{|c|}{$\begin{array}{l}\text { II. DiMaggio \& Hargittai: Digital Inequality - DiMaggio \& Hargittai } 2001 \\
\text { Five broad forms of digital inequality }\end{array}$} \\
\hline \multicolumn{2}{|c|}{$\begin{array}{l}\text { 1. Inequality in technical apparatus. } \\
\text { Hardware and connections by which people access the web }\end{array}$} \\
\hline Digital divide & $\begin{array}{l}\text { Mention of challenges with access to (or use) } \\
\text { of new technologies }\end{array}$ \\
\hline \multicolumn{2}{|c|}{$\begin{array}{l}\text { 2. Inequality in autonomy of use. } \\
\text { Extent to which people exercise autonomy in their use of the web (e.g. do they } \\
\text { access it from work or home, is the use monitored or unmonitored, or must they } \\
\text { compete with other users for time on-line) }\end{array}$} \\
\hline $\begin{array}{l}\text { Digital inequality } \\
\text { (individual/groups) }\end{array}$ & $\begin{array}{l}\text { Indication of inequality among participants } \\
\text { on the website; indicators include inadequate } \\
\text { skill and knowledge about the issues being } \\
\text { discussed }\end{array}$ \\
\hline $\begin{array}{l}\text { Digital inequality } \\
\text { (institutional) }\end{array}$ & $\begin{array}{l}\text { Inequality due to interactions among firms' } \\
\text { strategic choices, consumers' responses, and } \\
\text { government policies. Indicators include } \\
\text { reference to inadequacies in internet } \\
\text { technology access and use by WP } \\
\text { management. }\end{array}$ \\
\hline Digital literacy & $\begin{array}{l}\text { Evidence or indication of pre-existing digital } \\
\text { competence. }\end{array}$ \\
\hline \multicolumn{2}{|c|}{$\begin{array}{l}\text { 3. Inequality in skill. } \\
\text { Skill that people bring to their use of the internet, including language } \\
\text { proficiency and knowledge of the topic being discussed }\end{array}$} \\
\hline Capacity building - individual & $\begin{array}{l}\text { Building community. Enhancing } \\
\text { social/career status. Helping family and } \\
\text { friends. Indicators include reference and } \\
\text { evidence that a participant uses his/her skill } \\
\text { and knowledge to assist others or gains }\end{array}$ \\
\hline
\end{tabular}




\begin{tabular}{|l|l|}
\hline \multicolumn{1}{|l|}{} & $\begin{array}{l}\text { additional skill and knowledge through } \\
\text { his/her interactions on the website. }\end{array}$ \\
\hline Team based capacity building & $\begin{array}{l}\text { Community/group of participants growing as } \\
\text { a team based on their interaction on/off the } \\
\text { site or based on one participant's interaction } \\
\text { leading to off-line engagements (e.g. Maman } \\
\text { Shujaa Centre, Star of Hope Transformation } \\
\text { Centre, conference attendance and } \\
\text { presentations). }\end{array}$ \\
\hline Capacity building - institutional & $\begin{array}{l}\text { Building the capacity of institutions like } \\
\text { police, school, government agencies, and } \\
\text { community agencies. }\end{array}$ \\
\hline $\begin{array}{l}\text { 4. Inequality in the availability of social support. } \\
\text { Social support that internet users can draw on }\end{array}$ \\
\hline Digital inclusion & $\begin{array}{l}\text { Attempts by co-participants to be inclusive, } \\
\text { including offering to assist with skill } \\
\text { acquisition and knowledge about issues. } \\
\text { Linked to digital inequality } \\
\text { (individual/groups). }\end{array}$ \\
\hline $\begin{array}{l}\text { Support } \\
\text { individual or group } \\
\text { institutional }\end{array}$ & $\begin{array}{l}\text { Indications of spiritual and inspirational } \\
\text { support in the form of prayers or offers of } \\
\text { prayers and other spiritual assistance. }\end{array}$ \\
\hline $\begin{array}{l}\text { 5. Variation in use. } \\
\text { Purposes for which people use the technology. }\end{array}$ \\
\hline Digital affordances & $\begin{array}{l}\text { Mention or discussion of what access to the } \\
\text { internet or ability to use digital tools enable } \\
\text { people to do. }\end{array}$ \\
\hline $\begin{array}{l}\text { Indications of ability to complete certain } \\
\text { tasks due to knowledge of digital tools or } \\
\text { access to digital tools. }\end{array}$ \\
\hline $\begin{array}{l}\text { Indications of ability to complete certain } \\
\text { tasks due to knowledge of digital tools or } \\
\text { access to digital tools facilitated by } \\
\text { institutions. }\end{array}$ \\
\hline
\end{tabular}




\begin{tabular}{|c|c|}
\hline \multicolumn{2}{|c|}{$\begin{array}{l}\text { III. Civic Engagement: Embedded e-Activists - Giwa } 2015 \\
\text { Inductive typology for assessing civic engagement in online/offline } \\
\text { communities }\end{array}$} \\
\hline $\begin{array}{l}\text { 1. Demographics } \\
\text { - Age } \\
\text { - Gender } \\
\text { - Education } \\
\text { - Work/Business } \\
\text { - Race/Ethnicity } \\
\text { - Location }\end{array}$ & $\begin{array}{l}\text { Participants supply demographic information } \\
\text { in the posts submitted during the campaigns, } \\
\text { in the web survey or in response to interview } \\
\text { questions. }\end{array}$ \\
\hline 2. Issues & $\begin{array}{l}\text { Deliberating on political, social and cultural } \\
\text { issues. Indication of which of these issues } \\
\text { are on the agenda and which additional ones } \\
\text { have been introduced or raised/discussed by } \\
\text { the participants. }\end{array}$ \\
\hline 3a. Measures & $\begin{array}{l}\text { Instruments that exist or are being developed } \\
\text { to aid in addressing the issues and solving } \\
\text { the problems. }\end{array}$ \\
\hline 3b. Measurable action & $\begin{array}{l}\text { Changes in policy and mode of governance } \\
\text { in members' micro communities and larger } \\
\text { macro communities. Indicators include } \\
\text { participants' account of such changes } \\
\text { occurring in their communities. }\end{array}$ \\
\hline 4. Actions & $\begin{array}{l}\text { Steps taken by individuals/organizations to } \\
\text { make use of the measures that are available } \\
\text { for addressing the issues and solving the } \\
\text { problems. }\end{array}$ \\
\hline 5. Outcomes & $\begin{array}{l}\text { Report or mention of follow up or follow } \\
\text { through after issues have been identified and } \\
\text { addressed. }\end{array}$ \\
\hline 6. Expectations & $\begin{array}{l}\text { Measures that community members would } \\
\text { like to see put in place to alleviate issues of } \\
\text { inequality and ensure that people have access } \\
\text { to the tools they need to live a better life. }\end{array}$ \\
\hline 7. Recommendations & $\begin{array}{l}\text { Community members' suggestions on how to } \\
\text { address the issues that are being discussed. }\end{array}$ \\
\hline
\end{tabular}




\begin{tabular}{|l|l|}
\hline & \\
\hline 8. Solutions & $\begin{array}{l}\text { Indications that some identified issues have } \\
\text { been addressed/solved. Linked to Issues and } \\
\text { Recommendations. }\end{array}$ \\
\hline 9. Repercussions & $\begin{array}{l}\text { Punishment for going against norms or } \\
\text { expectations of institutions: family, school, } \\
\text { law, religion, society, government. Linked to } \\
\text { Issues, Recommendations, Solutions. }\end{array}$ \\
\hline 10. Volunteer(ism) & $\begin{array}{l}\text { Indication of people doing work without } \\
\text { being paid for it. This could be online on the } \\
\text { WPc platform or offline in local/immediate } \\
\text { or global communities. }\end{array}$ \\
\hline 11. Authentication & $\begin{array}{l}\text { Attempts to verify authenticity, status, } \\
\text { efficacy and credibility of participants' } \\
\text { demographic information and contributions } \\
\text { on the platform. }\end{array}$ \\
\hline 12. Value & $\begin{array}{l}\text { Expression of value the participants place on } \\
\text { their contributions to debates/deliberations } \\
\text { on WPc, in relation to economic and socio- } \\
\text { political issues in wider global communities. }\end{array}$ \\
\hline 13. Collective voice & $\begin{array}{l}\text { Participants see their } \\
\text { contributions/deliberations as representative } \\
\text { of a collective voice for women. }\end{array}$ \\
\hline
\end{tabular}

